\title{
The VE-cadherin/AmotL2 mechanosensory pathway suppresses aortic inflammation and the formation of abdominal aortic aneurysms
}

\section{Yuanyuan Zhang}

Karolinska Institute

\section{Evelyn Hutterer}

Karolinska Institute

Sara Hultin

Karolinska Institute

Otto Bergman

Karolinska Institute

\section{Maria Forteza}

Karolinska Institute

\section{Zorana Andonovic}

Karolinska Institute

\section{Daniel Ketelhuth}

Karolinska University Hospital, Stockholm, Sweden

Joy Roy

Karolinska Institute

Per Eriksson

Karolinska Institute

Lars Holmgren ( $\nabla$ Lars.Holmgren@ki.se )

Karolinska Institute

\section{Article}

Keywords: arterial endothelial cells (ECs), vascular disease, abdominal aortic aneurysms

Posted Date: June 15th, 2021

DOI: https://doi.org/10.21203/rs.3.rs-600069/v1

License: (c) (1) This work is licensed under a Creative Commons Attribution 4.0 International License.

Read Full License 


\section{The VE-cadherin/AmotL2 mechanosensory pathway suppresses aortic inflammation and the formation of abdominal aortic aneurysms}

Yuanyuan Zhang ${ }^{1}$, Evelyn Hutterer ${ }^{1}$, Sara Hultin ${ }^{1}$, Otto Bergman ${ }^{2}$, Maria J. Forteza ${ }^{2}$, Zorana Andonovic ${ }^{1}$, Daniel F.J. Ketelhuth ${ }^{2,3}$, Joy Roy ${ }^{4}$, Per Eriksson ${ }^{2}$ and Lars Holmgren ${ }^{1 *}$.

${ }^{1}$ Department of Oncology-Pathology, BioClinicum, Karolinska Institutet, Stockholm, Sweden.

${ }^{2}$ Department of Medicine Solna, BioClinicum, Karolinska Institutet, Karolinska University Hospital, Stockholm, Sweden.

${ }^{3}$ Department of Cardiovascular and Renal Research, Institutet of Molecular Medicine, Univ. of Southern Denmark, Odense, Denmark

${ }^{4}$ Department of Molecular Medicine and Surgery, Karolinska Institutet, Karolinska University Hospital, Stockholm, Sweden.

*To whom correspondence should be addressed.

Lars Holmgren: Lars.Holmgren@,ki.se, Cell: +46734036794 


\begin{abstract}
Arterial endothelial cells (ECs) have the ability to respond to mechanical forces exerted by fluid shear stress. This response is of importance, as it is protective against vascular diseases such as atherosclerosis and aortic aneurysms. Mechanical forces are transmitted at the sites of adhesion to the basal membrane as well as cell-cell junctions where protein complexes connect to the cellular cytoskeleton to relay force into the cell. Here we present a novel protein complex that connects junctional VE-cadherin and radial actin filaments to the LINC complex in the nuclear membrane. We show that the scaffold protein AmotL2 is essential for the formation of radial actin filaments and the flow-induced alignment of aortic and arterial ECs. The deletion of endothelial AmotL2 alters nuclear shape as well as subcellular positioning. Molecular analysis shows that VE-cadherin is mechanically associated with the nuclear membrane via binding to AmotL2 and Actin. Furthermore, the deletion of AmotL2 in ECs provokes a pro-inflammatory response and abdominal aortic aneurysms (AAA) in the aorta of mice on a normal diet. Remarkably, transcriptome analysis of AAA samples from human patients revealed a negative correlation between AmotL2 expression and aneurysm diameters, as well as a positive correlation between AmotL2 and YAP expression. These findings provide a conceptual framework regarding how mechanotransduction in the junctions is coupled with vascular disease.
\end{abstract}




\section{Introduction}

The blood vessel wall is lined with a thin layer of vascular endothelial cells (ECs), which form a barrier between the blood and tissues. These cells differ in biochemical characteristics depending on their localization in arteries or veins, as well as on the organ in which they reside. The endothelium is continuously exposed to the shear stress exerted by the blood flow. Understanding how ECs respond to shear stress is of importance as it has implications for the development of vascular diseases. Indeed, since the 1870's it has been postulated that mechanical stress exerted on the blood vessel wall may be a trigger of atherosclerosis. Also, low wall shear stress has been associated with abdominal aortic aneurysm (AAA) rupture ${ }^{1}$. AAA is characterized by localized medial and adventitial inflammation and dilatation of the abdominal aorta and is prevalent in men over 65 with significant morbidity and mortality ${ }^{2}$. By contrast, areas of laminar flow appeared relatively protected against the development of the inflammatory disease ${ }^{3}$.

To explore how the mechanotransductive pathways mediate protection or activation of the vascular disease is of clear importance $e^{4-18}$. To date, several mechanosensory pathways have been identified that relay external mechanical forces to the endothelial lining ${ }^{19}$. In vitro, flow-induced endothelial alignment is dependent on the activation of GTPases and consequent actin reorganization. In vivo, it has been shown that endothelial junctional protein complexes including PECAM-1, VE-cadherin, and VEGFR2 play an important role in the adaptive response to shear stress ${ }^{18}$. However, it is still not clear how mechanical forces exerted by the blood flow are transmitted from the junctions via the cytoskeleton into the cell.

Studies of the (Angiomotin) Amot protein family may provide important insights into this aspect. This is a family of scaffold proteins that link membrane receptors to the actin cytoskeleton, polarity proteins, and are implicated in modulating the Hippo pathway ${ }^{20-26}$. 
We have recently shown that one of its members, AmotL2 (p100 isoform), is associated with the VE-cadherin complex in ECs and E-cadherin in epithelial cells ${ }^{25,27}$. Silencing of AmotL2 in zebrafish, mouse or cells in vitro results in a loss of radial actin filaments that run perpendicular to the outer cell membrane. These actin filaments mechanically connect cells via binding to junctional cadherins, and thereby transmit force. Conditional silencing of AmotL2 in the endothelial lineage of mice inhibits expansion of the aorta during the onset of circulation resulting in death in utero at embryonic day $10^{25}$. In this report, we have analyzed the role of AmotL2 in controlling junctional and cytoskeletal components during the alignment of arterial ECs exposed to laminar flow. Here we present a novel mechanical transduction pathway active in arteries that is protective against vascular inflammation as well as the formation of AAAs. 


\section{Results}

\section{Amotl2 is essential for arterial alignment.}

We analyzed the expression pattern of AmotL2 in mouse descending aorta (DA) and the inferior vena cava (IVC) as shown in Fig. 1a,b. ECs of the DA were typically elongated and aligned with the direction of blood circulation and contained radial actin filaments that were connected to the cellular junctions (Fig. 1c). AmotL2 localized to EC junctions as previously reported ${ }^{25}$. In contrast, ECs of the IVC exhibited a more rounded cellular shape with no or few detectable radial actin filaments as well as a significantly lower expression level of AmotL2. Box plots in Fig. 1d-f represents the statistically significant difference between the DA and IVC with regards to AmotL2 expression, cellular shape and the presence of radial actin filaments. In addition, we mapped AmotL2 expression in mouse retinal vasculature at different ages (Supplementary Fig. 1). AmotL2 was expressed at similar levels in arteries and veins of post-natal day 6 mice, whereas in mature mice (three months) Amot12 was primarily expressed in retinal arteries. Interestingly, AmotL2 was barely detectable in blood vessels of mice older than 15 months.

We have previously shown that AmotL2 is required for the formation of radial actin filaments both in epithelial and endothelial cells ${ }^{25,27}$. The preferential expression of AmotL2 in EC of the aorta raised the possibility that AmotL2 controlled arterial EC shape via the formation of radial actin fibers. To address this question, we used a genetic deletion approach to silence amotl2 gene expression specifically in the endothelial lineage as previously reported ${ }^{28}$. In this model system, amotl2 flox/flox mice were crossed with Cdh5(PAC) ${ }^{\text {CreERT2 }}$ transgenics as well as ROSA26-EYFP reporter mice ${ }^{25}$, from now on referred to as amotl $2^{e c / e c}$. This crossing enables efficient inducible conditional recombinase expression and subsequent amotl2 knockout in ECs (amotl2 $\left.2^{e c-l e c-}\right)$ after tamoxifen injections as well as quantification of recombination efficiency by YFP expression (Supplementary 
Fig. 2a,b). Adult mice (7-9 months old) were euthanized one month after tamoxifen injections, and aortae were dissected and analyzed by whole-mount immunostaining. Inactivation of AmotL2 in the DA resulted in the loss of radial actin filaments and altered

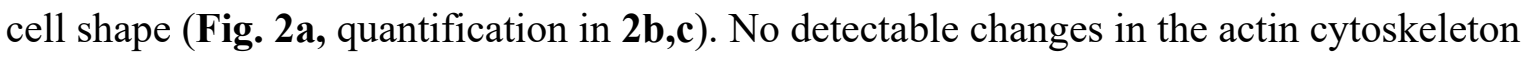
and cellular shape were observed in the endothelium of the IVC of amotl2e-/ec- mice. This observed phenotype change appeared to be arterial-specific as similar effects were observed in arterial, but not venous ECs of other organs such as the urinary bladder (Fig. 2d,

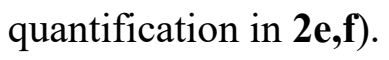

The nucleus is the largest organelle of the EC. As such it is exposed to the hemodynamic drag by the blood flow. In response to shear stress, EC as well as EC nuclei elongate, and nuclei orient themselves relative to the direction of flow. Nuclear positioning as well as alignment has previously been shown to be dependent on the association to microfilaments as well as the tubulin network ${ }^{29}$. In $\operatorname{amotl}^{\mathrm{ec}+/ \mathrm{ec}+}$ mice, nuclei of ECs of the DA were elongated and orientated in parallel with cell alignment in the direction of blood flow. However, in AmotL2 deficient ECs, the nuclei were more rounded with irregular shapes and positioned close to the cell edge downstream of the flow direction (Fig. 2g, quantification in $\mathbf{2 h}, \mathbf{i})$. These changes in nuclear shape and positioning were again not observed in the IVC (Fig. $\mathbf{2} \mathbf{j}$, quantification in $\mathbf{2} \mathbf{k}, \mathbf{l})$. Taken together, these data showed that AmotL2 is required for EC elongation as well as positioning of the EC nucleus.

\section{AmotL2 expression is required for arterial response to flow.}

Next we investigated whether AmotL2 is required for arterial endothelial compliance to laminar flow in vitro. For this purpose, we used a short hair-pin Lentiviral approach to deplete AmotL2 in Human Aortic ECs (HAoEC, Fig. 3a). No differences between control and AmotL2 depleted cells in cellular and nuclear shape were detectable under static 
conditions (Fig. 3b-d). To recapitulate arterial flow conditions, cells were exposed to 14 dynes $/ \mathrm{cm}^{2}$ for $48 \mathrm{~h}$ in a flow chamber as described in materials and methods. Control HAoECs exhibited an elongated phenotype and aligned in the direction of flow; however, the depletion of AmotL2 resulted in failure to elongate and align in the direction of flow (Fig. 3b, quantification in 3e,f). We could further show that AmotL2 was required for controlling nuclear shape and positioning. Consistent with the cellular shape change, nuclei also exhibited a rounder shape and could not maintain the positioning at the center of the cells when compared to control HAoECs (Fig. 3g,h).

\section{AmotL2 couples VE-cadherin to the nuclear lamina.}

Actin filaments are coupled to the nuclear membrane through the LINC complex ${ }^{29-32}$. This complex consisting of Sun-domain proteins (SUN-1 and -2) and KASH domain proteins (Nesprin-2) connects to Lamin A/C of the nuclear lamina. Therefore, we next investigated a possible connection between VE-cadherin, AmotL2, actin and the LINC complex.

We used a Co-IP approach to obtain AmotL2 associated immunocomplexes from both murine endothelial cell line (MS1) and primary Bovine Aortic Endothelial (BAE) cells. By spectrometry analysis (raw data in Supplementary Table 1,2), we identified cellular membrane protein VE-cadherin and $\alpha-, \beta$-, $\delta$-catenin, as well as the nuclear laminal proteins such as SUN2 and Lamin A, in all triplicate IP samples from MS1 cells (Fig. 4a). Consistently, the majority of key membrane proteins were recognized in BAE AmotL2 immunoprecipitates (Supplementary Fig. 3a). Mouse lungs consist of approximately 1020\% ECs. We performed immunoprecipitation using AmotL2 antibodies and could verify that Amotl2 associates with VE-cadherin, B-catenin, actin, and Lamin A also in the murine lung tissue in vivo (Fig. 4b). It was not possible to analyze Nesprin-2 by western blot due to its high molecular mass $(<800 \mathrm{kDa})$. The association of AmotL2 was dependent on 
myosin contraction and actin filaments as treatment with the myosin II inhibitor blebbistatin or the actin polymerization inhibitor cytochalasin $\mathrm{D}$ both decreased the association of AmotL2 to Lamin A/C and SUN2 (Fig. 4c). In addition, immunoprecipitation of VEcadherin confirmed the association to AmotL2 and Lamin A in HAoECs (Fig. 4d). Interestingly, shRNA depletion of AmotL2 protein in ECs abrogated the association of VEcadherin to both actin and Lamin A (Fig. 4d).

The higher expression levels of AmotL2 detected in the DA as compared to the IVC in vivo raised the question of whether AmotL2 associated complex is shear stress responsive. We placed primary Human Umbilical Venous ECs (HUVECs) in a $15 \mathrm{~cm}$ culture dish on an orbital shaker to apply the circulatory flow to the cells (schematic in Supplementary Fig. 3b). As previously described ${ }^{33}$, the alignment of the cells at the periphery area was observed after 96h (Supplementary Fig. 3c,d). Analyses of the AmotL2 IP revealed a dramatically increased binding with VE-cadherin, actin and nuclear membrane proteins compared to the static condition (Fig. 4e).

Taken together, MS and IP data suggest a model where VE-cadherin/AmotL2 forms a complex that is connected to the nuclear LINC complex via actin filaments (schematic in Fig.4f).

\section{Deletion of AmotL2 promotes vascular inflammation.}

EC alignment and cytoskeletal reorganization in response to laminar blood flow is protective against inflammation ${ }^{3}$. The change in the mechano-response of amotl2ec-/ec EC raised the question whether this was accompanied by a pro-inflammatory phenotype. The aortic arch is exposed to turbulent blood flow and the descending aorta is exposed to laminar flow. For this reason, we analyzed these areas of the aorta separately (Fig. 5a). mRNA isolated from DAs in both $\operatorname{amotl}^{\mathrm{ec}+/ \mathrm{ec}+}(\mathrm{n}=3)$ and $\operatorname{amotl}^{\mathrm{ec}-/ \mathrm{ec}-}(\mathrm{n}=5)$ mice were analyzed by 
mRNA-seq. Due to high variability between individual mice, only 65 genes were identified to be differentially expressed (adjusted $p$ value $<0.05$ ) between amotl2 $2^{e c+/ e c+}$ and amotl2 ${ }^{e c-}$ lec- groups (Supplementary Table 3). However, those genes were obviously enriched to immuno-related GO terms, such as "Neutrophil activation involved in immune response", "inflammatory response" and "regulation of immune effector process" (Fig. 5b). Gene expression profile analysis on MS1 cells in vitro treated with control or AmotL2 siRNA was also performed by RNA-seq. Interestingly, we did not observe any up-regulation of inflammatory gene signatures in vitro (Supplementary Fig. 4a and Supplementary Table 4), indicating that the in vivo environment was required for this to occur. We therefore performed whole-mount immunostaining to analyze the presence of CD45 ${ }^{+}$inflammatory cells in the aortic arch. The whole mount immunofluorescence staining showed the presence of spindle-like $\mathrm{CD}_{4} 5^{+}$cells in the inner curvature of the aortic arch in wild-type animals and in arterial bifurcations (Fig. 5c,d). Analyses of the amotl2ec-/ec- mice 1-month postinjection of tamoxifen revealed the infiltration of $\mathrm{CD} 45^{+}$round monocytes in the outer curvature of the aortic arch (Fig. 5c,d). The CD45 ${ }^{+}$monocyte-like cells were detected in the sub-endothelial compartment of the aorta (Fig. 5e,f). In total, 45\% (9/20) amotl2 $2^{\text {ec-/ec- }}$ arches contained more than one $\mathrm{CD} 45^{+}$lesion.

\section{Inflammatory markers are up-regulated in amotl $2^{\text {ec-lec- }}$ mouse aortae.}

Next, we quantified mRNA expression of inflammatory markers in DAs. The mRNA expression level analyzed by TaqMan qRT-PCR showed a significant increase of inflammatory markers, such as Il6, Tnf, and Cd68a in amotl2ec-/ec- DA, suggesting a provocation of a general immune response. Interestingly, up-regulation of pro-inflammatory markers was more pronounced in male animals (Fig. 5g-j). 
In response to pathogenic stimuli, expression of various leukocyte adhesion molecules can be augmented as the initial sign of vascular inflammation, such as ICAM-1 and VCAM-1. Indeed, in amotl $2^{\mathrm{ec}-\mathrm{ec}} \mathrm{DA}$, an increase in Vcam-1 expression on transcriptional level was detected by qPCR, but the change of Icam-1 was less obvious (Supplementary Fig. 4b,c), revealing the activation of endothelium of the DAs in the absence of AmotL2. Remarkably, Vcam-1 up-regulation was also more profound in male mice (Supplementary Fig. 4b). Once leukocytes attach and migrate into underlying intima, the process of lesion formation begins. This requires the participation of chemoattractant cytokines such as $\mathrm{Ccl} 2$, also known as monocyte chemoattractant protein-1 (MCP-1), which is especially important for the recruitment of mononuclear immune cells. Compared to the amotl $2^{e c+/ e c+}$ aortic tissues, amotl2 deletion caused an increase of $C c l 2$ expression in the descending regions (Fig. $\mathbf{5 j}$ ), as well as $\mathrm{Cxcl10}$, which is able to selectively attract NK cells as well as T and B lymphocytes. (Supplementary Fig. 4d). However, other chemokines such as Ccl5 had no significant changes (Supplementary Fig. 4e), suggesting that the induced inflammatory response was mainly affecting innate immune cells.

By contrast, the $\mathrm{T}$ and $\mathrm{B}$ cell markers $C d 4, C d 8$, and $C d 19$, as adaptive immune markers, showed no differences in expression level of these markers on average was detected in the DAs with or without AmotL2 (Supplementary Fig. 4f-h).

In conclusion, the expression profile of inflammatory-related genes indicates that AmotL2 depletion results in an innate immune response rather than an activation of adaptive immunity. Of note is the preferential upregulation of pro-inflammatory markers in amotl2 $2^{e c-}$ lec- males.

\footnotetext{
AmotL2 depletion promotes the formation of abdominal aortic aneurysms in male mice.
} 
Aortic inflammation is a predictor of the development of arterial aneurysm ${ }^{34}$. Inflammatory aortic aneurysms account for up to $10 \%$ of all AAA cases ${ }^{35}$. After dissection of the aorta, we could detect the formation of abdominal aortic aneurysms (AAA, dilatation>1.5 times normal size) in the proximity of the renal arterial branch (Fig. 6a), but not in ascending or thoracic aortae. Interestingly, $20 \%$ of male amotl $2^{\text {ec-lec- }}(5 / 25)$ mice developed an AAA; however, no aneurysm was detected in the females $(0 / 20)$. Furthermore, no aneurysms were observed in the amotl2 ${ }^{\mathrm{ec}+/ \mathrm{ec}+}$ mice (36 mice, 20 male and 16 female).

Imaging analysis of a typical AAA revealed damage to the endothelium as well as the vessel wall (Fig. 6b,c). The insult to the vessel wall was further verified by histological sectioning. The intima of the vessel wall was infiltrated by CD45+ leukocytes (Fig. 6d) and elastin fibers were degraded (Fig. 6e).

\section{AmotL2/YAP correlation in human AAA samples.}

Next, we assessed whether AmotL2 gene expression could be correlated to disease progression in human AAA patients. We analyzed mRNA expression in surgically resected materials from both healthy donors and patients diagnosed with AAA and undergoing aneurysm repair at the Karolinska University Hospital. mRNA samples were taken from both medial and adventitial layers of the intact aorta (13 donors) or AAA tissues (35 patients). Interestingly, AmotL2 expression level in the media tissue (containing endothelial cells, schematic in Fig. 7a) in AAA samples was significantly down regulated compared to the intact aortic media, which was not found in adventitia tissues (Fig. 7b). The expression pattern of the first exon of AmotL2 was specifically analyzed since this exon is specific for the p100-AmotL2 isoform that we have previously shown interacts with VE-cadherin ${ }^{25}$. In line with the studies in mice, there was an inverse correlation between the expression of the first exon of AMOTL2 and the luminal/external diameter of AAA (Fig. 7c). 
YAP (Yes Associated Protein 1) has been reported to act as a rheostat of external mechanical force ${ }^{36}$. Of interest is that YAP binds to the AmotL2 promoter and thus activates its transcription ${ }^{37}$. Consistent with these findings, a significant positive correlation was detected between AMOTL2 and YAP mRNA levels $(r=0.800, P<0.0001)$ in media tissues containing endothelium in AAA patients of both genders (Fig. 7d). We also found a weaker positive correlation of AMOTL2/YAP in adventitia tissues that did not contain ECs $(r=0.592, p=0.0002)$. A robust correlation of the well-established YAP target genes CTGF and CYR61 with AMOTL2 was also observed, as shown in Fig. 7e and 7f, respectively. CFOS has been reported to bind to the promoter and drive expression of the p60 AMOTL2 isoform. This isoform is primarily activated by severe hypoxia in ischemic tissues ${ }^{25}$. However, no correlation between AMOTL2 and C-FOS mRNA expression could be detected (Supplementary Fig. 5a). YAP and TAZ are paralogs with overlapping function as transcriptional regulators of the Hippo pathway. However, there was no significant correlation between AMOTL2 and TAZ expression, suggesting that YAP is primarily driving AMOTL2 expression in the human aortic tissues (Supplementary Fig. 5b). In addition, there was no correlation of AmotL2 with gender (Supplementary Fig. 5c-g). 


\section{Discussion}

The vascular endothelium plays an important role in the biomechanical response to hemodynamic forces. Understanding the pathways involved in this response is of importance to comprehend the pathogenesis of vascular disease. In this report we show for the first time that the cellular junctions of arterial ECs are connected via AmotL2 and microfilaments to the nuclear lamina. Interference with this pathway impairs EC alignment in response to shear stress and abrogates nuclear positioning resulting in inflammation and formation of abdominal aortic aneurysms.

We have used an inducible mouse model to target AmotL2 in the EC lineage. In our previous publication, we could show that Amotl2 silencing in ECs in utero resulted in impaired aortic expansion and death at embryonic day 10. Silencing of AmotL2 in adult mice, however, did not affect overall survival or had any obvious negative effects up to six months after AmotL2 depletion. The defect in adult mice was clearly more subtle as it was restricted to ECs exposed to arterial flow. We demonstrated not only that AmotL2 is required for cellular alignment in areas of shear stress, but also provided novel insights into how VE-cadherin is mechanically coupled to the cytoskeleton and thereby controls cell shape. AmotL2 triggers the formation of radial actin filaments that mediate junctional tension between neighboring cells. These radial actin filaments were detected in arterial, but not or at least at lower levels in venous endothelium. AmotL2 is a scaffold protein and as such brings together protein complexes of different functions such as Par3, MAGI-1b, Merlin, Actin, VE-cadherin. Of interest is that, in HUVECs of the venous origin, AmotL2 is sufficient to induce radial actin filaments when the cells aligned under flow condition. Our data are consistent with the notion that VE-cadherin is part of a mechanosensory complex with VEGFR2 and PECAM-1 as previously described ${ }^{38}$. The present data show that the VE-cadherin/AmotL2 protein complex is responsible for the actual cell shape 
modulation in arterial ECs. These most recent investigations together with our previous findings have shown that the VE-cadherin AmotL2 complex mediates mechanical forces between ECs, suggesting that AmotL2 may also relay mechanical forces not only from circulatory shear stress, but also transfers mechanical signals between cells. Of particular interest is the observation that AmotL2 is required for nuclear shape and subcellular positioning. Ingber and coworkers showed early on that there is a direct linkage between the cytoskeleton and the cell nucleus opening up the possibility of a novel mechanical signaling pathway from the exterior to the nucleus ${ }^{39}$. Actin filaments are directly associated with the nuclear lamina by binding to Nesprin2, SUN-1 and -2, and Lamin A that form the LINC complex. The actin filaments are anchored to the nuclear lamina via the LINC complex to form linear punctae called TAN lines. In this report we present evidence for a novel mechanical pathway that mechanically links junctional proteins to the nuclear LINC complex. We show that concomitant with the loss of AmotL2 and radial actin filaments, nuclei lose their central position and translocate to a polarized position in the cell, downstream of the exerted flow direction. Arterial nuclei not only lose their sub-cellular positioning, but the integrity of the nuclear lamina is perturbed. Measurement of forces exerted on the LINC proteins suggest that nuclear positioning is a result of the dynamic interactions of the cytoskeleton where nuclei are exposed to constant actomyosin forces ${ }^{40}$. Depletion of AmotL2 also had consequences for the integrity of the nuclear lamina. The nuclear lamina is an intermediate filament meshwork composed of two types of lamin proteins, the B type (Lamin B1 and B2) and the A type (Lamin A/C) and associated inner nuclear membrane proteins ${ }^{41}$. This network determines the mechanical properties and morphology of the nucleus ${ }^{42}$. In particular, Lamin A has been proposed to be responsive to mechanical cues from the extracellular matrix. Reduction of Lamin A in the nuclear membrane also results in a less rigid nuclear membrane. 
The lack of alignment and the consequent irregular shapes of the arterial endothelium concomitant with the loss of nuclear positioning had direct consequences for the endothelial function. We could show that AmotL2 deficient cells acquire a pro-inflammatory phenotype with ensuing formation of areas of vascular inflammation characterized by the presence of $\mathrm{CD}^{+} 5^{+}$monocytes. Quantitative PCR-analysis indicated a gender-specific difference in inflammatory response. The underlying reason is unclear but may relate to levels of estrogen as shown by other reports. Of clinical importance is the formation of AAA in the amotl2 $2^{\text {ec- }}$ lec- male mice. This is of interest as AAA has a relatively high prevalence in males between 65-79 years of age and the rupture of AAA is the cause of over 15000 deaths/year in the USA alone and 175000 globally $^{2,43,44}$. Our data indicate a lower expression of AmotL2 in AAA from patients as compared to healthy aortae. Furthermore, the expression levels of AmotL2 were negatively correlated with the diameter of the aneurysms. Several mouse models of AAA have been established for developing therapies for AAA. However, so far these models have failed to reliably predict results in clinical trials. Here we suggest a model where AmotL2 deficiency activates pro-inflammatory markers such as VCAM-1 and ICAM-1 on the EC surface. This promotes the extravasation of CD45+ inflammatory cells. The presence of inflammatory cells in the tunica media promotes the degradation of the extracellular matrix such as elastin, and thus weakens the physical strength of the aortic wall. The weakened area of the artery then bulges out and poses an increased risk of blood vessel rupture and hemorrhage (as shown in the schematic Fig. $\mathbf{7 g}$ ). The possibility that lower levels of AmotL2 could increase the risk of developing vascular inflammation, opens up the potential to therapeutically restore the AmotL2 mechano-transductory pathway and thereby increase the resilience of the arterial wall to shear stress. 


\section{Methods}

\section{Human material}

Aortic samples from AAA patients were obtained from the surgery performed at Karolinska Hospital in Stockholm. Control samples were taken from the abdominal aorta of beatingheart, solid organ transplant donors. Ethical permit (2009/1098-32) was granted by Regionala etikprövningsnämnden (Regional ethical review boards) in Stockholm. RNA was extracted from both medial and adventitia layer of the aortic wall and subsequently sequenced on HTA 2.0 (Human Transcriptome Array 2.0 - Affymetrix) platform.

\section{Mice and tamoxifen injections}

The amotl2floxfllox mice, carrying a loxP-flanked amotl2 gene, were crossed to

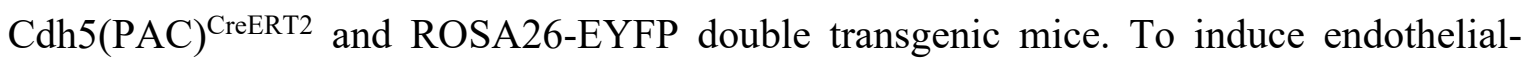
specific amotl 2 gene inactivation, tamoxifen was administered by intraperitoneal (IP) injection for 5 continuous days. For adult mice over 6 weeks old, $100 \mu$ l of tamoxifen $(20 \mathrm{mg} / \mathrm{ml})$ was administered at each injection site. Analysis of mice samples was performed four to six weeks after injections. All mice in this report were in C57BL/6 background, and both females and males were included. Ethical permits were obtained from North Stockholm Animal Ethical Committee and all experiments were carried out in accordance with the guidelines of the Swedish Board of Agriculture.

\section{Tissue preparation}

Mice were euthanized using carbon dioxide. Thoracic cavity was rapidly opened, and the heart was exposed while still beating. Cold PBS was injected through a cannula for perfusion for 1 minute and then changed to $4 \%$ paraformaldehyde (PFA) for another 1 minute perfusion. Aorta from root to aortic-common iliac bifurcation was dissected followed by careful removal of the connective tissues. After 1 h extra fixation in $4 \%$ PFA, 
the entire aorta was opened longitudinally. For the aortic arch part, using spring scissors, the inner curvature was cut along anteriorly, while the outer curvature of the aorta was opened from the aortic root through innominate, carotid, and subclavian arteries until the aortic arch resembled a Y shaped split. The whole flattened-out aorta was pinned onto the wax mold and prepared for immunostaining.

For RNA isolation, the aorta was perfused with cold PBS for 2 minutes before careful dissection. Thoracic and abdominal aorta were taken out and frozen in $-80^{\circ} \mathrm{C}$ for subsequent RNA extraction.

To isolate the urinary bladder, the mice were handled especially gently before sacrifice, which prevented urine leakage. Full bladders were immersed in fixative for 2 hours $(2 \mathrm{~h})$ and pinned on the wax mold in an open flower shape for whole-mount staining.

Mouse eyeballs were taken out at postnatal day 6 (P6) and month 6 (week 24). After a $2 \mathrm{~h}$ fixation in 4\% PFA, the retinas were dissected out and prepared for immunofluorescence staining for analysis of vasculature.

\section{Cell culture}

MS1 cells were cultured in RPMI-1640 medium (GIBCO, 21875-034) supplemented with 10\% FBS (GIBCO, 10270-106) and 1\% penicillin/streptomycin (P/S, GIBCO, 15140-122). BAE cells (Bovine Aortic Endothelial cell) purchased from Sigma-Aldrich (B304-05) were cultured in Bovine Endothelial Cell Growth Medium (Sigma, B211-500). Human Umbilical Vein Endothelial Cells (HUVEC) purchased from ScienCell (\#8000) were cultured in Endothelial cell Medium (ScienCell, \#1001). Human Aortic Endothelial Cell (HAoEC) were purchased from PromoCell (C-12271) and cultured with Endothelial Cell Growth Medium MV (C-22020). To note, the batch of HAoEC used for this study was from a 55year-old male donor with a Caucasian background.

\section{Lentiviral induced knock-down}


For knockdown studies, HAoECs (PromoCell) were infected with customized AmotL2 shRNA Lentiviral particles (Sigma) or scrambled shRNA control virus in complete endothelial cell medium with $5 \mu \mathrm{g} / \mathrm{mL}$ polybrene (Vector Builder). The Lentiviruscontaining medium was removed after overnight incubation and fresh medium was added. Further analyses of confluent cells (WB, IF, Ibidi flow) were performed at $\geq 72 \mathrm{~h}$ after infection.

\section{Flow experiments}

Ibidi flow system: Flow chamber slides (Ibidi $\mu$-Slides VI 0,4 Ibidi-treated) with a volume of $30 \mu \mathrm{l}$ per parallel channel were coated with fibronectin (Sigma). HAoECs/HUVECs were grown on the slides for $24 \mathrm{~h}$ until $30-50 \%$ confluency followed by infection with Lentivirus or control treatment. $96 \mathrm{~h}$ after that cells were subjected to $14 \mathrm{dyn} / \mathrm{cm}^{2}$ laminar flow using the Ibidi pump system and pump control software or kept at static conditions and cultured for $48 \mathrm{~h}$ at $37^{\circ} \mathrm{C} 5 \% \mathrm{CO}_{2}$. Cells were then harvested and processed for further analysis.

Orbital shaker: The Rotamax 120 (Heidolph) complies well with cell incubator and generates circular motion with the maximum speed of $300 \mathrm{rpm}$. HUVECs on a $15 \mathrm{~cm}$ culture dish with $16 \mathrm{ml}$ medium were placed on the shaker for $96 \mathrm{~h}$ before the harvesting the cell lysates.

\section{Immunofluorescence (IF) staining}

Immunofluorescence staining on MS1 cells, HAoEC, and HUVECs was performed as previously described ${ }^{25}$. To stain open aorta pinned on wax, endothelium exposed on the top layer was carefully treated using the same protocol as for cell staining, with the exception that the aorta was permeabilized for 20 minutes with $0.1 \%$ Tween 20 in PBS.

Retinas and bladders were blocked and permeabilized in 1\% BSA and 0.3\% Triton-X100 in PBS overnight. Pblec buffer (1.0\% Triton X-100 plus 0.1M MgCl2, 0.1M CaCl2, $0.01 \mathrm{M}$ $\mathrm{MnCl} 2$ in PBS) was used for washing and incubating one or more primary antibodies. Then 
fluorophore-conjugated antibodies were added in blocking buffer, followed by 5 x 20minutes wash with blocking buffer at 1:1 dilution in PBS. The cells/whole tissue was finally mounted with Fluoroshield ${ }^{\mathrm{TM}}$ with DAPI (Sigma, F6057). For a list of primary and secondary antibodies used see Supplementary Table 5.

\section{Western blot (WB)}

Cells were scraped directly from the culture dish in lysis buffer (50 mM Hepes buffer, 150 $\mathrm{mM} \mathrm{NaCl}, 1.5 \mathrm{mM} \mathrm{MgCl} 2,1 \mathrm{mM}$ EGTA, 10\% Glycerol, 1\% TritonX-100), with $1 \mathrm{x}$ protease inhibitor (Roche, 04693159001) and optionally with Phosphatase Inhibitor Cocktail 1 (Sigma, P2850). Lysates were prepared with SDS sample buffer (Novex, 1225644) containing $10 \%$ sample reducing agent (Novex, 1176192), separated in a polyacrylamide gel with $4-12 \%$ gradient (Novex, NP0322BOX), and transferred to a nitrocelluose membrane (Whatman, 10401396). The membrane was blocked in 5\% non-fat milk PBS with $0.1 \%$ Tween 20 and sequentially incubated with the primary antibody at $4{ }^{\circ} \mathrm{C}$ overnight. Horseradish peroxidase (HRP) conjugated secondary antibody was added in order for labelled proteins to be detected by Western Lightning Plus-ECL (PerkinElmer, 203-170071). Primary and secondary antibodies used are listed in Supplementary Table 5.

\section{Co-immunoprecipitation (Co-IP) analysis}

Mouse lung tissues were cut into small pieces before transferring into lysis buffer for western blot. Tissue homogenizer was gently applied for better protein extraction. Cell/tissue lysates were incubated with protein G sepharose beads (GE, 17-0618-01) for 1.5 hours at $4^{\circ} \mathrm{C}$ as pre-cleaning. Afterwards $2 \mu \mathrm{g}$ Amot12, Lamin $\mathrm{A} / \mathrm{C}$, VE-cadherin antibody or control IgG were added in the lysates overnight at $4^{\circ} \mathrm{C}$. The next morning, the immunocomplexes were enriched by protein $\mathrm{G}$ beads for $2 \mathrm{~h}$ at $4^{\circ} \mathrm{C}$, followed by five-time washing with lysis buffer. The final protein samples were fractionated by polyacrylamide gel and fractions were western blotted for evaluation of IP protein input level. 


\section{Mass spectrometry (MS) analysis}

IP samples were dissolved in $200 \mu \mathrm{L}$ lysis buffer (4 \% SDS, $50 \mathrm{mM}$ HEPES pH 7.6, $1 \mathrm{mM}$ DTT). After heating at $95^{\circ} \mathrm{C}$ for $5 \mathrm{~min}$ and centrifugation at $14,000 \mathrm{~g}$ for $15 \mathrm{~min}$, the supernatant was transferred to new tubes. Protein digestion was performed using a modified SP3-protocol. In brief, each sample was alkylated with $40 \mathrm{mM}$ chloroacetamide and SeraMag SP3 bead mix $(20 \mu 1)$ was transferred into the protein sample together with $100 \%$ acetonitrile to a final concentration of $70 \%$. The mix was incubated under rotation at room temperature for $20 \mathrm{~min}$. The mixture was placed on the magnetic rack and the supernatant was discarded, followed by two washes with $70 \%$ ethanol and one with $100 \%$ acetonitrile. The beads-protein-complex was reconstituted in $100 \mu 1$ trypsin buffer (50 mM HEPES pH 7.6 and $0.8 \mu \mathrm{g}$ trypsin) and incubated overnight at $37^{\circ} \mathrm{C}$. The eluted samples were dried in a SpeedVac. Approximately $10 \mu \mathrm{g}$ was suspended in LC mobile phase A and $1 \mu \mathrm{g}$ was injected on the LC-MS/MS system.

Online LC-MS was performed using a Dionex UltiMateTM 3000 RSLCnano System coupled to a Q-Exactive-HF mass spectrometer (Thermo Scientific). IP samples were trapped on a C18 guard-desalting column (Acclaim PepMap 100, $75 \mu \mathrm{m}$ x 2cm, nanoViper, C18, $5 \mu \mathrm{m}$, $100 \AA$ ), and separated on a 50cm long C18 column (Easy spray PepMap RSLC, C18, $2 \mu \mathrm{m}$, $100 \AA, 75 \mu \mathrm{m} \times 50 \mathrm{~cm})$. The nano capillary solvent A was $95 \%$ water, $5 \%$ DMSO, $0.1 \%$ formic acid; and solvent B was 5\% water, 5\% DMSO, 95\% acetonitrile, 0.1\% formic acid. At a constant flow of $0.25 \mu \mathrm{min}^{-1}$, the curved gradient went from $2 \% \mathrm{~B}$ up to $40 \% \mathrm{~B}$ in $240 \mathrm{~min}$, followed by a steep increase to $100 \% \mathrm{~B}$ in $5 \mathrm{~min}$.

FTMS master scans with 70,000 resolution (and mass range 300-1700 m/z) were followed by data-dependent MS/MS (35,000 resolution) on the top 5 ions using higher-energy collision dissociation (HCD) at 30-40 \% normalized collision energy. Precursors were isolated with a $2 \mathrm{~m} / \mathrm{z}$ window. Automatic gain control (AGC) targets were $1 \mathrm{e}^{6}$ for MS1 and 
$1 \mathrm{e}^{5}$ for MS2. Maximum injection times were $100 \mathrm{~ms}$ for MS1 and 150-200 ms for MS2. The entire duty cycle lasted $\sim 2.5 \mathrm{~s}$. Dynamic exclusion was used with 60 s duration. Precursors with unassigned charge state or charge state 1 were excluded and an underfill ratio of $1 \%$ was used.

\section{Quantitative real-time RT-PCR (qRT-PCR)}

To extract RNA from dissected aorta from $\operatorname{amotl}^{\mathrm{ec}+/ \mathrm{ec}+}(\mathrm{n}=3)$ and $\operatorname{amotl}^{\mathrm{ec}-\mathrm{ec}-}$ mice $(\mathrm{n}=3)$ was immersed in TRIzol and homogenised by TissueLyser (Qiagen) in TRIzol. Chloroform addition allowed the homogenate to separate into the lower organic phase and the upper clear aqueous phase (containing RNA). MS1 cells were scraped directly from culture dish in RLT buffer. Total RNA purification was carried out using RNeasy Plus Mini Kit (Qiagen), after which RNA concentrations were measured by Nanodrop spectrophotometer (ThermoFisher Scientific). cDNA synthesis was performed using the High-Capacity cDNA Reverse Transcription Kit (Applied Biosystems). Quantitive real-time PCR was performed on a 7900HT Fast Real-Time PCR system (Perkin-Elmer Applied Biosystems) using TaqMan Assay-on-Demand (Applied Biosystems). Results were calculated as $2-\Delta \mathrm{CT}$ obtained by comparing the threshold cycle (CT) for the genes of interest with that obtained for housekeeping gene hypoxanthine guanine phosphoribosyltransferase (HPRT). All TaqMan probes involved were purchased from Thermo Fisher Scientific and detailed information can be found in Supplementary Table 5.

\section{RNA-seq}

Total RNA purified from full-length aorta (thoracic and abdominal aorta) of amotl2 $2^{\mathrm{ec} / \mathrm{ec}+}$ $(n=3)$ and amotl2 $2^{\text {ec-lec- }}$ mice $(n=3)$ were sent for RNA-seq analysis (Novogene, Beijing, China). Libraries were prepared from $4-5 \mu \mathrm{g}$ of total RNA. PolyA RNA was purified using the Dynabeads mRNA purification kit (Ambion) and fragmented using Fragmentation Reagent (Ambion). First strand cDNA was synthesized from polyA RNA using the 
SuperScript III Reverse Transcriptase Kit with random primers (Life Technologies). Second strand cDNA synthesis was performed using Second Strand Synthesis buffer, DNA Pol I, and RNase H (Life Technologies). cDNA libraries were prepared for sequencing using the mRNA TruSeq protocol (Illumina).

The genes with significantly differential expression were input to online Enrichr (Ma'ayan Laboratory, Computational systems biology) for GO term analysis (Biological process $2018)^{45,46}$

\section{Statistical analysis}

All statistical figures and analyses were performed using GraphPad Prism software, with the exception of the gene correlation graphs, which were generated using R (https://www.rproject.org/index.html). Statistical analysis of in vivo results was based on at least three animals per group. Comparisons between two groups with similar variances were analyzed by the standard unpaired two-tailed Student t-test, while comparisons between multiple groups were analyzed by Kruskal-Wallis test. The correlation between two genes were analyzed by Pearson Correlation and pearson correlation coefficient is referred to $r$. A value of $P<0.05$ was considered as statistically significant (n.s. not significant, ${ }^{*} P<0.05,{ }^{* *} P<0.01$ and $\left.{ }^{* * *} P<0.001\right)$. No statistical method was used to predetermine sample size in animal studies and the experiments were not randomized. The investigators were not blinded to allocation during experiments and outcome assessment.

\section{Acknowledgments}

We are indebted to Dr. Ralf H Adams, University of Münster, who kindly provided the Cdh5(PAC)-CreER ${ }^{\mathrm{T} 2}$ and ROSA26-EYFP transgenic mice. Many thanks to Lynn Butler and Philip Dusart for letting us perform experiments using their Ibidi Flow System at the 
SciLifeLab, Stockholm, Sweden. The paraffin sections and IHC staining of the aortae were performed by Anna Malmerfelt in histology core facility at the Department of Oncology and Pathology, Karolinska Institute, Sweden. We are grateful for the polar bar chart coded by freelance programmer Andreas Gustafsson using Python.

This study was supported by grants from the Swedish Heart and Lung Foundation, Novo Nordisk Foundation (NNF15CC0018346) the Swedish Cancer Society, the Swedish Childhood Cancer Foundation, Cancer Society of Stockholm, the Swedish Research Council and Knut and Alice Wallenberg Foundation.

\section{Author contributions}

Y.Z. performed in vivo experiments using the AmotL2 transgenic model, performed in vitro experiments, finalized all figures, and wrote the manuscript. E.H. performed Lenti-virus based transfection in vitro and Ibidi flow experiment with data analysis. S.H. performed in vivo experiments on the Amotl2 mouse model and summarized data. O.B. and P.E. performed bio-informatical analysis on human aortic samples. J.R. contributed with human samples from clinical surgery. D.K. designed the experiments to investigate inflammation and M.F. performed the TaqMan qPCR on RNA from murine aortic samples. Z.A. performed flow experiment with orbital shaker. L.H developed the theory, designed experiments and wrote the manuscript. All authors reviewed the manuscript prior to submission.

\section{Competing financial interests}

The authors declare no competing financial interests. 


\section{Reference}

1 Boyd, A. J., Kuhn, D. C., Lozowy, R. J. \& Kulbisky, G. P. Low wall shear stress predominates at sites of abdominal aortic aneurysm rupture. J Vasc Surg 63, 16131619, doi:10.1016/j.jvs.2015.01.040 (2016).

2 KC, K. et al. Analysis of risk factors for abdominal aortic aneurysm in a cohort of more than 3 million individuals. Journal of vascular surgery $\mathbf{5 2}$, doi:10.1016/j.jvs.2010.05.090 (2010).

3 O, T. \& BC, B. Laminar shear stress: mechanisms by which endothelial cells transduce an atheroprotective force. Arteriosclerosis, thrombosis, and vascular biology 18, doi:10.1161/01.atv.18.5.677 (1998).

4 Baeyens, N., Bandyopadhyay, C., Coon, B. G., Yun, S. \& Schwartz, M. A. Endothelial fluid shear stress sensing in vascular health and disease. The Journal of clinical investigation 126, 821-828, doi:10.1172/jci83083 (2016).

5 Davies, P. F. Hemodynamic shear stress and the endothelium in cardiovascular pathophysiology. Nature clinical practice. Cardiovascular medicine 6, 16-26, doi:10.1038/ncpcardio1397 (2009).

6 Garcia-Cardena, G., Comander, J., Anderson, K. R., Blackman, B. R. \& Gimbrone, M. A., Jr. Biomechanical activation of vascular endothelium as a determinant of its functional phenotype. Proceedings of the National Academy of Sciences of the United States of America 98, 4478-4485, doi:10.1073/pnas.071052598 (2001).

7 McCue, S. et al. Shear stress regulates forward and reverse planar cell polarity of vascular endothelium in vivo and in vitro. Circulation research 98, 939-946, doi:10.1161/01.Res.0000216595.15868.55 (2006).

8 Levesque, M. J. \& Nerem, R. M. The elongation and orientation of cultured endothelial cells in response to shear stress. Journal of biomechanical engineering 107, 341-347, doi:10.1115/1.3138567 (1985).

9 Noria, S., Cowan, D. B., Gotlieb, A. I. \& Langille, B. L. Transient and steady-state effects of shear stress on endothelial cell adherens junctions. Circulation research 85, 504-514, doi:10.1161/01.res.85.6.504 (1999).

10 Seebach, J. et al. Regulation of endothelial barrier function during flow-induced conversion to an arterial phenotype. Cardiovascular research 75, 596-607, doi:10.1016/j.cardiores.2007.04.017 (2007).

11 White, C. R. \& Frangos, J. A. The shear stress of it all: the cell membrane and mechanochemical transduction. Philosophical transactions of the Royal Society of London. Series B, Biological sciences 362, 1459-1467, doi:10.1098/rstb.2007.2128 (2007).

12 Hahn, C. \& Schwartz, M. A. Mechanotransduction in vascular physiology and atherogenesis. Nat Rev Mol Cell Biol 10, 53-62, doi:10.1038/nrm2596 (2009).

13 Bryan, M. T. et al. Mechanoresponsive networks controlling vascular inflammation. Arteriosclerosis, thrombosis, and vascular biology 34, 2199-2205, doi:10.1161/atvbaha.114.303424 (2014).

14 Davies, P. F., Civelek, M., Fang, Y. \& Fleming, I. The atherosusceptible endothelium: endothelial phenotypes in complex haemodynamic shear stress regions in vivo. Cardiovascular research 99, 315-327, doi:10.1093/cvr/cvt101 (2013).

15 Dai, G. et al. Distinct endothelial phenotypes evoked by arterial waveforms derived from atherosclerosis-susceptible and -resistant regions of human 
vasculature. Proceedings of the National Academy of Sciences of the United States of America 101, 14871-14876, doi:10.1073/pnas.0406073101 (2004).

16 Chien, S. Effects of disturbed flow on endothelial cells. Annals of biomedical engineering 36, 554-562, doi:10.1007/s10439-007-9426-3 (2008).

17 Malek, A. M., Alper, S. L. \& Izumo, S. Hemodynamic shear stress and its role in atherosclerosis. Jama 282, 2035-2042, doi:10.1001/jama.282.21.2035 (1999).

18 Tzima, E. et al. A mechanosensory complex that mediates the endothelial cell response to fluid shear stress. Nature 437, 426-431, doi:10.1038/nature03952 (2005).

19 Charbonier, F. W., Zamani, M. \& Huang, N. F. Endothelial Cell Mechanotransduction in the Dynamic Vascular Environment. Advanced biosystems 3, doi:10.1002/adbi.201800252 (2019).

20 Bratt, A. et al. Angiomotin belongs to a novel protein family with conserved coiled-coil and PDZ binding domains. Gene 298, 69-77, doi:10.1016/s03781119(02)00928-9 (2002).

21 Wells, C. D. et al. A Rich1/Amot complex regulates the Cdc42 GTPase and apical-polarity proteins in epithelial cells. Cell 125, 535-548, doi:10.1016/j.cell.2006.02.045 (2006).

22 Aase, K. et al. Angiomotin regulates endothelial cell migration during embryonic angiogenesis. Genes Dev 21, 2055-2068, doi:10.1101/gad.432007 (2007).

23 Yi, C. et al. A tight junction-associated Merlin-angiomotin complex mediates Merlin's regulation of mitogenic signaling and tumor suppressive functions. Cancer cell 19, 527-540, doi:10.1016/j.ccr.2011.02.017 (2011).

24 Ernkvist, M. et al. The Amot/Patj/Syx signaling complex spatially controls RhoA GTPase activity in migrating endothelial cells. Blood 113, 244-253, doi:10.1182/blood-2008-04-153874 (2009).

25 Hultin, S. et al. AmotL2 links VE-cadherin to contractile actin fibres necessary for aortic lumen expansion. Nat Commun 5, 3743, doi:10.1038/ncomms4743 (2014).

26 Zheng, Y. et al. Angiomotin like-1 is a novel component of the N-cadherin complex affecting endothelial/pericyte interaction in normal and tumor angiogenesis. Scientific reports 6, 30622, doi:10.1038/srep30622 (2016).

27 Hildebrand, S. et al. The E-cadherin/AmotL2 complex organizes actin filaments required for epithelial hexagonal packing and blastocyst hatching. Scientific reports 7, 9540, doi:10.1038/s41598-017-10102-w (2017).

28 Pitulescu, M. E., Schmidt, I., Benedito, R. \& Adams, R. H. Inducible gene targeting in the neonatal vasculature and analysis of retinal angiogenesis in mice. Nature protocols 5, 1518-1534, doi:10.1038/nprot.2010.113 (2010).

29 Gundersen, G. G. \& Worman, H. J. Nuclear positioning. Cell 152, 1376-1389, doi:10.1016/j.cell.2013.02.031 (2013).

30 Chang, W., Antoku, S., Ostlund, C., Worman, H. J. \& Gundersen, G. G. Linker of nucleoskeleton and cytoskeleton (LINC) complex-mediated actin-dependent nuclear positioning orients centrosomes in migrating myoblasts. Nucleus (Austin, Tex.) 6, 77-88, doi:10.1080/19491034.2015.1004947 (2015).

31 Lee, Y. L. \& Burke, B. LINC complexes and nuclear positioning. Seminars in cell \& developmental biology 82, 67-76, doi:10.1016/j.semcdb.2017.11.008 (2018).

32 Zhu, R., Antoku, S. \& Gundersen, G. G. Centrifugal Displacement of Nuclei Reveals Multiple LINC Complex Mechanisms for Homeostatic Nuclear Positioning. Curr Biol 27, 3097-3110.e3095, doi:10.1016/j.cub.2017.08.073 (2017). 
33 A, D. et al. Differential effects of orbital and laminar shear stress on endothelial cells. Journal of vascular surgery 41, doi:10.1016/j.jvs.2005.01.020 (2005).

34 Gornik, H. L. \& Creager, M. A. Aortitis. doi:10.1161/CIRCULATIONAHA.107.760686 (2008).

35 Hellmann, D. B., Grand, D. J. \& Freischlag, J. A. Inflammatory Abdominal Aortic Aneurysm. JAMA 297, 395-400, doi:10.1001/jama.297.4.395 (2007).

36 Panciera, T., Azzolin, L., Cordenonsi, M. \& Piccolo, S. Mechanobiology of YAP and TAZ in physiology and disease. Nature Reviews Molecular Cell Biology 18, 758-770, doi:doi:10.1038/nrm.2017.87 (2017).

37 Grijalva, J. L. et al. Dynamic alterations in Hippo signaling pathway and YAP activation during liver regeneration. American journal of physiology.

Gastrointestinal and liver physiology 307, G196-204, doi:10.1152/ajpgi.00077.2014 (2014).

38 Conway, D. E. et al. VE-Cadherin Phosphorylation Regulates Endothelial Fluid Shear Stress Responses through the Polarity Protein LGN. Curr Biol 27, 22192225.e2215, doi:10.1016/j.cub.2017.06.020 (2017).

39 Yang, J. H., Sklar, P., Axel, R. \& Maniatis, T. Purification and characterization of a human RNA adenosine deaminase for glutamate receptor B pre-mRNA editing. Proceedings of the National Academy of Sciences of the United States of America 94, 4354-4359, doi:10.1073/pnas.94.9.4354 (1997).

40 Mayer, C. R., Arsenovic, P. T., Bathula, K., Denis, K. B. \& Conway, D. E. Characterization of 3D Printed Stretching Devices for Imaging Force Transmission in Live-Cells. Cellular and molecular bioengineering 12, 289-300, doi:10.1007/s12195-019-00579-y (2019).

41 Gruenbaum, Y., Margalit, A., Goldman, R. D., Shumaker, D. K. \& Wilson, K. L. The nuclear lamina comes of age. Nature reviews. Molecular cell biology 6, 21-31, doi:10.1038/nrm1550 (2005).

42 Gruenbaum, Y. \& Foisner, R. Lamins: nuclear intermediate filament proteins with fundamental functions in nuclear mechanics and genome regulation. Annual review of biochemistry 84, 131-164, doi:10.1146/annurev-biochem-060614034115 (2015).

43 PW, S. et al. A review of current reporting of abdominal aortic aneurysm mortality and prevalence in the literature. European journal of vascular and endovascular surgery : the official journal of the European Society for Vascular Surgery 47, doi:10.1016/j.ejvs.2013.11.007 (2014).

44 DP, H. et al. Population-Based Study of Incidence of Acute Abdominal Aortic Aneurysms With Projected Impact of Screening Strategy. Journal of the American Heart Association 4, doi:10.1161/JAHA.115.001926 (2015).

45 Chen, E. Y. et al. Enrichr: interactive and collaborative HTML5 gene list enrichment analysis tool. BMC bioinformatics 14, 128, doi:10.1186/1471-2105-14128 (2013).

46 Kuleshov, M. V. et al. Enrichr: a comprehensive gene set enrichment analysis web server 2016 update. Nucleic acids research 44, W90-97, doi:10.1093/nar/gkw377 (2016). 
a

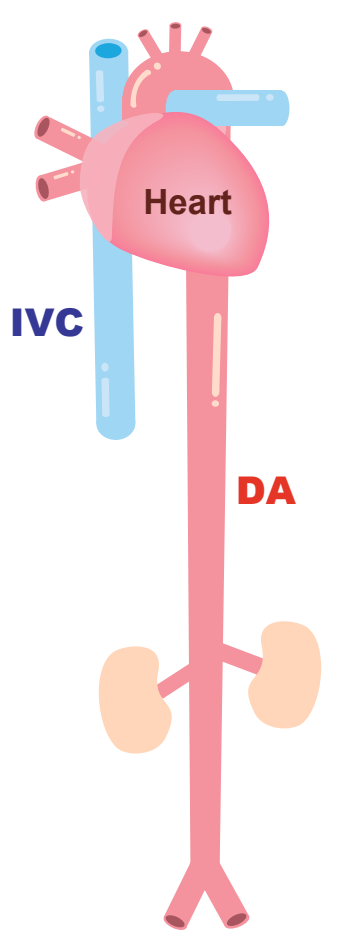

b

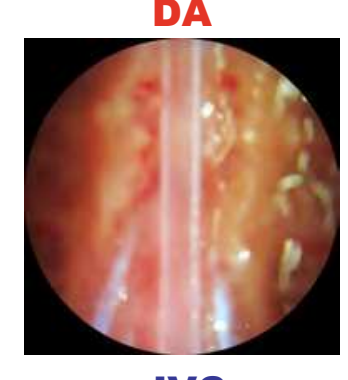

IVC

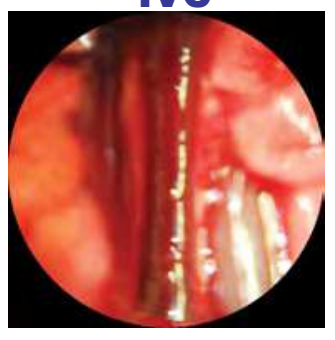

d

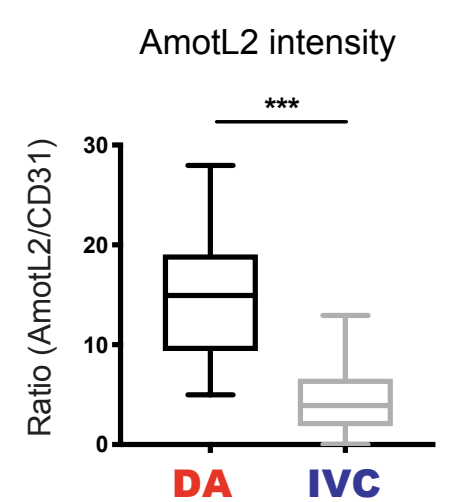

C
AmotL2
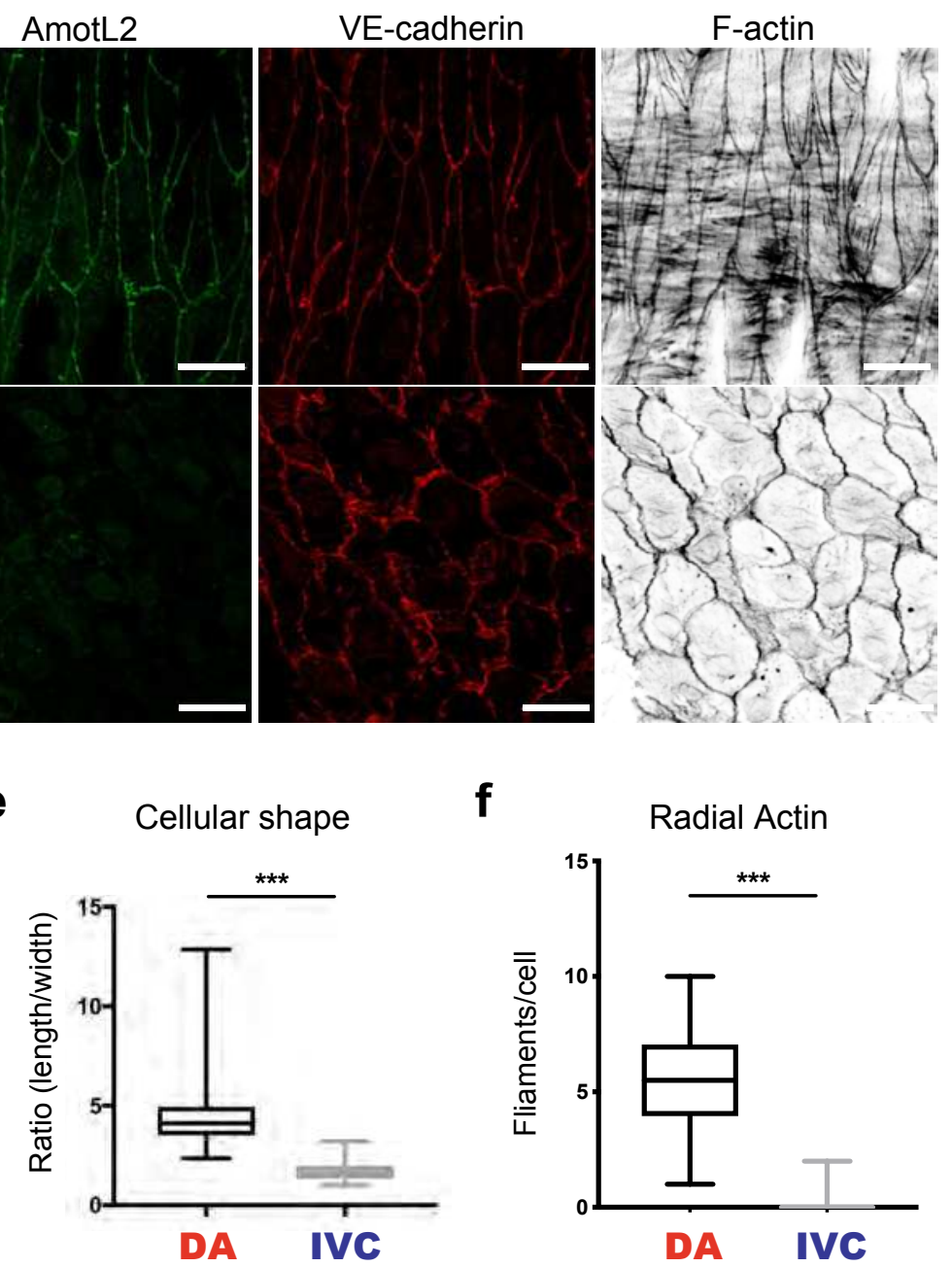

Radial Actin

f

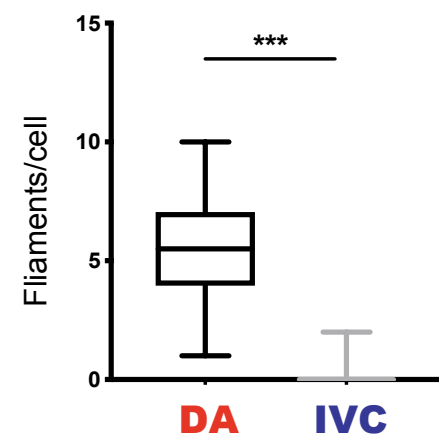


Figure 1. AmotL2 is primarily expressed in DA rather than IVC. a, Schematic of major blood vessels connected to the heart. DA indicates descending aorta leaving from the heart and IVC is for inferior vena cava. b, Anatomical images of the DA and IVC under microscopy during dissection process. DA image was taken after PFA perfusion. c, Representative images of DA and IVC used for whole-mount staining. Both vessels were stained with immunoaffinity purified AmotL2 antibodies (in green), VE-cadherin (in red) and phalloidin (in gray scale). d, Graph of box plot showing quantification of fluorescence intensity of AmotL2 in co-localization with CD31 in amotl2 $^{\mathrm{ec}+/ \mathrm{ec}+}$ DA, using ImageJ. e, Box plot depicting the difference of cell length/width ratio in amotl2 $^{\mathrm{ec}+/ \mathrm{ec}+}$ DA and IVC. f, Quantification of macroscopic radial actin filaments per EC in the DA and IVC. At least 4 images per mouse ( $\mathrm{n}>3$ ) were analyzed for each group. ${ }^{* * *} P<0.001$. Scale bars, $20 \mu \mathrm{m}$. 


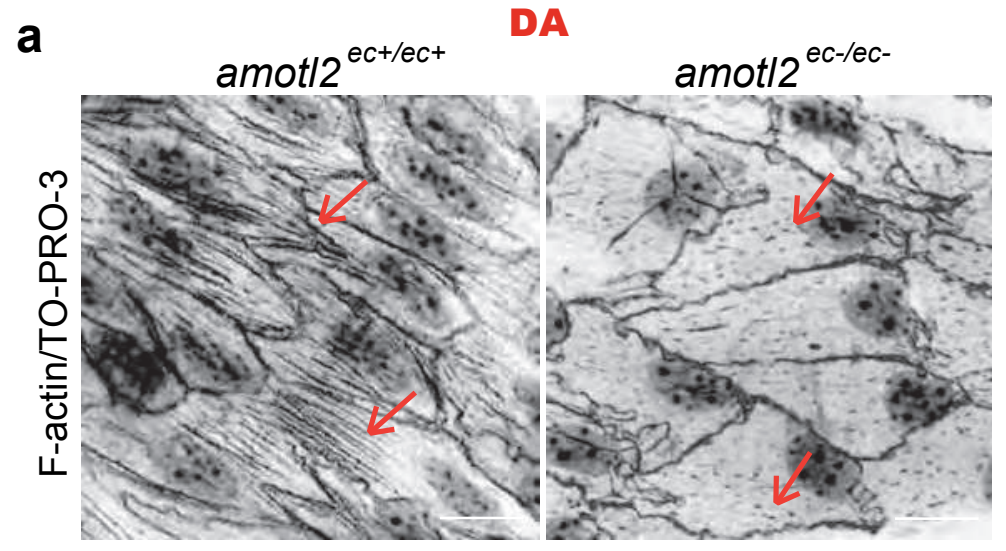

d

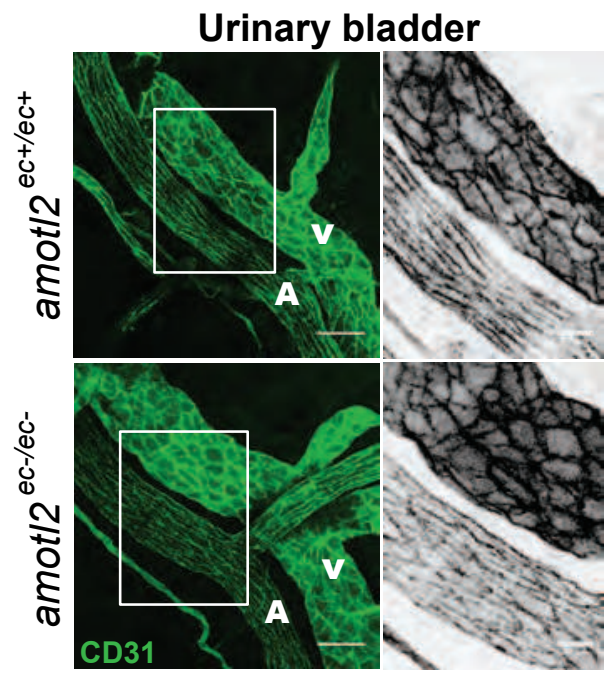

g

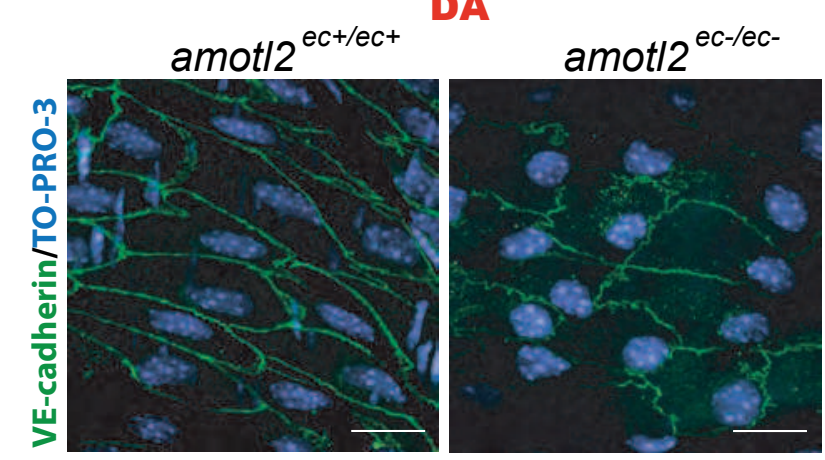

j

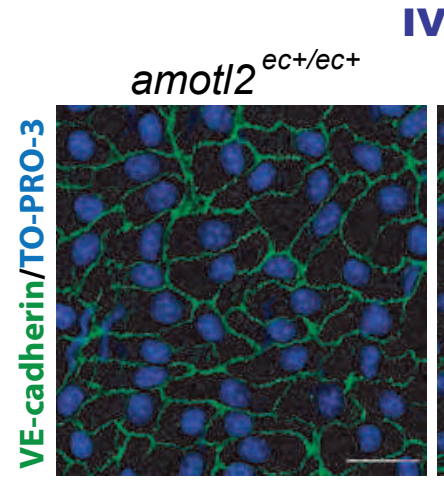

b Radial actin
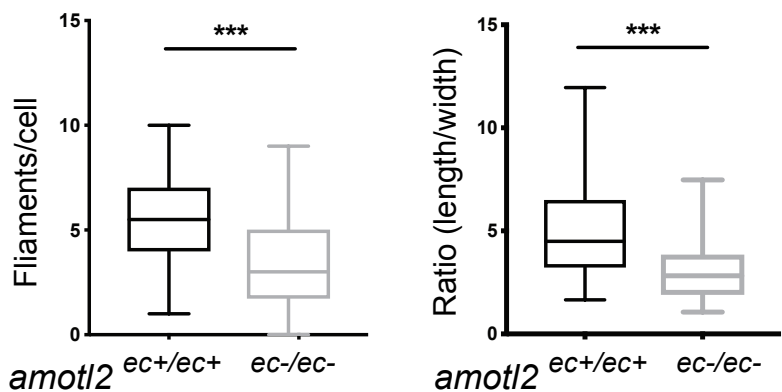

e

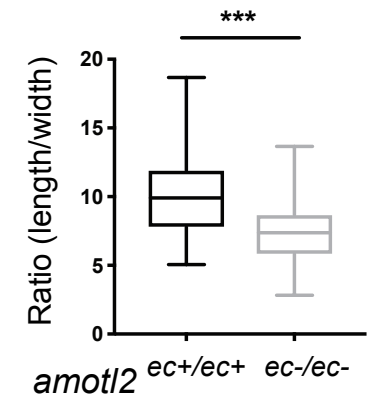

h Nuclear shape

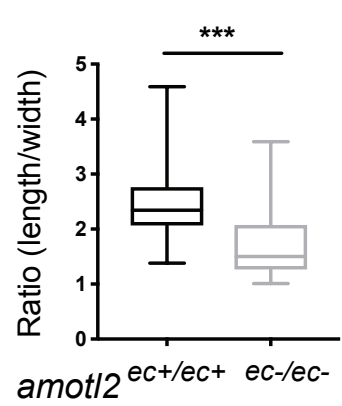

k

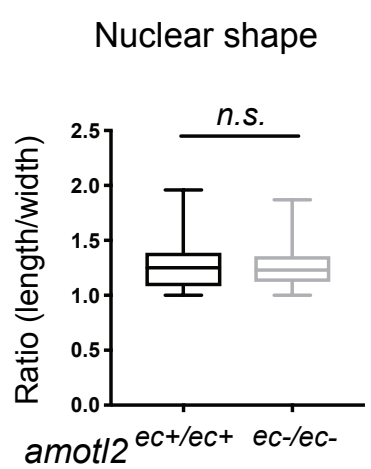

Veins

Cellular shape
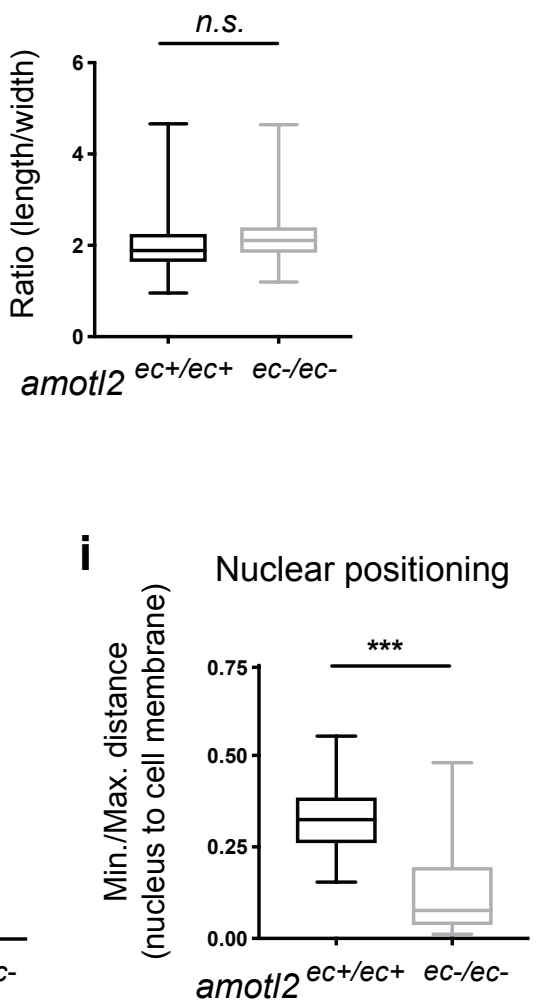

I

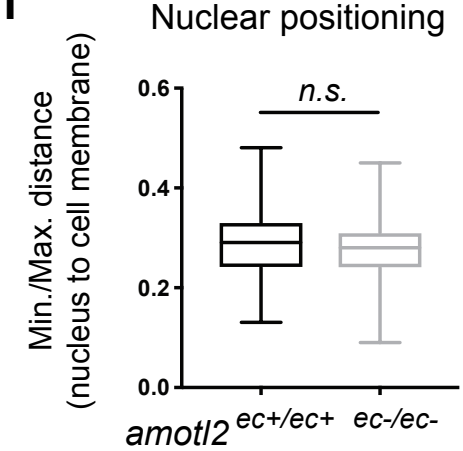


Figure 2. Deletion of AmotL2 resulted in aortic endothelial cellular and nuclear shape change. a, Whole-mount IF staining of amotl $^{\mathrm{ec}+/ \mathrm{ec}+}$ and amotl $^{\mathrm{ec}-/ \mathrm{ec}-}$ DA with phalloidin and TOPRO-3 showing F-actin and nuclei (in gray scale), respectively. b, Box plot showing quantification of radial actin filaments per EC in DAs from amotl $2^{\mathrm{ec}+/ \mathrm{ec}+}$ and amotl $^{\mathrm{ec}-/ \mathrm{ec}-}$ mice ( $\mathrm{n}=6$ in each group). c, Quantification of cell length/width ratio in amotl2 $\mathrm{ec}^{\mathrm{ec} / \mathrm{ec}+}$ and amotl2 $^{\mathrm{ec}-/ \mathrm{ec}-}$ DAs ( $n=6$ in each group). d. IF staining of bladder vasculature in $a m o t l 2^{\mathrm{ec}+/ \mathrm{ec}+}(n=6)$ and $a m o t l 2^{\mathrm{ec}-}$ /ec- $(n=6)$ mice, with PECAM-1 indicating ECs (in green). Quantification of EC shape in bladder arteries and veins were presented in e and $\mathbf{f}$, respectively. Boxed area in the left panel was magnified in the middle and right panels. White character $\mathrm{V}$ and $\mathrm{A}$ were abbreviations of vein and artery respectively. g, Whole-mount IF staining of amotl $2^{\mathrm{ec}+/ \mathrm{ec}+}$ and amotl2 $2^{\mathrm{ec}-/ \mathrm{ec}-}$ DAs with VEcadherin (in green) and TO-PRO-3 showing nuclei (in blue,). h, Quantification of nuclear length/width ratio in amotl2 ${ }^{e c+/ e c+}$ and $\operatorname{amotl}^{e c-/ e c-}$ DAs ( $\mathrm{n} \geq 6$ in each group). Along with the long axis of the EC, the closest distance between nuclei to one end of the cell was measured and normalized to the full cell length. The ratio was depicted in box plot i. j, Representative images on IVC of amotl2 $2^{\mathrm{ec}+/ \mathrm{ec}+}$ and amotl2 $2^{\mathrm{ec}-/ \mathrm{ec}-}$ mice with VE-cadherin (in green) and TO-PRO-3 (in blue). Quantification of nuclear shape and nuclear positioning of ECs in IVC were presented in $\mathbf{k}$ and $\mathbf{l}\left(\mathrm{n}=5\right.$ in each group). n.s., not significant. ${ }^{* * *} P<0.001$. Scale bars: $(\mathbf{a}),(\mathbf{g})$ and $(\mathbf{j})$ are $10 \mu \mathrm{m}$, (d) is $20 \mu \mathrm{m}$. 
a

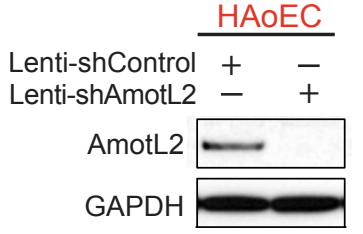

C

Static

Cellular shape

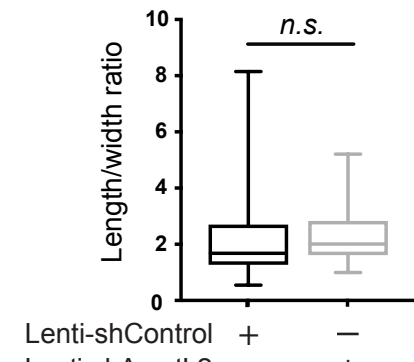

Lenti-shAmotL2 - +

d

Static Nuclear shape

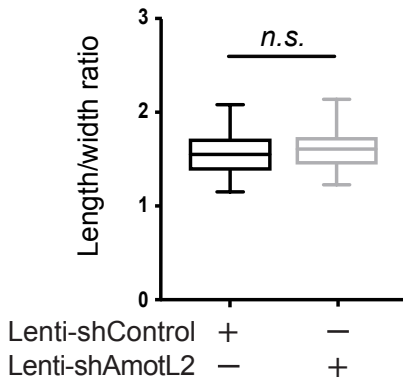

e

+Flow

Cellular shape

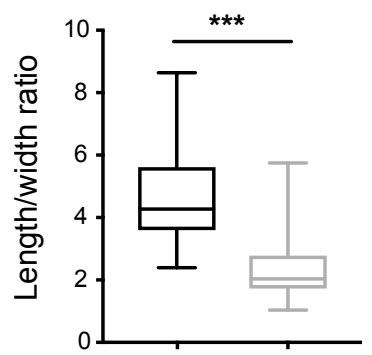

Lenti-shControl +

Lenti-shAmotL2 -

b
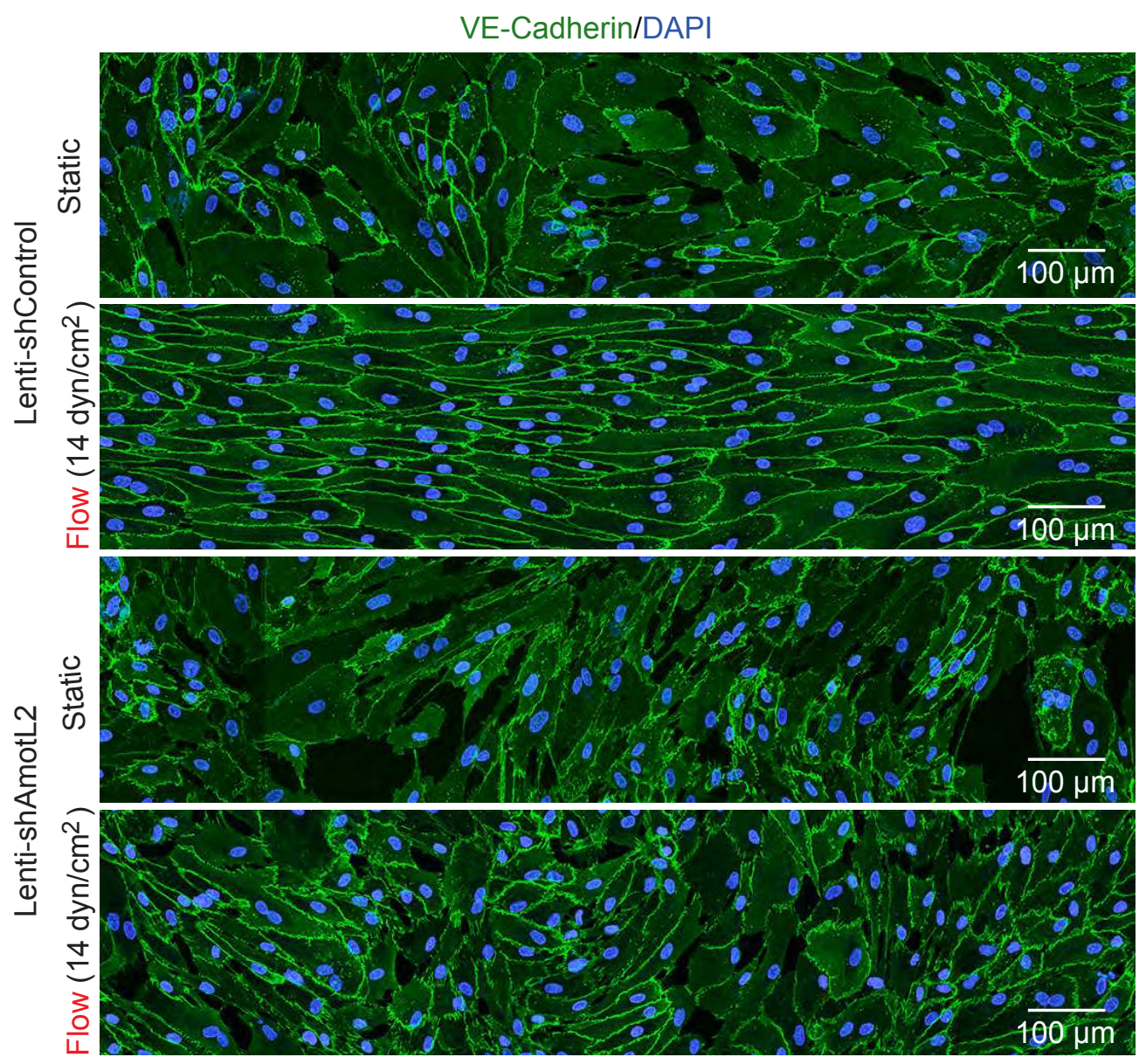

Flow $\longrightarrow$

f

+Flow

Cellular alignment

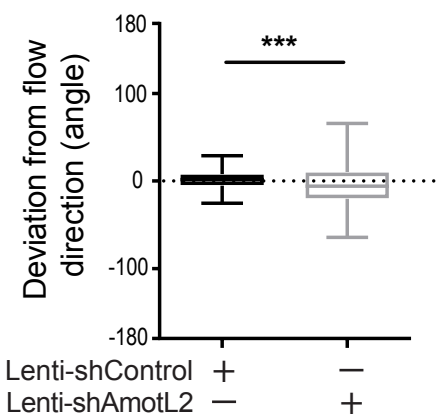

g

+Flow

Nuclear shape

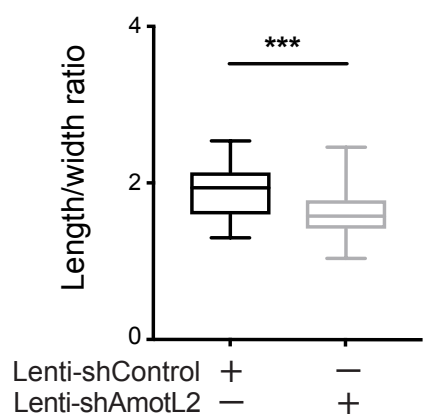

h

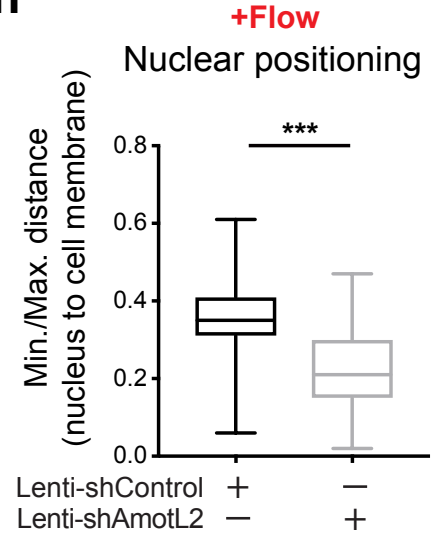


Figure 3. Effect of AmotL2 knockdown on HAoEC in vitro. a, WB analysis of whole cell lysates of confluent HAoEC cells treated with scrambled control or AmotL2 shRNA Lentivirus probed for AmotL2 and GAPDH. b, IF staining for VE-cadherin (in green) and DAPI (in blue) of HAoEC cells infected with scrambled control or AmotL2 shRNA Lentivirus under static or flow $\left(14 \mathrm{dyn} / \mathrm{cm}^{2}\right)$ conditions. Flow direction is indicated below the images. c, Quantification of cellular length/width ratio and $\mathbf{d}$, nuclear length/width ratio in scrambled control and AmotL2 shRNA Lentivirus of HAoEC cells under static conditions. Representative quantification analysis of cell length/width ratio (e, $n>80$ cells), cell alignment (f, $n>250$ cells), nuclear length/width ratio (g, $\mathrm{n}>60)$ and nuclear positioning $(\mathbf{h}, \mathrm{n}>55$ cells) were performed in both control and KD HAoEC cells. Scale bars: (b) $100 \mu \mathrm{m}$. n.s., not significant. ${ }^{* * *} P<0.001$. 
a

AmotL2 Co-IP in MS1 cells (Mass Spectrometry)

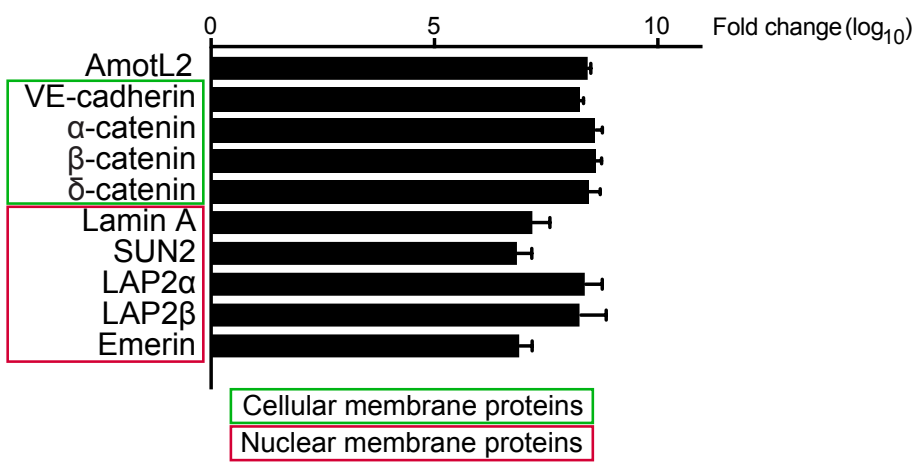

b

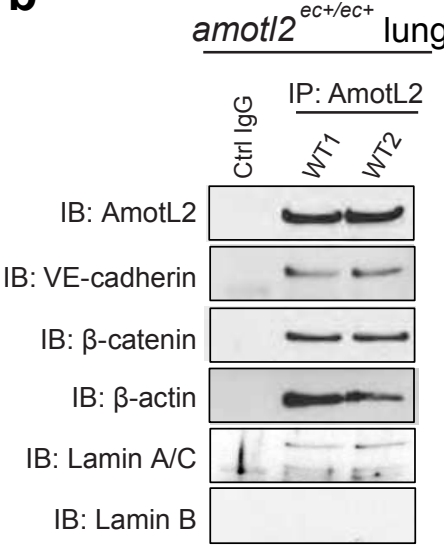

C

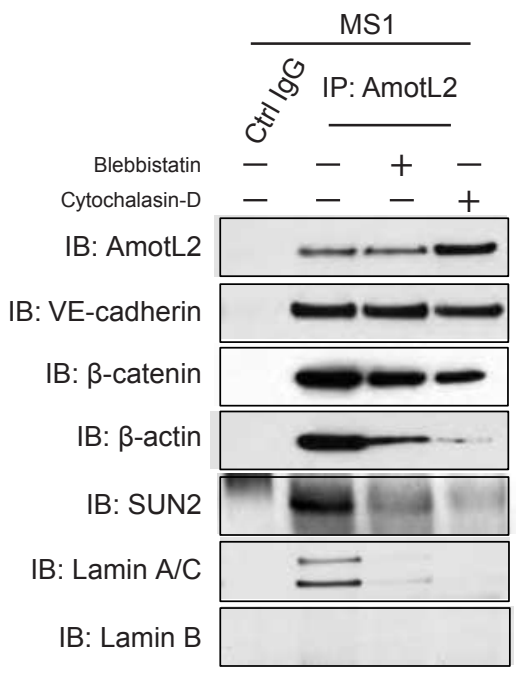

e

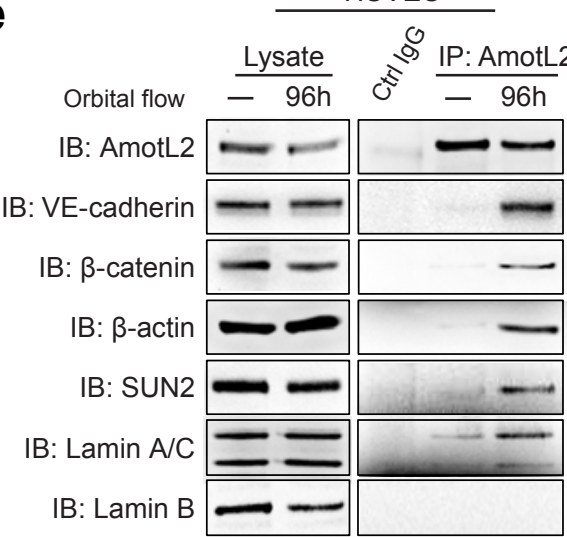

d

\section{HAoEC}

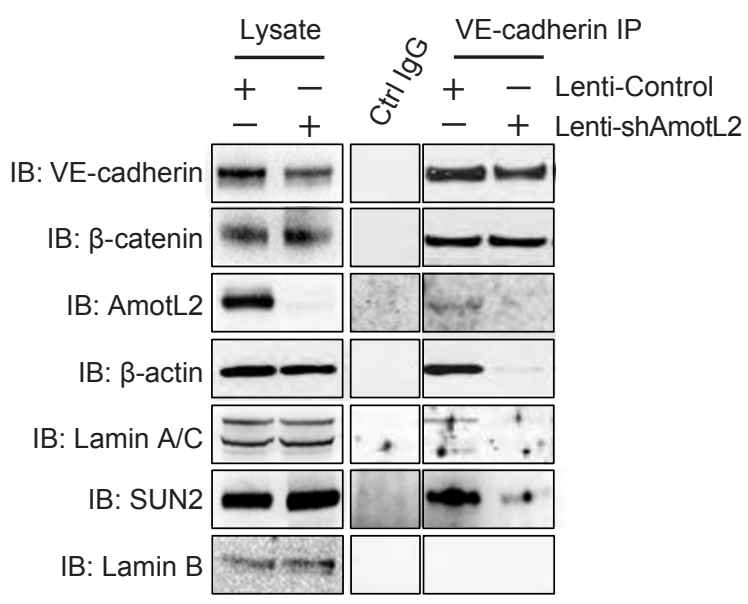

f

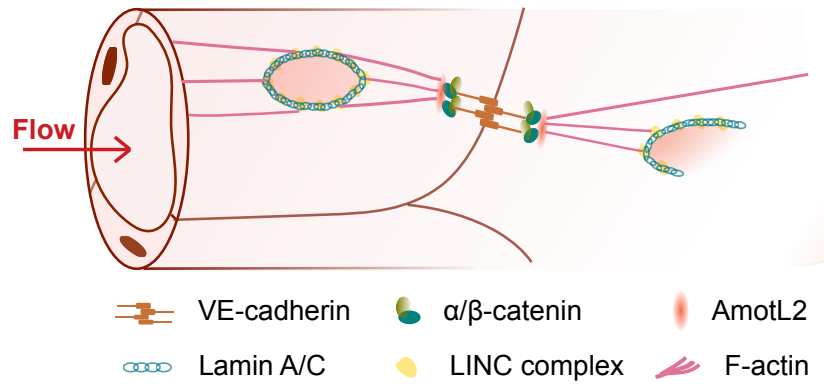




\section{Figure 4. AmotL2 links VE-cadherin to nuclear membrane protein Lamin A/C through F-}

actin. a, AmotL2, together with cellular membrane proteins (framed in green box) and nuclear membrane proteins (framed in red box) were identified from AmotL2 immunoprecipitates from MS1 cells analyzed by MS. The data was displayed with fold change $\left(\log _{10}\right)$ as compared to control immunoprecipitate samples. b, Mouse lung tissues from two randomly picked amotl $2^{\mathrm{ec}+/ \mathrm{ec}+}$ mice were lysed, subjected to IP with rabbit IgG or AmotL2 antibody and analyzed by WB. c, Murine endothelial cells (MS1) either treated with $25 \mu \mathrm{M}$ blebbistatin for $2 \mathrm{~h}$ or $1 \mu \mathrm{M}$ cytochalasin D for $1 \mathrm{~h}$. Cell immunoprecipitates with rabbit IgG or Amotl2 antibody were analyzed by WB. d, Immunoblotting (IB) of VE-cadherin associated protein complexes in HAoEC with either Lenti-Control or Lenti-shAmotL2. Whole lysate harvested from those cells were used as positive input. e, WB analysis of AmotL2 IP samples in HUVECs, either under the static condition or $96 \mathrm{~h}$ after orbital shaking $(300 \mathrm{rpm})$. Only the cells that grew at the periphery ( $6 \mathrm{~cm}$ to the edge) of a $15 \mathrm{~cm}$ dish were harvested. f, Hypothetical schematic of AmotL2 associated mechano-responsive complex forming under flow condition. 
a

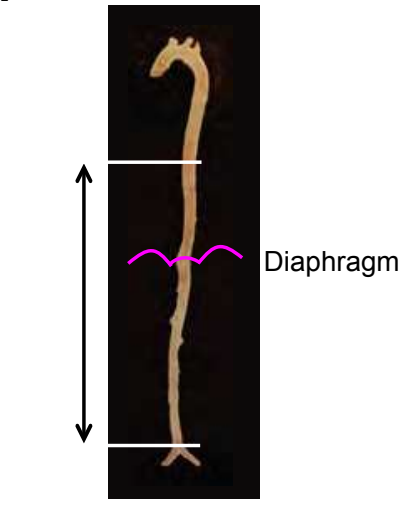

b GO terms-Biological process (amot/2 ${ }^{e c-/ e c-}$ vs amot/2 ${ }^{e c+/ e c+}$ )
C

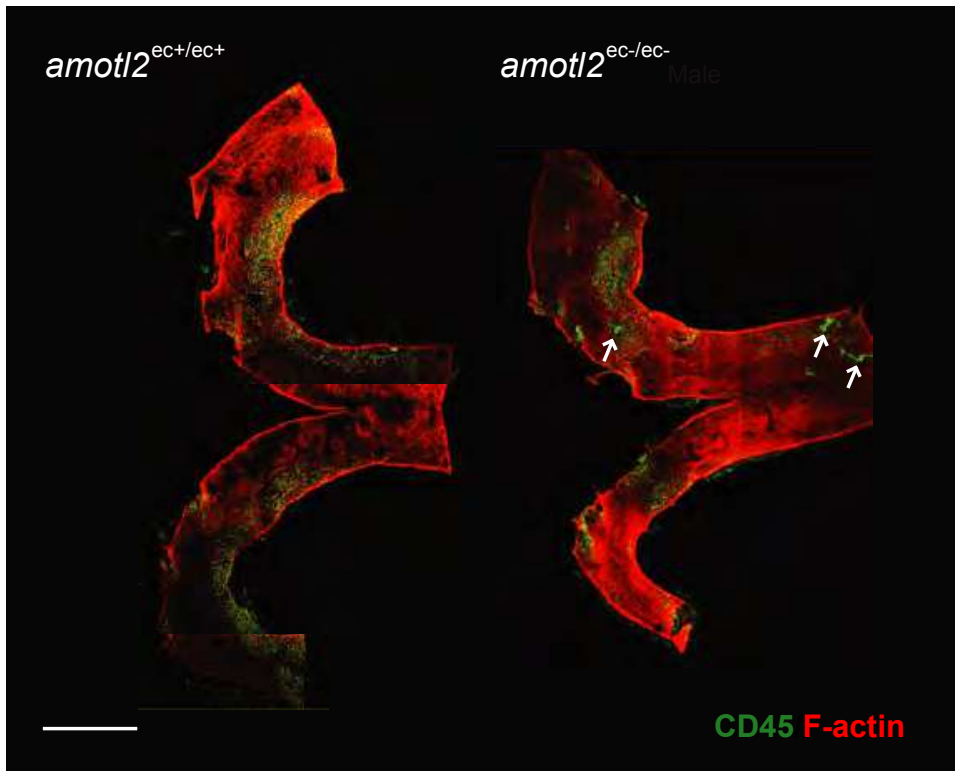

d
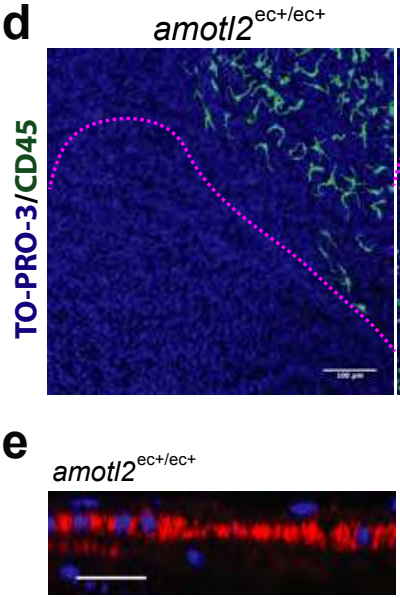
amot $12^{\mathrm{ec}-\mathrm{lec}-}$

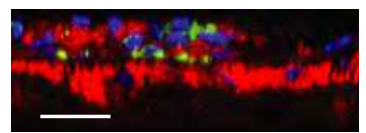

TO-PRO-3/F-actin/CD45 amot/2 $2^{\mathrm{ec}-\mathrm{ec}-}$

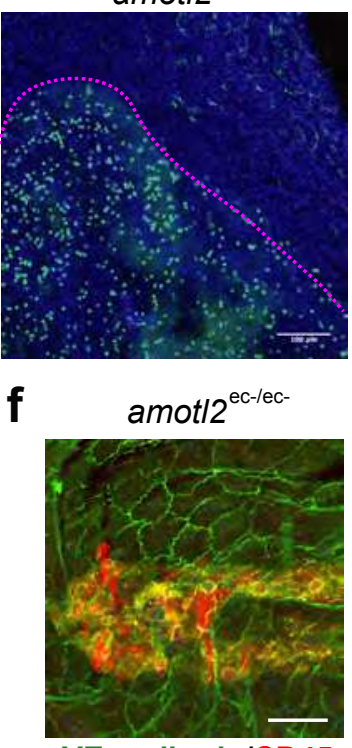

VE-cadherin/CD45 g

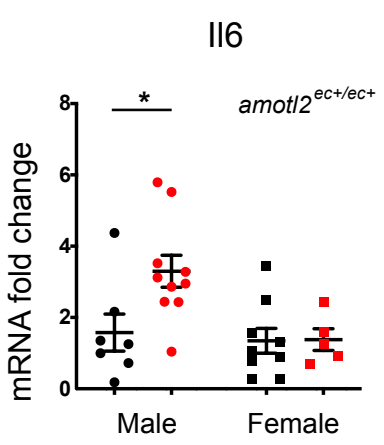

h

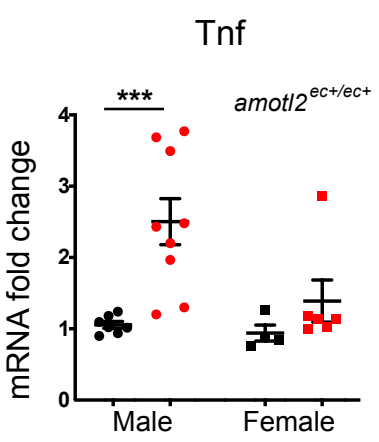

i<smiles>[10CH3]</smiles>

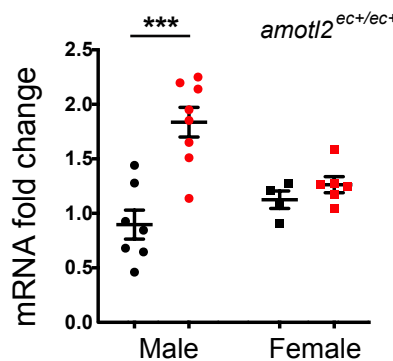

j

DA

$\mathrm{Ccl} 2$

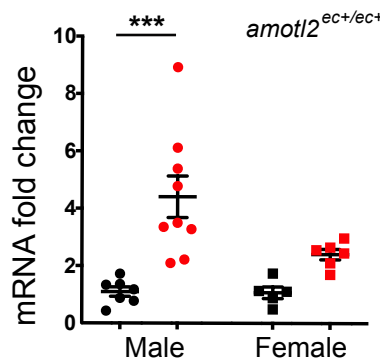


Figure 5. Absence of AmotL2 resulted in pro-inflammatory phenotype of aorta. a, Schematic of the full-length aorta. mRNA was isolated from "DA" indicating descending aorta (including part of thoracic aorta and whole abdominal aorta). b, Enriched GO terms (Biological process 2018) analyzed by Enrichr (Supplementary Table 3) and presented in the graph ranking by $-\log _{10}$ adjusted $P$ value. mRNA isolated from DAs of $\operatorname{amotl}^{\mathrm{ec}+/ \mathrm{ec}+}(\mathrm{n}=3)$ and $\operatorname{amotl}^{\mathrm{ec}-/ \mathrm{ec}-}(\mathrm{n}=5)$ mice were sent for RNA-sequencing analysis. 65 significant genes which were differentially expressed were subjected to GO terms matching. c, Whole-mount staining on aortic arch of $\mathrm{amotl}^{\mathrm{ec}+/ \mathrm{ec}^{+}}$and amotl $2^{\mathrm{ec}-\mathrm{ec}-}$ mice, stained with CD45 (in green) and phalloidin (in red). White arrows are pointing at the cluster of CD45 positive cells, which indicates the endothelial lesions. d, Representative images of CD45 positive cell clusters (in green) in amotl2 $2^{\text {ec-lec- }}$ aortic arches within the area outlined by white dash line, which were not present in the amotl2 ${ }^{\mathrm{ec}+/ \mathrm{ec}+}$ arch. e, Orthogonal view of amotl $2^{\mathrm{ec}+/ \mathrm{ec}+}$ arch and $a m o t l 2^{\mathrm{ec}-/ \mathrm{ec}-}$ lesion area stained with CD45 (in green), phalloidin (in red) and TO-PRO-3 (in blue). The luminal cells are on the top layer in the images. f, 3D-projection view of CD45+ (in red) cells invading in the endothelium (in green) in amotl2e-/ec- $\operatorname{arch}$. The image was processed in ImageJ. g-j, The relative mRNA expression levels of $I l 6, \operatorname{Tnf}, \mathrm{Cd} 68$, and Ccl2 were determined with TaqMan probes. The mRNA was isolated from DA (g-j) tissues of 13 amotl $2^{\mathrm{ec}+/ \mathrm{ec}+}$ mice (in black dots, male $\mathrm{n}=7$, female $\mathrm{n}=4-9$ ) and 15 amotl $2^{\mathrm{ec}-/ \mathrm{ec}-}$ mice (in red dots, male $n=8-10$ and female $n=5-6$ ). Relative expression levels were normalized versus Hprt. Foldchanges are quantified, and data shown are mean \pm S.E. Scale bars: (c) $1000 \mu \mathrm{m}$, (d) $100 \mu \mathrm{m}$, and (e) $20 \mu \mathrm{m} .{ }^{*} P<0.05 .{ }^{* * *} P<0.001$. 
a

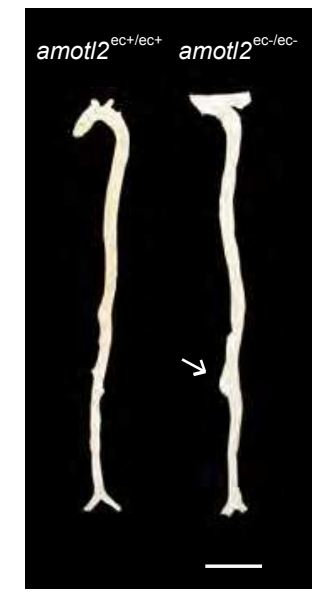

C

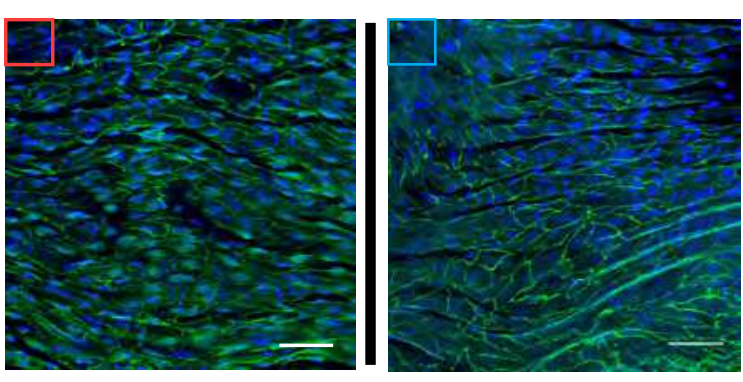

d

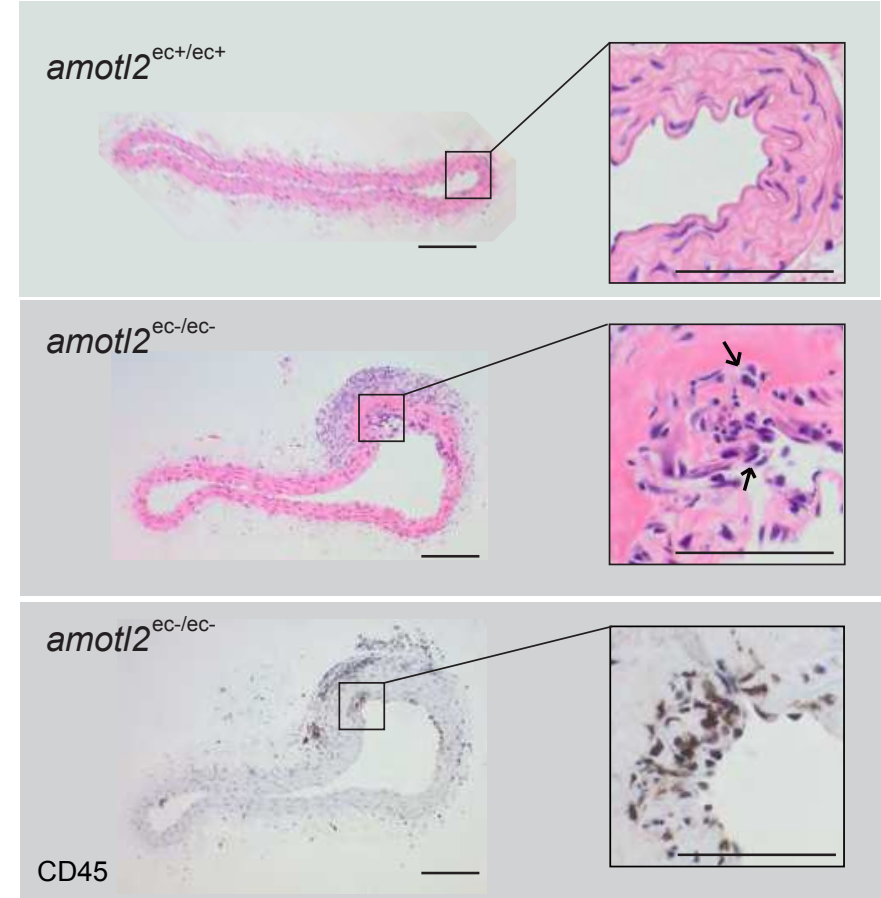

b
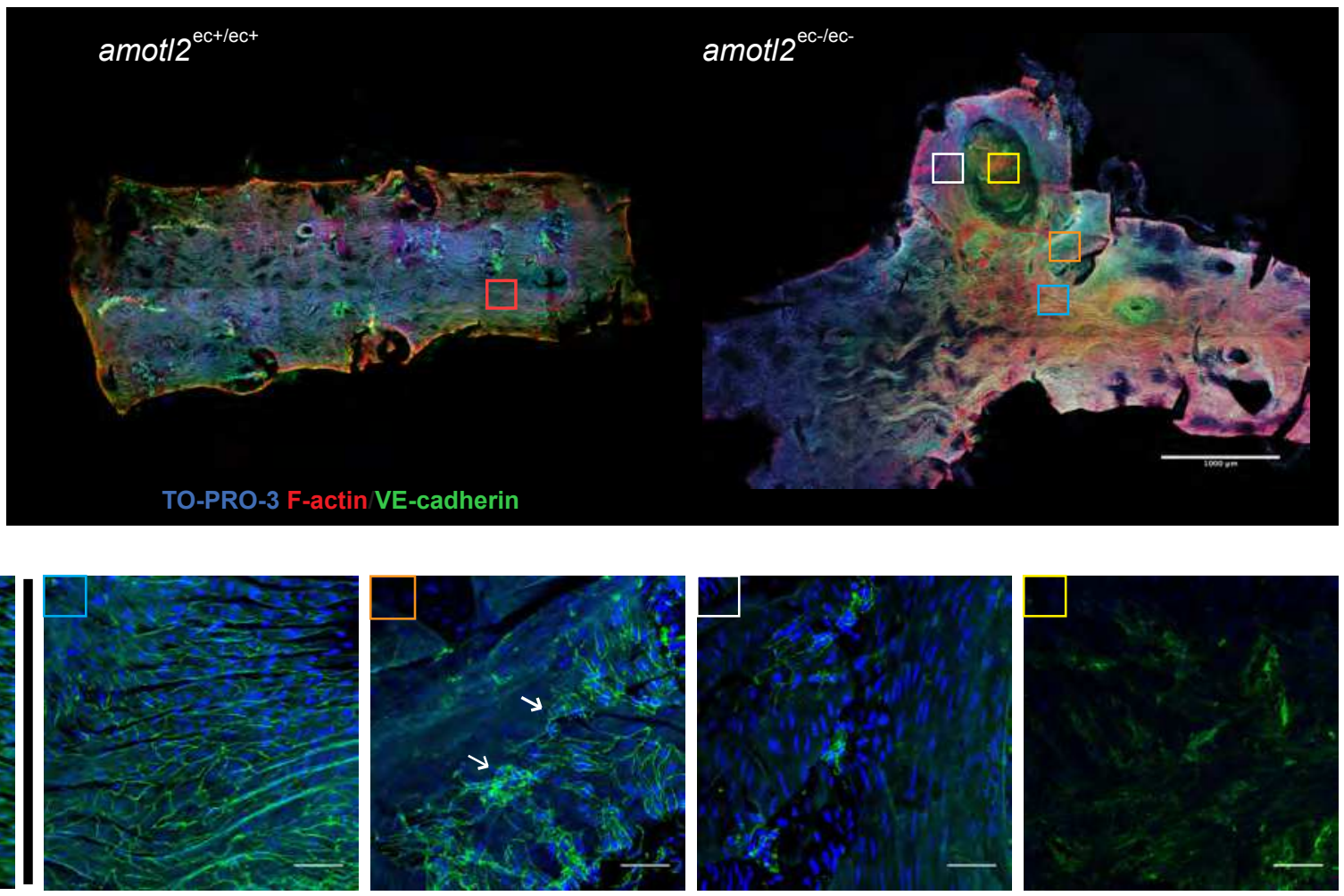

e

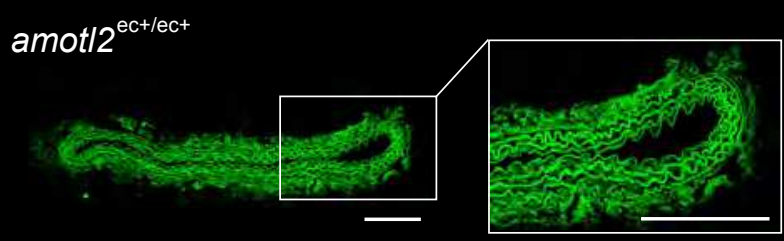

amot/2 ${ }^{\mathrm{ec}-\mathrm{lec}-}$

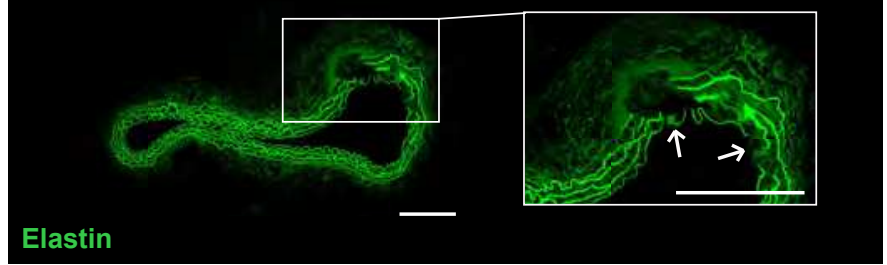


Figure 6. Aortic aneurysms were induced in male amotl ${ }^{\text {ec-/ec- }}$ mice. a, The dissected full-length aorta from amotl $2^{\mathrm{ec}+/ \mathrm{ec}+}$ and amotl2 $2^{\mathrm{ec}-/ \mathrm{ec}-}$ mice. White arrow indicates the abdominal aortic aneurysm (AAA), which occurred close to the renal artery bifurcations. b, Representative images

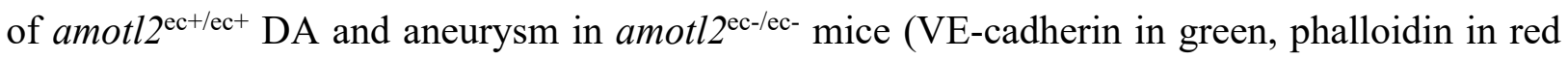
and TO-PRO-3 in blue). Selective area (boxed) was magnified and presented in c. The red box shows wild-type EC morphology in amotl $2^{\mathrm{ec}+/ \mathrm{ec}+}$ DA, while the blue box, orange box, white box, and yellow box sequentially outlined in aneurysm reveal the impact on endothelium during aneurysm formation. In orange box, endothelium was damaged and regressed, indicated by white arrows. The DAs from amotl $2^{\mathrm{ec}+/ \mathrm{ec}+}$ and amotl2 $2^{\mathrm{ec}-/ \mathrm{ec}-}$ mice with aneurysm were fixed and embedded by paraffin. H\&E (upper and middle panel) and CD45 (lower panel) staining was applied on the paraffin sections in d. Additionally, aneurysm section was stained by CD45 and showed in the bottom panel. Boxed areas are magnified and placed on the right. In the aneurysm section, abnormal nuclei penetrated were observed along the lumen side indicated by black arrows. e, Elastin structure visualized by auto-fluorescence captured under UV light. Layers of elastin were presented in magnified images in white boxes on the right. White arrows indicate normal elastin structure in amotl $^{\mathrm{ec}+/ \mathrm{ec}+}$ DA and discontinuation in amotl $2^{\mathrm{ec}-/ \mathrm{ec}-}$ aneurysm, respectively. Scale bars: $1000 \mu \mathrm{m}(\mathbf{a}), 25 \mu \mathrm{m}(\mathbf{c})$ and $100 \mu \mathrm{m}(\mathbf{d}$ and $\mathbf{e})$. 


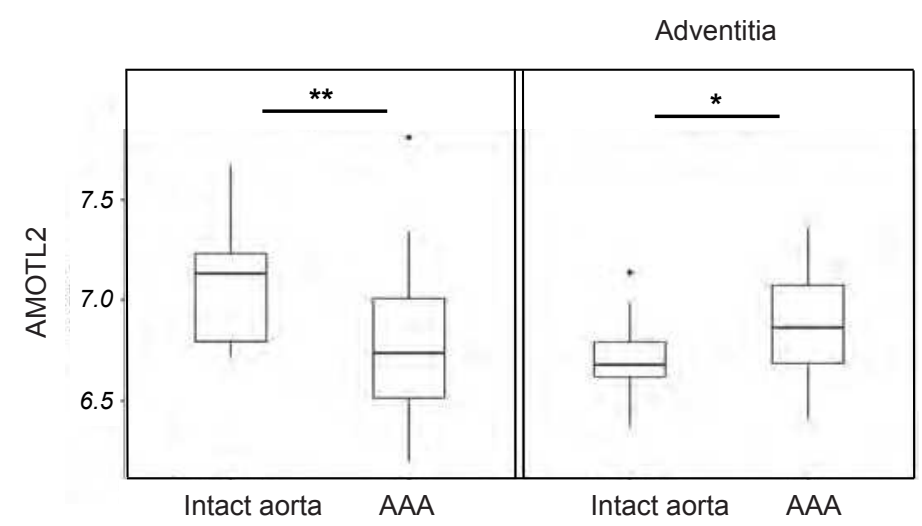

C
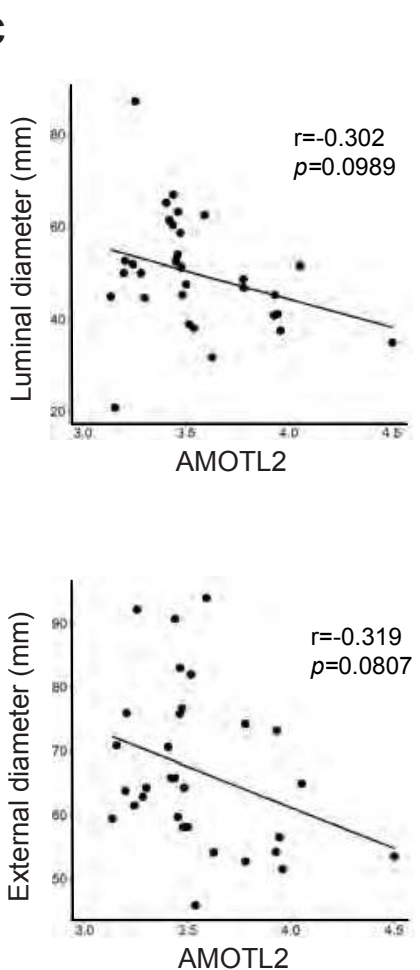

d

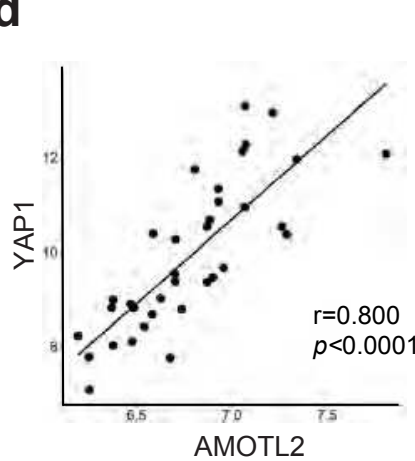

Adventitia

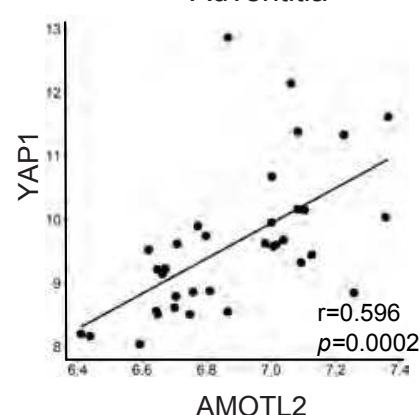

e

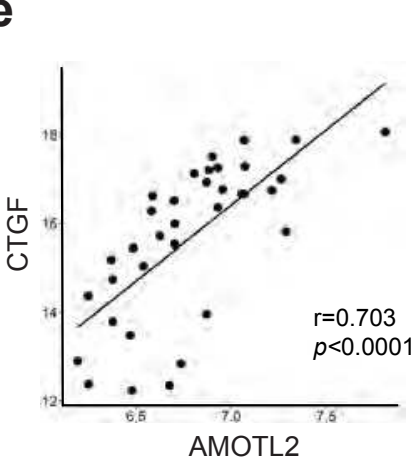

Adventitia

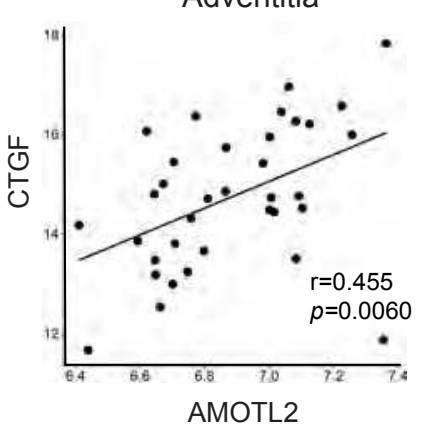

f

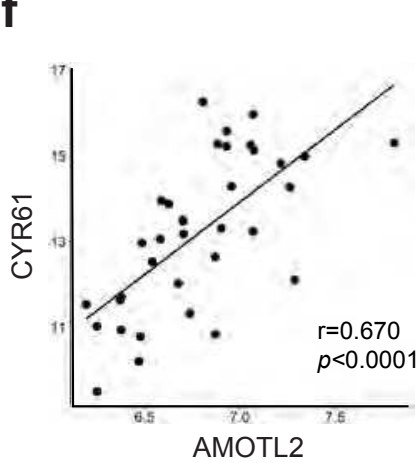

Adventitia

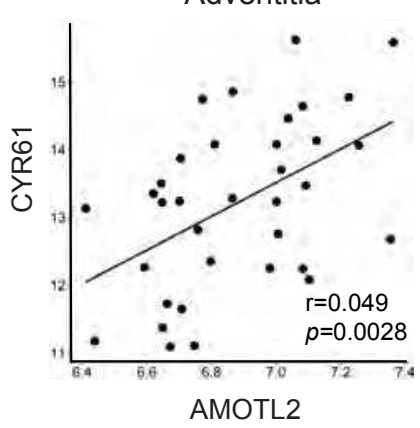

g

Hypothetical schematic of aneurysm formation

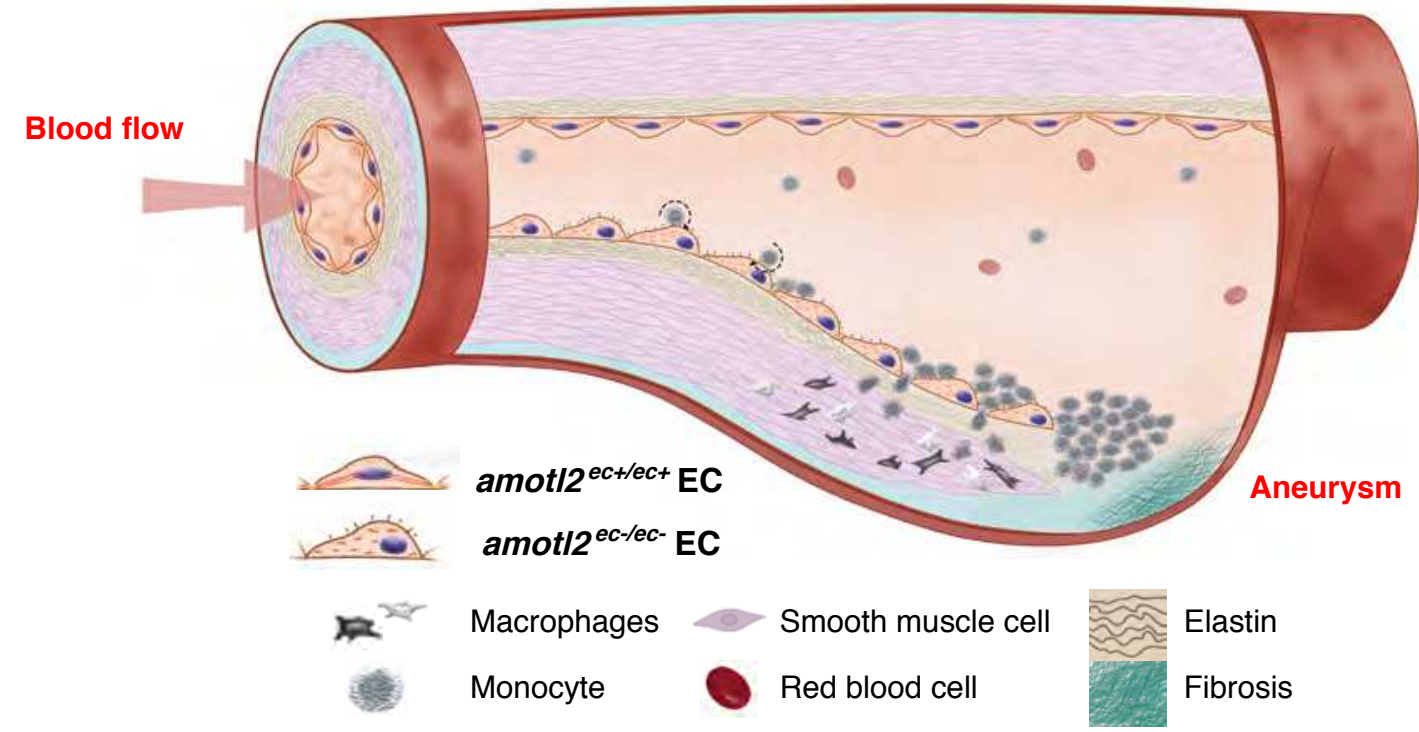


Figure 7. YAP1 and AMOTL2 correlates in human AAA patients. AAA samples of intima/media region of aortic wall not covered by an intraluminal thrombus i.e., aneurysm wall covered by an EC layer were obtained at open AAA surgery, and mRNA were isolated from both media and adventitia. a, Schematic depicting anatomical view of the media and adventitia tissue in the aorta. b, Quantification of AmotL2 mRNA expression in medial and adventitial tissues from both intact aortae (13 healthy donors) and dilated aortae (35 AAA patients). ${ }^{*} P<0.05$. ${ }^{* *} P<0.01$. c, The correlations between AMOTL2 mRNA level of expression and the luminal (upper panel) / external (lower panel) diameter of aneurysms in AAA patients $(\mathrm{n}=35)$. AMOTL2 expression level was based on the expression of the first exon from 3' end, detected by specific exon probe, which represents the full-length isoform of AMOTL2. AMOTL2 correlation with YAP1, CTGF and CYR61 of mRNA level of expression were shown in d-f, respectively. AMOTL2 expression level was calculated with mean value of every exon expression detected. For each correlation analysis, samples from media were present on top while samples from adventitia on bottom. Correlation coefficient $r$ and $p$-value are labelled in each individual figure. 21 male and 10 female patients were analyzed in $\mathbf{c}$, while 25 male and 10 female patients were enrolled in d-f. g, Hypothetical schematic of the formation of an AAA. The step-by-step formation of an AAA includes EC activation, immune cell rolling and attachment, leukocyte extravasation, macrophage differentiation, thrombus formation, and finally, lumen dilation (aneurysm). 

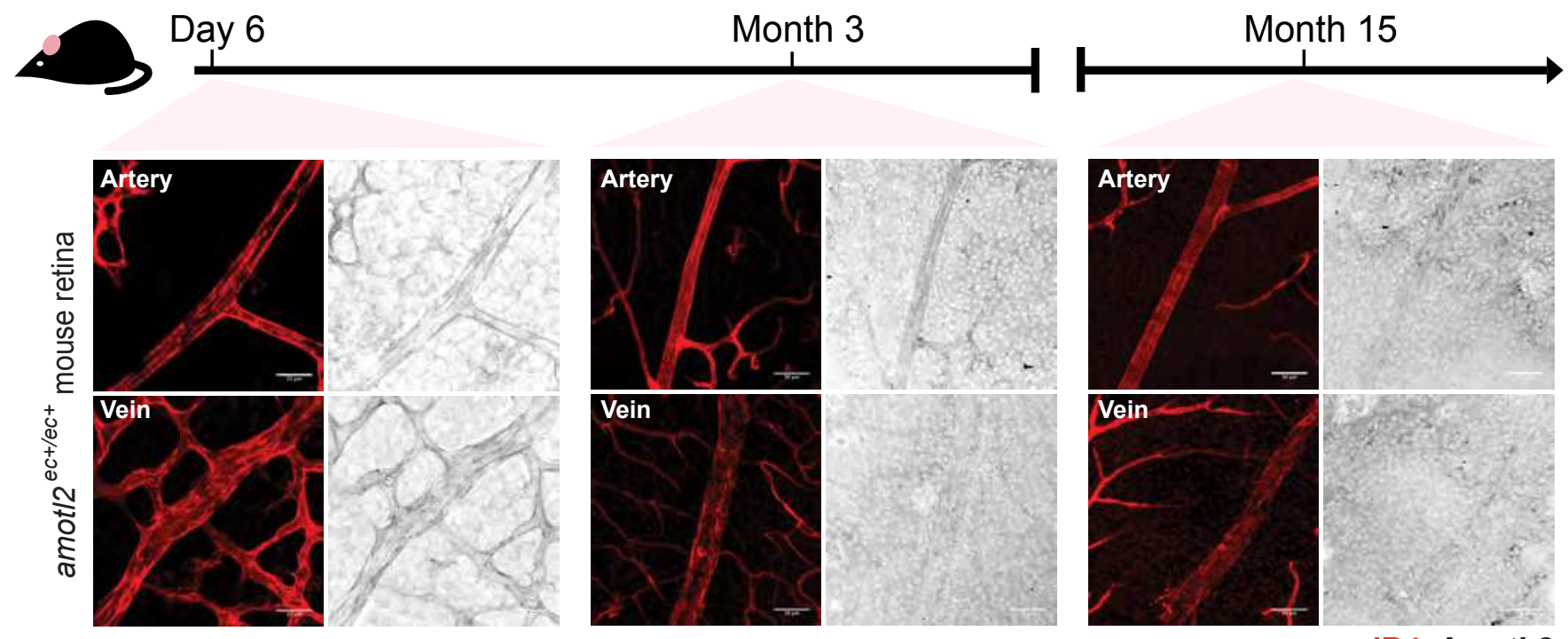

IB4 AmotL2 
Supplementary Figure 1. AmotL2 expression pattern in mouse retina of different ages. amotl $2^{\mathrm{ec}+/ \mathrm{ec}^{+}}$mice retinas were stained with IB4 (in red) and AmotL2 (in gray) at the age of postnatal day 6, month 3 and month 15, which are placed in the left, middle and right panel, respectively. Both representative images of vasculature in arteries and veins are shown for each time point. Scale bars: $25 \mu \mathrm{m}$ (left panel) and $50 \mu \mathrm{m}$ (middle/right panel). 
a

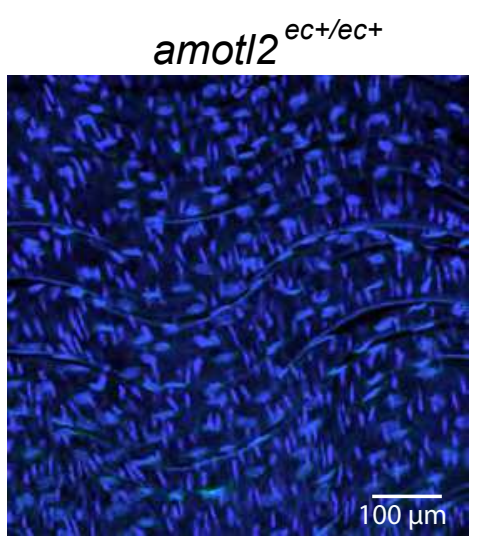

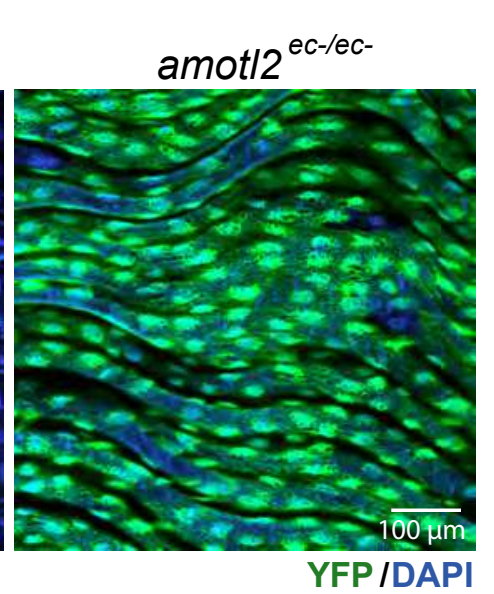

b

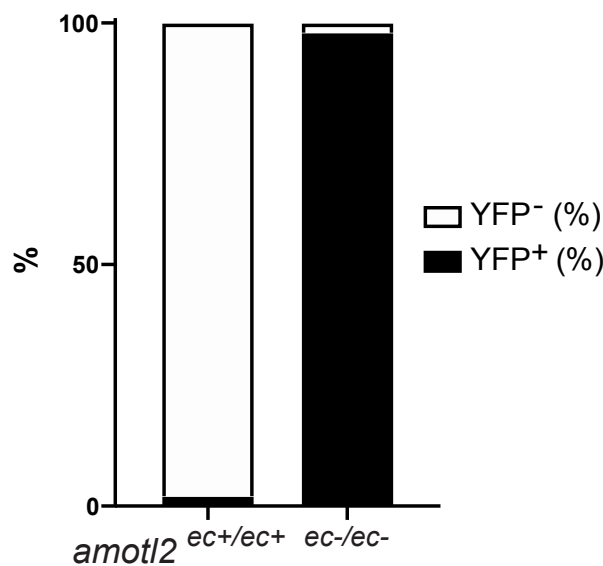


Supplementary Figure 2. Recombination efficiency in amotl2 $\left.2^{\text {flox } f l o x} \operatorname{Cdh5(PAC)}\right)^{\text {CreERT2 }}$ ROSA26-EYFP mice. a, GFP immunofluorescence staining (in green) was performed to visualize the YFP reporter, as a marker for Cre-recombinase expression of DAs in $a m o t l 2^{\mathrm{ec}+/ \mathrm{ec}+}$ and amotl2 ${ }^{\text {ec-lec- }}$ Cdh5(PAC) ${ }^{\text {CreERT2 }}$ ROSA26-EYFP mice. b, Quantification of the percentage $\mathrm{YFP}^{+} / \mathrm{YFP}^{-}$of 3 representative amotl $2^{\mathrm{ec}+/ \mathrm{ec}+}$ and amotl2 $^{\mathrm{ec}-/ \mathrm{ec}-}$ DAs. Size bars: $100 \mu \mathrm{m}$. 
a

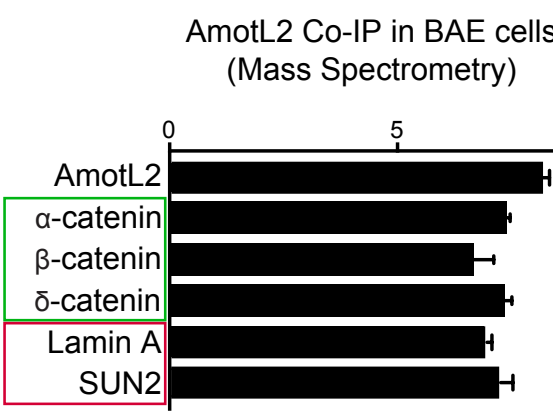

Cellular membrane proteins

Nuclear membrane proteins b

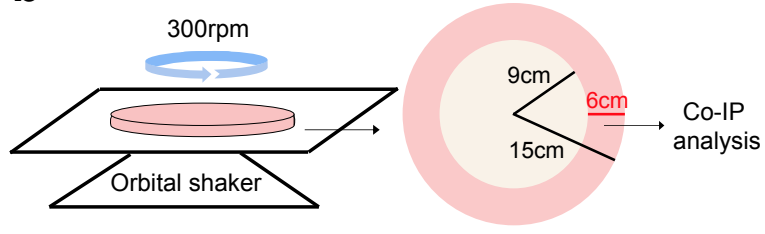

C

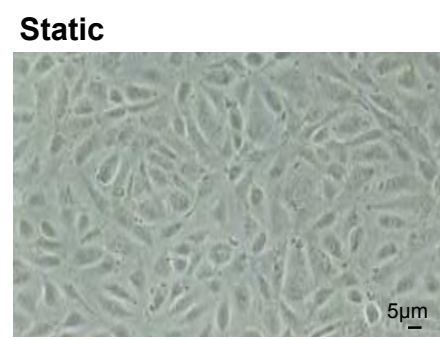

Flow 96h

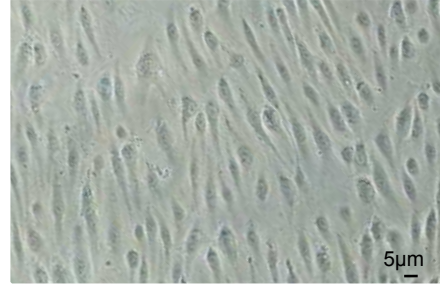

Cell orientation angle

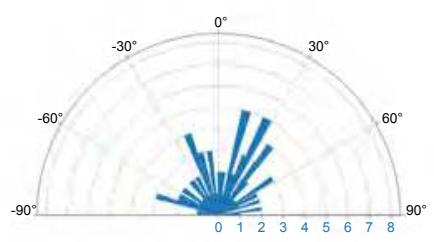

Count

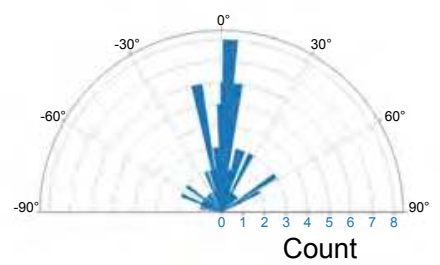




\section{Supplementary Figure 3. AmotL2 links VE-cadherin to LINC complex through actin. a,}

Catenins in cellular membrane proteins (framed in green box) and nuclear membrane proteins (framed in red box) were identified from AmotL2 immunoprecipitates from BAE cells analyzed by MS. The data was displayed with fold change $\left(\log _{10}\right)$ as compared to control IP samples. b, Schematic describing how circulatory flow was applied to HUVECs cultured in $15 \mathrm{~cm}$ dish (300rpm, 96h). The peripheral area with a width of $6 \mathrm{~cm}$ (pink area) is where the cells were harvested for CO-IP experiments. c, Bright field images of HUVECs located in the pink area indicated in $\mathbf{b}$, illustrating the cell morphology in both static and one post-flow conditions (96h on orbital shaker). Polar bar chart depicting the angle of cell orientation are presented on the right side. The mean of the actual angular value was normalized to $0^{\circ}$ in both conditions. Size bars: 5 $\mu \mathrm{m}$. 


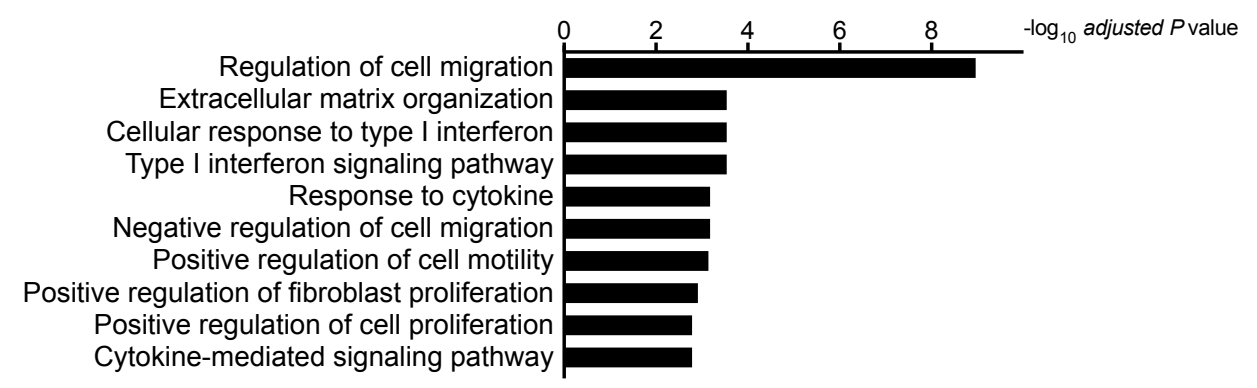

b

Vcam-1

C

Icam-1

d

Cxcl10

e Ccl5

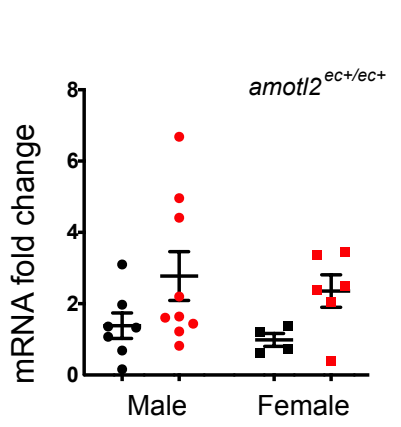

f

DA

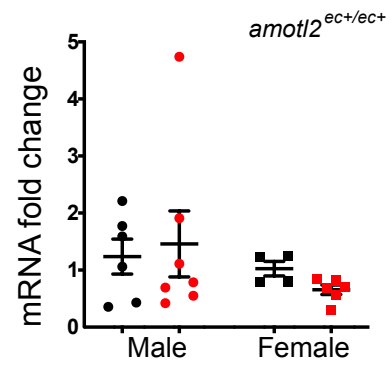

DA

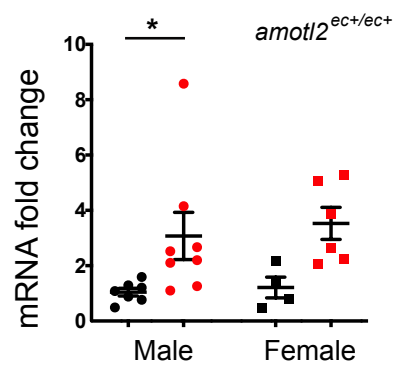

h

Cd8
DA

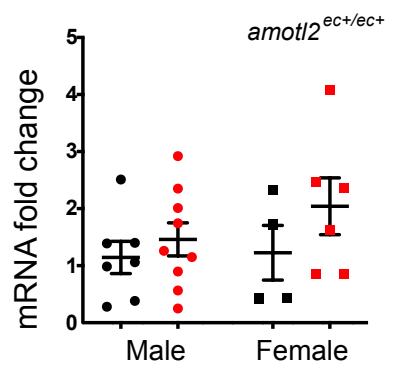

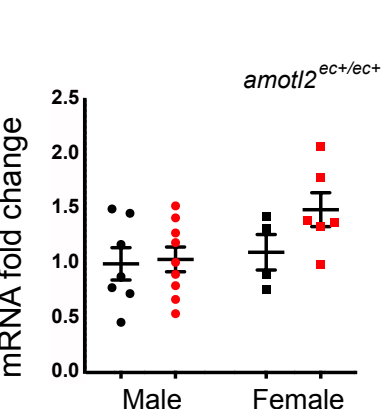

g
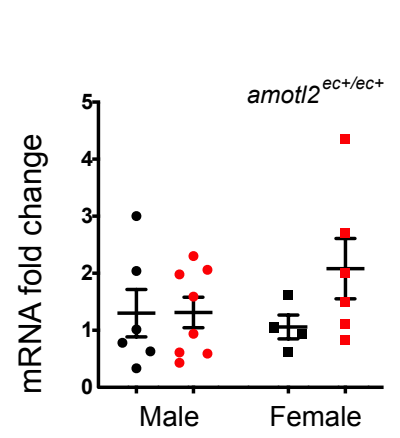
Supplementary Figure 4. Absence of AmotL2 provokes immune response in the aorta. a, Enriched GO terms (Biological process 2018) analyzed by Enrichr (Supplementary Table 4) and presented in the graph ranking by $-\log _{10}$ adjusted $P$ value. mRNA isolated from MS1 cells treated by control or AmotL2 siRNA ( $\mathrm{n}=3)$ mice were sent for RNA-sequencing analysis. Top 500 differentially expressed genes with the lowest adjusted-P value were subjected to GO terms matching. b-h, mRNA was isolated separately from descending aorta (DA, in red), and analyzed by TaqMan qRT-PCR. Relative expression levels of Vcam-1 (b), Icam-1 (c), Cxcl10 (d), Ccl5 (e), $C d 4$ (f), $C d 8$ (g), and $C d 19$ (h) were normalized versus Hprt. mRNA was obtained from about 10 amotl $2^{\mathrm{ec}+/ \mathrm{ec}+}$ mice (in black dots, male $\mathrm{n}=5-7$, female $\mathrm{n}=4-5$ ) and 14 amotl $2^{\mathrm{ec}-\mathrm{ec}-}$ mice (in red dots, male $n=7-9$ and female $n=6$ ). Fold-changes are quantified, and data shown are mean \pm S.E. ${ }^{*} P<0.05$. ${ }^{* * *} P<0.001$. To note that, all the $p$-values unlabelled in the graphs indicate "not statistically significant”. 
a

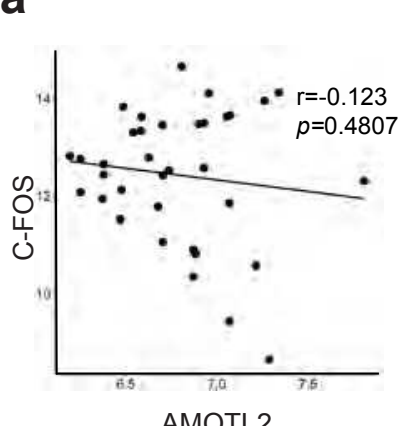

AMOTL2

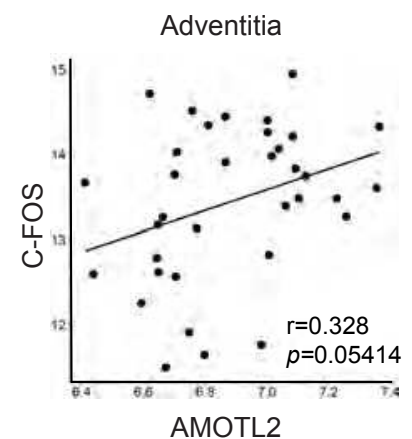

b

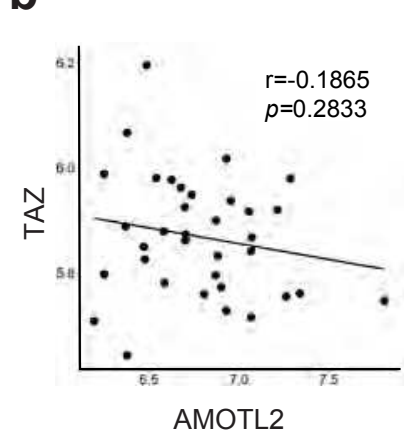

Adventitia

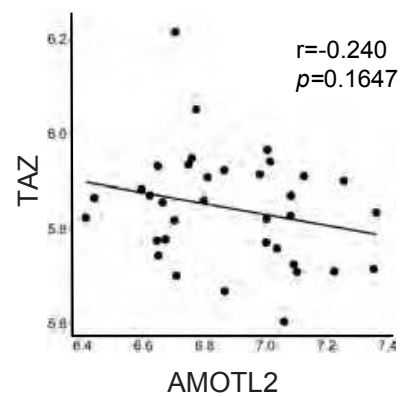

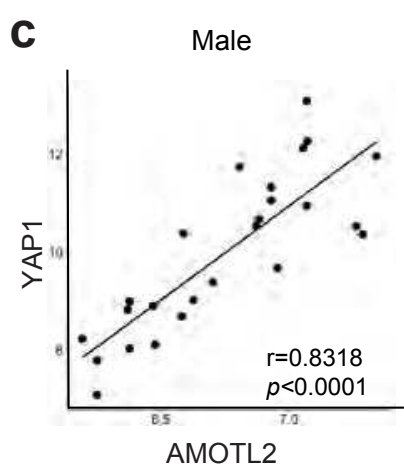
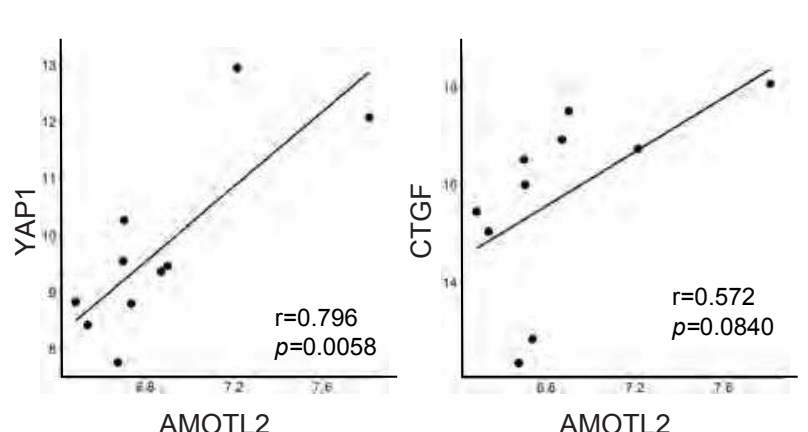
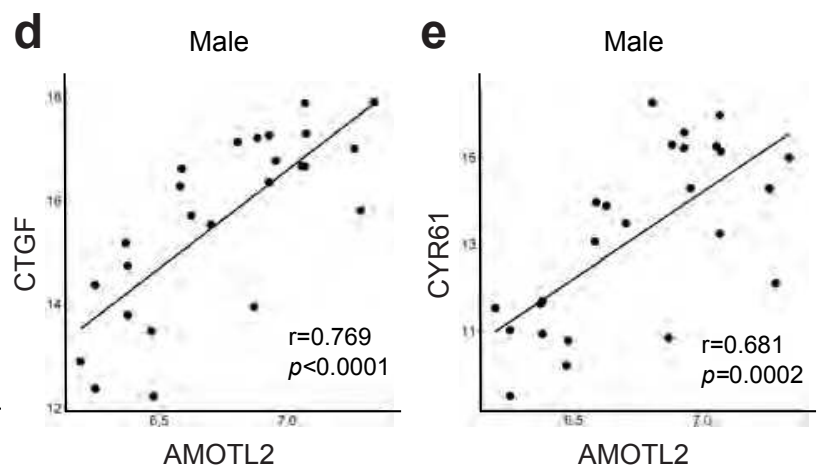

f

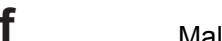

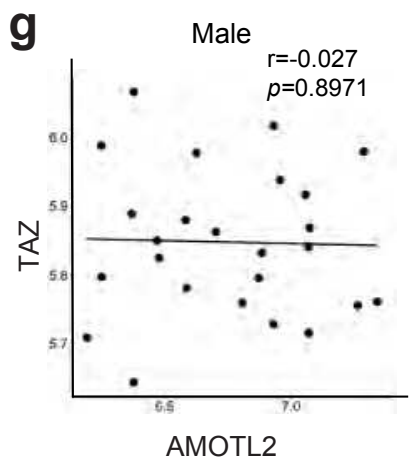
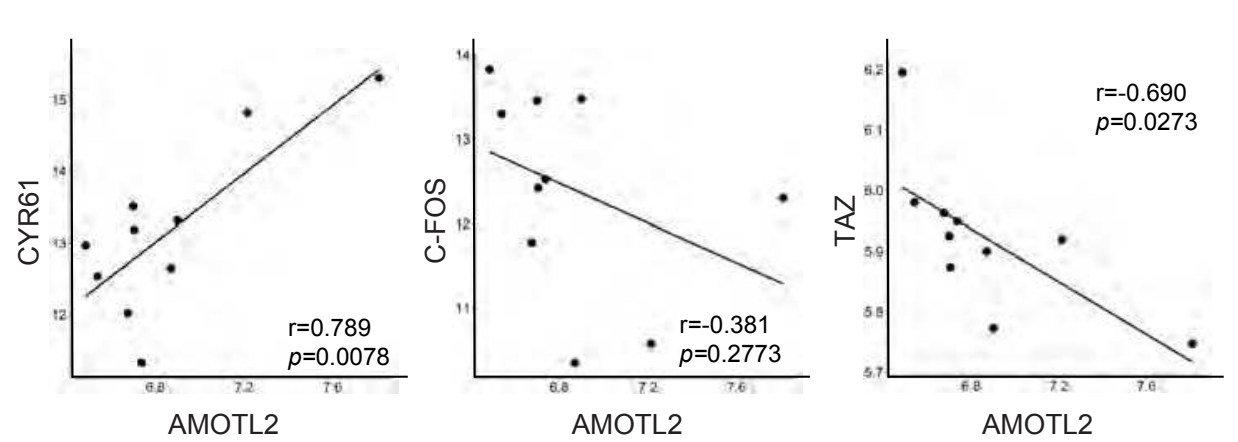
Supplementary Figure 5. The AmotL2 correlation with Hippo pathway in AAA samples from human patients. mRNA expression level correlations of AmotL2 with C-FOS in a, and TAZ in b were analyzed separately in media (left panel) and adventitia (right panel) tissues. 25 male and 10 female patients were enrolled. Aortic media tissues from male and female of AAA patients were analyzed separately for AmotL2 mRNA expression correlation with YAP1 (c), CTGF (d), CYR61 (e), C-FOS (f) and TAZ (g). Correlation coefficient $r$ and $p$-value are labelled in each individual figure. 


\section{Figure 3a}
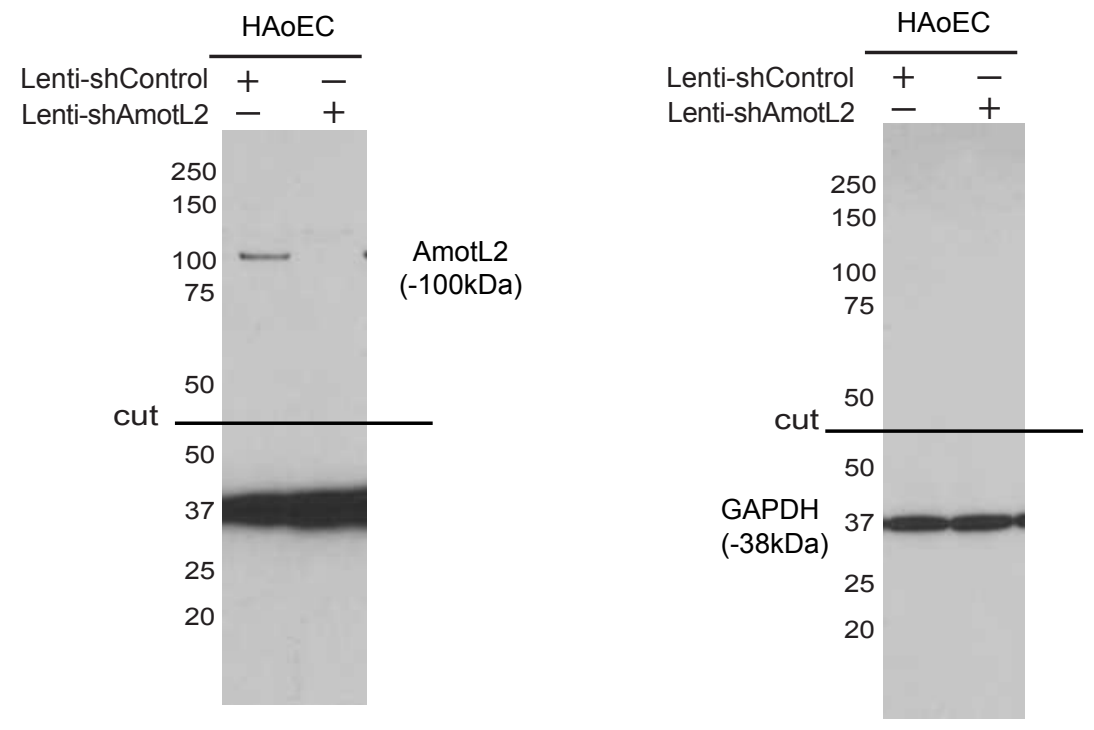

Figure 4b
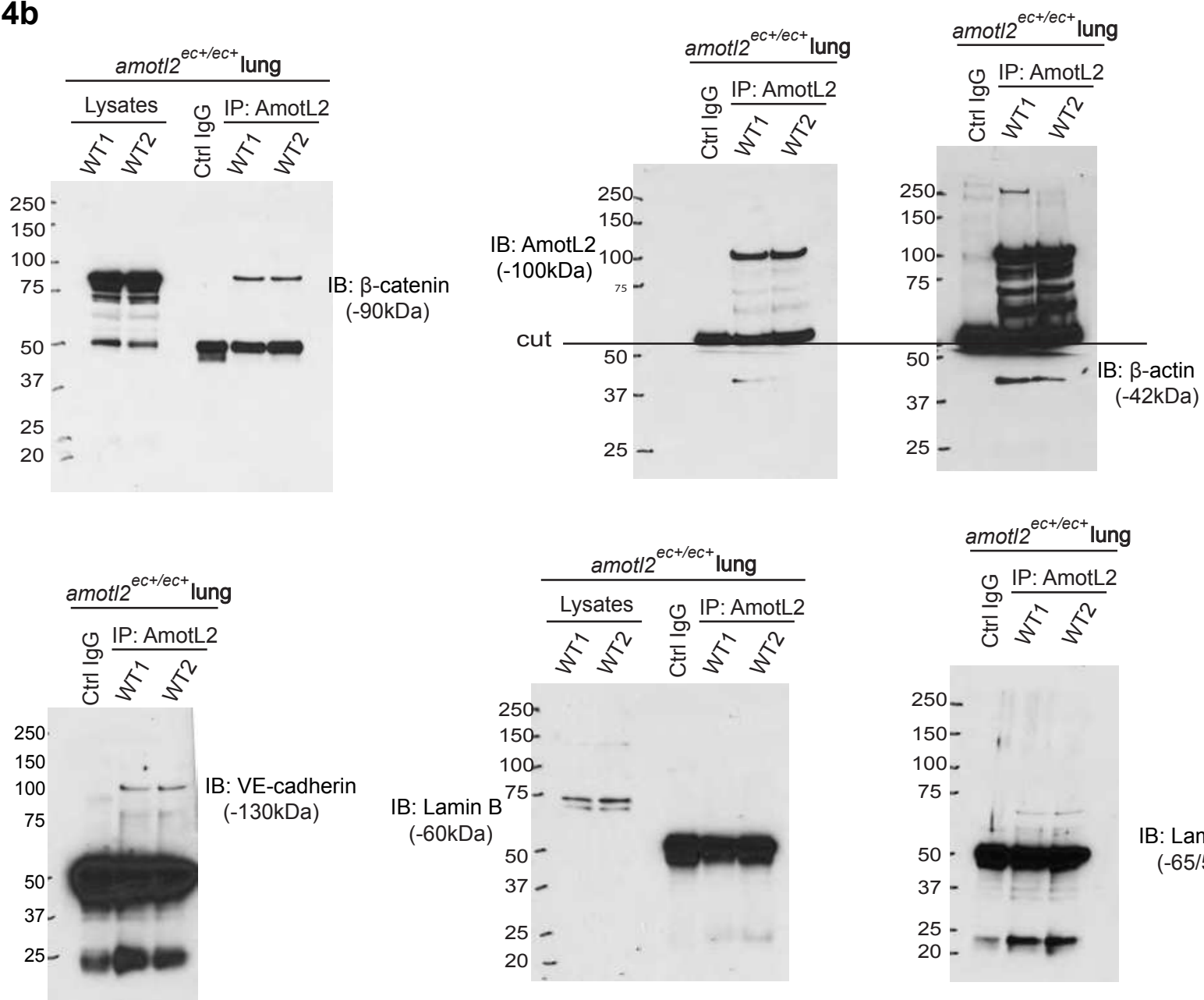

$\underline{\text { amot/2 }}$ ect/ec+ $^{\text {lung }}$

(I): AmotL2

峑 5 今

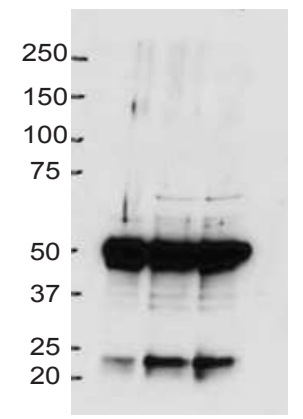

IB: Lamin A/C $(-65 / 55 \mathrm{kDa})$ 


\section{Figure 4c}

All in MS1 cells
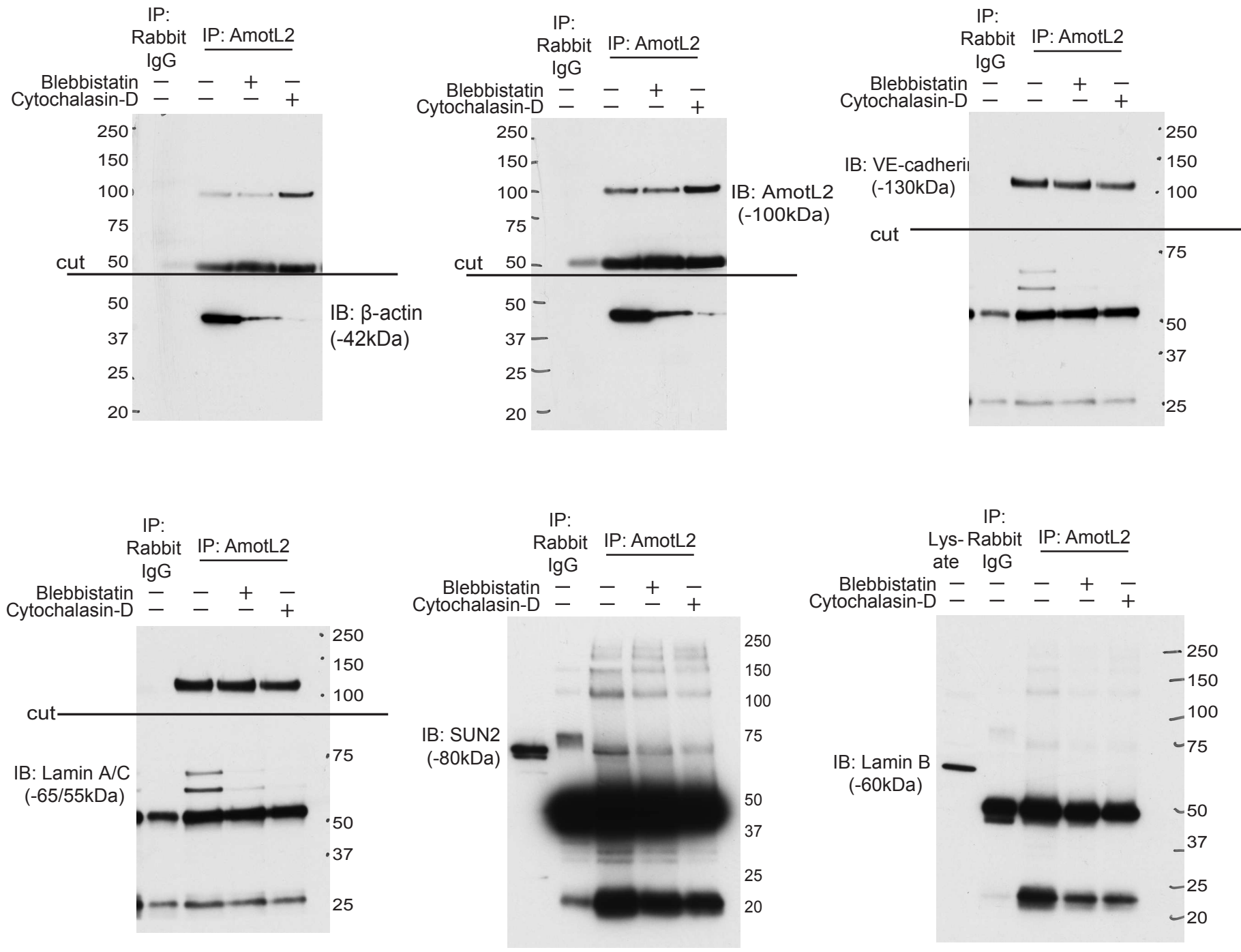

IP:

Rabbit IP: AmotL2

$\lg G$

$\underset{\text { Blebbistatin }}{\operatorname{C}} \quad \pm \quad+\quad$

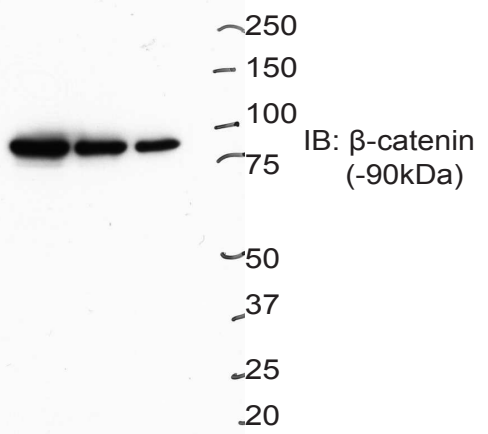


Figure 4d

All in HAoEC
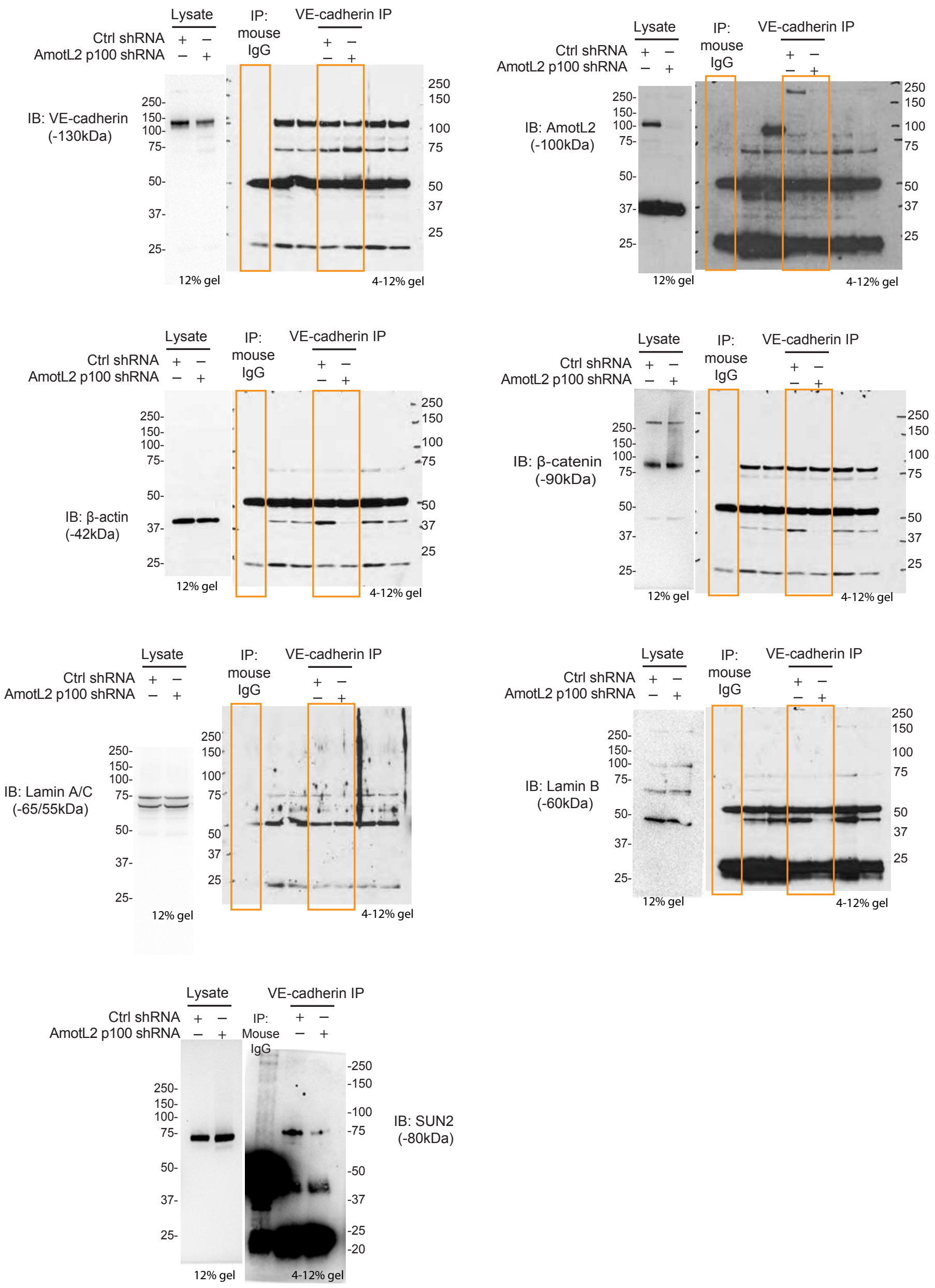

Supplementary Figure 8. Full length blots to Figure 4d. 


\section{Figure 4e}

All in HUVEC
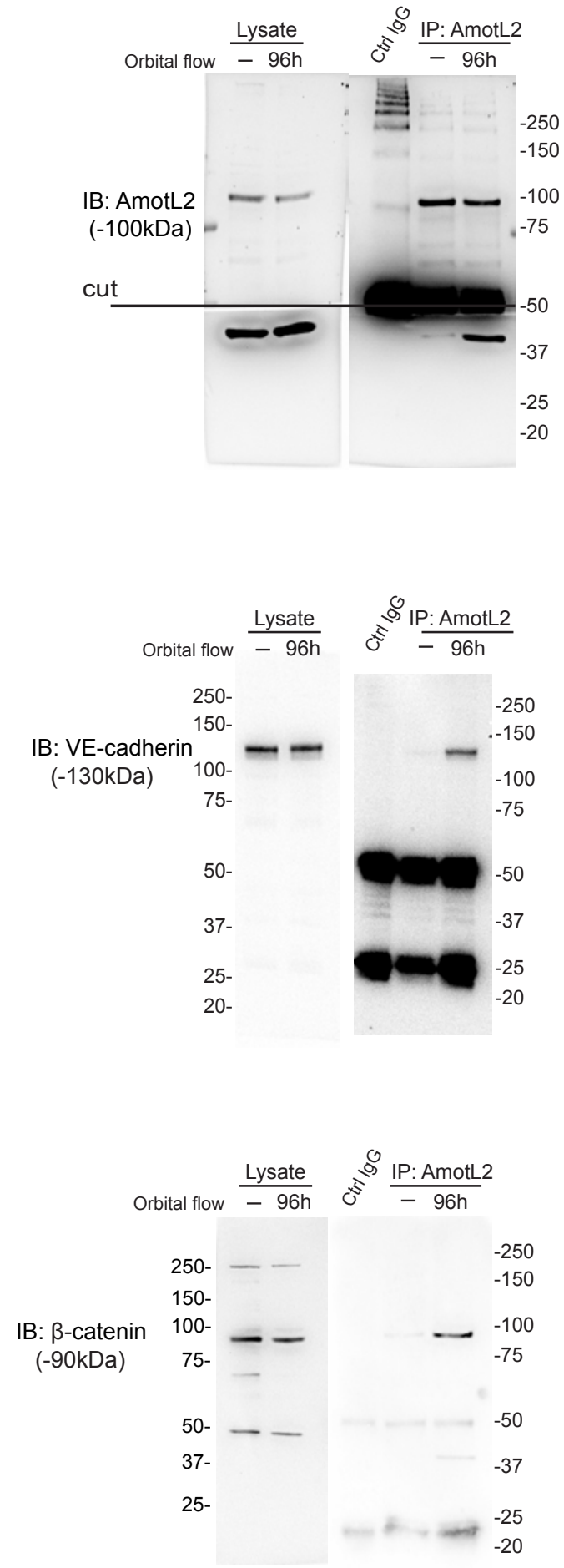
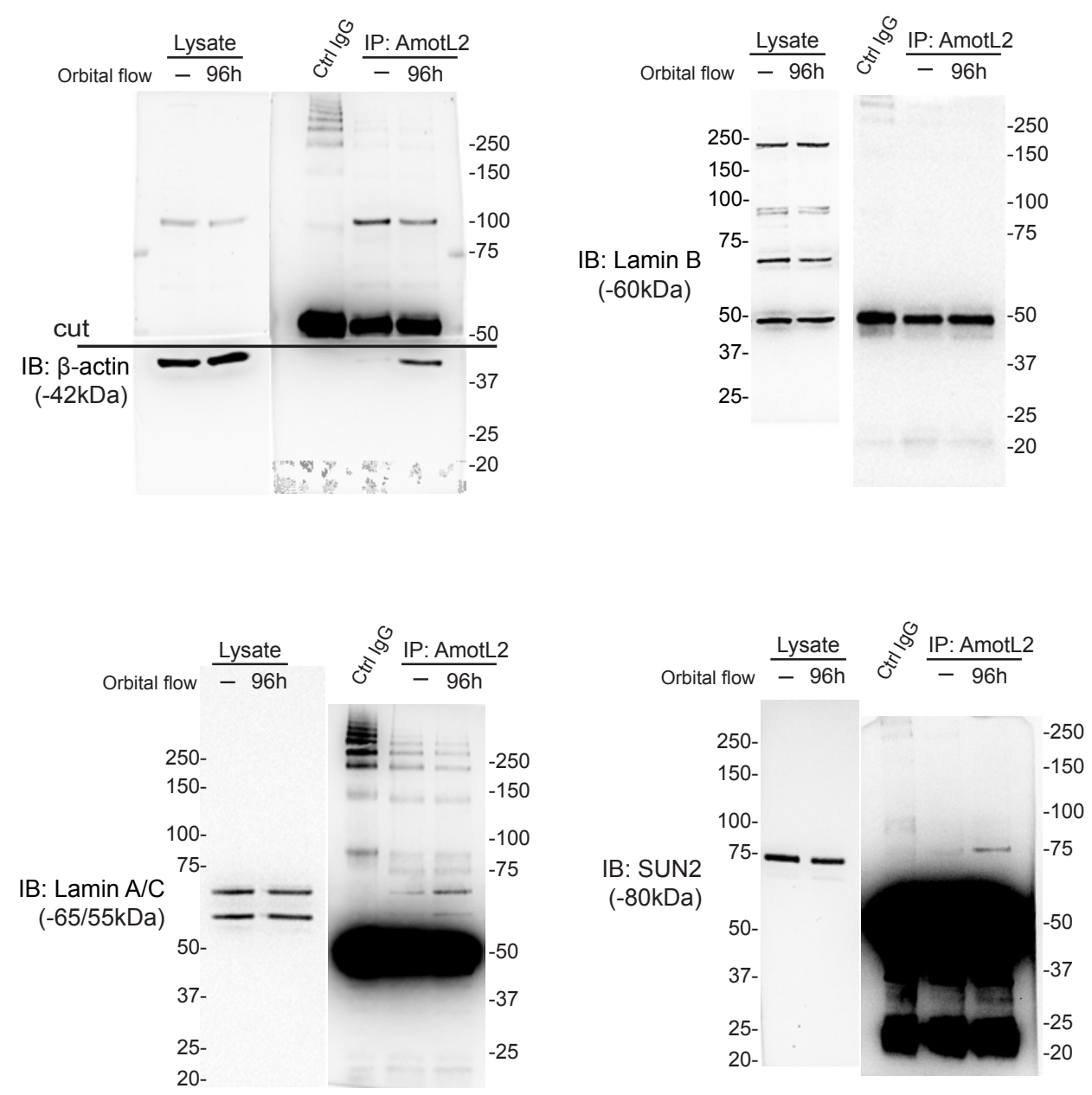


\begin{tabular}{|c|c|c|c|c|c|c|c|}
\hline Accession & Description & Area_Ctrl_1 & Area_Ctrl_2 & Area_Ctrl_3 & Area_AmotL2_1 & Area_Amott2_2 & Area_AmotL2_3 \\
\hline P99027 & $60 \mathrm{~S}$ acidic ribosomal protein P2 OS=Mus musculus GN=Rplp2 PE=1 SV=3 - [RLA2_MOUSE] & $1,197 \mathrm{E}$ & $0,000 \mathrm{E} 0$ & $0,000 \mathrm{E} 0$ & $1,025 E 8$ & \begin{tabular}{l|l}
$1,555 \mathrm{E} 8$ \\
\end{tabular} & $5,822 E 8$ \\
\hline P62204 & Calmodulin OS=Mus musculus GN=Calm1 PE=1 SV=2 - [CALM_MOUSE] & $7,282 E 7$ & $0,000 E 0$ & $8,936 \mathrm{E} 7$ & $1,808 E 9$ & $4,501 \mathrm{Eg}$ & 9,019E9 \\
\hline P58771-2 & Isoform 2 of Tropomyosin alpha-1 chain OS=Mus musculus GN=Tpm1 - [TPM1_MOUSE] & $0,000 E 0$ & $0,000 E 0$ & $1,738 \mathrm{E} 7$ & $1,752 \mathrm{Eg}$ & $2,676 \mathrm{Eg}$ & 4,298E9 \\
\hline P60710 & Actin, cytoplasmic 1 OS=Mus musculus GN=Actb PE=1 SV=1 - [ACTB_MOUSE] & $9,315 \mathrm{E} 8$ & $8,508 \mathrm{E} 5$ & $2,323 E 8$ & 1,154E10 & $2,141 \mathrm{E} 10$ & $1,591 E 10$ \\
\hline Q60605 & Myosin light polypeptide $6 \mathrm{OS}=$ Mus musculus $\mathrm{GN}=$ Myl6 PE=1 SV=3 - [MYL6_MOUSE] & $3,418 E 8$ & $0,000 \mathrm{E} 0$ & $1,038 \mathrm{E} 8$ & 7,357E9 & $1,214 E 10$ & $1,374 E 10$ \\
\hline Q60605-2 & Isoform Smooth muscle of Myosin light polypeptide 6 OS=Mus musculus GN=Myl6 - [MYL6_MOUSE] & $3,354 \mathrm{E} 8$ & $0,000 \mathrm{E} 0$ & $1,038 E 8$ & $7,357 \mathrm{E} 9$ & $1,214 \mathrm{E} 10$ & $1,374 E 10$ \\
\hline Q9נJU8 & SH3 domain-binding glutamic acid-rich-like protein OS=Mus musculus GN=Sh3bgrl PE=1 SV=1 - [SH3L1_MOUSE] & $0,000 \mathrm{E} 0$ & $0,000 E 0$ & $0,000 E_{0}$ & $2,505 E 6$ & $1,384 E 7$ & 7,620E7 \\
\hline P20152 & Vimentin $\mathrm{OS}=$ Mus musculus $\mathrm{GN}=\mathrm{Vim} \mathrm{PE}=1 \mathrm{SV}=3$ - [VIME_MOUSE] & 6,052E7 & $0,000 E 0$ & $4,966 \mathrm{E} 7$ & $1,116 \mathrm{E} 8$ & $2,178 \mathrm{E} 8$ & $1,545 \mathrm{Eg}$ \\
\hline P63168 & Dynein light chain 1, cytoplasmic OS=Mus musculus GN=Dynll1 PE=1 SV=1 - [DYL1_MOUSE] & $0,000 \mathrm{E} 0$ & $0,000 \mathrm{E} 0$ & $9,000 \mathrm{E} 5$ & $6,575 E 7$ & 3,637E6 & $2,389 E 6$ \\
\hline P62908 & $40 S$ ribosomal protein S3 OS=Mus musculus GN=Rps3 PE=1 SV=1 - [RS3_MOUSE] & $2,511 \mathrm{E} 7$ & $0,000 \mathrm{E} 0$ & $1,247 \mathrm{E}$ & $5,806 E 8$ & 1,105E9 & 2,353E9 \\
\hline P18760 & Cofilin-1 OS=Mus musculus GN=Cf11 PE=1 SV=3 - [COF1_MOUSE] & $0,000 \mathrm{E} 0$ & $0,000 \mathrm{E} 0$ & $1,219 \mathrm{E} 7$ & $2,350 \mathrm{E} 8$ & $6,076 E_{8}$ & $5,269 \mathrm{E} 8$ \\
\hline Q6IRU2 & Tropomyosin alpha-4 chain OS=Mus musculus GN=Tpm4 PE=1 $\mathrm{SV}=3$ - [TPM4_MOUSE] & $0,000 \mathrm{E} 0$ & $0,000 E 0$ & $1,218 \mathrm{E} 7$ & $7,452 E 8$ & $6,204 E 8$ & $9,875 E 8$ \\
\hline Q3THE2 & Myosin regulatory light chain $12 \mathrm{~B}$ OS=Mus musculus GN=My12b PE=1 SV=2 - [ML12B_MOUSE] & $1,647 \mathrm{E}$ & $0,000 E 0$ & $1,331 \mathrm{E} 8$ & $5,052 \mathrm{Eg}$ & $8,498 \mathrm{Eg}$ & $1,342 E 10$ \\
\hline Q8VDD5 & Myosin-9 OS=Mus musculus GN=Myh9 PE=1 SV=4 - [MYH9_MOUSE] & $7,800 E 8$ & $0,000 E_{0}$ & $3,751 \mathrm{E} 8$ & $1,395 E 10$ & $2,268 E 10$ & $1,618 E 10$ \\
\hline P63101 & 14-3-3 protein zeta/delta OS=Mus musculus GN=Ywhaz PE=1 SV=1 - [1433Z_MOUSE] & $0,000 E 0$ & $0,000 E 0$ & $5,426 \mathrm{E} 6$ & $1,197 \mathrm{E}$ & $2,006 \mathrm{E} 8$ & $3,279 E 8$ \\
\hline Q9WTI7-3 & Isoform 3 of Unconventional myosin-Ic OS=Mus musculus GN=Myo1c - [MYO1C_MOUSE] & 5,544E7 & $0,000 \mathrm{E} 0$ & 4,243E7 & $1,796 \mathrm{Eg}$ & 4,341E9 & $1,165 E 10$ \\
\hline Q9WTI7 & Unconventional myosin-Ic OS=Mus musculus GN=Myo1c PE=1 SV=2 - [MYO1C_MOUSE] & 5,544E7 & $0,000 \mathrm{E} 0$ & 4,243E7 & $1,796 \mathrm{Eg}$ & $4,341 \mathrm{Eg}$ & $1,165 E 10$ \\
\hline Q9WVK4 & EH domain-containing protein $1 \mathrm{OS}=$ Mus musculus $\mathrm{GN}=\mathrm{Ehd} 1 \mathrm{PE}=1 \mathrm{SV}=1$ - [EHD1_MOUSE] & $0,000 \mathrm{E} 0$ & $0,000 E 0$ & $0,000 E 0$ & $1,740 \mathrm{E} 8$ & $2,243 E 8$ & $3,573 \mathrm{E}^{2}$ \\
\hline P21107-2 & Isoform 2 of Tropomyosin alpha-3 chain OS=Mus musculus GN=Tpm3 - [TPM3_MOUSE] & 2,921E7 & $0,000 \mathrm{E} 0$ & $1,212 E 7$ & $2,040 \mathrm{E} 9$ & 3,139E9 & 4,747E9 \\
\hline P62259 & 14-3-3 protein epsilon OS=Mus musculus $\mathrm{GN}=$ Ywhae PE=1 SV=1 - [1433E_MOUSE] & $0,000 \mathrm{E}_{0}$ & $0,000 \mathrm{E}_{0}$ & 4,263E6 & $1,606 \mathrm{E} 8$ & 3,051E8 & $5,462 E 8$ \\
\hline P47757-2 & Isoform 2 of F-actin-capping protein subunit beta OS=Mus musculus GN=Capzb - [CAPZB_MOUSE] & $0,000 \mathrm{E}_{0}$ & $0,000 \mathrm{E} 0$ & $0,000 \mathrm{E} 0$ & $2,392 E 8$ & 5,788E8 & $8,469 \mathrm{E} 8$ \\
\hline P62702 & 40 S ribosomal protein S4, X isoform OS=Mus musculus GN=Rps4X PE=1 SV=2 - [RS4X_MOUSE] & $0,000 E 0$ & $0,000 E 0$ & $0,000 \mathrm{E} 0$ & 4,817Е8 & 8,993E8 & $1,160 \mathrm{Eg}$ \\
\hline P59999 & Actin-related protein 2/3 complex subunit 4 OS $=$ Mus musculus GN=Arpc4 PE $=1 \mathrm{SV}=3-$ [ARPC4_MOUSE] & $0,000 E_{0}$ & $0,000 \mathrm{E} 0$ & $0,0000_{0}$ & $1,485 \mathrm{E} 8$ & $3,024 E 8$ & $4,275 E 8$ \\
\hline P47754 & F-actin-capping protein subunit alpha-2 $\mathrm{OS}=$ Mus musculus $\mathrm{GN}=\mathrm{Capza2} \mathrm{PE}=1 \mathrm{SV}=3$ - [CAZA2_MOUSE] & $0,000 \mathrm{E} 0$ & $0,000 \mathrm{E} 0$ & 2,21455 & $1,560 \mathrm{E} 8$ & $3,100 \mathrm{E} 8$ & 2,515E8 \\
\hline Q9D0M5 & Dynein light chain 2, cytoplasmic OS=Mus musculus GN=Dynl12 PE=1 SV=1 - [DYL2_MOUSE] & $0,000 E_{0}$ & $0,000 \mathrm{E}_{0}$ & $1,134 E 6$ & $3,076 \mathrm{E} 7$ & 4,492E6 & $2,203 E 6$ \\
\hline Q61879 & Myosin-10 OS=Mus musculus GN=Myh10 PE=1 SV=2 - [MYH10_MOUSE] & $1,848 E 8$ & $0,000 E 0$ & 7,692E7 & $5,644 \mathrm{Eg}$ & 9,609E9 & 6,899E9 \\
\hline$P 68040$ & Receptor of activated protein C kinase 1 OS=Mus musculus $\mathrm{GN}=$ Rack1 PE=1 SV=3 - [RACK1_MOUSE] & $0,000 \mathrm{E} 0$ & $0,000 E 0$ & $7,614 E 6$ & $2,549 E 8$ & $4,560 \mathrm{E} 8$ & $1,075 \mathrm{E} 9$ \\
\hline P47757 & F-actin-capping protein subunit beta OS=Mus musculus GN=Capzb PE $=1 \mathrm{SV}=3$ - [CAPZB_MOUSE] & $0,000 \mathrm{E} 0$ & $0,000 \mathrm{E} 0$ & $0,000 \mathrm{E} 0$ & $2,392 E 8$ & 5,788E8 & $8,469 \mathrm{E} 8$ \\
\hline P57780 & Alpha-actinin-4 OS=Mus musculus GN=Actn4 PE=1 SV=1 - [ACTN4_MOUSE] & $0,000 E 0$ & $0,000 E 0$ & 3,995E7 & $6,351 E 7$ & $2,203 E 8$ & $3,475 E 8$ \\
\hline Q9CQV8-2 & Isoform Short of 14-3-3 protein beta/alpha OS=Mus musculus GN=Ywhab - [1433B_MOUSE] & $0,000 E 0$ & $0,000 E 0$ & $3,951 \mathrm{E} 6$ & $8,622 E 7$ & 9,673E7 & $1,169 \mathrm{E} 8$ \\
\hline P10854 & Histone H2B type 1-M OS=Mus musculus GN=Hist1h2bm PE=1 SV=2 - [H2B1M_MOUSE] & $0,000 E 0$ & $0,000 E 0$ & $3,713 \mathrm{E} 6$ & $5,988 \mathrm{E} 7$ & 6,547E7 & $2,447 \pm 8$ \\
\hline P14131 & 405 ribosomal protein $\mathrm{S} 16 \mathrm{OS}=$ Mus musculus GN=Rps16 PE=1 SV=4 - [RS16_MOUSE] & $0,000 E 0$ & $0,000 E_{0}$ & $3,807 E 6$ & $3,581 \mathrm{E} 8$ & $6,287 \mathrm{E}$ & $1,211 \mathrm{Eg}$ \\
\hline P62889 & 60S ribosomal protein L30 OS=Mus musculus GN=Rpl30 PE=1 SV=2 - [RL30_MOUSE] & $0,000 \mathrm{E} 0$ & $0,000 \mathrm{E} 0$ & $3,622 \mathrm{E} 6$ & $1,217 \mathrm{E}$ & 3,463E8 & $4,716 \mathrm{E} 8$ \\
\hline P62962 & Profflin-1 OS=Mus musculus GN=Pfn1 PE=1 SV=2 - [PROF1_MOUSE] & $0,000 \mathrm{E} 0$ & $0,000 E_{0}$ & $0,000 E 0$ & $6,477 E 7$ & $5,550 \mathrm{E} 7$ & $1,591 \mathrm{E}$ \\
\hline P26041 & Moesin OS=Mus musculus GN=Msn PE=1 SV=3 - [MOES_MOUSE] & $0,000 \mathrm{E} 0$ & $0,000 \mathrm{E} 0$ & $8,796 \mathrm{E} 5$ & $1,725 \mathrm{~EB}$ & 6,499E8 & $8,865 E 8$ \\
\hline Q9WTX5 & S-phase kinase-associated protein 1 OS=Mus musculus GN=Skp1 PE=1 SV=3 - [SKP1_MOUSE] & $0,000 E 0$ & $0,000 E 0$ & 3,37766 & $1,064 \mathrm{E}$ & $4,061 \mathrm{E7}$ & 7,292E7 \\
\hline Q9QXS1-3 & Isoform PLEC-1A of Plectin OS=Mus musculus GN=Plec - [PLEC_MOUSE] & $0,000 E 0$ & $0,000 E 0$ & $3,986 \mathrm{E} 6$ & $3,329 \mathrm{E}$ & $7,844 \mathrm{E} 8$ & $1,021 \mathrm{Eg}$ \\
\hline P39447 & Tight junction protein ZO-1 OS=Mus musculus GN=Tjp1 PE=1 SV=2 - [ZO1_MOUSE] & $0,000 E 0$ & $0,000 E 0$ & $1,395 E 5$ & $5,906 \mathrm{E} 8$ & $1,169 \mathrm{Eg}$ & $7,026 \mathrm{E}$ \\
\hline Q9CPW4 & Actin-related protein 2/3 complex subunit 5 OS=Mus musculus GN=Arpc5 PE=1 SV=3 - [ARPC5_MOUSE] & $0,000 \mathrm{E} 0$ & $0,000 \mathrm{E} 0$ & $0,000 \mathrm{E} 0$ & $3,182 \mathrm{E} 7$ & $5,082 \mathrm{E7}$ & $1,035 E_{8}$ \\
\hline Q9WVA4 & Transgelin-2 OS=Mus musculus GN=Tagln2 PE=1 SV=4 - [TAGL2_MOUSE] & $0,000 \mathrm{E}_{0}$ & $0,000 \mathrm{E} 0$ & $0,000 E_{0}$ & $1,844 \mathrm{E} 7$ & 5,034E7 & 9,225E7 \\
\hline Q9EQP2 & EH domain-containing protein $4 \mathrm{OS}=$ Mus musculus $\mathrm{GN}=$ Ehd4 $\mathrm{PE}=1 \mathrm{SV}=1$ - [EHD4_MOUSE] & $0,000 E 0$ & $0,000 \mathrm{E} 0$ & $1,218 E 6$ & $2,279 E 8$ & $3,490 \mathrm{E} 8$ & 4,719E8 \\
\hline P62830 & 60S ribosomal protein L23 OS=Mus musculus GN=Rpl23 PE=1 SV=1 - [RL23_MOUSE] & $0,000 E 0$ & $0,000 E 0$ & $1,581 \mathrm{E}$ & $1,267 \pm 8$ & $2,247 \mathrm{E}$ & $2,290 \mathrm{E} 8$ \\
\hline P45591 & Coffilin-2 OS=Mus musculus GN=Cf2 PE=1 SV=1 - [COF2_MOUSE] & $0,000 \mathrm{E} 0$ & $0,000 \mathrm{E} 0$ & $1,310 \mathrm{E} 7$ & $2,582 \mathrm{E} 8$ & 3,874E8 & 2,411E8 \\
\hline Q7TPR4 & Alpha-actinin-1 OS=Mus musculus GN=Actn1 PE=1 SV=1 - [ACTN1_MOUSE] & $0,000 \mathrm{E}_{0}$ & $0,000 \mathrm{E}_{0}$ & 3,995E7 & $6,662 E 7$ & $2,352 \mathrm{E} 8$ & 3,356E8 \\
\hline Q9QXS1-2 & Isoform PLEC-1 of Plectin OS=Mus musculus GN=Plec - [PLEC_MOUSE] & $0,000 \mathrm{E} 0$ & $0,000 E 0$ & $3,986 \mathrm{E} 6$ & $3,329 \mathrm{E} 8$ & $7,844 E 8$ & $1,021 \mathrm{Eg}$ \\
\hline P30999 & Catenin delta-1 $\mathrm{OS}=$ Mus musculus $\mathrm{GN}=\mathrm{Ctnnd1} \mathrm{PE}=1 \mathrm{SV}=2$ - [CTND1_MOUSE] & $0,000 \mathrm{E} 0$ & $0,000 \mathrm{E} 0$ & 3,219E6 & $1,240 E 8$ & $3,330 \mathrm{E} 8$ & $4,168 E 8$ \\
\hline Q8BH64 & EH domain-containing protein $2 \mathrm{OS}=$ Mus musculus $\mathrm{GN}=\mathrm{Ehd} 2 \mathrm{PE}=1 \mathrm{SV}=1$ - [EHD2_MOUSE] & $0,000 E 0$ & $0,000 E 0$ & $0,000 E 0$ & 9,033 E & $1,842 \mathrm{E} 8$ & $2,833 E 8$ \\
\hline P26231 & Catenin alpha-1 $\mathrm{OS}=$ Mus musculus GN=Ctnna1 $\mathrm{PE}=1 \mathrm{SV}=1$ - [CTNA1_MOUSE] & $0,000 E 0$ & $0,000 E 0$ & $0,000 E 0$ & $2,894 E 8$ & $4,583 \mathrm{E} 8$ & 5,492E8 \\
\hline P51410 & $60 S$ ribosomal protein L9 OS=Mus musculus GN=Rpl9 PE=2 SV=2 - [RL9_MOUSE] & $0,000 \mathrm{E}_{0}$ & $0,000 E 0$ & $0,0000_{0}$ & $1,632 E 8$ & $2,751 \mathrm{E} 8$ & $2,248 E 8$ \\
\hline P62264 & 40 S ribosomal protein $\mathrm{S} 14$ OS=Mus musculus GN=Rps14 PE=1 SV=3 - [RS14_MOUSE] & $0,000 E 0$ & $0,000 E 0$ & $8,814 E 6$ & $3,426 \mathrm{E} 8$ & $1,848 \mathrm{E} 8$ & $1,001 \mathrm{Eg}$ \\
\hline Q68FD5 & Clathrin heavy chain 1 OS=Mus musculus GN=Cltc PE=1 SV=3 - [CLH1_MOUSE] & $0,000 E 0$ & $0,000 E 0$ & 5,093E6 & 9,943E8 & 1,302E9 & $1,498 \mathrm{Eg}$ \\
\hline P35979 & 605 ribosomal protein L12 OS=Mus musculus GN=Rpl12 PE=1 SV=2 - [RL12_MOUSE] & $1,348 \mathrm{E} 7$ & $0,000 \mathrm{E}_{0}$ & $3,911 \mathrm{E} 6$ & $2,635 \mathrm{~EB}$ & 5,822E8 & $9,416 \mathrm{E} 8$ \\
\hline P60843 & Eukaryotic initiation factor 4A-I OS=Mus musculus GN=Eif4a1 PE=1 SV=1 - [IF4A1_MOUSE] & $0,000 \mathrm{E} 0$ & $0,000 \mathrm{E} 0$ & $3,565 E 5$ & 7,572E7 & $1,578 E 8$ & $2,085 E 8$ \\
\hline Q91WK0 & Leucine-rich repeat flightless-interacting protein 2 OS=Mus musculus GN=Lrfip2 PE=1 SV=1 - [LRRF2_MOUSE] & $0,000 \mathrm{E}_{0}$ & $0,000 \mathrm{E} 0$ & $1,370 \mathrm{E}$ & $1,962 \mathrm{E} 8$ & 3,153E8 & $2,418 E 8$ \\
\hline P17182 & Alpha-enolase OS=Mus musculus GN=Eno1 PE=1 SV=3 - [ENOA_MOUSE] & $0,000 \mathrm{E} 0$ & $0,000 \mathrm{E} 0$ & $3,821 \mathrm{E}$ & $4,461 \mathrm{E7}$ & $1,095 \mathrm{E} 8$ & $1,119 \mathrm{~EB}$ \\
\hline P84228 & Histone $\mathrm{H} 3.2 \mathrm{OS}=$ Mus musculus $\mathrm{GN}=$ Hist1h3b PE=1 SV=2 - [H32_MOUSE] & $0,000 \mathrm{E} 0$ & $0,000 E_{0}$ & 6,923E6 & $5,171 \mathrm{E}$ & 9,432E7 & $5,068 \mathrm{E} 8$ \\
\hline P63242 & Eukaryotic translation initiation factor 5A-1 OS=Mus musculus GN=Eif5a PE=1 SV=2 - [IF5A1_MOUSE] & $0,000 \mathrm{E} 0$ & $0,000 E 0$ & $0,000 E_{0}$ & $1,968 E 7$ & $8,325 \mathrm{E} 7$ & 9,981E7 \\
\hline P21107 & Tropomyosin alpha-3 chain OS=Mus musculus GN=Tpm3 PE=1 $\mathrm{SV}=3$ - [TPM3_MOUSE] & $1,721 \mathrm{E} 7$ & $0,000 E_{0}$ & $8,255 E 6$ & $1,895 \mathrm{Eg}$ & $2,603 E 9$ & $4,298 \mathrm{EE}$ \\
\hline Q999Y9 & Actin-related protein 3 OS=Mus musculus $\mathrm{GN}=\mathrm{Actr} 3 \mathrm{PE}=1 \mathrm{SV}=3-$ [ARP3_MOUSE] & $0,000 \mathrm{E} 0$ & $0,000 \mathrm{E}_{0}$ & $2,151 \mathrm{E} 6$ & $9,174 E 7$ & $2,808 E 8$ & $3,414 E 8$ \\
\hline Q8BQ30 & Phostensin OS=Mus musculus GN=Ppp1r18 PE=1 SV=1 - [PPR18_MOUSE] & $0,000 E_{0}$ & $0,000 E 0$ & $0,000 E 0$ & $8,519 E 7$ & $2,448 E 8$ & $5,048 E 8$ \\
\hline E9Q634 & Unconventional myosin-Ie OS=Mus musculus GN=Myo1e PE=1 SV=1 - [MYO1E_MOUSE] & $0,000 E 0$ & $0,000 E 0$ & $2,601 \mathrm{E} 6$ & $2,534 E 8$ & $4,588 \mathrm{E} 8$ & $8,539 \mathrm{~EB}$ \\
\hline P97351 & $40 S$ ribosomal protein S3a OS=Mus musculus GN=Rps3a PE=1 SV=3 - [RS3A_MOUSE] & $0,000 \mathrm{E} 0$ & $0,000 E_{0}$ & $3,947 \pm 6$ & $2,516 \mathrm{E} 8$ & 4,596E8 & 9,725E8 \\
\hline P62245 & $40 S$ ribosomal protein S15a OS=Mus musculus GN=Rps15a PE=1 SV=2 - [RS15A_MOUSE] & $0,000 E_{0}$ & $0,000 \mathrm{E} 0$ & $0,000 \mathrm{E} 0$ & $2,970 \mathrm{E} 8$ & 5,802E8 & $7,716 \mathrm{E}$ \\
\hline P23506 & Protein-L-isoaspartate(D-aspartate) O-methyltransferase OS=Mus musculus GN=Pcmt1 PE $=1 \mathrm{SV}=3$ - [PIMT_MOUSE] & $0,000 E_{0}$ & $0,000 E_{0}$ & $3,447 E 5$ & 4,734E7 & 5,273E7 & 4,213E7 \\
\hline P16546-2 & Isoform 2 of Spectrin alpha chain, non-erythrocytic 1 OS=Mus musculus GN=Sptan1 - [SPTN1_MOUSE] & $0,000 E 0$ & $0,000 E 0$ & $0,000 E 0$ & 7,210E7 & $1,351 E 8$ & $1,963 \mathrm{E}$ \\
\hline Q9CVB6 & Actin-related protein $2 / 3$ complex subunit 2 OS=Mus musculus GN=Arpc2 PE=1 SV=3 - [ARPC2_MOUSE] & $0,000 E 0$ & $0,000 E 0$ & $0,000 E 0$ & $9,702 E 7$ & $3,457 \mathrm{E} 8$ & 5,493E8 \\
\hline Q9JKB3-2 & Isoform 2 of $\mathrm{Y}$-box-binding protein 3 OS=Mus musculus $\mathrm{GN}=\mathrm{Yb} \times 3$ - [YBOX3_MOUSE] & $0,000 E 0$ & $0,000 E 0$ & $0,000 E 0$ & $1,701 E 8$ & $3,202 E 8$ & $7,389 \mathrm{~EB}$ \\
\hline P16546 & Spectrin alpha chain, non-erythrocytic 1 OS=Mus musculus GN=Sptan1 PE=1 SV=4 - [SPTN1_MOUSE] & $0,000 \mathrm{E} 0$ & $0,000 \mathrm{E} 0$ & $0,0000_{0}$ & $7,210 E 7$ & $1,351 \mathrm{E} 8$ & $1,963 E 8$ \\
\hline P63017 & Heat shock cognate $71 \mathrm{kDa}$ protein $\mathrm{OS}=$ Mus musculus $\mathrm{GN}=\mathrm{Hspa} 8 \mathrm{PE}=1 \mathrm{SV}=1$ - [HSP7C_MOUSE] & 3,744E7 & $0,000 \mathrm{E} 0$ & $1,198 \mathrm{E} 7$ & $6,088 \mathrm{E} 8$ & $1,368 \mathrm{Eg}$ & $2,139 \mathrm{Eg}$ \\
\hline P61750 & ADP-ribosylation factor $4 \mathrm{OS}=$ Mus musculus GN=Arf4 PE $=1 \mathrm{SV}=2$ - [ARF4_MOUSE] & $0,000 \mathrm{E} 0$ & $0,000 E_{0}$ & $7,116 \mathrm{E}$ & $2,373 E 7$ & 5,966E7 & 9,349E7 \\
\hline Q9Z0U1 & Tight junction protein ZO-2 OS=Mus musculus GN=Tjp2 PE=1 SV=2 - [ZO2_MOUSE] & $0,000 E_{0}$ & $0,000 E_{0}$ & $2,474 E 6$ & $5,557 \mathrm{E} 8$ & 1,315E9 & $1,020 \mathrm{Eg}$ \\
\hline 054962 & Barrier-to-autointegration factor OS=Mus musculus GN=Banf1 PE $=1 \mathrm{SV}=1$ - [BAF_MOUSE] & $0,000 \mathrm{E} 0$ & $0,000 \mathrm{E} 0$ & $0,000 \mathrm{E} 0$ & $4,798 \mathrm{E} 6$ & $7,358 \mathrm{E7}$ & $1,607 E 8$ \\
\hline Q9נHJ0 & Tropomodulin-3 OS=Mus musculus GN=Tmod3 PE=1 SV=1 - [TMOD3_MOUSE] & $0,000 \mathrm{E} 0$ & $0,000 \mathrm{E} 0$ & $1,356 \mathrm{E}$ & $3,738 \mathrm{E} 8$ & 7,196E8 & $9,605 E 8$ \\
\hline P14869 & $60 \mathrm{~S}$ acidic ribosomal protein P0 OS=Mus musculus GN=Rplp0 PE=1 SV=3 - [RLA0_MOUSE] & 4,904E7 & $0,000 E 0$ & $0,000 \mathrm{E} 0$ & $1,401 E 8$ & $3,082 \mathrm{E} 8$ & $8,169 \mathrm{E} 8$ \\
\hline Q9CZX8 & 40 S ribosomal protein $\mathrm{S19}$ OS=Mus musculus GN=Rps19 PE=1 SV=3 - [RS19_MOUSE] & $0,000 \mathrm{E} 0$ & $0,000 E_{0}$ & $1,201 E 7$ & $2,159 \mathrm{E} 8$ & $3,565 \mathrm{E} 8$ & 7,484E8 \\
\hline Q64337 & Sequestosome-1 OS=Mus musculus GN=Sqstm1 PE=1 SV=1 - [SQSTM_MOUSE] & $0,000 E 0$ & $0,000 E 0$ & $0,000 \mathrm{E}_{0}$ & $1,741 \mathrm{E} 7$ & 1,819E8 & $1,250 E 8$ \\
\hline P62806 & Histone H4 OS=Mus musculus $\mathrm{GN}=$ Hist1h4a PE=1 SV=2 - [H4_MOUSE] & $3,548 \mathrm{E} 7$ & $0,000 E_{0}$ & $2,100 E 6$ & $4,491 E 7$ & $1,256 \mathrm{E} 8$ & $1,525 \mathrm{Eg}$ \\
\hline P14148 & $60 S$ ribosomal protein L7 OS=Mus musculus GN=Rp17 PE=1 SV=2 - [RL7_MOUSE] & $0,000 \mathrm{E} 0$ & $0,000 \mathrm{E} 0$ & $8,463 E 5$ & $1,918 \mathrm{E} 8$ & $3,841 \mathrm{E} 8$ & $1,158 \mathrm{Eg}$ \\
\hline Q62261 & Spectrin beta chain, non-erythrocytic $10 \mathrm{OS}=$ Mus musculus GN=Sptbn1 PE=1 SV=2 - [SPTB2_MOUSE] & $0,000 E_{0}$ & $0,000 \mathrm{E} 0$ & $2,966 \mathrm{E} 6$ & $5,610 \mathrm{E} 7$ & $1,701 \mathrm{E}$ & $2,578 E 8$ \\
\hline Q61937 & Nucleophosmin OS=Mus musculus GN=Npm1 PE=1 SV=1 - [NPM_MOUSE] & $0,000 \mathrm{E} 0$ & $0,000 \mathrm{E} 0$ & $1,406 \mathrm{E}$ & $4,148 E 8$ & $3,164 \mathrm{E} 8$ & $3,566 \mathrm{E} 8$ \\
\hline P68254 & 14-3-3 protein theta OS=Mus musculus GN=Ywhaq PE=1 SV=1 $-\left[1433 T \_\right.$MOUSE] & $0,000 \mathrm{E} 0$ & $0,000 \mathrm{E} 0$ & $5,736 \mathrm{E}$ & 6,738E7 & $8,293 E 7$ & $1,954 \mathrm{E} 8$ \\
\hline Q9WU78 & Programmed cell death 6-interacting protein OS=Mus musculus GN=Pdcd6ip PE=1 SV=3 - [PDC6I_MOUSE] & $0,000 E_{0}$ & $0,000 \mathrm{E} 0$ & $2,031 E 5$ & $1,063 \mathrm{E} 8$ & $2,196 \mathrm{E} 8$ & 3,132E8 \\
\hline Q62261-2 & Isoform 2 of Spectrin beta chain, non-erythrocytic 1 OS=Mus musculus GN=Sptbn1 - [SPTB2_MOUSE] & $0,000 \mathrm{E} 0$ & $0,000 \mathrm{E} 0$ & $2,966 \mathrm{E} 6$ & $5,538 \mathrm{E} 7$ & $1,701 \mathrm{E} 8$ & $2,578 E 8$ \\
\hline P61255 & 605 ribosomal protein L26 OS=Mus musculus GN=Rpl26 PE=1 SV=1 - [RL26_MOUSE] & $0,000 E_{0}$ & $0,000 \mathrm{E} 0$ & $0,000 \mathrm{E} 0$ & $2,152 E 8$ & $2,489 \mathrm{E}$ & $7,882 E 8$ \\
\hline P61982 & 14-3-3 protein gamma OS=Mus musculus GN=Ywhag PE=1 SV=2 - [1433G_MOUSE] & $0,000 \mathrm{E} 0$ & $0,000 \mathrm{E} 0$ & $0,000 E 0$ & 1,573E8 & $1,548 E 8$ & $1,471 E 8$ \\
\hline P97434-2 & Isoform 2 of Myosin phosphatase Rho-interacting protein OS=Mus musculus GN=Mprip - [MPRIP_MOUSE] & $0,000 E_{0}$ & $0,000 E 0$ & $0,000 E_{0}$ & $1,158 \mathrm{E} 8$ & $1,846 \mathrm{E} 8$ & $2,425 \mathrm{E}$ \\
\hline P62082 & $40 S$ ribosomal protein S7 OS=Mus musculus GN=Rps7 PE=2 SV=1 - [RS7_MOUSE] & $0,000 E_{0}$ & $0,000 \mathrm{E}_{0}$ & $1,346 \mathrm{E} 7$ & $1,913 \mathrm{~EB}$ & $1,777 \mathrm{E}$ & 3,225E8 \\
\hline P62270 & $40 S$ ribosomal protein $\mathrm{S} 18 \mathrm{OS}=$ Mus musculus GN=Rps18 PE=1 SV=3 - [RS18_MOUSE] & $0,000 E 0$ & $0,000 E_{0}$ & $5,188 \mathrm{E} 6$ & $2,852 E 8$ & $2,371 \mathrm{E} 8$ & 9,919E8 \\
\hline Q9EP71 & Ankycorbin OS=Mus musculus GN=Rai14 PE=1 SV=1 - [RAI14_MOUSE] & $0,000 E_{0}$ & $0,000 \mathrm{E} 0$ & $0,000 E_{0}$ & $1,024 E 8$ & $1,644 \mathrm{E} 8$ & $2,120 \mathrm{E} 8$ \\
\hline Q6ZWV3 & 60 ribosomal protein L10 OS=Mus musculus GN=Rp110 PE=1 SV=3 - [RL10_MOUSE] & $0,000 E_{0}$ & $0,000 \mathrm{E} 0$ & 3,303E6 & $1,397 \mathrm{E}$ & $3,848 E 8$ & 7,309E8 \\
\hline
\end{tabular}




\begin{tabular}{|c|c|c|c|c|c|}
\hline Q62167 & ATP-dependent RNA helicase DDX3X OS=Mus musculus GN=Ddx3X PE=1 SV=3 - [DDX3X_MOUSE] & $1,704 E 6$ & $0,000 \mathrm{E} 0$ & 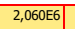 & $1,659 \mathrm{E} 8$ \\
\hline Q9CPR4 & $60 S$ ribosomal protein L17 OS=Mus musculus GN=Rpl17 PE=1 SV=3 - [RL17_MOUSE] & $0,000 E 0$ & $0,000 E 0$ & $0,000 \mathrm{EO}$ & $2,699 \mathrm{E} 8$ \\
\hline Q8C143 & Myosin light chain 6B OS=Mus musculus GN=Myl6b PE=1 SV=1 - [MYL6B_MOUSE] & $7,368 \mathrm{E} 8$ & $0,000 \mathrm{E} 0$ & $1,897 E 8$ & 4,242E9 \\
\hline Q2KN98 & Cytospin-A OS=Mus musculus GN=Specc11 PE=1 SV=1 - [CYTSA_MOUSE] & $0,000 E 0$ & $0,000 E 0$ & $0,000 \mathrm{E} 0$ & $1,569 \mathrm{E} 8$ \\
\hline P16858 & Glyceraldehyde-3-phosphate dehydrogenase OS=Mus musculus GN=Gapdh PE=1 SV=2 - [G3P_MOUSE] & $8,286 \mathrm{E}$ & $0,000 \mathrm{E} 0$ & 3,409E6 & 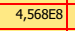 \\
\hline Q9QYB1 & Chloride intracellular channel protein 4 OS=Mus musculus GN=Clic4 PE=1 SV=3 - [CLIC4_MOUSE] & $0,000 E 0$ & $0,000 \mathrm{E}_{0}$ & 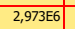 & $1,351 \mathrm{E} 7$ \\
\hline Q8CGP6 & Histone H2A type 1 -H OS=Mus musculus GN=Hist1h2ah PE=1 SV=3 - [H2A1H_MOUSE] & 1,458E7 & $9,648 E 6$ & $2,742 E 7$ & $5,055 \mathrm{E}]$ \\
\hline P47753 & F-actin-capping protein subunit alpha-1 OS=Mus musculus GN=Capza1 PE=1 SV=4 - [CAZA1_MOUSE] & $0,0000_{0}$ & $0,000 \mathrm{EO}$ & $0,000 \mathrm{E}_{0}$ & $1,417 E 8$ \\
\hline P35700 & Peroxiredoxin-1 OS=Mus musculus GN=Prdx1 PE=1 SV=1 - [PRDX1_MOUSE] & $0,000 E 0$ & $0,000 \mathrm{E} 0$ & $0,000 \mathrm{E} 0$ & $1,399 \mathrm{E}$ \\
\hline P52480 & Pyruvate kinase PKM OS=Mus musculus GN=Pkm PE=1 SV=4 - [KPYM_MOUSE] & $0,000 E 0$ & $0,000 E_{0}$ & $1,563 \mathrm{E} 7$ & $3,242 \mathrm{E}$ \\
\hline Q9JHL1-2 & Isoform 2 of $\mathrm{Na}(+) / \mathrm{H}(+)$ exchange regulatory cofactor NHE-RF2 OS=Mus musculus GN=Slc9a3r2 - [NHRF2_MOUSE] & $0,000 E 0$ & $0,000 E 0$ & $0,000 \mathrm{E}_{0}$ & 2,46957 \\
\hline P62137 & Serine/threonine-protein phosphatase PP1-alpha catalytic subunit OS=Mus musculus GN=Ppp1ca PE=1 SV=1- [PP1A_MOUSE] & $0,000 \mathrm{E} 0$ & $0,000 \mathrm{E} 0$ & 2,353E6 & $8,782 \mathrm{E7}$ \\
\hline P63276 & 40 s ribosomal protein $\mathrm{S17}$ OS=Mus musculus GN=Rps17 PE=1 SV=2 - [RS17_MOUSE] & $0,000 E 0$ & $0,000 E 0$ & $0,000 \mathrm{E} 0$ & $1,737 E 8$ \\
\hline Q9R1K9 & Centrin-2 OS=Mus musculus GN=Cetn2 PE=1 SV=1 - [CETN2_MOUSE] & $0,0000_{0}$ & $0,000 \mathrm{EO}$ & $0,000 \mathrm{E}_{0}$ & $5,634 \mathrm{E} 6$ \\
\hline Q9DBJ1 & Phosphoglycerate mutase 1 OS=Mus musculus GN=Pgam1 $P E=1 \mathrm{SV}=3$ - [PGAM1_MOUSE] & $0,000 E 0$ & $0,000 E 0$ & $0,000 \mathrm{E} 0$ & $2,791 \mathrm{E}$ \\
\hline P27659 & 60S ribosomal protein L3 OS=Mus musculus GN=Rpl3 PE=1 SV=3 - [RL3_MOUSE] & $0,000 E 0$ & $0,000 E_{0}$ & 3,066E6 & 9,362E7 \\
\hline P38647 & Stress-70 protein, mitochondrial OS=Mus musculus GN=Hspa9 PE=1 SV=3 - [GRP75_MOUSE] & $6,017 E 7$ & $0,000 E 0$ & $2,130 \mathrm{E} 7$ & $2,372 \mathrm{E} 8$ \\
\hline P68510 & 14-3-3 protein eta OS=Mus musculus $\mathrm{GN}=$ Ywhah $\mathrm{PE}=1 \mathrm{SV}=2$ - [1433F_MOUSE] & $0,000 E 0$ & $0,000 E 0$ & 7,196E5 & $1,335 E 8$ \\
\hline P62242 & 40S ribosomal protein $\mathrm{S8}$ OS=Mus musculus GN=Rps8 PE=1 SV=2 - [RS8_MOUSE] & $0,000 E 0$ & $0,000 E_{0}$ & $1,222 E 6$ & $3,501 E 8$ \\
\hline P61358 & 60 ribosomal protein L27 OS=Mus musculus GN=Rp127 PE=1 SV=2 - [RL27_MOUSE] & $0,000 E 0$ & $0,000 \mathrm{E} O$ & $0,000 \mathrm{E} 0$ & $3,338 \mathrm{E} 8$ \\
\hline P08752 & Guanine nucleotide-binding protein $\mathrm{G}(\mathrm{i})$ subunit alpha-2 $\mathrm{OS}=$ Mus musculus $\mathrm{GN}=\mathrm{Gnai2}$ PE=1 $\mathrm{SV}=5$ - [GNAI2_MOUSE] & $0,000 E 0$ & $0,000 \mathrm{E} 0$ & $7,794 E 4$ & 5,374E7 \\
\hline P62869 & Elongin-B OS=Mus musculus GN=Elob PE=1 SV=1 - [ELOB_MOUSE] & $0,000 E 0$ & $0,000 \mathrm{EO}$ & $0,000 E 0$ & $1,002 E 7$ \\
\hline P54823 & Probable ATP-dependent RNA helicase DDX6 OS=Mus musculus GN=Dd×6 PE=1 SV=1 - [DDX6_MOUSE] & $0,000 E 0$ & $0,000 E 0$ & $0,000 E 0$ & 4,259E7 \\
\hline P62918 & $60 S$ ribosomal protein L8 OS=Mus musculus GN=Rpl8 PE=1 SV=2 - [RL8_MOUSE] & $0,000 E_{0}$ & $0,000 \mathrm{E} 0$ & $0,000 E_{0}$ & \begin{tabular}{l|l|l}
$1,063 \mathrm{E} 8$ \\
\end{tabular} \\
\hline Q8BKX1 & Brain-specific angiogenesis inhibitor 1-associated protein 2 OS=Mus musculus GN=Baiap2 PE=1 SV=2 - [BAIP2_MOUSE] & $0,000 \mathrm{E} 0$ & $0,000 \mathrm{E} 0$ & $0,000 \mathrm{E} 0$ & $1,514 \mathrm{E}$ \\
\hline 088569 & Heterogeneous nuclear ribonucleoproteins $\mathrm{A2} / \mathrm{B} 1 \mathrm{OS}=$ Mus musculus $\mathrm{GN}=\mathrm{Hnrnpa2b1} \mathrm{PE}=1 \mathrm{SV}=2$ - [ROA2_MOUSE] & $0,000 E 0$ & $0,000 \mathrm{E} 0$ & $0,000 \mathrm{E}_{0}$ & \begin{tabular}{l|l}
$4,976 \mathrm{E} 7$ \\
\end{tabular} \\
\hline 009044 & Synaptosomal-associated protein $23 \mathrm{OS}=$ Mus musculus GN=Snap23 PE=1 SV=1 - [SNP23_MOUSE] & $0,000 E 0$ & $0,000 E 0$ & $0,000 E_{0}$ & $1,280 \mathrm{E} 7$ \\
\hline P61205 & ADP-ribosylation factor 3 OS=Mus musculus GN=Ar3 PE=2 SV=2 - [ARF3_MOUSE] & $0,0000_{0}$ & $0,000 \mathrm{E} 0$ & $0,0000_{0}$ & 5,665E7 \\
\hline Q02248 & Catenin beta-1 OS=Mus musculus GN=Ctnnb1 PE=1 SV=1 - [CTNB1_MOUSE] & $0,000 E 0$ & $0,000 \mathrm{E}$ & 3,246E 6 & $3,082 E 8$ \\
\hline Q5SYDO & Unconventional myosin-Id OS=Mus musculus GN=Myo1d PE=1 SV=1 - [MYO1D_MOUSE] & $2,735 \mathrm{E} 6$ & $0,000 \mathrm{E} 0$ & \begin{tabular}{r|r|}
$6,818 \mathrm{E} 6$ \\
\end{tabular} & $1,427 \mathrm{E} 8$ \\
\hline P62281 & 40 ribosomal protein $\mathrm{S} 11$ OS=Mus musculus GN=Rps11 PE=1 SV=3 - [RS11_MOUSE] & $0,000 E 0$ & $0,000 E 0$ & $4,261 E 6$ & $1,330 \mathrm{E} 8$ \\
\hline Q64523 & Histone H2A type 2-C OS=Mus musculus GN=Hist2h2ac PE=1 SV=3 - [H2A2C_MOUSE] & $0,000 E 0$ & $0,000 E 0$ & $1,828 \mathrm{E}$ & 4,055E7 \\
\hline P83940 & Elongin-C OS=Mus musculus $\mathrm{GN}=E$ Eloc PE=1 SV=1 - [ELOC_MOUSE] & $0,000 E 0$ & $0,000 E_{0}$ & $0,000 \mathrm{E}_{0}$ & $1,346 \mathrm{E} 7$ \\
\hline P29391 & Ferritin light chain 1 OS=Mus musculus GN=Ft11 PE=1 SV=2 - [FRIL1_MOUSE] & $0,000 E 0$ & $0,000 E_{0}$ & $0,000 \mathrm{E} 0$ & $2,343 E 7$ \\
\hline P97855 & Ras GTPase-activating protein-binding protein 1 OS=Mus musculus GN=G3bp1 PE=1 SV=1-[G3BP1_MOUSE] & $8,322 E 6$ & $0,000 \mathrm{E} 0$ & $0,000 E_{0}$ & $1,364 E 8$ \\
\hline P68037 & Ubiquitin-conjugating enzyme E2 L3 OS=Mus musculus GN=Ube213 PE=1 SV=1 - [UB2L3_MOUSE] & $0,000 E 0$ & $0,000 E 0$ & $0,000 E 0$ & $8,416 \mathrm{E} 6$ \\
\hline Q9D8E6 & $60 S$ ribosomal protein L4 OS=Mus musculus GN=Rpl4 PE=1 SV=3 - [RL4_MOUSE] & $0,000 E 0$ & $0,000 E 0$ & $1,238 E 6$ & 2,018E8 \\
\hline P68372 & Tubulin beta-4B chain OS=Mus musculus GN=Tubb4b PE=1 $\mathrm{SV}=1-\left[\mathrm{TBB} 4 \mathrm{~B} \_\right.$MOUSE] & 7,924E6 & $0,000 E 0$ & $5,484 E 6$ & $1,362 E 8$ \\
\hline Q8BT07 & Centrosomal protein of $55 \mathrm{kDa}$ OS=Mus musculus GN=Cep55 PE=1 $1 \mathrm{SV}=2$ - [CEP55_MOUSE] & $0,000 \mathrm{EO}^{2}$ & $0,000 \mathrm{E} 0$ & $0,000 \mathrm{E} 0$ & 7,690E7 \\
\hline P17426-2 & Isoform B of AP-2 complex subunit alpha-1 OS=Mus musculus GN=AP2a1 - [AP2A1_MOUSE] & $0,000 E 0$ & $0,000 \mathrm{E} 0$ & $0,000 \mathrm{E}_{0}$ & \begin{tabular}{l|l}
$1,427 \mathrm{E} 8$ \\
\end{tabular} \\
\hline P98078 & Disabled homolog 2 OS=Mus musculus GN=Dab2 PE=1 SV=2 - [DAB2_MOUSE] & $0,000 E 0$ & $0,000 E 0$ & $0,000 E_{0}$ & $2,629 \mathrm{E}$ \\
\hline P12970 & $60 S$ ribosomal protein L7a OS=Mus musculus GN=Rpl7a PE=1 SV=2 - [RL7A_MOUSE] & $0,000 E 0$ & $0,000 E 0$ & $9,615 E 5$ & $2,438 E 8$ \\
\hline P62983 & Ubiquitin-40S ribosomal protein S27a OS=Mus musculus GN=Rps27a PE=1 SV=2 - [RS27A_MOUSE] & $2,631 \mathrm{E}$ & $0,000 E 0$ & $2,882 E 7$ & $7,431 E 8$ \\
\hline Q8BFZ3 & Beta-actin-like protein 2 OS=Mus musculus GN=Actb12 PE=1 SV=1 - [ACTBL_MOUSE] & $6,609 E 8$ & $0,000 E 0$ & 1,502E8 & $5,101 \mathrm{Eg}$ \\
\hline Q9CY58-2 & Isoform 2 of Plasminogen activator inhibitor 1 RNA-binding protein OS=Mus musculus GN=Serbp1 - [PAIRB_MOUSE] & $0,000 E 0$ & $0,000 \mathrm{E} 0$ & \begin{tabular}{l|l}
$1,047 \mathrm{E}$ \\
\end{tabular} & $1,214 \mathrm{E} 8$ \\
\hline P20029 & 78 kDa glucose-regulated protein OS=Mus musculus GN=Hspa5 PE=1 SV=3 - [GRP78_MOUSE] & $6,100 \mathrm{E} 7$ & $0,000 \mathrm{E} 0$ & $1,220 \mathrm{E} 7$ & $1,944 \mathrm{E} 8$ \\
\hline Q61584-3 & Isoform B of Fragile X mental retardation syndrome-related protein 1 OS=Mus musculus GN=Fxr1 - [FXR1_MOUSE] & $0,000 E 0$ & $0,000 E_{0}$ & $3,780 \mathrm{E} 5$ & $9,430 \mathrm{E} 7$ \\
\hline P62960 & Nuclease-sensitive element-binding protein 1 OS=Mus musculus $\mathrm{GN}=\mathrm{Ybx} 1 \mathrm{PE}=1 \mathrm{SV}=3$ - [YBOX1_MOUSE] & $1,609 E 7$ & $0,000 E 0$ & $8,242 E 5$ & $2,920 E 8$ \\
\hline P61161 & Actin-related protein 2 OS=Mus musculus GN=Actr2 PE=1 SV=1 - [ARP2_MOUSE] & $0,000 E 0$ & $0,000 E_{0}$ & 2,837E6 & $1,061 E 8$ \\
\hline P68033 & Actin, alpha cardiac muscle 1 OS=Mus musculus GN=Actc1 PE=1 $1 \mathrm{SV}=1$ - [ACTC_MOUSE] & 7,491E8 & $0,000 \mathrm{E}^{2}$ & $1,763 \mathrm{E} 8$ & 9,328E9 \\
\hline Q9CQ19 & Myosin regulatory light polypeptide 9 OS=Mus musculus GN=Myl9 PE=1 SV=3 - [MYL9_MOUSE] & 1,440E8 & $0,000 E 0$ & $1,331 \mathrm{E} 8$ & $5,016 \mathrm{Eg}$ \\
\hline P67871 & Casein kinase II subunit beta OS=Mus musculus GN=Csnk2b PE=1 SV=1 - [CSK2B_MOUSE] & $0,000 E 0$ & $0,000 E 0$ & $0,000 \mathrm{E}_{0}$ & $\begin{array}{ll}8,717 \mathrm{E} 6 \\
\end{array}$ \\
\hline P80318 & T-complex protein 1 subunit gamma $\mathrm{OS}=$ Mus musculus $\mathrm{GN}=\mathrm{Cct} 3 \mathrm{PE}=1 \mathrm{SV}=1$ - [TCPG_MOUSE] & $0,000 E 0$ & $0,000 E 0$ & $0,000 E_{0}$ & $2,080 \mathrm{E} 7$ \\
\hline P14206 & $40 S$ ribosomal protein SA OS=Mus musculus GN=Rpsa PE=1 SV=4 - [RSSA_MOUSE] & $0,000 \mathrm{E} 0$ & $0,000 \mathrm{E} 0$ & $0,000 \mathrm{E} 0$ & \begin{tabular}{l|l|l}
$4,752 E 8$ & \\
\end{tabular} \\
\hline P29341 & Polyadenylate-binding protein 1 OS=Mus musculus GN=Pabpc1 PE=1 SV=2 - [PABP1_MOUSE] & $0,000 E 0$ & $0,000 \mathrm{E} 0$ & $0,000 \mathrm{E} 0$ & $1,375 E 8$ \\
\hline Q9JHL1 & $\mathrm{Na}(+) / \mathrm{H}(+)$ exchange regulatory cofactor NHE-RF2 OS=Mus musculus GN=Slc9a3r2 PE=1 $\mathrm{SV}=2$ - [NHRF2_MOUSE] & $0,000 E 0$ & $0,000 \mathrm{E} 0$ & $0,000 \mathrm{E}_{0}$ & $2,469 \mathrm{E} 7$ \\
\hline P62751 & 60 ribosomal protein L23a OS=Mus musculus GN=Rpl23a PE=1 SV=1 - [RL23A_MOUSE] & $0,000 E 0$ & $0,000 E 0$ & $1,154 \mathrm{E}$ & $2,928 E 8$ \\
\hline P51881 & ADP/ATP translocase 2 OS=Mus musculus GN=SIc25a5 PE=1 SV=3 - [ADT2_MOUSE] & $3,742 E 5$ & $0,000 E 0$ & 1,161E7 & $1,170 \mathrm{E}$ \\
\hline Q61033 & Lamina-associated polypeptide 2, isoforms alpha/zeta OS=Mus musculus GN=Tmpo PE=1 $\mathrm{SV}=4$ - [LAP2A_MOUSE] & $0,0000_{0}$ & $0,000 E 0$ & 7,435E5 & $8,448 E 7$ \\
\hline Q9D8B3 & Charged multivesicular body protein $4 \mathrm{~b}$ OS=Mus musculus GN=Chmp4b PE=1 SV=2 - [CHM4B_MOUSE] & $0,000 \mathrm{E} 0$ & $0,000 \mathrm{E} 0$ & $0,000 \mathrm{E} 0$ & \begin{tabular}{l|l|l}
$1,016 \mathrm{E} 8$ \\
\end{tabular} \\
\hline P62301 & 40S ribosomal protein $\mathrm{S} 13$ OS=Mus musculus GN=Rps13 PE=1 SV=2 - [RS13_MOUSE] & $0,000 \mathrm{E} 0$ & $0,000 \mathrm{E} 0$ & 3,325E6 & $1,528 \mathrm{E} 8$ \\
\hline P99024 & Tubulin beta-5 chain OS=Mus musculus GN=Tubb5 PE=1 SV=1 - [TBB5_MOUSE] & 7,924E6 & $0,000 \mathrm{E} 0$ & $5,484 E 6$ & $1,705 E 8$ \\
\hline P84104-2 & Isoform Short of Serine/arginine-rich splicing factor 3 OS=Mus musculus GN=Srsf3 - [SRSF3_MOUSE] & $0,000 E 0$ & $0,000 E 0$ & $0,000 E_{0}$ & 6,099E7 \\
\hline Q99K01 & Pyridoxal-dependent decarboxylase domain-containing protein 1 OS=Mus musculus GN=Pdxdc1 PE=1 SV=2 - [PDXD1_MOUSE] & $0,000 E 0$ & $0,000 \mathrm{EO}$ & $0,000 E 0$ & $1,205 E 8$ \\
\hline P63325 & $40 S$ ribosomal protein $\mathrm{S} 10$ OS=Mus musculus GN=Rps10 PE=1 SV=1 - [RS10_MOUSE] & $0,000 E_{0}$ & $0,000 \mathrm{E} 0$ & $0,000 \mathrm{E} 0$ & $1,503 \mathrm{E} 8$ \\
\hline P62141 & Serine/threonine-protein phosphatase PP1-beta catalytic subunit OS=Mus musculus GN=Ppp1cb PE=1 SV=3 - [PP1B_MOUSE] & $0,000 E 0$ & $0,000 E 0$ & 2,353E6 & $5,939 \mathrm{E} 7$ \\
\hline P47911 & 605 ribosomal protein L6 OS=Mus musculus GN=Rpl6 PE=1 SV=3 - [RL6_MOUSE] & $0,000 E 0$ & $0,000 E 0$ & $2,927 E 6$ & $1,822 E 8$ \\
\hline Q99K01-2 & Isoform 2 of Pyridoxal-dependent decarboxylase domain-containing protein 1 OS=Mus musculus GN=Pdxdc1 - [PDXD1_MOUSE] & $0,000 E 0$ & $0,000 \mathrm{E} 0$ & $0,000 \mathrm{E}_{0}$ & $1,547 \boxminus 8$ \\
\hline P17742 & Peptidyl-prolyl cis-trans isomerase A OS=Mus musculus GN=Ppia PE=1 SV=2 - [PPIA_MOUSE] & $0,000 E 0$ & $0,000 E 0$ & 9,673E5 & $1,240 \mathrm{E} 8$ \\
\hline Q6ZWN5 & 40 r ribosomal protein 59 OS=Mus musculus GN=Rps9 PE=1 SV=3 - [RS9_MOUSE] & $0,000 E 0$ & $0,000 E 0$ & $4,221 E 6$ & $1,742 E 8$ \\
\hline Q30056 & Ras-interacting protein 1 OS=Mus musculus GN=Rasip1 PE=1 SV=3 - [RAIN_MOUSE] & $5,784 E 6$ & $0,000 E_{0}$ & 1,974E6 & $2,222 E 8$ \\
\hline P68373 & Tubulin alpha-1C chain OS=Mus musculus GN=Tuba1c PE=1 SV=1 - [TBA1C_MOUSE] & $1,425 \mathrm{E} 7$ & $0,000 \mathrm{E} 0$ & $\begin{array}{ll}7,585 E 6 \\
\end{array}$ & 7,937E7 \\
\hline P63037 & Dna] homolog subfamily A member 1 OS=Mus musculus GN=Dnaja1 PE=1 SV=1 - [DNJA1_MOUSE] & $0,0000_{0}$ & $0,000 \mathrm{E} 0$ & $0,000 \mathrm{E}_{0}$ & $2,998 E 7$ \\
\hline Q9JKB3 & Y-box-binding protein 3 OS=Mus musculus GN=Ybx3 PE=1 SV=2 - [YBOX__MOUSE] & $0,000 E 0$ & $0,000 E 0$ & $0,000 E_{0}$ & $1,701 E 8$ \\
\hline Q60598 & Src substrate cortactin OS=Mus musculus $\mathrm{GN}=\mathrm{Cttn} \mathrm{PE}=1 \mathrm{SV}=2$ - [SRC8_MOUSE] & $0,000 E 0$ & $0,000 E 0$ & $0,000 E_{0}$ & 9,379E7 \\
\hline P07356 & Annexin A2 OS=Mus musculus GN=Anxa2 PE=1 SV=2 - [ANXA2_MOUSE] & $0,000 E 0$ & $0,000 E 0$ & $1,418 E 6$ & $4,466 \mathrm{E} 7$ \\
\hline Q9נJ18 & $60 S$ ribosomal protein $L 38$ OS=Mus musculus GN=Rp|38 PE=1 SV=3 - [RL38_MOUSE] & $0,000 E 0$ & $0,000 E 0$ & $0,000 \mathrm{E} 0$ & 6,069E7 \\
\hline Q8K371 & Angiomotin-like protein 2 OS=Mus musculus GN=Amot12 PE=1 SV=1 - [AMOL2_MOUSE] & $0,000 E 0$ & $0,000 \mathrm{E} 0$ & $0,000 E 0$ & 2,745E8 \\
\hline P10126 & Elongation factor 1-alpha 1 OS=Mus musculus GN=Eef1a1 PE=1 SV=3 - [EF1A1_MOUSE] & $4,552 \mathrm{E} 6$ & $0,000 \mathrm{E} 0$ & $2,176 \mathrm{E} 6$ & $\begin{array}{ll}4,168 \mathrm{E} 8 \\
\end{array}$ \\
\hline Q9D1G1 & Ras-related protein Rab-1B OS=Mus musculus GN=Rab1b PE=1 SV=1 - [RAB1B_MOUSE] & $0,000 E 0$ & $0,000 E 0$ & $3,456 \mathrm{E} 5$ & 6,625E7 \\
\hline P42208 & Septin-2 OS=Mus musculus GN=Sept2 PE=1 SV=2 - [SEPT2_MOUSE] & $0,000 E 0$ & $0,000 \mathrm{E} 0$ & $0,000 \mathrm{E} 0$ & $2,179 E 7$ \\
\hline Q9נJ28 & Protein flightless-1 homolog OS=Mus musculus GN=Flii PE=1 SV=1 - [FLI_MOUSE] & $0,000 E 0$ & $0,000 \mathrm{EO}$ & $0,000 \mathrm{E}^{-}$ & $1,469 E 8$ \\
\hline Q99104 & Unconventional myosin-Va OS=Mus musculus GN=Myo5a PE=1 SV=2 - [MYO5A_MOUSE] & $2,735 E 6$ & $0,000 E 0$ & 6,683E5 & $8,479 E 7$ \\
\hline Q9DBG3 & AP-2 complex subunit beta OS=Mus musculus GN=Ap2b1 PE=1SV=1 - [AP2B1_MOUSE] & $0,000 E 0$ & $0,000 E_{0}$ & $0,000 \mathrm{E} 0$ & $2,544 \mathrm{E} 8$ \\
\hline P63323 & $40 S$ ribosomal protein $\mathrm{S12}$ OS=Mus musculus GN=Rps12 PE=1 SV=2 - [RS12_MOUSE] & $0,000 E 0$ & $0,000 E 0$ & $1,701 E 5$ & $1,409 \mathrm{E} 8$ \\
\hline Q8K4L3-3 & Isoform 2 of Supervillin OS=Mus musculus GN=Svil - [SVIL_MOUSE] & $0,000 E 0$ & $0,000 E 0$ & $4,058 E 5$ & 9,909E7 \\
\hline P62858 & $40 S$ ribosomal protein $S 28$ OS=Mus musculus GN=Rps28 PE=1 SV=1 - [RS28_MOUSE] & $1,014 \mathrm{E}$ & $0,000 E 0$ & $9,284 E 6$ & $5,536 \mathrm{E} 7$ \\
\hline Q4ACU6 & SH3 and multiple ankyrin repeat domains protein 3 OS=Mus musculus $\mathrm{GN}=$ Shank3 PE=1 SV=3 - [SHAN3_MOUSE] & $0,000 E 0$ & $0,000 E 0$ & $0,000 \mathrm{E} 0$ & 7,759E7 \\
\hline Q80X90 & Filamin-B OS=Mus musculus GN=Finb PE=1 SV=3 - [FLNB_MOUSE] & $0,000 E 0$ & $0,000 E 0$ & $1,416 \mathrm{E} 6$ & $3,471 \mathrm{E}$ \\
\hline Q99LF4 & tRNA-splicing ligase RtcB homolog OS=Mus musculus $\mathrm{GN}=\mathrm{Rtcb} P E=1 \mathrm{SV}=1$ - [RTCB_MOUSE] & $0,0000_{0}$ & $0,000 E_{0}$ & $0,000 \mathrm{E}_{0}$ & $2,398 \mathrm{E}$ \\
\hline Q88ZQ9 & Eukaryotic translation initiation factor 3 subunit $B$ OS=Mus musculus GN=Eif3b PE=1 SV=1- [EFF3B_MOUSE] & $0,000 E 0$ & $0,000 E 0$ & $0,000 E_{0}$ & $3,702 E 7$ \\
\hline P68134 & Actin, alpha skeletal muscle OS=Mus musculus GN=Acta1 PE=1 SV=1 - [ACTS_MOUSE] & 7,491E8 & $0,000 E 0$ & 1,507E8 & 9,328E9 \\
\hline Q60737 & Casein kinase II subunit alpha OS=Mus musculus $\mathrm{GN}=C$ Csnk2a1 PE $=1 \mathrm{SV}=2$ - [CSK21_MOUSE] & $0,000 E 0$ & $0,000 E 0$ & $0,000 E 0$ & $3,069 \mathrm{E} 7$ \\
\hline
\end{tabular}




\begin{tabular}{|c|c|c|}
\hline P53026 & 60 S ribosomal protein L10a OS=Mus musculus GN=Rpl10a PE=1 SV=3 - [RL10A_MOUSE] & $0,000 \mathrm{E} 0$ \\
\hline Q9CZM2 & 60S ribosomal protein L15 OS=Mus musculus GN=Rpl15 PE=2 SV=4 - [RL15_MOUSE] & $0,000 E 0$ \\
\hline Q9JMH9 & Unconventional myosin-XVIIIa OS=Mus musculus GN=Myo18a PE=1 SV=2 - [MY18A_MOUSE] & $0,000 E 0$ \\
\hline Q04736 & Tyrosine-protein kinase Yes OS=Mus musculus GN=Yes1 PE=1 SV=3 - [YES_MOUSE] & $0,000 E 0$ \\
\hline P60867 & 40 r ribosomal protein S20 OS=Mus musculus GN=Rps20 PE=1 SV=1 - [RS20_MOUSE] & $0,000 E 0$ \\
\hline Q3UZ39 & Leucine-rich repeat flightless-interacting protein 1 OS=Mus musculus GN=Lrrfip1 PE=1SV=2 - [LRRF1_MOUSE] & $0,000 E 0$ \\
\hline P62843 & 40S ribosomal protein S15 OS=Mus musculus GN=Rps15 PE=1 SV=2 - [RS15_MOUSE] & $0,000 E 0$ \\
\hline Q8BP67 & 60S ribosomal protein L24 OS=Mus musculus GN=Rpl24 PE=1 SV=2 - [RL24_MOUSE] & $0,000 E 0$ \\
\hline 055142 & 60S ribosomal protein L35a OS=Mus musculus GN=Rpl35a PE=1 SV=2 - [RL35A_MOUSE] & $0,000 E 0$ \\
\hline Q9Z0F4 & Calcium and integrin-binding protein 1 OS=Mus musculus GN=Cib1 PE=1 SV=3 - [CIB1_MOUSE] & $0,000 \mathrm{E} 0$ \\
\hline Q64331 & Unconventional myosin-VI OS=Mus musculus GN=Myo6 PE=1 SV=1 - [MYO6_MOUSE] & $0,000 E 0$ \\
\hline Q61584-7 & Isoform G of Fragile X mental retardation syndrome-related protein 1 OS=Mus musculus GN=Fxr1 - [FXR1_MOUSE] & $0,000 \mathrm{EO}_{0}$ \\
\hline P84084 & ADP-ribosylation factor 5 OS $=$ Mus musculus $\mathrm{GN}=\mathrm{ArF} 5 \mathrm{PE}=1 \mathrm{SV}=2$ - [ARF5_MOUSE] & $0,000 E 0$ \\
\hline Q99kW3-1 & Isoform 1 of TRIO and F-actin-binding protein OS=Mus musculus GN=Triobp - [TARA_MOUSE] & $0,000 E 0$ \\
\hline P25444 & $40 S$ ribosomal protein S2 OS=Mus musculus GN=Rps2 PE=1 SV=3 - [RS2_MOUSE] & $0,000 \mathrm{EO}$ \\
\hline Q9IIW9 & Ras-related protein Ral-B OS=Mus musculus GN=Ralb PE=1SV=1-[RALB_MOUSE] & $0,000 E 0$ \\
\hline P11499 & Heat shock protein HSP 90-beta OS=Mus musculus GN=Hsp90ab1 PE=1 SV=3 - [HS90B_MOUSE] & $0,000 \mathrm{EO}$ \\
\hline P62821 & Ras-related protein Rab-1A OS=Mus musculus GN=Rab1A PE=1 SV=3 - [RAB1A_MOUSE] & $0,000 E 0$ \\
\hline P09528 & Ferritin heavy chain OS=Mus musculus GN=Fth1 PE=1 SV=2 - [FRIH_MOUSE] & $0,000 E 0$ \\
\hline P68368 & Tubulin alpha-4A chain OS=Mus musculus GN=Tuba4a PE=1 SV=1 - [TBA4A_MOUSE] & $1,425 \mathrm{E} 7$ \\
\hline Q61599 & Rho GDP-dissociation inhibitor 2 OS=Mus musculus GN=Arhgdib PE=1 SV=3 - [GDIR2_MOUSE] & $0,000 E 0$ \\
\hline Q9CQR2 & 40S ribosomal protein S21 OS=Mus musculus GN=Rps21 PE=1 SV=1 - [RS21_MOUSE] & $0,000 E 0$ \\
\hline P19253 & 60 S ribosomal protein L13a OS=Mus musculus GN=Rpl13a PE=1 SV=4 - [RL13A_MOUSE] & $0,000 E 0$ \\
\hline Q9WUM4 & Coronin-1C OS=Mus musculus GN=Coro1c PE=1 SV=2 - [COR1C_MOUSE] & $0,000 E 0$ \\
\hline P80317 & T-complex protein 1 subunit zeta OS=Mus musculus GN=Cct6a PE=1 SV=3 - [TCPZ_MOUSE] & $0,000 E 0$ \\
\hline Q5FWH6 & Rho guanine nucleotide exchange factor 15 OS=Mus musculus GN=Arhgef15 PE=1 SV=1 - [ARHGF_MOUSE] & $0,000 E 0$ \\
\hline P99029-2 & Isoform Cytoplasmic+peroxisomal of Peroxiredoxin-5, mitochondrial OS=Mus musculus GN=Prdx5 - [PRDX5_MOUSE] & $0,000 E 0$ \\
\hline Q8BMK4 & Cytoskeleton-associated protein 4 OS=Mus musculus GN=Ckap4 $\mathrm{PE}=1 \mathrm{SV}=2$ - [CKAP4_MOUSE] & $0,000 \mathrm{EO}_{0}$ \\
\hline Q4ACU6-10 & Isoform 9 of SH3 and multiple ankyrin repeat domains protein 3 OS=Mus musculus GN=Shank3 - [SHAN3_MOUSE] & $0,000 E 0$ \\
\hline Q922F4 & Tubulin beta-6 chain OS=Mus musculus GN=Tubb6 PE=1 SV=1 - [TBB6_MOUSE] & $0,000 E 0$ \\
\hline Q7TPV4 & Myb-binding protein $1 \mathrm{~A}$ OS=Mus musculus GN=Mybbp1a PE=1 SV=2 - [MBB1A_MOUSE] & $0,000 E 0$ \\
\hline P09411 & Phosphoglycerate kinase $10 \mathrm{OS}=$ Mus musculus GN=Pgk1 PE=1 SV=4 - [PGK1_MOUSE] & $0,000 \mathrm{EO}$ \\
\hline 009167 & 60S ribosomal protein L21 OS=Mus musculus GN=Rpl21 PE=1 SV=3 - [RL21_MOUSE] & $0,000 \mathrm{E} 0$ \\
\hline Q02257 & Junction plakoglobin OS=Mus musculus GN=Jup PE=1 SV=3 - [PLAK_MOUSE] & $0,000 E 0$ \\
\hline Q8BX10 & Serine/threonine-protein phosphatase PGAM5, mitochondrial OS=Mus musculus GN=Pgam5 PE=1 SV=1 - [PGAM5_MOUSE] & $0,000 E 0$ \\
\hline Q63918 & Serum deprivation-response protein OS=Mus musculus GN=Sdpr PE=1 SV=3 - [SDPR_MOUSE] & $0,000 E 0$ \\
\hline Q8BX10-2 & Isoform 2 of Serine/threonine-protein phosphatase PGAM5, mitochondrial OS=Mus musculus GN=Pgam5 - [PGAM5_MOUSE] & $0,000 E 0$ \\
\hline Q99PL5 & Ribosome-binding protein 1 OS=Mus musculus GN=Rrbp1 PE=1 SV=2 - [RRBP1_MOUSE] & $0,000 E 0$ \\
\hline Q6PDM2 & Serine/arginine-rich splicing factor 1 OS=Mus musculus GN=Srsf1 PE=1 SV=3 - [SRSF1_MOUSE] & $0,000 E 0$ \\
\hline Q9CQ69 & Cytochrome b-c1 complex subunit 8 OS=Mus musculus GN=Uqcra PE=1 SV=3 - [QCR8_MOUSE] & $0,000 E_{0}$ \\
\hline Q64152 & Transcription factor BTF3 OS=Mus musculus GN=Btr3 PE=1 SV=3 - [BTF3_MOUSE] & $0,000 E 0$ \\
\hline Q9ROP3 & S-formylglutathione hydrolase OS=Mus musculus GN=Esd PE=1 SV=1 - [ESTD_MOUSE] & $0,000 E 0$ \\
\hline Q01853 & Transitional endoplasmic reticulum ATPase OS=Mus musculus GN=VCP PE=1 SV=4 - [TERA_MOUSE] & $0,000 \mathrm{EO}$ \\
\hline Q9JJL8 & Serine--tRNA ligase, mitochondrial OS=Mus musculus GN=Sars2 PE=1 SV=2 - [SYSM_MOUSE] & $0,000 E 0$ \\
\hline P06151 & L-lactate dehydrogenase A chain OS=Mus musculus GN=Ldha PE=1 SV=3 - [LDHA_MOUSE] & $0,000 E 0$ \\
\hline QTTSJ2-3 & Isoform 3 of Microtubule-associated protein 6 OS=Mus musculus GN=Map6 - [MAP6_MOUSE] & $0,000 E 0$ \\
\hline Q9ERG0 & LIM domain and actin-binding protein 1 OS=Mus musculus GN=Lima1 PE=1 SV=3 - [LMA1_MOUSE] & $0,000 E 0$ \\
\hline Q7TMM9 & Tubulin beta-2A chain OS=Mus musculus GN=Tubb2a PE=1 SV=1 - [TBB2A_MOUSE] & 7,924E6 \\
\hline 054724 & Polymerase I and transcript release factor OS=Mus musculus GN=Ptrf PE=1 SV=1 - [PTRF_MOUSE] & $0,000 E 0$ \\
\hline P62880 & Guanine nucleotide-binding protein $\mathrm{G}(\mathrm{I}) / \mathrm{G}(\mathrm{S}) / \mathrm{G}(\mathrm{T})$ subunit beta-2 $\mathrm{OS}=$ Mus musculus $\mathrm{GN}=\mathrm{Gnb2} \mathrm{PE}=1 \mathrm{SV}=3$ - [GBB2_MOUSE] & $0,000 \mathrm{EO}$ \\
\hline 054931-2 & Isoform 2 of A-kinase anchor protein 2 OS=Mus musculus GN=Akap2 - [AKAP2_MOUSE] & $0,000 E 0$ \\
\hline P05064 & Fructose-bisphosphate aldolase A OS=Mus musculus GN=Aldoa PE=1 SV=2 - [ALDOA_MOUSE] & $0,000 E 0$ \\
\hline Q8K4L3 & Supervilin OS=Mus musculus GN=Svil PE=1 SV=1 - [SVIL_MOUSE] & $0,000 E 0$ \\
\hline Q9JKF1 & Ras GTPase-activating-like protein IQGAP1 OS=Mus musculus GN=Igaap1 PE=1 SV=2 - [IQGA1_MOUSE] & $0,000 E 0$ \\
\hline Q8KODO & Cyclin-dependent kinase 17 OS=Mus musculus GN=Cdk17 PE=1 SV=2 - [CDK17_MOUSE] & $0,000 \mathrm{E} 0$ \\
\hline P70372 & ELAV-like protein 1 OS=Mus musculus GN=Elav11 PE=1 SV=2 - [ELAV1_MOUSE] & $0,000 E 0$ \\
\hline F8VQB6 & Unconventional myosin-X OS=Mus musculus GN=Myo10 PE=1 SV=1 - [MYO10_MOUSE] & $0,000 E 0$ \\
\hline Q91YR1 & Twinflin-1 OS=Mus musculus GN=Twf1 PE=1 SV=2 - [TWF1_MOUSE] & $0,000 E 0$ \\
\hline P58774-2 & Isoform 2 of Tropomyosin beta chain OS=Mus musculus GN=Tpm2 - [TPM2_MOUSE] & $0,000 E 0$ \\
\hline P17427 & AP-2 complex subunit alpha-2 OS=Mus musculus GN=AP2a2 PE=1 SV=2 - [AP2A2_MOUSE] & $0,000 \mathrm{EO}$ \\
\hline Q61553 & Fascin OS=Mus musculus GN=Fscn1 PE=1 $\mathrm{SV}=4$ - [FSCN1_MOUSE] & $0,000 E 0$ \\
\hline Q8VD75 & Huntingtin-interacting protein 1 OS=Mus musculus GN=Hip1 PE=1 SV=2 - [HIP1_MOUSE] & $0,000 E 0$ \\
\hline Q9CY58 & Plasminogen activator inhibitor 1 RNA-binding protein OS=Mus musculus GN=Serbp1 PE=1 $1 \mathrm{SV}=2$ - [PAIRB_MOUSE] & $0,000 E 0$ \\
\hline Q62036 & Centrosomal protein of $131 \mathrm{kDa}$ OS=Mus musculus GN=Cep131 PE=1 SV=2 - [CP131_MOUSE] & $0,000 E 0$ \\
\hline Q8CFIO-3 & Isoform 3 of E3 ubiquitin-protein ligase NEDD4-like OS=Mus musculus GN=Nedd4I - [NED4L_MOUSE] & $0,000 E 0$ \\
\hline Q9D0E1-2 & Isoform 2 of Heterogeneous nuclear ribonucleoprotein M OS=Mus musculus GN=Hnrnpm - [HNRPM_MOUSE] & $0,000 E 0$ \\
\hline P84096 & Rho-related GTP-binding protein RhoG OS=Mus musculus GN=Rhog PE=1 SV=1 - [RHOG_MOUSE] & $0,000 E 0$ \\
\hline Q62419 & Endophilin-A2 OS=Mus musculus GN=Sh3gl1 PE=1 SV=1 - [SH3G1_MOUSE] & $0,000 \mathrm{EO}$ \\
\hline 008583 & THO complex subunit 4 OS=Mus musculus GN=Alyref PE=1 SV=3 - [THOC4_MOUSE] & $0,000 E 0$ \\
\hline Q9DAS9 & Guanine nucleotide-binding protein $G(\mathrm{I} / \mathrm{G}(\mathrm{S}) / \mathrm{G}(\mathrm{O})$ subunit gamma-12 OS=Mus musculus $\mathrm{GN}=\mathrm{Gng} 12$ PE=1 $\mathrm{SV}=3$ - [GBG12_MOUSE] & $0,000 E 0$ \\
\hline P47962 & $60 S$ ribosomal protein L5 OS=Mus musculus GN=Rpl5 PE=1 SV=3 - [RL5_MOUSE] & $0,000 E 0$ \\
\hline 035887 & Calumenin OS=Mus musculus GN=Calu PE=1 SV=1 - [CALU_MOUSE] & $0,000 E 0$ \\
\hline P48962 & ADP/ATP translocase 1 OS=Mus musculus GN=SIc25a4 PE=1 SV=4 - [ADT1_MOUSE] & $3,742 E 5$ \\
\hline P62900 & 60S ribosomal protein L31 OS=Mus musculus GN=Rpl31 PE=1 SV=1 - [RL31_MOUSE] & $0,000 E 0$ \\
\hline P47963 & 60S ribosomal protein L13 OS=Mus musculus GN=Rpl13 PE=1 SV=3 - [RL13_MOUSE] & $0,000 E 0$ \\
\hline Q9CYL5 & Golgi-associated plant pathogenesis-related protein 1 OS=Mus musculus GN=Glipr2 PE=1 SV=3 - [GAPR1_MOUSE] & $0,000 E 0$ \\
\hline Q90757-2 & Isoform 2 of 60 r ribosomal protein L22-like 1 OS=Mus musculus GN=Rpl2211 - [RL22L_MOUSE] & $0,000 E 0$ \\
\hline P70333 & Heterogeneous nuclear ribonucleoprotein $\mathrm{H} 2 \mathrm{OS}=$ Mus musculus GN=Hnrnph2 PE=1 SV=1 - [HNRH2_MOUSE] & $0,000 E 0$ \\
\hline Q9QYB5-2 & Isoform 1 of Gamma-adducin OS=Mus musculus GN=Add3 - [ADDG_MOUSE] & $0,000 E 0$ \\
\hline P80315 & T-complex protein 1 subunit delta $\mathrm{OS}=$ Mus musculus GN=Cct4 PE=1 SV=3 - [TCPD_MOUSE] & $0,000 E 0$ \\
\hline P16045 & Galectin-1 OS=Mus musculus GN=Lgals1 PE=1 SV=3 - [LEG1_MOUSE] & $0,000 E 0$ \\
\hline Q6ZWX6 & Eukaryotic translation initiation factor 2 subunit 1 OS=Mus musculus GN=Eif2s1 PE $=1$ SV=3 - [IF2A_MOUSE] & $0,000 E 0$ \\
\hline Q88TM8 & Filamin-A OS=Mus musculus GN=Flna PE=1 SV=5 - [FLNA_MOUSE] & $0,000 \mathrm{EO}_{0}$ \\
\hline Q8VHX6-2 & Isoform 2 of Filamin-C OS=Mus musculus GN=Finc - [FLNC_MOUSE] & $0,000 E 0$ \\
\hline 035658 & Complement component 1 Q subcomponent-binding protein, mitochondrial OS=Mus musculus GN=C1qbp PE=1SV=1- [C1QQBP_MOUSE] & $0,000 \mathrm{EO}_{0}$ \\
\hline Q91VN6 & Probable ATP-dependent RNA helicase DDX41 OS=Mus musculus GN=Ddx41 PE=1 SV=2 - [DDX41_MOUSE] & $0,000 E 0$ \\
\hline Q62Q58 & La-related protein 1 OS=Mus musculus GN=Larp1 PE=1 SV=3 - [LARP1_MOUSE] & $0,000 E 0$ \\
\hline 088342 & WD repeat-containing protein $10 \mathrm{OS}=$ Mus musculus GN=Wdr1 PE=1 SV=3 - [WDR1_MOUSE] & $0,000 E 0$ \\
\hline Q9JKK1 & Syntaxin-6 OS=Mus musculus GN=Stx6 PE=1 SV=1 - [STX6_MOUSE] & $0,000 \mathrm{EO}$ \\
\hline P09405 & Nucleolin OS=Mus musculus GN=Ncl PE=1 SV=2 - [NUCL_MOUSE] & $0,000 E 0$ \\
\hline P32883-2 & Isoform 2B of GTPase KRas OS=Mus musculus GN=Kras - [RASK_MOUSE] & $0,000 E_{0}$ \\
\hline Q9W332 & Actin-related protein $2 / 3$ complex subunit $1 B$ OS=Mus musculus GN=Arpc1b PE=1SV=4 - [ARC1B_MOUSE] & $0,000 E 0$ \\
\hline
\end{tabular}

\begin{tabular}{|c|c|c|c|c|c|}
\hline OEO & $1,121 \mathrm{E} 6$ & 2,19448 & $4,647 ₹ 8$ & $7,238 E 8$ & Rpl10a \\
\hline $0,000 E 0$ & 0,000EO & 1,41088 & $2,356 \mathrm{E}_{8}$ & $6,112 E 8$ & Rpl15 \\
\hline, 00000 & $0,0,000=0$ & 6,61357 & 1,36488 & $1,946 E 8$ & Myo18a \\
\hline Doeo & 2,11455 & 2,150077 & $6,377 \mathrm{E}]$ & $7,6644 \mathrm{E} \mid$ & \\
\hline $0,000 E 0$ & $1,552 E 6$ & $\begin{array}{l}4,10688 \\
\end{array}$ & 8,72488 & $1,511 \mathrm{Eg}$ & Rps20 \\
\hline D00EO & 0,000E & $2,787 \in 7$ & 7,63117 & $9,605 E 7$ & Lrrfip1 \\
\hline $0000_{0}$ & 1,27966 & 2,10288 & $3,951 \mathrm{E}$ & 1,292299 & Rps15 \\
\hline .000E0 & \begin{tabular}{|l|l|l|} 
& $0,000 E$ \\
\end{tabular} & 1,45008 & $3,722 E 8$ & $2,636 E 88$ & Rpl24 \\
\hline $0,000 E=$ & 6,17866 & $1,554 \varepsilon_{8}$ & 1,96788 & 3,43998 & Rpl35a \\
\hline $0,000 E 0$ & \begin{tabular}{|c|}
$0,000 E O$ \\
\end{tabular} & 7,588566 & 1,559877 & 2,09667 & \\
\hline $0,000 E=$ & 3,05366 & $3,372 E 8$ & 4,57588 & 5,46558 & Myo6 \\
\hline 100000 & $\begin{array}{ll}3,780{ }^{2} \\
\end{array}$ & 8,81667 & 1,71668 & 3,689Е8 & Fxr1 \\
\hline $0,000 E 0$ & 0,000EO & 2,539977 & $3,693 \mathrm{E} 7$ & 5,11117 & Arf5 \\
\hline 0,00050 & 0,000EO & $2,693 E_{7}$ & $5,169 \mathrm{E}]$ & 6,08557 & Triobp \\
\hline $.000 E 0$ & $8,204 E 6$ & 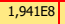 & 4,58988 & $1,303 E 9$ & Rps2 \\
\hline ,000E0 & $0,0,000=0$ & $1,527 \in]$ & 3,698877 & $7,532 E 7$ & Ralb \\
\hline $0,000 E 0$ & $1,205 E 7$ & 1,59778 & $3,718 E 8$ & $5,243 E 8$ & Hsp90 \\
\hline $0,000 E 0$ & 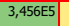 & 6,62557 & 1,48118 & $2,129 \pm 8$ & Rab1A \\
\hline ,000E0 & $0,000=0$ & 2,73867 & $3,928 \mathrm{E7}$ & $7,222 E 7$ & Fth1 \\
\hline $0,000 E 0$ & 1,19966 & 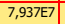 & 3,12588 & $2,375 E E$ & Tuba4a \\
\hline $0,000 E 0$ & \begin{tabular}{|c|}
$0,000 E 0$ \\
\end{tabular} & 1,43867 & $1,430 \mathrm{E} 7$ & 2,19067 & Arhgdib \\
\hline $0,000 E=0$ & $0,000=0$ & 8,19767 & $1,348 E 8$ & 2,98558 & Rps21 \\
\hline ,000E0 & ,, $000 E 0$ & 1,61118 & 2,253388 & 7,723388 & Rpl13a \\
\hline $0,000 E 0$ & $1,502 E 6$ & $1,097 E 8$ & $1,440 \times 8$ & $1,489 \mathrm{~EB}$ & Coro1c \\
\hline $0,000 E 0$ & ,, $000 E 0$ & 3,232E7 & $5,6977]$ & $\begin{array}{l}4,65667 \\
\end{array}$ & cct6a \\
\hline, 0000 & $7,429 E 5$ & $\begin{array}{l}4,48967] \\
\end{array}$ & $1,085 E 8$ & 1,93778 & Arhgef15 \\
\hline $0,000 E 0$ & $\begin{array}{l}, 000 E 0 \\
\end{array}$ & 2,649966 & 1,18007 & $1,535 \mathrm{E7}$ & \\
\hline $0,000 E 0$ & 0,000EO & 3,90447 & $8,581 \mathrm{E}$ & $1,947 \geq 8$ & Ckap4 \\
\hline $0,000 E 0$ & \begin{tabular}{|c|}
$0,000 E \mathrm{O}$ \\
\end{tabular} & 6,44067 & $1,816 \mathrm{E} 8$ & $2,818 E 8$ & Shank3 \\
\hline $0,000 E=0$ & 4,32966 & 2,256677 & $9,625 \mathrm{9} 7$ & 1,47008 & Tubb6 \\
\hline $0,000 E 0$ & 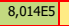 & $5,727 \in]$ & $1,472 \geq 8$ & $2,763 \mathrm{~EB}$ & Mybbp1a \\
\hline 0,000E0 & $4,676 \mathrm{E}$ & $3,787 \in 7$ & $9,668 \mathrm{E}]$ & 1,492288 & Pgk1 \\
\hline $0,000 E 0$ & \begin{tabular}{|l|l}
4,56115 \\
\end{tabular} & 1,19338 & $2,589 \pm 8$ & 2,63887 & Rpl21 \\
\hline $0,000 E=$ & $6,011 E 6$ & 7,841E7 & $8,065 \mathrm{E} 7$ & 6,165577 & Jup \\
\hline $0,000 E 0$ & $0,000=0$ & 1,26997 & 3,44667 & $5,2677 \mathrm{E}$ & Pgam5 \\
\hline $0,000 E 0$ & \begin{tabular}{|c|}
, $000 E 0$ \\
\end{tabular} & 1,51997 & 3,899E7] & $3,275 \mathrm{E7}$ & Sdpr \\
\hline ,000E & 0,000E0 & $1,26987]$ & 3,44667 & $5,267 \mathrm{E7}$ & Pgam5 \\
\hline ,000EO & \begin{tabular}{|l|l}
$7,835 E 55$ \\
\end{tabular} & 3,90967 & 7,19967 & $1,697 \pm 8$ & Rrbp1 \\
\hline $0,000 E=$ & $0,000=0$ & $5,57367]$ & $1,008 E 8$ & 1,99118 & Srsf1 \\
\hline $0,000 E 0$ & \begin{tabular}{|c|}
, $000 E 0$ \\
\end{tabular} & 1,86557 & 2,82337 & $\begin{array}{l}7,01677 \\
\end{array}$ & Uqcrq \\
\hline $0,000 E 0$ & $0,000=0$ & $\begin{array}{l}4,68446 \\
\end{array}$ & 2,488877 & 7,61777 & \\
\hline 0,000EO & 0,000EO & 6,329966 & $2,115 \mathrm{E}]$ & 3,23057 & Esd \\
\hline $0,000 E 0$ & $9,011 E 6$ & 2,35447 & $4,093 E 7$ & 7,549977 & Vcp \\
\hline 0,000EO & 0,000EO & $8,537 \mathrm{E}]$ & $1,164=8$ & $6,457 \mathrm{7} 7$ & Sars2 \\
\hline $0,000 E 0$ & 1,621166 & $1,657 € 7$ & 2,224477 & 3,30227 & Ldha \\
\hline $0,000 E 0$ & $0,000=0$ & $\begin{array}{l}4,323 E 6 \\
\end{array}$ & 1,32887 & 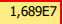 & Map6 \\
\hline $0,000 E 0$ & $2,209 E 6$ & 5,855E7 & $1,735 E 8$ & $3,073 E 8$ & Lima1 \\
\hline $0,000 E 0$ & \begin{tabular}{|l|}
5,484466 \\
\end{tabular} & $1,259 \mathrm{Eg}$ & $3,918 E 8$ & $3,976 \in 8$ & Tubb2a \\
\hline $0,000 E=0$ & 0,000E0 & $9,998 \mathrm{E}]$ & 2,08998 & $4,354 \mathrm{E}$ & Ptrf \\
\hline $0,000 E 0$ & $2,238 \mathrm{E}$ & 8,15177 & $\begin{array}{l}1,01988 \\
\end{array}$ & $1,458 E 8$ & Gnb2 \\
\hline $0,000 E 0$ & 0,000EO & $7,483 E 7$ & $1,794 \pm 8$ & $2,695 E 8$ & Akap2 \\
\hline $0,000 E 0$ & $3,110 E 6$ & $1,479 E 7$ & $4,467 \mathrm{ET}$ & 6,221177 & Aldoa \\
\hline $0,000 E=$ & $4,058 E 5$ & \begin{tabular}{|l|}
9,09977 \\
\end{tabular} & 1,70488 & 2,91998 & Svil \\
\hline $0,000 E 0$ & 0,000 0 & 3,86557 & $1,035 E 88$ & 1,00958 & lggap1 \\
\hline $0,000 E 0$ & 0,000EO & 2,18967 & $5,107 \in]$ & $\begin{array}{l}7,42667 \\
\end{array}$ & Cdk17 \\
\hline $0,000 E 0$ & $0,000=0$ & $2,757 \mathrm{E7}$ & $3,517 \mathrm{E}]$ & 7,06227 & Elavl1 \\
\hline $0,000 E 0$ & \begin{tabular}{ll|}
$9,589 \boxminus 55$ \\
\end{tabular} & 7,241E7 & 1,535588 & $3,570 \mathrm{~EB}$ & Myo10 \\
\hline $0,000 E 0$ & 0,000EO & 2,734477 & $5,632 E 7$ & 5,271177 & Twf1 \\
\hline $0,000 E 0$ & $1,208 \mathrm{EZ}$ & $1,533 \mathrm{Eg}$ & $2,676 \mathrm{E9}$ & $4,035 \mathrm{Eg}$ & Tpm2 \\
\hline 0,00050 & $0,000=0$ & 8,72257 & 1,468888 & 1,521188 & Ap $2 a 2$ \\
\hline $0,000 E 0$ & $0,000=0$ & 2,98007 & $1,009 \varepsilon 8$ & 1,23338 & Fscn1 \\
\hline 0,00000 & $0,000=0$ & $9,588 \mathrm{E}]$ & $1,233 E 8$ & $1,739 \mathrm{~EB}$ & Hip1 \\
\hline $0,000 E 0$ & $1,047 \boxminus 7$ & $1,214 \notin 8$ & 2,63228 & $5,693 E 8$ & Serbp1 \\
\hline $0,000 E 0$ & $0,000=0$ & $2,516 \mathrm{TE7}$ & 3,654477 & $4,793 E 7$ & Cep 131 \\
\hline $0,000 E 0$ & $0,000=0$ & 3,14667 & 6,150077 & $8,335 \mathrm{E}$ & Nedd4l \\
\hline $0,000 E 0$ & $2,753 E 6$ & $3,202 E 7$ & $7,252 \mathrm{~F}]$ & $8,884 \pm 7$ & Hnrnpm \\
\hline $0,000 E=0$ & $0,000=0$ & 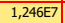 & 3,79387 & 2,48007 & Rhog \\
\hline $0,000 E 0$ & 0,0000 & $1,2922 \mathrm{E}$ & $3,729 E 7$ & $5,374 \pm 7$ & Sh3gl1 \\
\hline $0,000 E 0$ & $1,655 E 6$ & 2,36667 & 5,814877 & $7,797 \mathrm{E7}$ & Alyref \\
\hline $0,000 E 0$ & 0,000EO & $1,868 E 77$ & $2,397 \mathrm{E7}$ & 4,152277 & Gng12 \\
\hline $0,0000_{0}$ & $7,551 E 6$ & 2,49998 & $5,103=8$ & 1,052299 & Rp15 \\
\hline $0,000 E=0$ & 2,70855 & 1,61007 & 1,34867 & 3,11227 & Calu \\
\hline $0,000 E 0$ & $1,161 \mathrm{Y} 7$ & $4,6477 \mathrm{E}$ & 8,72357 & 9,79677 & SIC25a4 \\
\hline $0,000 E 0$ & \begin{tabular}{|c|}
$0,000 E \mathrm{O}$ \\
\end{tabular} & $2,845 E 8$ & 2,45998 & $2,062 E 8$ & Rpl31 \\
\hline $0,000 E=0$ & $3,828 E 5$ & 1,67008 & $3,233=8$ & 9,82228 & Rpl13 \\
\hline 0,00050 & $0,000=0$ & $1,357 \mathrm{E}$ & 7,17866 & 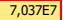 & Glipr2 \\
\hline $0,000 E=$ & $3,909 E 6$ & $1,340 E 88$ & $1,938 \mathrm{E} 8$ & $5,866 E 8$ & Rpl22|1 \\
\hline $0,000 E 0$ & 0,000EO & $3,742 E 7$ & 1,03448 & $1,349 E 8$ & Hnrnph2 \\
\hline $0,000 E 0$ & $0,000=0$ & $4,894 \pm 7$ & 8,61447 & 8,71567 & Add3 \\
\hline $0,000 E 0$ & 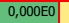 & $2,245 E 7$ & $\begin{array}{l}4,47997 \\
\end{array}$ & $3,445 E 7$ & cct4 \\
\hline $0,000 E$ & $0,000=0$ & 2,19667 & $1,144 E 8$ & 1,585588 & Lgals1 \\
\hline $0,000 E 0$ & 0,000E & $2,257 \mathrm{7}]$ & $1,515 E 7$ & $5,969 \mathrm{E7}$ & Eif2s1 \\
\hline 0,0006 & $6,534-6 \mid$ & 4,69997 & 1,00998 & $1,788 E 8$ & Fina \\
\hline 0,0006 & $0,000=0$ & 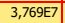 & $8,105 \mathrm{Z}$ & 1,16448 & Finc \\
\hline $0,000 E 0$ & $1,830 \mathrm{EZ}$ & 1,08117 & $8,322 \mathrm{E}$ & $1,538 E 8$ & C1qbp \\
\hline 0,000 & 0,000EO & $1,932 E 7$ & 3,78007 & $1,113 E 8$ & Ddx41 \\
\hline 0,0006 & , , 000E0 & 1,993E7 & 3,441E7 & $1,043 E 8$ & Larp1 \\
\hline 0,000 & $0,000=0$ & 3,82117 & 8,7667 & $1,533 \mathrm{E}$ & Wdr1 \\
\hline 0,000 & $0,0,000=0$ & 5,035566 & $5,487 \approx 6$ & 1,00007 & stx6 \\
\hline 0,000 & $1,477 \in 6$ & $9,349 \mathrm{EV}$ & 1,38258 & $4,549 \mathrm{~EB}$ & $\mathrm{NCl}$ \\
\hline 0,000 & $0,000=0$ & 5,6661166 & $2,507 \mathrm{~F}$ & 3,621177 & K \\
\hline, 00 & $0,0,000=0$ & 1,711188 & 3,299פ8 & $4,938 E 8$ & Arpc1t \\
\hline & $0,000=0$ & & & & \\
\hline
\end{tabular}




\begin{tabular}{|c|c|c|c|c|c|}
\hline P17751 & Triosephosphate isomerase OS=Mus musculus $\mathrm{GN}=\mathrm{Tpi1} \mathrm{PE}=1 \mathrm{SV}=4$ - [TPIS_MOUSE] & $0,0000_{0}$ & $0,000 \mathrm{E} O$ & $0,000 \mathrm{E} 0$ & 4,244E7 \\
\hline Q61029 & Lamina-associated polypeptide 2, isoforms beta/delta/epsilon/gamma OS=Mus musculus GN=Tmpo PE=1 SV=4 - [LAP2B_MOUSE] & $0,000 \mathrm{E} 0$ & $0,000 \mathrm{E} 0$ & $9,723 \mathrm{E} 5$ & $3,714 \mathrm{E} 7$ \\
\hline P08556 & GTPase NRas OS=Mus musculus GN=Nras PE=1 SV=1 - [RASN_MOUSE] & $0,000 E 0$ & $0,000 E 0$ & $0,000 E 0$ & $5,964 E 6$ \\
\hline Q6PCP5-2 & Isoform 2 of Mitochondrial fission factor OS=Mus musculus GN=Mff - [MFF_MOUSE] & $0,000 E 0$ & $0,000 \mathrm{E}_{0}$ & $0,000 \mathrm{E}^{2}$ & $1,152 \mathrm{E} 7$ \\
\hline Q61029-3 & Isoform Epsilon of Lamina-associated polypeptide 2, isoforms beta/delta/epsilon/gamma OS=Mus musculus GN=Tmpo - [LAP2B_MOUSE] & $0,0000_{0}$ & $0,000 \mathrm{E} 0$ & 9,723E5 & $3,714 \mathrm{E} 7$ \\
\hline P62071 & Ras-related protein R-Ras2 OS=Mus musculus GN=Rras2 PE=1 SV=1 - [RRAS2_MOUSE] & $0,000 E 0$ & $0,000 E 0$ & $0,000 \mathrm{E} 0$ & 7,704E7 \\
\hline Q6PCP5-3 & Isoform 3 of Mitochondrial fission factor OS=Mus musculus GN=Mff - [MFF_MOUSE] & $0,000 E 0$ & $0,000 \mathrm{EO}$ & $0,000 \mathrm{E} 0$ & $1,152 \mathrm{E} 7$ \\
\hline P50580-2 & Isoform 2 of Proliferation-associated protein $2 \mathrm{G} 4$ OS=Mus musculus GN=Pa2g4 - [PA2G4_MOUSE] & $0,000 E 0$ & $0,000 \mathrm{EO}$ & $0,000 E 0$ & $1,663 \mathrm{E} 7$ \\
\hline Q9QZD9 & Eukaryotic translation initiation factor 3 subunit I OS=Mus musculus GN=Eif3i PE=1 SV=1 - [EIF3I_MOUSE] & $0,000 \mathrm{E} 0$ & $0,000 \mathrm{E} 0$ & $0,000 \mathrm{E} 0$ & $3,220 \mathrm{E} 7$ \\
\hline P80314 & T-complex protein 1 subunit beta $\mathrm{OS}=$ Mus musculus GN=Cct2 PE=1 SV=4 - [TCPB_MOUSE] & $0,000 E 0$ & $0,000 \mathrm{EO}$ & $0,000 E 0$ & $2,422 E 7$ \\
\hline P48678 & Prelamin-A/C OS=Mus musculus GN=Lmna PE=1 SV=2 - [LMNA_MOUSE] & $0,000 E 0$ & $0,000 \mathrm{E} 0$ & $0,000 \mathrm{E} 0$ & $6,761 \mathrm{E} 6$ \\
\hline Q9WVC3 & Caveolin-2 OS=Mus musculus GN=Cav2 PE=1 SV=1- [CAV2_MOUSE] & $0,000 E 0$ & $0,000 E 0$ & $0,000 E 0$ & $1,301 E 7$ \\
\hline P62717 & 60S ribosomal protein L18a OS=Mus musculus GN=Rpl18a PE=1 SV=1 - [RL18A_MOUSE] & $0,000 E 0$ & $0,000 E 0$ & $0,000 E 0$ & $1,130 \mathrm{E} 8$ \\
\hline Q91v41 & Ras-related protein Rab-14 OS=Mus musculus GN=Rab14 PE=1 SV=3 - [RAB14_MOUSE] & $0,000 E 0$ & $0,000 \mathrm{EO}$ & 3,456E5 & $1,027 E 8$ \\
\hline Q61656 & Probable ATP-dependent RNA helicase DDX5 OS=Mus musculus GN=Ddx5 PE=1 SV=2 - [DDX5_MOUSE] & $0,000 E 0$ & $0,000 \mathrm{EO}$ & $0,000 \mathrm{EO}$ & $1,638 \mathrm{E} 8$ \\
\hline Q08509 & Epidermal growth factor receptor kinase substrate 8 OS=Mus musculus GN=Eps8 PE=1 SV=2 - [EPS8_MOUSE] & $0,000 E 0$ & $0,000 \mathrm{E}_{0}$ & $0,000 E 0$ & $5,863 E 7$ \\
\hline Q92105 & Chloride intracellular channel protein 1 OS=Mus musculus GN=Clic1 PE=1 SV=3 - [CLIC1_MOUSE] & $0,000 E 0$ & $0,000 \mathrm{E} 0$ & $0,000 \mathrm{E} 0$ & $7,145 \mathrm{E}$ \\
\hline Q9DBR7 & Protein phosphatase 1 regulatory subunit 12A OS=Mus musculus GN=Ppp1r12a PE $=1 \mathrm{SV}=2$ - [MYPT1_MOUSE] & $0,000 E 0$ & $0,000 \mathrm{E} 0$ & $0,000 \mathrm{E} 0$ & 7,218E7 \\
\hline Q9CYR0 & Single-stranded DNA-binding protein, mitochondrial OS=Mus musculus GN=SSbp1 PE=1 SV=1 - [SSBP_MOUSE] & $0,000 E 0$ & $0,000 \mathrm{EO}$ & 9,309E5 & $6,492 E 6$ \\
\hline Q9WUD1 & STIP1 homology and U box-containing protein 1 OS=Mus musculus GN=Stub1 PE=1 SV=1 - [CHIP_MOUSE] & $0,000 \mathrm{E} 0$ & $0,000 \mathrm{E} 0$ & $0,000 \mathrm{E}_{0}$ & $1,338 \mathrm{E} 7$ \\
\hline Q61194 & Phosphatidylinositol 4-phosphate 3-kinase C2 domain-containing subunit alpha OS=Mus musculus GN=Pik3c2a PE=1 SV=2 - [P3C2A_MOUSE] & $0,0000_{0}$ & $0,000 \mathrm{E} 0$ & $0,000 E_{0}$ & $1,325 E 8$ \\
\hline Q9WUK4 & Replication factor C subunit 2 OS=Mus musculus GN=RfC2 PE=1 SV=1 - [RFC2_MOUSE] & $0,0000_{0}$ & $0,000 \mathrm{EO}$ & $0,000 \mathrm{E} 0$ & $1,233 E 7$ \\
\hline P70460 & Vasodilator-stimulated phosphoprotein OS=Mus musculus GN=Vasp PE=1 $\mathrm{SV}=4$ - [VASP_MOUSE] & 0,000E0 & $0,000 \mathrm{E} 0$ & $0,000 \mathrm{E} 0$ & $2,338 \mathrm{E}$ \\
\hline P35980 & $60 S$ ribosomal protein $L 18$ OS=Mus musculus GN=Rp18 PE=1 SV=3 - [RL18_MOUSE] & $0,000 E 0$ & $0,000 E 0$ & 9,509E5 & $1,395 E 8$ \\
\hline Q6R891 & Neurabin-2 OS=Mus musculus GN=Ppp1rgb PE=1 SV=1 - [NEB2_MOUSE] & $0,000 E 0$ & $0,000 E 0$ & $0,0000_{0}$ & 2,384E7 \\
\hline Q8BGS2 & BolA-like protein 2 OS=Mus musculus GN=Bola2 PE=1 SV=1 - [BOLA2_MOUSE] & $0,000 E 0$ & $0,000 \mathrm{E} 0$ & $0,000 E 0$ & $8,013 \mathrm{E} 6$ \\
\hline Q61699-2 & Isoform HSP105-beta of Heat shock protein 105 kDa OS=Mus musculus GN=Hsph1 - [HS105_MOUSE] & $0,000 E 0$ & $0,000 \mathrm{E} 0$ & $0,000 E 0$ & $2,281 E 7$ \\
\hline P51150 & Ras-related protein Rab-7a OS=Mus musculus GN=Rab7a PE=1 SV=2 - [RAB7A_MOUSE] & $0,0000_{0}$ & $0,000 \mathrm{E} 0$ & $0,000 \mathrm{E} 0$ & $1,686 \mathrm{E} 7$ \\
\hline Q9D1C8 & Vacuolar protein sorting-associated protein 28 homolog OS=Mus musculus GN=Vps28 PE=1 SV=1 - [VPS28_MOUSE] & $0,000 E 0$ & $0,000 E 0$ & $0,000 E 0$ & 9,550E6 \\
\hline Q6AW69-5 & Isoform 5 of Cingulin-like protein 1 OS=Mus musculus GN=Cgnl1 - [CGNL1_MOUSE] & $0,000 E 0$ & $0,000 \mathrm{E} 0$ & $0,000 E 0$ & $1,233 E 7$ \\
\hline Pocos6 & Histone H2A.Z OS=Mus musculus GN=H2afz PE=1 SV=2 - [H2AZ_MOUSE] & $0,000 E 0$ & $0,000 E 0$ & $1,349 \mathrm{E}$ & 3,580E7 \\
\hline Q61598 & Rab GDP dissociation inhibitor beta OS=Mus musculus GN=Gdi2 PE=1 SV=1 - [GDIB_MOUSE] & $0,000 E 0$ & $0,000 E 0$ & $0,000 \mathrm{E} 0$ & $1,071 \mathrm{E}$ \\
\hline 008709 & Peroxiredoxin-6 OS=Mus musculus GN=Prdx6 PE=1 SV=3 - [PRDX6_MOUSE] & $0,000 E 0$ & $0,000 \mathrm{E} 0$ & $0,000 \mathrm{E} 0$ & $5,746 E 6$ \\
\hline Q92108-2 & Isoform L2 of Smoothelin OS=Mus musculus GN=Smtn - [SMTN_MOUSE] & $0,000 E 0$ & $0,000 \mathrm{EO}$ & $0,000 \mathrm{E} 0$ & 1,817 E7 \\
\hline P11440 & Cyclin-dependent kinase 1 OS=Mus musculus GN=Cdk1 PE=1 SV=3 - [CDK1_MOUSE] & $0,000 \mathrm{E}$ & $0,000 \mathrm{E} 0$ & $0,000 \mathrm{E}^{2}$ & \begin{tabular}{l|l}
$1,447 E 7$ \\
\end{tabular} \\
\hline P97461 & 40S ribosomal protein S5 OS=Mus musculus GN=Rps5 PE=1 SV=3 - [RS5_MOUSE] & $0,000 E 0$ & $0,000 \mathrm{EO}$ & $0,000 \mathrm{E} 0$ & $5,890 \mathrm{E}$ \\
\hline Q91WK2 & Eukaryotic translation initiation factor 3 subunit H OS=Mus musculus GN=Eif3h PE=1 SV=1 - [EIF3H_MOUSE] & $0,000 E 0$ & $0,000 \mathrm{E} 0$ & $0,000 \mathrm{E} 0$ & $1,755 \mathrm{E} 7$ \\
\hline P11031 & Activated RNA polymerase II transcriptional coactivator p15 OS=Mus musculus GN=Sub1 PE=1 SV=3 - [TCP4_MOUSE] & $0,000 E 0$ & $0,000 \mathrm{EO}$ & $7,455 E 5$ & $8,276 E 6$ \\
\hline 088746 & Target of Myb protein 1 OS=Mus musculus GN=Tom1 PE=1 SV=1 - [TOM1_MOUSE] & $0,000 E 0$ & $0,000 \mathrm{E} 0$ & $0,000 \mathrm{E} 0$ & 6,818E6 \\
\hline Q999P6 & Homer protein homolog $3 \mathrm{OS}=$ Mus musculus $\mathrm{GN}=$ Homer3 $\mathrm{PE}=1 \mathrm{SV}=2$ - [HOME3_MOUSE] & $0,0000_{0}$ & $0,000 E 0$ & $0,000 \mathrm{E} 0$ & $8,433 E 6$ \\
\hline P61979-3 & Isoform 3 of Heterogeneous nuclear ribonucleoprotein K OS=Mus musculus GN=Hnrnpk - [HNRPK_MOUSE] & $0,000 E 0$ & $0,000 E 0$ & $5,493 E 6$ & 7,494E7 \\
\hline 088952 & Protein lin-7 homolog C OS=Mus musculus GN=Lin7c PE=1 SV=2 - [LN7C_MOUSE] & $0,000 E 0$ & $0,000 \mathrm{E} 0$ & $0,000 \mathrm{E}_{0}$ & $1,219 \mathrm{E}$ \\
\hline P63094 & Guanine nucleotide-binding protein G(s) subunit alpha isoforms short OS=Mus musculus GN=Gnas PE=1 SV=1 - [GNAS2_MOUSE] & $0,000 E 0$ & $0,000 E 0$ & 9,807E4 & $4,359 \mathrm{E} 7$ \\
\hline 035737 & Heterogeneous nuclear ribonucleoprotein $\mathrm{H}$ OS=Mus musculus GN=Hnnph1 PE=1 SV=3 - [HNRH1_MOUSE] & $0,000 E 0$ & $0,000 \mathrm{E} 0$ & $0,000 \mathrm{E}_{0}$ & 6,18957 \\
\hline P46062 & Signal-induced proliferation-associated protein 1 OS=Mus musculus GN=Sipa1 PE=1 SV=2 - [SIPA1_MOUSE] & $0,000 E 0$ & $0,000 \mathrm{EO}$ & $0,000 \mathrm{E} 0$ & $9,330 \mathrm{EZ}$ \\
\hline Q03141-2 & Isoform 2 of MAP/microtubule affinity-regulating kinase 3 OS=Mus musculus GN=Mark3 - [MARK3_MOUSE] & $0,000 \mathrm{E} 0$ & $0,000 \mathrm{E} 0$ & $0,000 \mathrm{E} 0$ & 7,830E6 \\
\hline Q8BFR5 & Elongation factor Tu, mitochondrial OS=Mus musculus GN=Tufm PE=1 $1 \mathrm{VV}=1$ - [EFTU_MOUSE] & $0,000 E 0$ & $0,000 \mathrm{EO}$ & $0,000 \mathrm{E} 0$ & $1,445 E 7$ \\
\hline Q80WJ7 & Protein LYRIC OS=Mus musculus GN=Mtdh PE=1 SV=1 - [LYRIC_MOUSE] & $0,000 E 0$ & $0,000 \mathrm{E} 0$ & $0,000 E 0$ & $1,880 \mathrm{E} 7$ \\
\hline Q9CQH7 & Transcription factor BTF3 homolog 4 OS=Mus musculus GN=Btr314 PE=1 SV=1 - [BT3L4_MOUSE] & $0,000 E 0$ & $0,000 \mathrm{E} 0$ & $0,000 \mathrm{E}^{2}$ & $8,981 E 6$ \\
\hline Q9R0L6 & Pericentriolar material 1 protein OS=Mus musculus GN=Pcm1 1 PE=1 SV=2 - [PCM1_MOUSE] & $0,000 E 0$ & $0,000 \mathrm{EO}$ & $0,000 \mathrm{E} 0$ & $3,804 E 7$ \\
\hline Q9WTM5 & RuvB-like 2 OS=Mus musculus GN=Ruvb12 PE=1 SV=3 - [RUVB2_MOUSE] & $0,000 E 0$ & $0,000 E 0$ & 7,090E6 & $5,668 E 6$ \\
\hline Q9ESZ8-5 & Isoform 5 of General transcription factor II-I OS=Mus musculus GN=Gt22i - [GTF2I_MOUSE] & $0,000 E 0$ & $0,000 E 0$ & $0,000 E 0$ & 2,46957 \\
\hline Q8QZY1 & Eukaryotic translation initiation factor 3 subunit L OS=Mus musculus GN=Eif3l PE=1 SV=1 - [EIF3L_MOUSE] & $0,000 E 0$ & $0,000 E 0$ & $0,000 E_{0}$ & $1,678 E 7$ \\
\hline Q6ZWV7 & 60 ribosomal protein L35 OS=Mus musculus GN=Rpl35 PE=1 SV=1 - [RL35_MOUSE] & $0,000 E 0$ & $0,000 E_{0}$ & $0,000 E 0$ & $1,505 \mathrm{E} 8$ \\
\hline P23116 & Eukaryotic translation initiation factor 3 subunit A OS=Mus musculus GN=Eif3a PE=1 SV=5 - [EIF3A_MOUSE] & $0,000 \mathrm{E} 0$ & $0,000 \mathrm{E} 0$ & $0,000 E 0$ & $3,221 \mathrm{E}$ \\
\hline Q9JLQ0 & CD2-associated protein OS=Mus musculus GN=Cd2ap PE=1 SV=3 - [CD2AP_MOUSE] & $0,000 E 0$ & $0,000 \mathrm{E} 0$ & $0,000 \mathrm{E} 0$ & 9,052E6 \\
\hline Q922Y1 & UBX domain-containing protein 1 OS=Mus musculus GN=Ubxn1 PE=1SV=1 - [UBXN1_MOUSE] & $0,000 \mathrm{E} 0$ & $0,000 \mathrm{E} 0$ & $0,000 \mathrm{E} 0$ & $\begin{array}{ll}6,038 \mathrm{E} 6 \\
\end{array}$ \\
\hline Q8BL97-3 & Isoform 3 of Serine/arginine-rich splicing factor 7 OS=Mus musculus GN=Srsf7 - [SRSF7_MOUSE] & $0,000 E 0$ & $0,000 \mathrm{EO}$ & $0,000 E 0$ & $3,855 \mathrm{E}$ \\
\hline P84099 & 60 ribosomal protein L19 OS=Mus musculus GN=Rp19 PE=1 SV=1 - [RL19_MOUSE] & $0,000 E 0$ & $0,000 \mathrm{EO}$ & $0,000 \mathrm{E} 0$ & $1,113 \mathrm{E} 8$ \\
\hline P60335 & Poly(rC)-binding protein 1 OS=Mus musculus GN=Pcbp1 PE=1 SV=1- [PCBP1_MOUSE] & $0,000 E 0$ & $0,000 \mathrm{EO}$ & $6,208 E 5$ & $1,724 \mathrm{E} 7$ \\
\hline P46638 & Ras-related protein Rab-11B OS=Mus musculus GN=Rab11b PE=1 SV=3 - [RB11B_MOUSE] & $0,000 \mathrm{E} 0$ & $0,000 \mathrm{E}^{2}$ & $0,000 \mathrm{E} 0$ & $1,056 \mathrm{E} 7$ \\
\hline P58252 & Elongation factor $2 \mathrm{OS}=$ Mus musculus GN=Eef2 PE=1 $\mathrm{SV}=2$ - [EF2_MOUSE] & $0,000 E 0$ & $0,000 \mathrm{E} 0$ & $0,000 E 0$ & 4,746E7 \\
\hline Q6P1F6 & Serine/threonine-protein phosphatase 2A 55 kDa regulatory subunit B alpha isoform OS=Mus musculus GN=Ppp2r2a PE=1 SV=1 - [2ABA_MOUSE] & $0,000 E 0$ & $0,000 \mathrm{E} 0$ & $0,000 \mathrm{E} 0$ & $1,087 \mathrm{E}$ \\
\hline Q9QYC0 & Alpha-adducin OS=Mus musculus GN=Add1 PE=1 SV=2 - [ADDA_MOUSE] & $0,000 E 0$ & $0,000 \mathrm{EO}$ & $0,000 \mathrm{E}^{2}$ & $1,140 \mathrm{E} 8$ \\
\hline P62274 & $40 \mathrm{~S}$ ribosomal protein $\mathrm{S} 29$ OS=Mus musculus GN=Rps29 PE=3 SV=2 - [RS29_MOUSE] & $0,000 E 0$ & $0,000 \mathrm{E} 0$ & $0,000 E 0$ & $1,264 E 7$ \\
\hline Q8BU31 & Ras-related protein Rap-2c OS=Mus musculus GN=Rap2c PE=1 SV=1 - [RAP2C_MOUSE] & $0,000 E 0$ & $0,000 E 0$ & $0,000 E 0$ & $5,651 \mathrm{E} 6$ \\
\hline P63087-2 & Isoform Gamma-2 of Serine/threonine-protein phosphatase PP1-gamma catalytic subunit OS=Mus musculus GN=Ppp1cc - [PP1G_MOUSE] & $0,000 E_{0}$ & $0,000 \mathrm{E} 0$ & 2,353E6 & $5,878 E 7$ \\
\hline P84091 & AP-2 complex subunit mu OS=Mus musculus GN=Ap2m1 PE=1 SV=1 - [AP2M1_MOUSE] & $0,000 \mathrm{E} 0$ & $0,000 \mathrm{E} 0$ & $0,000 \mathrm{E} 0$ & 2,087E8 \\
\hline Q61187 & Tumor susceptibility gene 101 protein OS=Mus musculus GN=Tsg101 PE=1 SV=2 - [TS101_MOUSE] & $0,000 E 0$ & $0,000 \mathrm{E} 0$ & $0,000 E 0$ & $1,464 \mathrm{E}$ \\
\hline P42932 & T-complex protein 1 subunit theta OS=Mus musculus $\mathrm{GN}=\mathrm{CCt} 8 \mathrm{PE}=1 \mathrm{SV}=3$ - [TCPQ_MOUSE] & $0,000 E 0$ & $0,000 \mathrm{EO}$ & $6,390 \mathrm{E} 5$ & $2,318 \mathrm{E} 7$ \\
\hline P62849-2 & Isoform 2 of 40 S ribosomal protein S24 OS=Mus musculus GN=Rps24 - [RS24_MOUSE] & $0,000 E 0$ & $0,000 \mathrm{E} 0$ & $0,000 \mathrm{E} 0$ & $1,181 \mathrm{E} 8$ \\
\hline P97379-2 & Isoform B of Ras GTPase-activating protein-binding protein 2 OS=Mus musculus GN=G3bp2 - [G3BP2_MOUSE] & $0,000 E 0$ & $0,000 \mathrm{E} 0$ & 5,026E6 & \begin{tabular}{l|l}
$3,650 \mathrm{E} 7$ \\
\end{tabular} \\
\hline Q3TN34 & MICAL-like protein 2 OS=Mus musculus GN=Micall2 PE=1 SV=1 - [MILK2_MOUSE] & $0,000 E 0$ & $0,000 \mathrm{E} 0$ & $0,000 \mathrm{E} 0$ & $1,204 \mathrm{E} 7$ \\
\hline Q9DBC7 & CAMP-dependent protein kinase type I-alpha regulatory subunit OS=Mus musculus GN=Prkar1a PE=1 SV=3 - [KAP0_MOUSE] & $0,000 E 0$ & $0,000 \mathrm{E} 0$ & $0,000 E 0$ & $2,393 \mathrm{E}$ \\
\hline P67984 & 60 ribosomal protein L22 OS=Mus musculus GN=Rp122 PE=1 SV=2 - [RL22_MOUSE] & $0,000 E 0$ & $0,000 \mathrm{EO}$ & 2,391E6 & $1,809 \mathrm{E}$ \\
\hline Q8BG05 & Heterogeneous nuclear ribonucleoprotein $\mathrm{A} 3 \mathrm{OS}=$ Mus musculus GN=Hnrnpa3 PE=1 SV=1 - [ROA3_MOUSE] & $0,000 E 0$ & $0,000 E 0$ & $6,529 \mathrm{E}$ & $9,406 E 7$ \\
\hline Q6ZWY3 & 40S ribosomal protein S27-like OS=Mus musculus GN=Rps271 PE=1 SV=3 - [RS27L_MOUSE] & $0,000 E 0$ & $0,000 E_{0}$ & $1,094 \mathrm{E}$ & 2,132E8 \\
\hline Q6ZWU9 & 40 S ribosomal protein 527 OS=Mus musculus GN=Rps27 PE=1 $\mathrm{SV}=3-$ [RS27_MOUSE] & $0,000 E 0$ & $0,000 E_{0}$ & $1,094 \mathrm{E} 7$ & $2,519 E 8$ \\
\hline P05480 & Neuronal proto-oncogene tyrosine-protein kinase Src OS=Mus musculus GN=Src PE=1 SV=4 - [SRC_MOUSE] & $0,000 E 0$ & $0,000 \mathrm{E} 0$ & $2,114 \mathrm{E} 5$ & \begin{tabular}{ll|l}
$1,340 E 7$ \\
\end{tabular} \\
\hline Q8BG81 & Polymerase delta-interacting protein $30 \mathrm{OS}=$ Mus musculus GN=Poldip3 PE $=1 \mathrm{SV}=1$ - [PDIP3_MOUSE] & $0,000 E 0$ & $0,000 \mathrm{E} 0$ & $0,000 \mathrm{E} 0$ & $1,115 \mathrm{E}$ \\
\hline Q8BSD5 & SH2 domain-containing adapter protein $\mathrm{E}$ OS=Mus musculus $\mathrm{GN}=\mathrm{She} P \mathrm{PE}=1 \mathrm{SV}=1$ - [SHE_MOUSE] & $0,000 E 0$ & $0,000 E 0$ & $0,000 E 0$ & 1,152E7 \\
\hline Q01320 & DNA topoisomerase 2-alpha OS=Mus musculus GN=Top2a PE=1 SV=2 - [TOP2A_MOUSE] & $0,000 E 0$ & $0,000 E_{0}$ & $0,000 E 0$ & 2,88957 \\
\hline Q8CC35 & Synaptopodin OS=Mus musculus GN=Synpo PE=1 SV=2 - [SYNPO_MOUSE] & $0,000 \mathrm{EO}$ & $0,000 \mathrm{E} 0$ & $0,000 \mathrm{E} 0$ & $8,920 \mathrm{E} 6$ \\
\hline Q9CQE8 & UPF0568 protein C140rf166 homolog OS=Mus musculus PE=1 SV=1 - [CN166_MOUSE] & $0,000 E 0$ & $0,000 \mathrm{EO}$ & $0,000 \mathrm{E} 0$ & $1,244 \mathrm{EZ}$ \\
\hline Q8VHG2 & Angiomotin OS=Mus musculus GN=Amot PE=1 SV=3 - [AMOT_MOUSE] & $0,000 E 0$ & $0,000 E 0$ & $0,000 E 0$ & $1,728 E 7$ \\
\hline Q78PY7 & Staphylococcal nuclease domain-containing protein 1 OS=Mus musculus GN=Snd1 PE=1 SV=1 - [SND1_MOUSE] & $0,000 E 0$ & $0,000 \mathrm{EO}$ & $0,000 E 0$ & $2,632 E 7$ \\
\hline P62911 & $60 S$ ribosomal protein L32 OS=Mus musculus GN=Rpl32 PE=1 SV=2 - [RL32_MOUSE] & $0,000 E 0$ & $0,000 E 0$ & $0,000 \mathrm{E} 0$ & 2,833E7 \\
\hline P62855 & $40 \mathrm{~S}$ ribosomal protein $\mathrm{S26}$ OS=Mus musculus GN=Rps26 PE=1 SV=3 - [RS26_MOUSE] & $0,000 E 0$ & $0,000 \mathrm{E} 0$ & $0,000 E_{0}$ & $1,540 \mathrm{E}$ \\
\hline Q99k48 & Non-POU domain-containing octamer-binding protein OS=Mus musculus GN=Nono PE=1 SV=3 - [NONO_MOUSE] & $0,000 E 0$ & $0,000 \mathrm{E} 0$ & $0,000 E 0$ & $3,121 E 7$ \\
\hline Q92200 & Proteasome subunit alpha type-7 OS=Mus musculus GN=Psma7 PE=1 $1 \mathrm{SV}=1$ - [PSA7_MOUSE] & $0,000 E 0$ & $0,000 \mathrm{EO}$ & $0,000 \mathrm{E} 0$ & $5,100 E 6$ \\
\hline P27661 & Histone $\mathrm{H}_{2} \mathrm{AX}$ OS=Mus musculus GN=H2afx PE=1 SV=2 - [H2AX_MOUSE] & $1,458 E 7$ & $9,648 \mathrm{E} 6$ & $2,742 E 7$ & $5,055 \mathrm{E}$ \\
\hline P48036 & Annexin A5 OS=Mus musculus GN=Anxa5 PE=1 SV=1 - [ANXA5_MOUSE] & $0,000 E 0$ & $0,000 E_{0}$ & 3,591E6 & $8,024 E 6$ \\
\hline Q3V038 & Tetratricopeptide repeat protein 9A OS=Mus musculus $\mathrm{GN}=\mathrm{Ttc} 9 \mathrm{PE}=1 \mathrm{SV}=1$ - [TC9A_MOUSE] & $0,000 E 0$ & $0,000 E 0$ & $0,000 E 0$ & $8,639 E 6$ \\
\hline
\end{tabular}




\begin{tabular}{|c|c|c|c|c|c|}
\hline Q9QZQ1-2 & Isoform 1 of Afadin OS=Mus musculus GN=Afdn - [AFAD_MOUSE] & $0,000 \mathrm{EO}_{0}$ & $0,000 \mathrm{E} 0$ & $0,000 \mathrm{E} 0$ & $3,317 \mathrm{E}$ \\
\hline Q3UKC1 & Tax1-binding protein 1 homolog OS=Mus musculus GN=Tax1bp1 PE=1 SV=2 - [TAXB1_MOUSE] & $0,000 \mathrm{E} 0$ & $0,000 \mathrm{E} 0$ & $0,000 \mathrm{E} 0$ & $2,523 E 7$ \\
\hline Q8BHE0 & Proline-rich protein 11 OS=Mus musculus GN=Prr11 PE=2 SV=1 - [PRR11_MOUSE] & $0,000 E 0$ & $0,000 E 0$ & $0,000 \mathrm{E}_{0}$ & $1,001 E 7$ \\
\hline P63085 & Mitogen-activated protein kinase 1 OS=Mus musculus GN=Mapk1 PE=1 SV=3 - [MK01_MOUSE] & $0,000 E 0$ & $0,000 E 0$ & $0,000 \mathrm{E} 0$ & $1,495 E 7$ \\
\hline Q9EPU0-2 & Isoform 2 of Regulator of nonsense transcripts 1 OS=Mus musculus GN=Upf1 - [RENT1_MOUSE] & $0,000 \mathrm{EO}_{0}$ & $0,000 \mathrm{E} 0$ & $0,000 \mathrm{E} 0$ & $1,840 \mathrm{E} 7$ \\
\hline P26043 & Radixin OS=Mus musculus GN=Rdx PE=1 SV=3 - [RADI_MOUSE] & $0,000 E 0$ & $0,000 E 0$ & $2,157 E 5$ & $1,246 E 8$ \\
\hline Q99LE6 & ATP-binding cassette sub-family F member $2 \mathrm{OS}=$ Mus musculus $\mathrm{GN}=\mathrm{Abc2} 2 \mathrm{PE}=1 \mathrm{SV}=1$ - [ABCF2_MOUSE] & 0,000E0 & $0,000 E 0$ & $0,000 \mathrm{E} 0$ & 4,174E7 \\
\hline 070194 & Eukaryotic translation initiation factor 3 subunit D OS=Mus musculus GN=Eif3d PE=1 SV=2 - [EIF3D_MOUSE] & $0,000 E 0$ & $0,000 E 0$ & $0,000 \mathrm{E} 0$ & $1,504 \mathrm{E}$ \\
\hline P35278 & Ras-related protein Rab-5C OS=Mus musculus GN=Rab5c PE=1 SV=2 - [RABSC_MOUSE] & $0,000 E 0$ & $0,000 E 0$ & $0,000 \mathrm{E} 0$ & $4,074 E 6$ \\
\hline Q8R323 & Replication factor C subunit 3 OS=Mus musculus GN=RfC3 PE=1 SV=1 - [RFC3_MOUSE] & $0,000 E 0$ & $0,000 E 0$ & $2,006 E 6$ & $1,036 \mathrm{E} 7$ \\
\hline Q8BFW7 & Lipoma-preferred partner homolog OS=Mus musculus GN=Lpp PE=1 SV=1 - [LPP_MOUSE] & $0,000 E 0$ & $0,000 \mathrm{E} 0$ & $0,000 \mathrm{E}_{0}$ & $1,323 \mathrm{E} 7$ \\
\hline P07901 & Heat shock protein HSP 90-alpha OS=Mus musculus GN=Hsp90aa1 PE=1 SV=4 - [HS90A_MOUSE] & $1,255 \mathrm{E7}$ & $0,000 \mathrm{E} 0$ & 5,594E6 & $1,376 E 8$ \\
\hline Q69ZN7 & Myoferlin OS=Mus musculus GN=Myof PE=1 SV=2 - [MYOF_MOUSE] & $0,000 E 0$ & $0,000 \mathrm{E} 0$ & $0,000 \mathrm{E} 0$ & 2,787E7 \\
\hline P62317 & Small nuclear ribonucleoprotein Sm D2 OS=Mus musculus GN=Snrpd2 PE=1 SV=1 - [SMD2_MOUSE] & $0,000 E 0$ & $0,000 E 0$ & $0,000 \mathrm{E}_{0}$ & $2,805 E 6$ \\
\hline P32233 & Developmentally-regulated GTP-binding protein 1 OS=Mus musculus GN=Drg1 PE=1 SV=1 - [DRG1_MOUSE] & $0,000 E 0$ & $0,000 E 0$ & $0,000 \mathrm{E} 0$ & $6,982 E 6$ \\
\hline Q8R105 & Vacuolar protein sorting-associated protein $37 \mathrm{COS}=$ Mus musculus GN=Vps37c PE $=1$ SV=1 - [VP37C_MOUSE] & $0,000 \mathrm{EO}_{0}$ & $0,000 E 0$ & $0,000 \mathrm{E} 0$ & $1,138 \mathrm{E} 7$ \\
\hline P62331 & ADP-ribosylation factor 6 OS=Mus musculus GN=Arf6 PE=1 SV=2 - [ARF6_MOUSE] & 0,000E0 & $0,000 \mathrm{E} 0$ & $0,000 \mathrm{E} 0$ & $1,985 \mathrm{E} 7$ \\
\hline Q61235 & Beta-2-syntrophin OS=Mus musculus GN=Sntb2 PE=1 SV=2 - [SNTB2_MOUSE] & $0,000 E 0$ & $0,000 E 0$ & $0,000 \mathrm{E}^{-}$ & $1,425 \mathrm{E}$ \\
\hline Q999Y3 & GTPase IMAP family member 4 OS=Mus musculus GN=Gimap4 PE=1 SV=1 - [GIMA4_MOUSE] & $0,000 E 0$ & $0,000 E 0$ & $0,000 E 0$ & $1,139 \mathrm{EE}$ \\
\hline Q3UEB3-2 & Isoform 2 of Poly(U)-binding-splicing factor PUF60 OS=Mus musculus GN=Puf60 - [PUF60_MOUSE] & $0,000 E 0$ & $0,000 E 0$ & $0,000 \mathrm{E} 0$ & $1,578 \mathrm{E} 7$ \\
\hline Q61316 & Heat shock $70 \mathrm{kDa}$ protein 4 OS=Mus musculus GN=Hspa4 PE=1 SV=1 - [HSP74_MOUSE] & $0,000 E_{0}$ & $0,000 E 0$ & $0,000 \mathrm{E} 0$ & $1,679 \mathrm{EE}$ \\
\hline Q922Q4 & Pyrroline-5-carboxylate reductase 2 OS=Mus musculus GN=Pycr2 PE=1 SV=1 - [P5CR2_MOUSE] & $0,000 E 0$ & $0,000 E 0$ & $0,000 \mathrm{E} 0$ & $4,318 \mathrm{E} 7$ \\
\hline 070551 & SRSF protein kinase 1 OS=Mus musculus GN=Srpk1 PE=1 SV=2 - [SRPK1_MOUSE] & $0,000 E 0$ & $0,000 \mathrm{EO}$ & $0,000 E_{0}$ & $8,180 \mathrm{E} 6$ \\
\hline P35922-9 & Isoform 9 of Synaptic functional regulator FMR1 OS=Mus musculus GN=Fmr1 - [FMR1_MOUSE] & $0,000 E 0$ & $0,000 \mathrm{E} 0$ & $1,048 E 6$ & $6,566 \mathrm{E} 7$ \\
\hline P63001 & Ras-related C3 botulinum toxin substrate 1 OS=Mus musculus GN=Rac1 PE=1 SV=1 - [RAC1_MOUSE] & $0,000 E 0$ & $0,000 E 0$ & $0,0000_{0}$ & $3,428 \mathrm{E} 7$ \\
\hline P80316 & T-complex protein 1 subunit epsilon OS=Mus musculus GN=Cct5 PE=1 SV=1 - [TCPE_MOUSE] & $0,000 E 0$ & $0,000 E 0$ & $2,663 E 5$ & $6,466 \mathrm{E} 7$ \\
\hline P26040 & Ezrin OS=Mus musculus GN=Ezr PE=1 SV=3 - [EZRI_MOUSE] & $0,000 E 0$ & $0,000 E 0$ & $6,577 \pm 5$ & $1,246 E 8$ \\
\hline Q91VR2 & ATP synthase subunit gamma, mitochondrial OS=Mus musculus GN=Atp5c1 PE=1 SV=1 - [ATPG_MOUSE] & $0,0000_{0}$ & $0,000 \mathrm{E} 0$ & $0,000 \mathrm{E} 0$ & $1,589 \mathrm{E} 7$ \\
\hline Q60902 & Epidermal growth factor receptor substrate 15 -like 1 OS=Mus musculus GN=Eps1511 PE=1 SV=3 - [EP15R_MOUSE] & $0,000 E 0$ & $0,000 E 0$ & $0,000 \mathrm{E}_{0}$ & $1,358 \mathrm{E} 7$ \\
\hline Q05512-3 & Isoform 3 of Serine/threonine-protein kinase MARK2 OS=Mus musculus GN=Mark2 - [MARK2_MOUSE] & $0,000 E 0$ & $0,000 E 0$ & $0,000 \mathrm{E}_{0}$ & $1,491 E 7$ \\
\hline P60229 & Eukaryotic translation initiation factor 3 subunit E OS=Mus musculus GN=Eif3e PE=1 SV=1 - [EIF3E_MOUSE] & $0,000 E 0$ & $0,000 E 0$ & $0,000 \mathrm{E}_{0}$ & $1,019 \mathrm{E} 7$ \\
\hline P14069 & Protein S100-A6 OS=Mus musculus GN=S100a6 PE=1 1 SV=3 - [S10A6_MOUSE] & $0,000 \mathrm{E} 0$ & $0,000 E 0$ & $0,000 E 0$ & $1,257 \mathrm{E} 6$ \\
\hline P49817 & Caveolin-1 OS=Mus musculus GN=Cav1 PE=1 SV=1 - [CAV1_MOUSE] & $0,000 E 0$ & $0,000 E 0$ & $0,000 \mathrm{E} 0$ & $1,334 \mathrm{E} 8$ \\
\hline Q5SF07 & Insulin-like growth factor 2 mRNA-binding protein 2 OS=Mus musculus GN=Igf2bp2 PE=1 $\mathrm{SV}=1$ - [IF2B2_MOUSE] & $0,000 E 0$ & $0,000 E 0$ & $0,000 E_{0}$ & $2,192 E 7$ \\
\hline P17225 & Polypyrimidine tract-binding protein 1 OS=Mus musculus GN=Ptbp1 PE=1 SV=2 - [PTBP1_MOUSE] & $0,000 E 0$ & $0,000 E 0$ & $5,409 E 4$ & $7,832 E 7$ \\
\hline Q91VR5 & ATP-dependent RNA helicase DDX1 OS=Mus musculus GN=Ddx1 PE=1 SV=1 - [DDX1_MOUSE] & $0,000 E 0$ & $0,000 \mathrm{E} 0$ & $0,000 \mathrm{E} 0$ & $2,905 E 7$ \\
\hline Q60854 & Serpin B6 OS=Mus musculus GN=Serpinb6 PE=1 SV=1 - [SPB6_MOUSE] & $0,000 E 0$ & $0,000 E 0$ & $0,000 \mathrm{E} 0$ & $3,046 \mathrm{E} 7$ \\
\hline P57776 & Elongation factor 1-delta OS=Mus musculus GN=Eef1d PE=1 SV=3 - [EF1D_MOUSE] & $0,000 E 0$ & $0,000 E 0$ & $0,000 E 0$ & 7,344E7 \\
\hline Q99916 & Ras-related protein Rap-1b OS=Mus musculus GN=Rap1b PE=1SV=2 - [RAP1B_MOUSE] & $0,000 E 0$ & $0,000 E 0$ & $0,000 \mathrm{E} 0$ & $1,082 E 7$ \\
\hline P35279 & Ras-related protein Rab-6A OS=Mus musculus GN=Rab6a PE=1 SV=4 - [RAB6A_MOUSE] & $0,000 E_{0}$ & $0,000 E 0$ & $3,456 E 5$ & $3,992 \mathrm{E} 7$ \\
\hline Q9D4H4-2 & Isoform 2 of Angiomotin-like protein 1 OS=Mus musculus GN=Amot11 - [AMOL1_MOUSE] & $0,000 E 0$ & $0,000 E 0$ & $0,000 \mathrm{E}$ & 3,518E7 \\
\hline Q9CWK8 & Sorting nexin-2 OS=Mus musculus GN=Snx2 PE=1 SV=2 - [SNX2_MOUSE] & $0,000 \mathrm{E}_{0}$ & $0,000 \mathrm{E} 0$ & $2,776 \mathrm{E} 6$ & 1,623E6 \\
\hline P23242 & Gap junction alpha-1 protein OS=Mus musculus GN=Gja1 PE=1 SV=2 - [CXA1_MOUSE] & $0,000 E 0$ & $0,000 E 0$ & $0,000 \mathrm{E} 0$ & $7,979 E 7$ \\
\hline Q9D898 & Actin-related protein 2/3 complex subunit 5 -like protein OS=Mus musculus GN=Arpc5I PE=1 SV=1 - [ARP5L_MOUSE] & $0,000 E 0$ & $0,000 E 0$ & $0,000 \mathrm{E} 0$ & $2,921 \mathrm{E}$ \\
\hline P63321 & Ras-related protein Ral-A OS=Mus musculus GN=Rala PE=1 SV=1 - [RALA_MOUSE] & $0,000 E 0$ & $0,000 E 0$ & $0,000 E_{0}$ & $9,094 \mathrm{E} 6$ \\
\hline P11983 & T-complex protein 1 subunit alpha OS=Mus musculus GN=Tcp1 PE=1 SV=3 - [TCPA_MOUSE] & $0,000 \mathrm{E}$ & $0,000 \mathrm{E} 0$ & $2,616 \mathrm{E} 6$ & \begin{tabular}{l|l}
$3,239 \mathrm{E} 7$ \\
\end{tabular} \\
\hline Q60875-5 & Isoform 5 of Rho guanine nucleotide exchange factor 2 OS=Mus musculus GN=Arhgef2 - [ARHG2_MOUSE] & $0,000 E 0$ & $0,000 \mathrm{E} 0$ & $0,000 E 0$ & $1,999 \mathrm{E} 7$ \\
\hline Q99PT1 & Rho GDP-dissociation inhibitor 1 OS=Mus musculus GN=Arhgdia PE=1 SV=3 - [GDIR1_MOUSE] & $0,000 E 0$ & $0,000 E 0$ & $0,000 \mathrm{E}_{0}$ & $2,653 \mathrm{E} 7$ \\
\hline P61027 & Ras-related protein Rab-10 OS=Mus musculus GN=Rab10 PE=1 SV=1 - [RAB10_MOUSE] & $0,000 E 0$ & $0,000 E 0$ & $3,456 E 5$ & $5,919 \mathrm{E} 7$ \\
\hline Q9DCT8 & Cysteine-rich protein 2 OS=Mus musculus GN=Crip2 PE=1 SV=1 - [CRIP2_MOUSE] & $0,000 E 0$ & $0,000 E 0$ & $0,000 \mathrm{E} 0$ & $6,090 \mathrm{E} 7$ \\
\hline Q5SRX1 & TOM1-like protein 2 OS=Mus musculus GN=Tom12 PE=1 SV=1 - [TM1L2_MOUSE] & $0,000 E 0$ & $0,000 E 0$ & $0,000 \mathrm{E} 0$ & $6,885 E 6$ \\
\hline Q922X1-2 & Isoform 2 of Heterogeneous nuclear ribonucleoprotein F OS=Mus musculus GN=Hnrnpf - [HNRPF_MOUSE] & 0,000E0 & $0,000 E 0$ & $1,777 E 6$ & $5,494 \mathrm{E} 7$ \\
\hline E9Q5G3 & Kinesin-like protein KIF23 OS=Mus musculus GN=Kif23 PE=1 SV=1 - [KIF23_MOUSE] & $0,000 E 0$ & $0,000 E 0$ & $0,000 E 0$ & $1,194 \mathrm{E}$ \\
\hline 008529 & Calpain-2 catalytic subunit OS=Mus musculus GN=Capn2 PE=1 SV=4 - [CAN2_MOUSE] & $0,000 E 0$ & $0,000 E 0$ & $0,000 \mathrm{E} 0$ & $1,254 \mathrm{E} 7$ \\
\hline Q8C1B7-3 & Isoform 3 of Septin-11 OS=Mus musculus GN=Sept11 - [SEP11_MOUSE] & $0,000 E 0$ & $0,000 E 0$ & $4,406 E 6$ & $1,720 \mathrm{E} 7$ \\
\hline P42669 & Transcriptional activator protein Pur-alpha OS=Mus musculus GN=Pura PE=1 SV=1 - [PURA_MOUSE] & $0,000 E 0$ & $0,000 \mathrm{E} 0$ & $0,000 \mathrm{E}_{0}$ & $8,450 E 6$ \\
\hline P80313 & T-complex protein 1 subunit eta OS=Mus musculus GN=CCt7 PE=1 SV=1 - [TCPH_MOUSE] & 0,000E0 & $0,000 \mathrm{E} 0$ & $8,568 E 6$ & $2,270 \mathrm{E} 7$ \\
\hline P60122 & RuvB-like 1 OS=Mus musculus GN=Ruvbl1 PE=1 SV=1 - [RUVB1_MOUSE] & $0,000 E 0$ & $0,000 E 0$ & $0,000 \mathrm{E}_{0}$ & $6,639 E 6$ \\
\hline Q5F2E7 & Nuclear fragile X mental retardation-interacting protein 2 OS=Mus musculus GN=Nufip2 PE=1 SV=1 - [NUFP2_MOUSE] & $0,000 E 0$ & $0,000 E 0$ & $1,339 \mathrm{E} 6$ & $1,442 E 7$ \\
\hline Q90116 & 39 r ribosomal protein L14, mitochondrial OS=Mus musculus GN=Mrpl14 PE=1 SV=1 - [RM14_MOUSE] & $0,000 E 0$ & $0,000 E 0$ & $0,000 \mathrm{E} 0$ & $8,072 E 6$ \\
\hline P70698 & CTP synthase 1 OS=Mus musculus GN=Ctps1 PE=1 SV=2 - [PYRG1_MOUSE] & $0,000 \mathrm{E} 0$ & $0,000 \mathrm{E} 0$ & $0,000 \mathrm{E} 0$ & $1,140 \mathrm{E} 7$ \\
\hline Q8BK63-2 & Isoform 2 of Casein kinase I isoform alpha OS=Mus musculus GN=CSnk1a1 - [KC1A_MOUSE] & 0,000E0 & $0,000 E 0$ & $0,000 \mathrm{E} 0$ & $5,485 \mathrm{E} 6$ \\
\hline Q99445 & Eukaryotic translation initiation factor 2 subunit $2 \mathrm{OS}=$ Mus musculus $\mathrm{GN}=\mathrm{E}$ if2s2 PE $=1 \mathrm{SV}=1$ - [IF2B_MOUSE] & $0,000 E 0$ & $0,000 E 0$ & $0,000 E_{0}$ & $1,279 E 7$ \\
\hline P62315 & Small nuclear ribonucleoprotein Sm D1 OS=Mus musculus GN=Snrpd1 PE=1 SV=1 - [SMD1_MOUSE] & $0,000 E 0$ & $0,000 E 0$ & $2,089 E 6$ & $9,435 E 6$ \\
\hline P62192 & $26 \mathrm{~S}$ protease regulatory subunit $4 \mathrm{OS}=$ Mus musculus $\mathrm{GN}=\mathrm{Psmc1} \mathrm{PE}=1 \mathrm{SV}=1$ - [PRS4_MOUSE] & $0,000 E 0$ & $0,000 E 0$ & $0,000 E_{0}$ & $5,738 \mathrm{E} 6$ \\
\hline P17918 & Proliferating cell nuclear antigen OS=Mus musculus GN=Pcna PE=1 SV=2 - [PCNA_MOUSE] & $0,000 \mathrm{EO}$ & $0,000 \mathrm{E} 0$ & $8,188 E 5$ & $1,183 E 7$ \\
\hline Q3UQN2 & F-BAR domain only protein $2 \mathrm{OS}=$ Mus musculus $\mathrm{GN}=\mathrm{F}$ cho2 $\mathrm{PE}=1 \mathrm{SV}=1$ - [FCHO2_MOUSE] & $0,000 E 0$ & $0,000 \mathrm{E} 0$ & $0,000 \mathrm{E} 0$ & $3,931 \mathrm{E} 7$ \\
\hline Q99k51 & Plastin-3 OS=Mus musculus GN=Pls3 PE=1 SV=3 - [PLST_MOUSE] & $0,000 E 0$ & $0,000 E 0$ & $0,000 E_{0}$ & $8,897 \mathrm{E}$ \\
\hline Q9DB05 & Alpha-soluble NSF attachment protein OS=Mus musculus GN=Napa PE=1 SV=1 - [SNAA_MOUSE] & $0,000 E 0$ & $0,000 \mathrm{E} 0$ & $0,000 \mathrm{E}_{0}$ & $7,134 \mathrm{E} 6$ \\
\hline P57776-2 & Isoform 2 of Elongation factor 1-delta OS=Mus musculus GN=Eef1d - [EF1D_MOUSE] & $0,000 E 0$ & $0,000 E 0$ & $0,000 \mathrm{E} 0$ & $2,851 \mathrm{E} 7$ \\
\hline Q9DCH4 & Eukaryotic translation initiation factor 3 subunit F OS=Mus musculus GN=Eif3F PE=1 SV=2 - [EIF3F_MOUSE] & $0,000 E 0$ & $0,000 E 0$ & $0,000 \mathrm{E} 0$ & $2,204 E 7$ \\
\hline Q8C7U1 & NEDD4-binding protein 3 OS=Mus musculus GN=N4bp3 PE=1 SV=1 - [N4BP3_MOUSE] & $0,000 E 0$ & $0,000 E 0$ & $0,000 \mathrm{E}_{0}$ & $1,985 E 7$ \\
\hline P40124 & Adenylyl cyclase-associated protein 1 OS=Mus musculus GN=Cap1 PE=1 SV=4 - [CAP1_MOUSE] & $0,000 E 0$ & $0,000 E 0$ & $0,000 \mathrm{E}^{0}$ & $3,900 E 6$ \\
\hline P14115 & 60 r ribosomal protein L27a OS=Mus musculus GN=Rp127a PE=1 SV=5 - [RL27A_MOUSE] & $0,000 E 0$ & $0,000 \mathrm{E} 0$ & $3,543 E 5$ & $1,074 E 8$ \\
\hline Q6A065 & Centrosomal protein of $170 \mathrm{kDa}$ OS=Mus musculus GN=Cep170 PE=1 SV=2 - [CE170_MOUSE] & $0,000 E 0$ & $0,000 E 0$ & $2,926 E 5$ & 3,143E7 \\
\hline P25911-2 & Isoform 2 of Tyrosine-protein kinase Lyn OS=Mus musculus GN=Lyn - [LYN_MOUSE] & $0,000 E 0$ & $0,000 E 0$ & $0,000 \mathrm{E}_{0}$ & $1,185 E 7$ \\
\hline P35293 & Ras-related protein Rab-18 OS=Mus musculus GN=Rab18 PE=1 SV=2 - [RAB18_MOUSE] & $0,000 E 0$ & $0,000 E 0$ & $0,000 \mathrm{E} 0$ & $3,896 \mathrm{E} 6$ \\
\hline P26039 & Talin-1 OS=Mus musculus GN=Tln1 PE=1 SV=2 - [TLN1_MOUSE] & $0,000 E 0$ & $0,000 E 0$ & $0,000 \mathrm{E} 0$ & $1,185 \mathrm{E} 7$ \\
\hline Q9D6Z1 & Nucleolar protein 56 OS=Mus musculus GN=Nop56 PE=1 SV=2 - [NOP56_MOUSE] & $0,000 E 0$ & $0,000 E 0$ & $0,000 E_{0}$ & $5,334 E 6$ \\
\hline Q9ROP5 & Destrin OS=Mus musculus GN=Dstn PE=1 SV=3 - [DEST_MOUSE] & $0,000 E 0$ & $0,000 E 0$ & $0,000 \mathrm{E}^{-}$ & 7,401E6 \\
\hline Q922B2 & Aspartate--tRNA ligase, cytoplasmic OS=Mus musculus GN=Dars PE=1 SV=2 - [SYDC_MOUSE] & $0,000 E 0$ & $0,000 E 0$ & $0,000 \mathrm{E} 0$ & $8,969 \mathrm{E} 6$ \\
\hline Q7TMK9-2 & Isoform 2 of Heterogeneous nuclear ribonucleoprotein Q OS=Mus musculus GN=Syncrip - [HNRPQ_MOUSE] & $0,000 E 0$ & $0,000 E 0$ & $0,000 \mathrm{E} 0$ & 3,354E7 \\
\hline Q8R361 & Rab11 family-interacting protein 5 OS=Mus musculus GN=Rab11fip5 PE=1 $\mathrm{SV}=2$ - [RFIP5_MOUSE] & $0,000 E 0$ & $0,000 E 0$ & $0,000 \mathrm{E} 0$ & 1,404 E \\
\hline P46935 & E3 ubiquitin-protein ligase NEDD4 OS=Mus musculus GN=Nedd4 PE=1 SV=3 - [NEDD4_MOUSE] & $0,000 E 0$ & $0,000 \mathrm{E} 0$ & $0,000 \mathrm{E} 0$ & $1,561 \mathrm{E} 7$ \\
\hline Q61166 & Microtubule-associated protein RP/EB family member 1 OS=Mus musculus GN=Mapre1 PE=1 SV=3 - [MARE1_MOUSE] & $0,000 E_{0}$ & $0,000 E 0$ & $0,000 \mathrm{E}^{\circ}$ & $4,351 E 6$ \\
\hline Q8R411 & Myc target protein 1 OS=Mus musculus GN=Myct1 PE=1 SV=1 - [MYCT1_MOUSE] & $0,000 E 0$ & $0,000 \mathrm{E} 0$ & 1,124E6 & $1,514 E 8$ \\
\hline Q9QYJo & Dna] homolog subfamily A member 2 OS=Mus musculus GN=Dnaja2 PE=1 SV=1 - [DNJA2_MOUSE] & $0,000 E 0$ & $0,000 E 0$ & $0,000 \mathrm{E}_{0}$ & $2,477 E 7$ \\
\hline P35922-3 & Isoform 3 of Synaptic functional regulator FMR1 OS=Mus musculus GN=Fmr1 - [FMR1_MOUSE] & 0,000E0 & $0,000 \mathrm{E} 0$ & $1,048 E 6$ & $6,566 \mathrm{E} 7$ \\
\hline Q9D8NO & Elongation factor 1-gamma OS=Mus musculus GN=Eef1g PE=1 SV=3 - [EF1G_MOUSE] & $0,000 E 0$ & $0,000 \mathrm{E} 0$ & $1,369 \mathrm{E} 6$ & $5,285 E 7$ \\
\hline Q9DC51 & Guanine nucleotide-binding protein $\mathrm{G}(\mathrm{k})$ subunit alpha $\mathrm{OS}=$ Mus musculus $\mathrm{GN}=\mathrm{Gnai3} \mathrm{PE}=1 \mathrm{SV}=3$ - [GNAI3_MOUSE] & $0,000 E 0$ & $0,000 E 0$ & 7,794E4 & $3,831 E 7$ \\
\hline P49312 & Heterogeneous nuclear ribonucleoprotein A1 OS=Mus musculus $\mathrm{GN}=$ Hnrnpa1 PE=1 SV=2 - [ROA1_MOUSE] & $0,000 E 0$ & $0,000 \mathrm{E} 0$ & $2,262 E 6$ & $6,352 E 7$ \\
\hline Q91W92 & Cdc42 effector protein 1 OS=Mus musculus GN=Cdc42ep1 PE=1 SV=1 - [BORG5_MOUSE] & $0,000 E 0$ & $0,000 E 0$ & $0,000 \mathrm{E} 0$ & $1,837 \mathrm{E}$ \\
\hline P97379 & Ras GTPase-activating protein-binding & $0,000 E 0$ & $0,000 \mathrm{E} 0$ & $5,026 E 6$ & $3,650 \mathrm{E} 7$ \\
\hline
\end{tabular}




\begin{tabular}{|c|c|c|c|c|c|}
\hline P62874 & Guanine nucleotide-binding protein $\mathrm{G}(\mathrm{I} / \mathrm{G}(\mathrm{S}) / \mathrm{G}(\mathrm{T})$ subunit beta-1 $\mathrm{OS}=$ Mus musculus $\mathrm{GN}=\mathrm{Gnb1}$ PE=1 SV=3 - [GBB1_MOUSE] & $0,000 \mathrm{E} 0$ & $0,000 \mathrm{E} 0$ & $2,238 E 6$ & $8,151 \mathrm{E}$ \\
\hline Q9Q206 & Toll-interacting protein OS=Mus musculus GN=Tollip PE=1 SV=1 - [TOLIP_MOUSE] & $0,000 E 0$ & $0,000 \mathrm{E} 0$ & $0,000 \mathrm{E}_{0}$ & $8,920 \mathrm{E} 6$ \\
\hline Q50116 & Probable ATP-dependent RNA helicase DDX17 OS=Mus musculus GN=Dd×17 PE=1 SV=1 - [DDX17_MOUSE] & $0,000 E 0$ & $0,000 \mathrm{E} 0$ & $0,000 \mathrm{E}_{0}$ & 1,22788 \\
\hline P56873 & Sjoegren syndrome/scleroderma autoantigen 1 homolog OS=Mus musculus GN=SSsca1 PE=1 SV=1 - [SSA27_MOUSE] & $0,000 E 0$ & $0,000 E 0$ & $0,000 E 0$ & 4,624E6 \\
\hline Q91267 & SLIT-ROBO Rho GTPase-activating protein 2 OS=Mus musculus GN=Srgap2 PE=1 SV=2 - [SRGP2_MOUSE] & $0,000 E 0$ & $0,000 E 0$ & 3,66255 & $3,400 \mathrm{E} 7$ \\
\hline Q6PGB6-3 & Isoform 3 of $\mathrm{N}$-alpha-acetyltransferase $50 \mathrm{OS}=$ Mus musculus $\mathrm{GN}=\mathrm{Naa50}$ - [NAA50_MOUSE] & $0,000 \mathrm{E} 0$ & $0,000 E_{0}$ & $0,000 \mathrm{E} 0$ & $6,866 \mathrm{E} 5$ \\
\hline Q60865 & Caprin-1 OS=Mus musculus GN=Caprin1 PE=1 SV=2 - [CAPR1_MOUSE] & $0,000 E 0$ & $0,000 \mathrm{E}_{0}$ & $6,825 \mathrm{E} 6$ & $2,103 E 8$ \\
\hline Q8CHP5-2 & Isoform 2 of Partner of Y14 and mago OS=Mus musculus GN=Pym1 - [PYM1_MOUSE] & $0,000 \mathrm{E} 0$ & $0,000 \mathrm{E}_{0}$ & $1,961 \mathrm{E} 5$ & $2,149 \mathrm{E} 6$ \\
\hline Q9QX56-3 & Isoform E2 of Drebrin OS=Mus musculus GN=Dbn1 - [DREB_MOUSE] & $0,000 E 0$ & $0,000 \mathrm{E} 0$ & $0,000 \mathrm{E} 0$ & $5,168 \mathrm{E} 7$ \\
\hline Q8R1A4-2 & Isoform 2 of Dedicator of cytokinesis protein 7 OS=Mus musculus GN=Dock7 - [DOCK7_MOUSE] & $0,000 E 0$ & $0,000 \mathrm{EO}$ & $0,000 \mathrm{E} 0$ & $3,671 E 7$ \\
\hline P48024 & Eukaryotic translation initiation factor $1 \mathrm{OS}=$ Mus musculus $\mathrm{GN}=\mathrm{Eif1} 1 \mathrm{PE}=1 \mathrm{SV}=2$ - [EIF1_MOUSE] & $0,000 E 0$ & $0,000 E 0$ & $0,000 E 0$ & $3,642 E 5$ \\
\hline Q9D0B6 & Protein PBDC1 OS=Mus musculus GN=Pbdc1 PE=1 SV=1 - [PBDC1_MOUSE] & $0,000 E 0$ & $0,000 \mathrm{E} 0$ & $0,000 \mathrm{E} 0$ & $4,026 E 5$ \\
\hline P62754 & 40S ribosomal protein S6 OS=Mus musculus GN=Rps6 PE=1 SV=1 - [RS6_MOUSE] & $0,000 E 0$ & $0,000 \mathrm{E} 0$ & $2,070 \mathrm{E} 6$ & 6,530E7 \\
\hline 035295 & Transcriptional activator protein Pur-beta OS=Mus musculus GN=Purb PE=1 SV=3 - [PURB_MOUSE] & $0,000 E 0$ & $0,000 \mathrm{E} 0$ & $0,000 \mathrm{E} 0$ & $4,627 E 6$ \\
\hline P25911 & Tyrosine-protein kinase Lyn OS=Mus musculus GN=Lyn PE=1 SV=4- [LYN_MOUSE] & $0,000 E 0$ & $0,000 E 0$ & $0,000 \mathrm{E} 0$ & $1,185 E 7$ \\
\hline P58468 & Protein FAM207A OS=Mus musculus GN=Fam207a PE=1 SV=1 - [F207A_MOUSE] & $0,000 \mathrm{E} 0$ & $0,000 \mathrm{E} 0$ & $0,000 \mathrm{E} 0$ & 6,126E6 \\
\hline 008992 & Syntenin-1 OS=Mus musculus GN=Sdcbp PE=1 SV=1 - [SDCB1_MOUSE] & $0,000 \mathrm{E} 0$ & $0,000 \mathrm{E} 0$ & $0,000 \mathrm{E} 0$ & $4,975 \mathrm{E}$ \\
\hline Q8K1N2 & Pleckstrin homology-like domain family B member 2 OS=Mus musculus GN=Phldb2 PE=1 SV=2 - [PHLB2_MOUSE] & $0,000 E 0$ & $0,000 E 0$ & $0,000 E 0$ & $1,612 E 7$ \\
\hline P63330 & Serine/threonine-protein phosphatase 2A catalytic subunit alpha isoform OS=Mus musculus GN=Ppp2ca PE=1SV=1 - [PP2AA_MOUSE] & $0,000 E 0$ & $0,000 E 0$ & $0,000 E 0$ & $1,422 E 7$ \\
\hline P83882 & 60S ribosomal protein L36a OS=Mus musculus GN=Rp|36a PE=1 SV=2 - [RL36A_MOUSE] & $0,000 E 0$ & $0,000 E 0$ & $3,401 E 6$ & $1,254 \mathrm{E} 8$ \\
\hline $054824-2$ & Isoform 2 of Pro-interleukin-16 OS=Mus musculus GN=II16 - [IL16_MOUSE] & $0,000 E 0$ & $0,000 \mathrm{E} 0$ & $0,000 \mathrm{E} 0$ & $2,722 E 7$ \\
\hline $070325-2$ & Isoform Cytoplasmic of Phospholipid hydroperoxide glutathione peroxidase, mitochondrial OS=Mus musculus GN=Gpx4 - [GPX41_MOUSE] & $0,000 E 0$ & $0,000 E 0$ & $0,000 E 0$ & $5,742 E 6$ \\
\hline 089086 & RNA-binding protein 3 OS=Mus musculus GN=Rbm3 PE=1 SV=1 - [RBM3_MOUSE] & $0,000 \mathrm{E} 0$ & $0,000 E 0$ & $0,000 E 0$ & $2,435 \mathrm{E} 7$ \\
\hline Q8CC35-2 & Isoform 2 of Synaptopodin OS=Mus musculus GN=Synpo - [SYNPO_MOUSE] & $0,000 E 0$ & $0,000 \mathrm{E} 0$ & $0,000 E 0$ & $8,920 \mathrm{E} 6$ \\
\hline Q9CQ65 & S-methyl-5'-thioadenosine phosphorylase OS=Mus musculus GN=Mtap PE=1 SV=1 - [MTAP_MOUSE] & $0,000 E 0$ & $0,000 E_{0}$ & $0,000 \mathrm{E} 0$ & $1,504 E 7$ \\
\hline Q9DBY8 & Nuclear valosin-containing protein-like OS=Mus musculus GN=NvI PE=1 SV=1 - [NVL_MOUSE] & $0,000 E 0$ & $0,000 \mathrm{E} 0$ & $0,000 \mathrm{E} 0$ & 7,359E6 \\
\hline 054833 & Casein kinase II subunit alpha' OS=Mus musculus GN=CSnk2a2 PE=1 SV=1 - [CSK22_MOUSE] & $0,000 \mathrm{E} 0$ & $0,000 \mathrm{E} 0$ & $0,000 \mathrm{E} 0$ & 8,999E6 \\
\hline P55284 & Cadherin-5 OS=Mus musculus GN=Cdh5 PE=1 SV=2 - [CADH5_MOUSE] & $0,000 E 0$ & $0,000 E 0$ & $0,000 \mathrm{E} 0$ & $1,798 \mathrm{E} 8$ \\
\hline Q9QUR7 & Peptidyl-prolyl cis-trans isomerase NIMA-interacting 1 OS=Mus musculus GN=Pin1 PE=1 SV=1 - [PIN1_MOUSE] & $0,000 E 0$ & $0,000 \mathrm{E} 0$ & $0,000 E 0$ & $1,972 E 6$ \\
\hline Q9Z108 & Double-stranded RNA-binding protein Staufen homolog 1 OS=Mus musculus GN=Stau1 PE=1 SV=1- [STAU1_MOUSE] & $0,000 E 0$ & $0,000 E 0$ & $0,000 E 0$ & $1,767 \mathrm{E}$ \\
\hline P27546-3 & Isoform 3 of Microtubule-associated protein 4 OS=Mus musculus GN=Map4 - [MAP4_MOUSE] & $0,000 \mathrm{E}_{0}$ & $0,000 \mathrm{E} 0$ & $0,000 \mathrm{E} 0$ & 6,365E6 \\
\hline Q9JJA9 & General receptor for phosphoinositides 1-associated scaffold protein OS=Mus musculus GN=Grasp PE=1 $\mathrm{SV}=2$ - [GRASP_MOUSE] & $0,000 E 0$ & $0,000 E 0$ & $0,000 \mathrm{E} 0$ & $5,810 \mathrm{E} 6$ \\
\hline Q3UTJ2-3 & Isoform 3 of Sorbin and SH3 domain-containing protein 2 OS=Mus musculus GN=Sorbs2 - [SRBS2_MOUSE] & $0,000 \mathrm{E}_{0}$ & $0,000 \mathrm{E} 0$ & $0,000 \mathrm{E} 0$ & 3,852E6 \\
\hline Q9D0T1 & NHP2-like protein 1 OS=Mus musculus GN=Snu13 PE=1 SV=4 - [NH2L1_MOUSE] & $0,000 E 0$ & $0,000 E 0$ & $0,000 E 0$ & $3,638 \mathrm{E} 6$ \\
\hline P12399 & Protein CTLA-2-alpha OS=Mus musculus GN=Ctla2a PE=2 SV=2 - [CTL2A_MOUSE] & $0,000 E 0$ & $0,000 \mathrm{E} 0$ & $0,000 \mathrm{E} 0$ & $1,371 E 7$ \\
\hline Q9WTX6 & Cullin-1 OS=Mus musculus GN=Cul1 PE=1 SV=1 - [CUL1_MOUSE] & $0,000 E 0$ & $0,000 \mathrm{E} 0$ & $0,000 \mathrm{E}_{0}$ & $2,746 \mathrm{E} 7$ \\
\hline P62267 & 40S ribosomal protein S23 OS=Mus musculus GN=Rps23 PE=1 SV=3 - [RS23_MOUSE] & $0,000 E 0$ & $0,000 \mathrm{E} 0$ & $0,000 \mathrm{E} 0$ & $1,824 E 8$ \\
\hline Q921M7 & Protein FAM49B OS=Mus musculus GN=Fam49b PE=1 SV=1 - [FA49B_MOUSE] & $0,000 E 0$ & $0,000 E 0$ & $0,000 \mathrm{E} 0$ & $5,147 E 6$ \\
\hline Q8BFY7 & Protein FAM64A OS=Mus musculus GN=Fam64a PE=1 SV=1 - [FA64A_MOUSE] & $0,000 E 0$ & $0,000 E_{0}$ & $0,000 \mathrm{E}_{0}$ & $8,115 E 6$ \\
\hline Q9WUM3 & Coronin-1B OS=Mus musculus GN=Coro1b PE=1 SV=1 - [COR1B_MOUSE] & $0,000 E 0$ & $0,000 \mathrm{E} 0$ & $0,000 \mathrm{E} 0$ & 7,533E6 \\
\hline Q91296 & BMP-2-inducible protein kinase OS=Mus musculus GN=Bmp2k PE=1 SV=1 - [BMP2K_MOUSE] & $0,000 E 0$ & $0,000 \mathrm{E} 0$ & $0,000 \mathrm{E} 0$ & $4,933 \mathrm{E} 7$ \\
\hline Q8CGB3 & Uveal autoantigen with coiled-coil domains and ankyrin repeats $\mathrm{OS}=$ Mus musculus $\mathrm{GN}=$ Uaca PE $=1 \mathrm{SV}=2$ - [UACA_MOUSE] & $0,000 E 0$ & $0,000 E 0$ & $0,000 E 0$ & 3,024E7 \\
\hline Q9CQD1 & Ras-related protein Rab-5A OS=Mus musculus GN=Rab5a PE=1 SV=1 - [RAB5A_MOUSE] & $0,000 E 0$ & $0,000 \mathrm{E} 0$ & $0,000 \mathrm{E} 0$ & $1,978 E 6$ \\
\hline P62867 & 40S ribosomal protein S30 OS=Mus spicilegus GN=Fau PE=3 SV=1 - [RS30_MUSSI] & $0,000 E 0$ & $0,000 \mathrm{E} 0$ & $0,000 E 0$ & 3,120E7 \\
\hline P70313 & Nitric oxide synthase, endothelial OS=Mus musculus GN=Nos3 PE=1 SV=4 - [NOS3_MOUSE] & $0,000 E 0$ & $0,000 E 0$ & $0,000 E 0$ & $5,605 E 7$ \\
\hline Q6PHN9 & Ras-related protein Rab-35 OS=Mus musculus GN=Rab35 PE=1 SV=1 - [RAB35_MOUSE] & $0,000 \mathrm{E}^{2}$ & $0,000 \mathrm{E} 0$ & $3,456 \mathrm{E} 5$ & $6,493 \mathrm{E} 7$ \\
\hline Q8C863 & E3 ubiquitin-protein ligase Itchy OS=Mus musculus $\mathrm{GN}=$ Itch PE=1 SV=2 - [TTCH_MOUSE] & $0,000 E 0$ & $0,000 E 0$ & $0,000 E 0$ & $1,676 \mathrm{E} 7$ \\
\hline Q9JLB2 & MAGUK p55 subfamily member 5 OS=Mus musculus GN=Mpp5 PE=1 SV=1 - [MPP5_MOUSE] & $0,000 E 0$ & $0,000 \mathrm{E} 0$ & $0,000 E 0$ & $1,155 \mathrm{E} 7$ \\
\hline Q6PAM1 & Alpha-taxilin OS=Mus musculus GN=Txlna PE=1 SV=1 - [TXLNA_MOUSE] & $0,000 E_{0}$ & $0,000 \mathrm{E} 0$ & $0,000 \mathrm{E} 0$ & 1,945E7 \\
\hline Q99020 & Heterogeneous nuclear ribonucleoprotein A/B OS=Mus musculus $G N=H n r n p a b ~ P E=1 S V=1-\left[R O A A \_M O U S E\right]$ & $0,000 E 0$ & $0,000 \mathrm{E} 0$ & $1,457 \mathrm{E} 6$ & 1,603E7 \\
\hline Q92511 & ATPase family AAA domain-containing protein 3 OS=Mus musculus GN=Atad3 PE=1 SV=1 - [ATAD3_MOUSE] & $0,000 \mathrm{E} 0$ & $0,000 \mathrm{E}_{0}$ & $0,000 \mathrm{E} 0$ & $1,707 E 7$ \\
\hline P15864 & Histone H1.2 OS=Mus musculus $\mathrm{GN}=$ Hist1h1c PE=1 SV=2 - [H12_MOUSE] & $0,000 E 0$ & $0,000 \mathrm{E}_{0}$ & $6,982 E 5$ & 6,443E7 \\
\hline Q80X85 & $28 \mathrm{~S}$ ribosomal protein S7, mitochondrial OS=Mus musculus GN=Mrps7 PE=1 SV=1 - [RT07_MOUSE] & $0,000 E 0$ & $0,000 E 0$ & $0,000 E 0$ & $2,532 E 6$ \\
\hline Q9D824-4 & Isoform 4 of Pre-mRNA 3'-end-processing factor FIP1 OS=Mus musculus GN=Fip111 - [FIP1_MOUSE] & $0,000 E 0$ & $0,000 \mathrm{E} 0$ & $0,000 \mathrm{E} 0$ & $5,832 \mathrm{E} 6$ \\
\hline Q3UZ39-2 & Isoform 2 of Leucine-rich repeat flightless-interacting protein 1 OS=Mus musculus GN=Lrrfip1 - [LRRF1_MOUSE] & $0,000 E 0$ & $0,000 \mathrm{E} 0$ & $0,000 \mathrm{E}_{0}$ & $4,588 \mathrm{E} 7$ \\
\hline 035643 & AP-1 complex subunit beta-1 OS=Mus musculus GN=Ap1b1 PE=1 SV=2 - [AP1B1_MOUSE] & $0,000 E 0$ & $0,000 E 0$ & $0,000 E 0$ & $2,272 E 8$ \\
\hline Q9CSN1 & SNW domain-containing protein $1 \mathrm{OS}=$ Mus musculus GN=SnW1 PE=1 SV=3 - [SNW1_MOUSE] & 0,000E0 & $0,000 \mathrm{E} 0$ & $0,000 \mathrm{E} 0$ & $3,256 \mathrm{E} 6$ \\
\hline Q8C111 & Guanine nucleotide-binding protein-like 3 OS=Mus musculus GN=Gnl3 PE=1 SV=2 - [GNL__MOUSE] & $0,000 E 0$ & $0,000 E 0$ & $0,000 E 0$ & $1,329 \mathrm{E} 7$ \\
\hline Q8VEK3 & Heterogeneous nuclear ribonucleoprotein U OS=Mus musculus GN=Hnrnpu PE $=1 \mathrm{SV}=1$ - [HNRPU_MOUSE] & $0,000 E 0$ & $0,000 E 0$ & $0,000 \mathrm{E} 0$ & $5,206 \mathrm{E} 7$ \\
\hline P34022 & Ran-specific GTPase-activating protein OS=Mus musculus GN=Ranbp1 PE=1 SV=2 - [RANG_MOUSE] & $0,000 E 0$ & $0,000 E 0$ & $0,000 \mathrm{E} 0$ & $5,821 E 6$ \\
\hline Q8CBW3-5 & Isoform 5 of Abl interactor 1 OS=Mus musculus GN=Abi1 - [ABI1_MOUSE] & $0,000 \mathrm{E} 0$ & $0,000 \mathrm{E} 0$ & $0,000 \mathrm{E} 0$ & $1,595 \mathrm{E} 7$ \\
\hline Q92315 & U4/U6.U5 tri-SnRNP-associated protein 1 OS=Mus musculus GN=Sart1 PE=1 SV=1 - [SNUT1_MOUSE] & $0,000 E 0$ & $0,000 E_{0}$ & $0,000 \mathrm{E}_{0}$ & $4,632 \mathrm{E} 6$ \\
\hline Q3UJ89-2 & Isoform 2 of Enhancer of mRNA-decapping protein 4 OS=Mus musculus GN=Edc4 - [EDC4_MOUSE] & $0,000 E 0$ & $0,000 E 0$ & $0,000 E 0$ & $1,246 \mathrm{E} 7$ \\
\hline Q60631 & Growth factor receptor-bound protein $2 \mathrm{OS}=$ Mus musculus GN=Grb2 PE=1 SV=1 - [GRB2_MOUSE] & $0,000 E 0$ & $0,000 E 0$ & $0,000 E 0$ & $6,084 E 6$ \\
\hline Q91VH2 & Sorting nexin-9 OS=Mus musculus GN=Snx9 PE=1 SV=1 - [SNX9_MOUSE] & $0,000 E 0$ & $0,000 \mathrm{E} 0$ & $0,000 \mathrm{E} 0$ & $4,403 E 7$ \\
\hline Q9QY13 & Dna] homolog subfamily C member 7 OS=Mus musculus GN=Dnajc7 PE=1 SV=2 - [DNJC7_MOUSE] & $0,000 E 0$ & $0,000 \mathrm{E} 0$ & $0,000 \mathrm{E} 0$ & 3,884E6 \\
\hline Q9JM76 & Actin-related protein $2 / 3$ complex subunit 3 OS=Mus musculus GN=Arpc3 PE=1 SV=3 - [ARPC3_MOUSE] & $0,000 E 0$ & $0,000 E 0$ & 7,38055 & 9,633E7 \\
\hline Q8VDN2 & Sodium/potassium-transporting ATPase subunit alpha-1 OS=Mus musculus GN=Atp1a1 PE=1 SV=1 - [AT1A1_MOUSE] & $0,000 E 0$ & $0,000 \mathrm{E} 0$ & $0,000 \mathrm{E} 0$ & $1,542 E 7$ \\
\hline Q5SV80 & Unconventional myosin-XIX OS=Mus musculus GN=Myo19 PE=2 SV=1 - [MYO19_MOUSE] & $0,000 E 0$ & $0,000 E 0$ & $0,000 E 0$ & $1,546 \mathrm{E} 7$ \\
\hline Q8BP92 & Reticulocalbin-2 OS=Mus musculus GN=Rcn2 PE=1 SV=1 - [RCN2_MOUSE] & $0,000 \mathrm{E} 0$ & $0,000 \mathrm{E}_{0}$ & $0,000 \mathrm{E} 0$ & $6,868 \mathrm{E} 6$ \\
\hline Q3UPF5 & Zinc finger CCCH-type antiviral protein 1 OS=Mus musculus GN=ZC3hav1 PE=1 SV=1 - [ZCCHV_MOUSE] & $0,000 E 0$ & $0,000 \mathrm{E} 0$ & $0,000 \mathrm{E} 0$ & $1,162 E 7$ \\
\hline Q907X3 & Dual specificity protein phosphatase 3 OS=Mus musculus GN=Dusp3 PE=1 SV=1 - [DUS3_MOUSE] & $0,000 \mathrm{E} 0$ & $0,000 \mathrm{E} 0$ & $0,000 \mathrm{E} 0$ & $5,026 \mathrm{E} 6$ \\
\hline Q8BGD9 & Eukaryotic translation initiation factor $4 \mathrm{~B}$ OS=Mus musculus $\mathrm{GN}=\mathrm{Eif} 4 \mathrm{~b} P \mathrm{PE}=1 \mathrm{SV}=1$ - [IF4B_MOUSE] & $0,000 E 0$ & $0,000 E 0$ & $0,000 \mathrm{E} 0$ & $2,896 \mathrm{E} 7$ \\
\hline P19324 & Serpin H1 OS=Mus musculus GN=Serpinh1 PE=1 SV=3 - [SERPH_MOUSE] & $0,000 E 0$ & $0,000 \mathrm{E} 0$ & $0,000 E 0$ & $1,283 E 7$ \\
\hline Q993X4 & Eukaryotic translation initiation factor 3 subunit M OS=Mus musculus GN=EFf3m PE=1 SV=1 - [EIF3M_MOUSE] & $0,000 E 0$ & $0,000 E 0$ & $0,000 E 0$ & $2,294 E 6$ \\
\hline Q99k43 & Protein regulator of cytokinesis 1 OS=Mus musculus GN=Prc1 PE=1 SV=2 - [PRC1_MOUSE] & $0,000 E 0$ & $0,000 E 0$ & $0,000 E 0$ & 9,965E6 \\
\hline Q61510 & E3 ubiquitin/ISG15 ligase TRIM25 OS=Mus musculus GN=Trim25 PE=1 SV=2 - [TRI25_MOUSE] & $0,000 \mathrm{EO}$ & $0,000 \mathrm{E} 0$ & $0,000 \mathrm{E} 0$ & 3,692E6 \\
\hline P27773 & Protein disulfide-isomerase A3 OS=Mus musculus GN=Pdia3 PE=1 SV=2 - [PDIA3_MOUSE] & $0,000 \mathrm{E} 0$ & $0,000 \mathrm{E} 0$ & $0,000 E 0$ & 7,711E6 \\
\hline Q9JMBO & G kinase-anchoring protein 1 OS=Mus musculus GN=Gkap1 PE=1 SV=1 - [GKAP1_MOUSE] & $0,000 E 0$ & $0,000 E 0$ & $0,000 E 0$ & $4,392 E 6$ \\
\hline Q9CQW9 & Interferon-induced transmembrane protein $3 \mathrm{OS}=$ Mus musculus $\mathrm{GN}=$ =Iftm3 PE=1 $\mathrm{SV}=1$ - [IFM3_MOUSE] & $0,000 E 0$ & $0,000 E 0$ & $0,000 E 0$ & $5,875 \mathrm{E} 7$ \\
\hline P49710 & Hematopoietic lineage cell-specific protein OS=Mus musculus GN=Hcls1 PE=1 SV=2 - [HCLS1_MOUSE] & $0,000 E 0$ & $0,000 \mathrm{E} 0$ & $0,000 \mathrm{E}_{0}$ & $5,149 \mathrm{E} 6$ \\
\hline P63024 & Vesicle-associated membrane protein 3 OS=Mus musculus GN=Vamp3 PE=1 SV=1 - [VAMP3_MOUSE] & $0,000 E 0$ & $0,000 \mathrm{E} 0$ & $0,000 \mathrm{E} 0$ & $8,104 E 6$ \\
\hline Q61990-2 & Isoform 2 of Poly(rC)-binding protein 2 OS=Mus musculus GN=Pcbp2 - [PCBP2_MOUSE] & $0,000 E 0$ & $0,000 E 0$ & $0,000 \mathrm{E} 0$ & $2,185 E 7$ \\
\hline Q99KN9 & Clathrin interactor 1 OS=Mus musculus GN=Clint1 PE=1 SV=2 - [EPN4_MOUSE] & $0,000 E 0$ & $0,000 \mathrm{E} 0$ & $0,000 \mathrm{E}_{0}$ & $5,761 \mathrm{E} 7$ \\
\hline Q9W095 & Pleckstrin homology-like domain family A member 3 OS=Mus musculus GN=Phlda3 PE=1 SV=1 - [PHLA3_MOUSE] & $0,000 E 0$ & $0,000 \mathrm{E} 0$ & $0,000 \mathrm{E} 0$ & $8,774 E 6$ \\
\hline P98083-2 & Isoform p52Shc of SHC-transforming protein 1 OS=Mus musculus GN=Shc1 - [SHC1_MOUSE] & $0,000 E 0$ & $0,000 \mathrm{E} 0$ & $0,000 \mathrm{E} 0$ & $2,970 \mathrm{E} 5$ \\
\hline Q6A0D4 & Raftlin OS=Mus musculus GN=Rftn1 PE=1 SV=4 - [RFTN1_MOUSE] & $0,000 E 0$ & $0,000 E 0$ & 2,147E5 & 3,394E6 \\
\hline P24369 & Peptidyl-prolyl cis-trans isomerase B OS=Mus musculus GN=Ppib PE=1 SV=2 - [PPIB_MOUSE] & $0,000 E 0$ & $0,000 \mathrm{E} 0$ & $0,000 \mathrm{E} 0$ & $2,723 \mathrm{E} 6$ \\
\hline Q7TMB8 & Cytoplasmic FMR1-interacting protein 1 OS=Mus musculus GN=Cyfip1 PE=1 SV=1- [CYFP1_MOUSE] & $0,000 E 0$ & $0,000 \mathrm{E} 0$ & $1,056 \mathrm{E} 6$ & $3,183 \mathrm{E} 7$ \\
\hline Q8CGB6-2 & Isoform 2 of Tensin-2 OS=Mus musculus GN=Tns2 - [TNS2_MOUSE] & $0,000 E 0$ & $0,000 E 0$ & $0,000 \mathrm{E} 0$ & $1,830 \mathrm{E} 7$ \\
\hline Q80UG5-3 & Isoform 3 of Septin-9 OS=Mus musculus GN=Sept9 - [SEPT9_MOUSE] & $0,000 E 0$ & $0,000 \mathrm{E} 0$ & $0,000 E 0$ & $9,735 E 6$ \\
\hline Q8BIE6 & FERM domain-containing protein 4A OS=Mus musculus GN=Frmd4a PE=1SV=2 - [FRM4A_MOUSE] & $0,000 E 0$ & $0,000 \mathrm{E} 0$ & $0,000 \mathrm{E}_{0}$ & $1,217 \mathrm{E}$ \\
\hline P21279 & Guanine nucleotide-binding protein $\mathrm{G}(q)$ subunit alpha $\mathrm{OS}=$ Mus musculus $\mathrm{GN}=\mathrm{Gnaq} P \mathrm{PE}=1 \mathrm{SV}=4$ - [G & $0,000 E 0$ & $0,000 \mathrm{E} 0$ & $0,000 \mathrm{E} 0$ & $7,952 E 6$ \\
\hline
\end{tabular}




\begin{tabular}{|c|c|c|c|c|c|}
\hline Q9CR26 & Vacuolar protein sorting-associated protein VTA1 homolog OS=Mus musculus GN=Vta1 PE=1 SV=1 - [VTA1_MOUSE] & $0,000 \mathrm{E} 0$ & $0,000 \mathrm{E} 0$ & $0,000 \mathrm{E} 0$ & $7,016 \mathrm{E} 6$ \\
\hline Q8CGC4 & Protein LSM14 homolog B OS=Mus musculus GN=LSm14b PE=1 SV=3 - [LS14B_MOUSE] & $0,000 E 0$ & $0,000 E 0$ & $0,000 \mathrm{EO}$ & $5,806 \mathrm{EE}$ \\
\hline 009131 & Glutathione S-transferase omega-1 OS=Mus musculus GN=Gsto1 PE=1 SV=2 - [GSTO1_MOUSE] & $0,000 E 0$ & $0,000 E 0$ & $0,000 \mathrm{E} 0$ & $2,292 E 6$ \\
\hline Q8C8U0-3 & Isoform 3 of Liprin-beta-1 OS=Mus musculus GN=Ppfibp1 - [LPB1_MOUSE] & $0,0000_{0}$ & $0,000 \mathrm{E} 0$ & $0,0000_{0}$ & 6,686E6 \\
\hline Q9ZON1 & Eukaryotic translation initiation factor 2 subunit 3, $X$-linked OS=Mus musculus GN=EFif2s3x PE=1 SV=2 - [IF2G_MOUSE] & $0,000 \mathrm{E} 0$ & $0,000 \mathrm{E} 0$ & $0,000 \mathrm{E} 0$ & $2,187 \mathrm{E}$ \\
\hline Q3TWW8 & Serine/arginine-rich splicing factor 6 OS=Mus musculus GN=Srsf6 PE=1 SV=1 - [SRSF6_MOUSE] & $0,000 \mathrm{E} 0$ & $0,000 \mathrm{E} 0$ & $0,000 \mathrm{E} 0$ & 6,749E7 \\
\hline Q9DC28-2 & Isoform 2 of Casein kinase I isoform delta OS=Mus musculus GN=CSnk1d - [KC1D_MOUSE] & $0,000 \mathrm{E} 0$ & $0,000 \mathrm{E} 0$ & $0,000 \mathrm{E} 0$ & $1,802 E 7$ \\
\hline Q9Z1Z2 & Serine-threonine kinase receptor-associated protein OS=Mus musculus GN=Strap PE=1 SV=2 - [STRAP_MOUSE] & $0,000 \mathrm{E} 0$ & $0,000 \mathrm{E} 0$ & $0,000 \mathrm{E} 0$ & $2,811 \mathrm{E} 6$ \\
\hline Q9D019 & Arginine--tRNA ligase, cytoplasmic OS=Mus musculus GN=Rars PE=1 SV=2 - [SYRC_MOUSE] & $0,000 \mathrm{E} 0$ & $0,000 E 0$ & $0,000 \mathrm{E} 0$ & $1,698 \mathrm{E} 7$ \\
\hline Q7TSC1 & Protein PRRC2A OS=Mus musculus GN=Prrc2a PE=1 SV=1 - [PRC2A_MOUSE] & $0,000 \mathrm{E} 0$ & $0,000 \mathrm{E} 0$ & $0,000 \mathrm{E} 0$ & $1,548 \mathrm{E} 7$ \\
\hline Q9Z1X4-2 & Isoform 2 of Interleukin enhancer-binding factor $3 \mathrm{OS}=$ Mus musculus GN=Iff3 - [LFF_MOUSE] & $0,000 \mathrm{E} 0$ & $0,000 E 0$ & $0,000 E_{0}$ & $1,067 \mathrm{E}$ \\
\hline P08032 & Spectrin alpha chain, erythrocytic 1 OS=Mus musculus GN=Spta1 PE=1 SV=3 - [SPTA1_MOUSE] & $0,000 E_{0}$ & $0,000 \mathrm{E} 0$ & $0,000 \mathrm{E}^{\circ}$ & $5,728 E 6$ \\
\hline P59325 & Eukaryotic translation initiation factor $5 \mathrm{OS}=$ Mus musculus GN=Eif5 PE=1 SV=1 - [IF5_MOUSE] & $0,000 \mathrm{E} 0$ & $0,000 E 0$ & $0,000 \mathrm{E} 0$ & $1,703 \mathrm{E} 7$ \\
\hline Q9QXX4 & Calcium-binding mitochondrial carrier protein Aralar2 OS=Mus musculus GN=SIC25a13 PE=1 SV=1 - [CMC2_MOUSE] & $0,000 \mathrm{E}_{0}$ & $0,000 \mathrm{E} 0$ & $0,000 \mathrm{E}_{0}$ & $4,422 E 6$ \\
\hline P70452 & Syntaxin-4 OS=Mus musculus GN=Stx4 PE=1 SV=1 - [STX4_MOUSE] & $0,000 \mathrm{E} 0$ & $0,000 \mathrm{E} 0$ & $0,000 \mathrm{E} 0$ & $1,853 \mathrm{E} 7$ \\
\hline Q9D735 & Uncharacterized protein C19orf43 homolog OS=Mus musculus PE=1 SV=1 - [CSO43_MOUSE] & $0,000 \mathrm{E} 0$ & $0,000 \mathrm{E} 0$ & $0,000 \mathrm{E} 0$ & $2,063 \mathrm{E} 6$ \\
\hline Q91VC3 & Eukaryotic initiation factor 4A-III OS=Mus musculus GN=Eif4a3 PE=1 SV=3 - [IF4A3_MOUSE] & $0,000 \mathrm{E} 0$ & $0,000 \mathrm{E} 0$ & $0,000 \mathrm{E} 0$ & $1,511 \mathrm{E} 7$ \\
\hline Q7M6Y3-6 & Isoform 6 of Phosphatidylinositol-binding clathrin assembly protein OS=Mus musculus GN=Picalm - [PICAL_MOUSE] & $0,000 E 0$ & $0,000 E 0$ & $0,000 E_{0}$ & $5,366 \mathrm{E} 7$ \\
\hline 008553 & Dihydropyrimidinase-related protein 2 OS=Mus musculus GN=DpySI2 PE=1 SV=2 - [DPYL2_MOUSE] & $0,000 E 0$ & $0,000 E 0$ & $0,000 E 0$ & $3,094 \mathrm{E} 6$ \\
\hline Q99372-4 & Isoform 4 of DNA dC->dU-editing enzyme APOBEC-3 OS=Mus musculus GN=Apobec3 - [ABEC3_MOUSE] & $0,000 E 0$ & $0,000 E 0$ & $0,000 E_{0}$ & $1,687 \mathrm{E}$ \\
\hline P62835 & Ras-related protein Rap-1A OS=Mus musculus GN=Rap1a PE=1 SV=1 - [RAP1A_MOUSE] & $0,000 \mathrm{E} 0$ & $0,000 \mathrm{E} 0$ & $0,000 \mathrm{E} 0$ & $1,164 \mathrm{E} 7$ \\
\hline Q6PHZ2-2 & Isoform 2 of Calcium/calmodulin-dependent protein kinase type II subunit delta OS=Mus musculus GN=Camk2d - [KCC2D_MOUSE] & $0,000 E_{0}$ & $0,000 E_{0}$ & $0,000 E_{0}$ & $1,122 E 7$ \\
\hline Q3UMFO-3 & Isoform 3 of Cordon-bleu protein-like 1 OS=Mus musculus GN=Cobl11 - [COBL1_MOUSE] & $0,000 E_{0}$ & $0,000 E_{0}$ & $0,000 E_{0}$ & $5,329 \mathrm{E} 6$ \\
\hline P46938-2 & Isoform 2 of Transcriptional coactivator YAP1 OS=Mus musculus GN=Yap1 - [YAP1_MOUSE] & $0,000 E_{0}$ & $0,000 \mathrm{E} 0$ & $0,0000_{0}$ & $2,125 \mathrm{E} 7$ \\
\hline 008664 & B-cell CLL/ymphoma 7 protein family member C OS=Mus musculus GN=BC17c PE=1 SV=1 - [BCL7C_MOUSE] & $0,000 E 0$ & $0,000 E 0$ & $0,000 \mathrm{E} 0$ & 4,896E6 \\
\hline Q99ப0 & CTTNBP2 N-terminal-like protein OS=Mus musculus GN=Cttnbp2nI PE=1 SV=1 - [CT2NL_MOUSE] & $0,000 \mathrm{E} 0$ & $0,000 \mathrm{E} 0$ & $0,0000_{0}$ & $4,181 \mathrm{E} 6$ \\
\hline Q9CZT6 & Protein CMSS1 OS=Mus musculus GN=Cmss1 PE=2 SV=1 - [CMS1_MOUSE] & 0,000E0 & 0,000E0 & 0,000E0 & $4,121 \mathrm{E} 6$ \\
\hline 055131 & Septin-7 OS=Mus musculus GN=Sept7 PE=1 SV=1 - [SEPT7_MOUSE] & $0,000 E_{0}$ & $0,000 \mathrm{E} 0$ & $0,000 \mathrm{E}_{0}$ & $2,813 \mathrm{E}$ \\
\hline Q8CAQ8-2 & Isoform 2 of MICOS complex subunit Mic60 OS=Mus musculus GN=Immt - [MIC60_MOUSE] & $0,000 \mathrm{E} 0$ & $0,000 \mathrm{E} 0$ & $1,006 E 6$ & $7,826 \mathrm{E}$ \\
\hline 035608 & Angiopoietin-2 OS=Mus musculus GN=Angpt2 PE=2 SV=2 - [ANGP2_MOUSE] & $0,000 E 0$ & $0,000 E 0$ & $0,000 E_{0}$ & $1,818 \mathrm{E} 7$ \\
\hline Q8BHL4 & Retinoic acid-induced protein 3 OS=Mus musculus GN=Gprc5a PE=1 SV=1 - [RAI3_MOUSE] & $0,000 E 0$ & $0,000 E 0$ & $0,000 \mathrm{E} 0$ & 3,834E7 \\
\hline Q9CQ22 & Ragulator complex protein LAMTOR1 OS=Mus musculus GN=Lamtor1 PE=1 SV=1 - [LTOR1_MOUSE] & $0,000 E 0$ & $0,000 E 0$ & $0,000 \mathrm{E} 0$ & $5,700 \mathrm{E} 6$ \\
\hline Q8R1B4 & Eukaryotic translation initiation factor 3 subunit $C O S=$ Mus musculus GN=Eif3C PE $=1 \mathrm{SV}=1$ - [EIF3C_MOUSE] & $0,000 E 0$ & $0,000 \mathrm{E} 0$ & $1,214 E 6$ & $3,060 \mathrm{E} 7$ \\
\hline Q9CR57 & 60S ribosomal protein L14 OS=Mus musculus GN=Rp|14 PE=1 SV=3 - [RL14_MOUSE] & $0,000 E_{0}$ & $0,000 \mathrm{E} 0$ & $2,375 E 5$ & 9,775E7 \\
\hline Q9ROY5 & Adenylate kinase isoenzyme $10 \mathrm{OS}=$ Mus musculus GN=Ak1 PE=1 SV=1 - [KAD1_MOUSE] & $0,000 \mathrm{E} 0$ & $0,000 \mathrm{E} 0$ & $0,000 \mathrm{E} 0$ & $1,613 \mathrm{E} 6$ \\
\hline Q99PU8-2 & Isoform 2 of Putative ATP-dependent RNA helicase DHX30 OS=Mus musculus GN=DhX30 - [DHX30_MOUSE] & $0,000 \mathrm{E} 0$ & $0,000 \mathrm{E} 0$ & $0,000 \mathrm{E} 0$ & $6,139 \mathrm{E} 6$ \\
\hline Q8BK67 & Protein RCC2 OS=Mus musculus GN=Rcc2 PE=1 SV=1- [RCC2_MOUSE] & $0,000 \mathrm{E} 0$ & $0,000 \mathrm{E} 0$ & $0,000 \mathrm{E} 0$ & $1,435 \mathrm{E} 6$ \\
\hline Q9DC28 & Casein kinase I isoform delta OS=Mus musculus GN=Csnk1d PE=1 SV=2 - [KC1D_MOUSE] & $0,000 E 0$ & $0,000 E 0$ & $0,000 E 0$ & $1,802 E 7$ \\
\hline P21278 & Guanine nucleotide-binding protein subunit alpha-11 OS=Mus musculus GN=Gna11 PE=1 SV=1 - [GNA11_MOUSE] & $0,000 \mathrm{E} 0$ & $0,000 \mathrm{E} 0$ & $0,000 \mathrm{E} 0$ & $1,313 \mathrm{E} 7$ \\
\hline P35601-2 & Isoform 2 of Replication factor C subunit 1 OS=Mus musculus GN=RfC1 - [RFC1_MOUSE] & $0,000 \mathrm{E} 0$ & $0,000 \mathrm{E} 0$ & $5,241 E 5$ & $1,702 E 7$ \\
\hline Q08481 & Platelet endothelial cell achesion molecule OS=Mus musculus GN=Pecam1 PE=1 SV=1- [PECA1_MOUSE] & $0,000 \mathrm{E} 0$ & $0,000 \mathrm{E} 0$ & $0,000 \mathrm{E} 0$ & $1,680 \mathrm{E} 7$ \\
\hline Q9WVM1 & Rac GTPase-activating protein 1 OS=Mus musculus GN=Racgap1 PE=1 SV=1 - [RGAP1_MOUSE] & $0,000 E 0$ & $0,000 E 0$ & $0,000 E 0$ & 6,677E6 \\
\hline P13020-2 & Isoform 2 of Gelsolin OS=Mus musculus GN=Gsn - [GELS_MOUSE] & $0,000 \mathrm{E} 0$ & $0,000 E 0$ & $9,668 \mathrm{E} 6$ & $1,955 \mathrm{E} 7$ \\
\hline Q7M6Y3-5 & Isoform 5 of Phosphatidylinositol-binding clathrin assembly protein OS=Mus musculus GN=Picalm - [PICAL_MOUSE] & $0,000 \mathrm{E} 0$ & $0,000 \mathrm{E} 0$ & $0,000 E_{0}$ & 6,471E7 \\
\hline P63028 & Translationally-controlled tumor protein OS=Mus musculus GN=Tpt1 PE=1 SV=1 - [TCTP_MOUSE] & $0,000 E_{0}$ & $0,000 \mathrm{E} 0$ & $0,000 E_{0}$ & $6,326 \mathrm{E}$ \\
\hline Q99362 & Replication factor C subunit 4 OS=Mus musculus GN=Rfc4 PE=1 SV=1 - [RFC4_MOUSE] & $0,000 \mathrm{E} 0$ & $0,000 \mathrm{E} 0$ & $0,000 E_{0}$ & $3,254 \mathrm{E} 6$ \\
\hline Q6P542 & ATP-binding cassette sub-family F member $10 \mathrm{OS}=$ Mus musculus GN=Abcf1 PE=1 SV=1 - [ABCF1_MOUSE] & $0,000 \mathrm{E} 0$ & $0,000 \mathrm{E} 0$ & $0,000 \mathrm{E} 0$ & 2,163E7 \\
\hline Q9WTX2 & Interferon-inducible double-stranded RNA-dependent protein kinase activator A OS=Mus musculus GN=Prkra PE=1 SV=1 - [PRKRA_MOUSE] & $0,000 E 0$ & $0,000 \mathrm{E} 0$ & $0,000 E_{0}$ & $8,709 E 6$ \\
\hline P19157 & Glutathione S-transferase P 1 OS=Mus musculus GN=Gstp1 PE=1 SV=2 - [GSTP1_MOUSE] & $0,000 \mathrm{E} 0$ & $0,000 E 0$ & $0,000 \mathrm{E} 0$ & 6,291E6 \\
\hline Q1W617 & Protein Shroom4 OS=Mus musculus GN=Shroom 4 PE=1 SV=1 - [SHRM4_MOUSE] & $0,000 E 0$ & $0,000 E 0$ & $0,000 \mathrm{E} 0$ & $1,488 \mathrm{E} 7$ \\
\hline Q8K298 & Anillin $\mathrm{OS}=$ Mus musculus GN=Anln PE=1 SV=2 - [ANLN_MOUSE] & $0,000 \mathrm{E} 0$ & $0,000 \mathrm{E} 0$ & $0,000 \mathrm{E} 0$ & 6,823E6 \\
\hline Q8VH51-2 & Isoform 2 of RNA-binding protein 39 OS=Mus musculus GN=Rbm39 - [RBM39_MOUSE] & $1,010 \mathrm{E} 7$ & $0,000 \mathrm{E} 0$ & $2,877 E 6$ & $6,717 \mathrm{E}$ \\
\hline Q8VDR9 & Dedicator of cytokinesis protein 6 OS=Mus musculus GN=Dock6 PE=1 $\mathrm{SV}=4$ - [DOCK6_MOUSE] & 0,000E0 & $0,000 \mathrm{E} 0$ & 0,000E0 & $2,811 \mathrm{E}$ \\
\hline Q6A028 & Switch-associated protein 70 OS=Mus musculus GN=Swap70 PE=1 SV=2 - [SWP70_MOUSE] & $0,000 \mathrm{E} 0$ & $0,000 E 0$ & $0,000 \mathrm{E} 0$ & $7,522 E 6$ \\
\hline Q9D1R9 & 60S ribosomal protein L34 OS=Mus musculus GN=Rp134 PE=1 SV=2 - [RL34_MOUSE] & $0,000 \mathrm{E} 0$ & $0,000 \mathrm{E} 0$ & $0,000 \mathrm{E} 0$ & $9,653 \mathrm{E} 7$ \\
\hline Q9ESD7-2 & Isoform 2 of Dysferlin OS=Mus musculus GN=Dysf - [DYSF_MOUSE] & $0,000 E 0$ & $0,000 E 0$ & $0,000 E_{0}$ & $1,472 E 7$ \\
\hline P54775 & $26 \mathrm{~S}$ protease regulatory subunit $6 \mathrm{~B}$ OS=Mus musculus GN=PSmc4 PE=1 SV=2 - [PRS6B_MOUSE] & $0,0000_{0}$ & $0,000 \mathrm{E} 0$ & 9,752E5 & 3,711E6 \\
\hline Q64010-2 & Isoform Crk-I of Adapter molecule crk OS=Mus musculus GN=Crk - [CRK_MOUSE] & $0,000 E 0$ & $0,000 \mathrm{E} 0$ & $0,000 \mathrm{E} 0$ & $1,024 E 6$ \\
\hline Q60864 & Stress-induced-phosphoprotein 1 OS=Mus musculus GN=Stip1 PE=1 SV=1- [STIP1_MOUSE] & $0,000 \mathrm{E}_{0}$ & $0,000 \mathrm{E} 0$ & $0,000 \mathrm{E}_{0}$ & $5,908 E 6$ \\
\hline Q62465 & Synaptic vesicle membrane protein VAT-1 homolog OS=Mus musculus GN=Vat1 PE=1 SV=3 - [VAT1_MOUSE] & $0,000 \mathrm{E} 0$ & $0,000 \mathrm{E} 0$ & $0,000 \mathrm{E} 0$ & $1,740 \mathrm{E} 7$ \\
\hline Q9D0F6 & Replication factor C subunit 5 OS=Mus musculus GN=Rfc5 PE=1 SV=1 - [RFC5_MOUSE] & $0,000 \mathrm{E} 0$ & $0,000 \mathrm{E} 0$ & $0,000 \mathrm{E} 0$ & 6,434E6 \\
\hline Q8BMA3 & Connector enhancer of kinase suppressor of ras 3 OS=Mus musculus GN=Cnksr3 PE=1 SV=1- [CNKR3_MOUSE] & $0,000 \mathrm{E} 0$ & $0,000 \mathrm{E} 0$ & $0,000 \mathrm{E} 0$ & $3,575 \mathrm{E} 6$ \\
\hline Q76MZ3 & Serine/threonine-protein phosphatase 2A 65 kDa regulatory subunit A alpha isoform OS=Mus musculus GN=Ppp2r1a PE=1 SV=3 - [2AAA_MOUSE] & $0,000 E_{0}$ & $0,000 E 0$ & $0,000 E_{0}$ & $2,808 \mathrm{E} 7$ \\
\hline Q9ESD7 & Dysferlin OS=Mus musculus GN=Dysf PE=1 SV=3 - [DYSF_MOUSE] & $0,000 E 0$ & $0,000 E 0$ & $0,000 E 0$ & $1,472 E 7$ \\
\hline P52927 & High mobility group protein HMGI-C OS=Mus musculus GN=Hmga2 PE=1 SV=1 - [HMGA2_MOUSE] & $0,000 \mathrm{E} 0$ & $0,000 \mathrm{E} 0$ & $0,000 \mathrm{E} 0$ & $4,944 \mathrm{E} 7$ \\
\hline 070492 & Sorting nexin-3 OS=Mus musculus GN=Snx3 PE=1 SV=3 - [SNX3_MOUSE] & $0,000 \mathrm{E} 0$ & $0,000 \mathrm{E} 0$ & $0,000 \mathrm{E} 0$ & 7,197E5 \\
\hline P26883 & Peptidy-prolyl cis-trans isomerase FKBP1A OS=Mus musculus GN=Fkbp1a PE $=1 \mathrm{SV}=2$ - [FKB1A_MOUSE] & $0,000 E_{0}$ & $0,000 \mathrm{E} 0$ & $0,000 E_{0}$ & $1,798 \mathrm{E} 5$ \\
\hline P62852 & 40S ribosomal protein S25 OS=Mus musculus GN=Rps25 PE=1 SV=1 - [RS25_MOUSE] & $0,000 E 0$ & $0,000 E 0$ & $0,000 E 0$ & $4,270 \mathrm{E} 8$ \\
\hline P62743 & AP-2 complex subunit sigma OS=Mus musculus GN=AP2s1 PE=1 SV=1 - [AP2S1_MOUSE] & $0,000 \mathrm{E} 0$ & $0,000 \mathrm{E} 0$ & $0,0000_{0}$ & $1,235 E 8$ \\
\hline Q6NZK5 & Protein hinderin OS=Mus musculus GN=Kiaa1328 PE=1 SV=2 - [K1328_MOUSE] & $0,000 \mathrm{E} 0$ & $0,000 \mathrm{E} 0$ & $0,000 \mathrm{E} 0$ & $5,606 \mathrm{E} 6$ \\
\hline Q8R4U7 & Leucine zipper protein 1 OS=Mus musculus GN=Luzp1 PE=1 SV=2 - [LUZP1_MOUSE] & $0,000 \mathrm{E} 0$ & $0,000 \mathrm{E} 0$ & $0,0000_{0}$ & $5,260 E 6$ \\
\hline Q99\%85 & Phosphoserine aminotransferase OS=Mus musculus GN=Psat1 PE=1 SV=1 - [SERC_MOUSE] & $0,000 \mathrm{E} 0$ & $0,000 \mathrm{E} 0$ & $0,000 \mathrm{E} 0$ & $7,764 E 6$ \\
\hline Q9ES28-4 & Isoform D of Rho guanine nucleotide exchange factor 7 OS=Mus musculus GN=Arhgef7 - [ARHG7_MOUSE] & $0,000 E_{0}$ & $0,000 \mathrm{E} 0$ & $0,000 \mathrm{E}_{0}$ & 7,933E6 \\
\hline Q921Y4 & Thyroid receptor-interacting protein 6 OS=Mus musculus GN=Trip6 PE=1 SV=1 - [TRIP6_MOUSE] & $0,000 \mathrm{E} 0$ & $0,000 \mathrm{E} 0$ & $0,000 E_{0}$ & $4,312 E 6$ \\
\hline Q9D018 & mRNA turnover protein 4 homolog OS=Mus musculus GN=Mrto4 PE=1 SV=1 - [MRT4_MOUSE] & $0,000 E 0$ & $0,000 E 0$ & $0,000 E_{0}$ & $2,490 \mathrm{E} 6$ \\
\hline Q90937 & Uncharacterized protein C11orf98 homolog OS=Mus musculus PE=1 SV=1 - [CK098_MOUSE] & $0,000 E 0$ & $0,000 E 0$ & $0,000 \mathrm{E} 0$ & $2,049 \mathrm{E7}$ \\
\hline Q9CZY3 & Ubiquitin-conjugating enzyme E2 variant $1 \mathrm{OS}=$ Mus musculus GN=Ube2v1 PE=1 SV=1 - [UB2V1_MOUSE] & $0,000 \mathrm{E} 0$ & $0,000 \mathrm{E} 0$ & $0,000 \mathrm{E} 0$ & $5,127 \in 6$ \\
\hline Q993B8 & Protein kinase $\mathrm{C}$ and casein kinase II substrate protein 3 OS=Mus musculus $\mathrm{GN}=\mathrm{Pacsin} 3 \mathrm{PE}=1 \mathrm{SV}=1$ - [PACN3_MOUSE] & $0,000 E 0$ & $0,000 \mathrm{E} 0$ & $0,000 E_{0}$ & $1,013 \mathrm{E}$ \\
\hline P43274 & Histone H1.4 OS=Mus musculus GN=Hist1h1e PE=1 SV=2 - [H14_MOUSE] & $0,000 E_{0}$ & $0,000 E 0$ & $6,982 E 5$ & $5,100 \mathrm{E} 7$ \\
\hline Q99993 & Interferon-induced transmembrane protein $2 \mathrm{OS}=$ Mus musculus $\mathrm{GN}=$ Ifttm2 PE=1 $\mathrm{SV}=1$ - [IFM2_MOUSE] & $0,000 E 0$ & $0,000 E 0$ & $0,000 E_{0}$ & 3,591E6 \\
\hline Q62188 & Dihydropyrimidinase-related protein 3 OS=Mus musculus GN=Dpys33 PE=1 SV=1 - [DPYL3_MOUSE] & $0,000 \mathrm{E} 0$ & $0,000 \mathrm{E} 0$ & $0,000 \mathrm{E} 0$ & $4,926 \mathrm{E} 6$ \\
\hline Q04750 & DNA topoisomerase 1 OS=Mus musculus GN=Top1 PE=1 SV=2 - [TOP1_MOUSE] & $0,000 \mathrm{E} 0$ & $0,000 \mathrm{E} 0$ & $0,000 \mathrm{E} 0$ & $1,463 \mathrm{E} 7$ \\
\hline Q7TQHO-2 & Isoform 2 of Ataxin-2-like protein OS=Mus musculus GN=Atxn21 - [ATX2L_MOUSE] & $0,000 \mathrm{E} 0$ & $0,000 \mathrm{E} 0$ & $2,775 E 5$ & $2,831 \mathrm{E} 7$ \\
\hline Q99kY4 & Cyclin-G-associated kinase OS=Mus musculus GN=Gak PE=1 SV=2 - [GAK_MOUSE] & $0,000 \mathrm{E} 0$ & $0,000 \mathrm{E} 0$ & $0,000 \mathrm{E} 0$ & $3,046 \mathrm{E} 7$ \\
\hline Q62448-2 & Isoform 2 of Eukaryotic translation initiation factor 4 gamma 2 OS=Mus musculus GN=Eif4g2 - [IF4G__MOUSE] & $0,000 \mathrm{E} 0$ & $0,000 \mathrm{E} 0$ & $0,000 \mathrm{E}^{\circ}$ & $1,365 \mathrm{E} 7$ \\
\hline Q60634-3 & Isoform 3 of Flotillin-2 OS=Mus musculus GN=Flot2 - [FLOT2_MOUSE] & $0,000 \mathrm{E} 0$ & $0,000 \mathrm{E} 0$ & $0,000 \mathrm{E} 0$ & $7,771 \mathrm{E} 5$ \\
\hline P70441 & $\mathrm{Na}(+) / \mathrm{H}(+)$ exchange regulatory cofactor NHE-RF1 OS=Mus musculus $\mathrm{GN}=\mathrm{Slc9}$ a3r1 PE=1 $\mathrm{SV}=3$ - [NHRF1_MOUSE] & $0,000 \mathrm{E} 0$ & $0,000 E 0$ & $0,000 E_{0}$ & 3,953E6 \\
\hline P47955 & 60 a acidic ribosomal protein P1 OS=Mus musculus GN=Rplp1 PE=1 SV=1 - [RLA1_MOUSE] & $0,000 \mathrm{E} 0$ & $0,000 \mathrm{E} 0$ & $0,000 \mathrm{E} 0$ & $8,722 E 7$ \\
\hline P97479-2 & Isoform 2 of Unconventional myosin-VIIa OS=Mus musculus GN=Myo7a - [MYO7A_MOUSE] & $2,735 E 6$ & $0,000 \mathrm{E} 0$ & $0,000 \mathrm{E} 0$ & $1,253 \mathrm{E} 7$ \\
\hline Q9WV55 & Vesicle-associated membrane protein-associated protein A OS=Mus musculus GN=Vapa PE=1 $\mathrm{SV}=2$ - [VAPA_MOUSE] & $0,000 E_{0}$ & $0,000 \mathrm{E} 0$ & $0,000 E_{0}$ & $1,038 \mathrm{E} 7$ \\
\hline Q64727 & Vinculin OS=Mus musculus GN=VCl PE=1 SV=4 - [VINC_MOUSE] & $0,000 \mathrm{E} 0$ & $0,000 \mathrm{E} 0$ & $6,575 \mathrm{E} 6$ & $6,419 E 6$ \\
\hline Q8C310 & Roundabout homolog 4 OS=Mus musculus GN=Robo4 PE=1 SV=2 - [ROBO4_MOUSE] & $0,000 \mathrm{E} 0$ & $0,000 \mathrm{E} 0$ & $0,000 \mathrm{E} 0$ & $1,506 \mathrm{~F}$ \\
\hline Q60817 & eptide-associated co & $0,000 E 0$ & $0,000 \mathrm{E} 0$ & $0,000 E_{0}$ & $1,286 \mathrm{E} 8$ \\
\hline
\end{tabular}




\begin{tabular}{|c|c|c|c|c|c|}
\hline Q9WVR4 & Fragile X mental retardation syndrome-related protein 2 OS=Mus musculus GN=Fxr2 PE=1 SV=1 - [FXR2_MOUSE] & $0,000 \mathrm{E} 0$ & $0,000 \mathrm{E} 0$ & $0,000 E 0$ & $6,711 \mathrm{E} 7$ \\
\hline P45377 & Aldose reductase-related protein 2 OS=Mus musculus GN=Akr1b8 PE=1 SV=2 - [ALD2_MOUSE] & $0,000 \mathrm{E} 0$ & $0,000 \mathrm{E} 0$ & $0,000 E 0$ & $6,907 E 6$ \\
\hline Q9CQW1 & Synaptobrevin homolog YKT6 OS=Mus musculus GN=Ykt6 PE=1 SV=1 - [YKT6_MOUSE] & $0,000 E 0$ & $0,000 \mathrm{E} 0$ & $0,000 E 0$ & $2,513 \mathrm{E} 6$ \\
\hline Q8C1Q6 & Small integral membrane protein 4 OS=Mus musculus GN=Smim4 PE=1 SV=2 - [SMIM4_MOUSE] & $0,000 \mathrm{E} 0$ & $0,000 E 0$ & $0,000 E 0$ & $5,398 E 6$ \\
\hline Q8BVuo & Leucine-rich repeat and calponin homology domain-containing protein 3 OS=Mus musculus GN=Lrch3 PE=1 SV=3 - [LRCH3_MOUSE] & $0,000 E 0$ & $0,000 E 0$ & $0,000 E 0$ & $2,790 \mathrm{E} 7$ \\
\hline P11103 & Poly [ADP-ribose] polymerase $10 \mathrm{OS}=$ Mus musculus GN=Parp1 PE=1 SV=3 - [PARP1_MOUSE] & $0,000 \mathrm{E} 0$ & $0,000 \mathrm{E} 0$ & $0,000 E 0$ & 3,326E7 \\
\hline A2A758-2 & Isoform 2 of Uncharacterized protein KIAA1522 OS=Mus musculus GN=Kiaa1522 - [K1522_MOUSE] & $0,000 \mathrm{E} 0$ & $0,000 \mathrm{E} 0$ & $0,000 E 0$ & 4,083E6 \\
\hline 008967 & Cytohesin-3 OS=Mus musculus GN=Cyth3 PE=1 SV=1 - [CYH3_MOUSE] & $0,000 \mathrm{E} 0$ & $0,000 \mathrm{E} 0$ & $0,000 E 0$ & 9,632E6 \\
\hline QQGNC1 & Inverted formin-2 OS=Mus musculus GN=Inf2 PE=1 SV=1 - [INF2_MOUSE] & $0,000 \mathrm{E} 0$ & $0,000 \mathrm{E} 0$ & $0,000 E 0$ & $1,073 \mathrm{E}$ \\
\hline P55258 & Ras-related protein Rab-8A OS=Mus musculus GN=Rab8a PE=1 SV=2 - [RABBA_MOUSE] & $0,000 \mathrm{E} 0$ & $0,000 \mathrm{E} 0$ & $3,456 \mathrm{E5}$ & $8,538 \mathrm{E} 7$ \\
\hline P61028 & Ras-related protein Rab-8B OS=Mus musculus GN=Rab8b PE=1 SV=1 - [RABBB_MOUSE] & $0,000 \mathrm{E} 0$ & $0,000 \mathrm{E} 0$ & $3,456 E 5$ & $8,538 \mathrm{E} 7$ \\
\hline P10833 & Ras-related protein $R$-Ras OS=Mus musculus GN=Rras PE $=1 \mathrm{SV}=1-$ [RRAS_MOUSE] & $0,000 E_{0}$ & $0,000 \mathrm{E} 0$ & $0,000 E 0$ & $5,125 \mathrm{E} 7$ \\
\hline Q99KP6 & Pre-mRNA-processing factor 19 OS=Mus musculus GN=Prpf19 PE=1 SV=1 - [PRP19_MOUSE] & $0,000 \mathrm{E} 0$ & $0,000 E 0$ & $0,000 E 0$ & $1,126 \mathrm{E} 7$ \\
\hline Q8C310-2 & Isoform 2 of Roundabout homolog 4 OS=Mus musculus GN=Robo4 - [ROBO4_MOUSE] & $0,000 \mathrm{E}_{0}$ & $0,000 \mathrm{E} 0$ & $0,000 E 0$ & $1,233 E 7$ \\
\hline Q99J58 & PC4 and SFRS1-interacting protein OS=Mus musculus GN=Psip1 PE=1 SV=1 - [PSIP1_MOUSE] & $0,000 \mathrm{E} 0$ & $0,000 \mathrm{E} 0$ & $0,000 E 0$ & $4,153 \mathrm{E} 6$ \\
\hline 070318 & Band 4.1-like protein $2 \mathrm{OS}=$ Mus musculus GN=Epb4112 PE=1 $\mathrm{SV}=2$ - [E41L__MOUSE] & $0,000 \mathrm{E} 0$ & $0,000 \mathrm{E} 0$ & $0,000 E 0$ & $1,023 \mathrm{E} 7$ \\
\hline Q9Z1D1 & Eukaryotic translation initiation factor 3 subunit $G$ OS=Mus musculus GN=Eif3g PE=1 SV=2 - [EIF3G_MOUSE] & $0,000 \mathrm{E} 0$ & $0,000 \mathrm{E} 0$ & $0,000 \mathrm{EO}$ & $1,971 \mathrm{E} 7$ \\
\hline Q9JIK5 & Nucleolar RNA helicase 2 OS=Mus musculus GN=Ddx21 PE=1 SV=3 - [DDX21_MOUSE] & $0,000 E 0$ & $0,000 E 0$ & $0,000 E 0$ & 3,983E6 \\
\hline Q8R017 & Vacuolar protein sorting-associated protein 37B OS=Mus musculus GN=Vps37b PE=1 SV=1 - [VP37B_MOUSE] & $0,000 E 0$ & $0,000 E 0$ & $0,000 E 0$ & $6,840 E 6$ \\
\hline Q62425 & Cytochrome c oxidase subunit NDUFA4 OS=Mus musculus GN=Ndufa4 PE=1 $\mathrm{SV}=2$ - [NDUA4_MOUSE] & $0,000 E 0$ & $0,000 E 0$ & $0,000 E 0$ & $1,639 \mathrm{E} 7$ \\
\hline Q5DTX6 & Junctional protein associated with coronary artery disease OS $=$ Mus musculus GN=Jcad PE=1 SV=2 - [JCAD_MOUSE] & $0,000 \mathrm{E} 0$ & $0,000 \mathrm{E} 0$ & $0,000 E 0$ & $2,165 \mathrm{E} 6$ \\
\hline Q91ZU6-3 & Isoform 3 of Dystonin OS=Mus musculus GN=Dst - [DYST_MOUSE] & $0,000 E_{0}$ & $0,000 \mathrm{E} 0$ & $0,000 E 0$ & $1,372 E 7$ \\
\hline Q9D8Y0 & EF-hand domain-containing protein D2 OS=Mus musculus GN=Efhd2 PE=1 $\mathrm{SV}=1$ - [EFHD2_MOUSE] & $0,000 \mathrm{E} 0$ & $0,000 E 0$ & $0,000 E 0$ & $1,699 \mathrm{E} 6$ \\
\hline Q80X50-2 & Isoform 2 of Ubiquitin-associated protein 2-like OS=Mus musculus GN=Ubap21 - [UBP2L_MOUSE] & $0,000 E_{0}$ & $0,000 \mathrm{E} 0$ & $0,000 E 0$ & $2,248 E 7$ \\
\hline DOQMC3 & Myeloid cell nuclear differentiation antigen-like protein OS=Mus musculus $\mathrm{GN}=$ Mndal PE=1 SV=1 - [MNDAL_MOUSE] & $0,000 E 0$ & $0,000 E 0$ & $1,867 \pm 5$ & $9,720 \mathrm{E} 6$ \\
\hline G3X930 & Signal-induced proliferation-associated 1-like protein 3 OS=Mus musculus GN=Sipa113 PE=1 SV=1 - [SIL__MOUSE] & $0,000 \mathrm{E} 0$ & $0,000 \mathrm{E} 0$ & $0,000 E 0$ & $1,902 E 7$ \\
\hline P08207 & Protein S100-A10 OS=Mus musculus GN=S100a10 PE=1 SV=2 - [S10A__MOUSE] & 0,000E0 & 0,000E0 & $0,000 E 0$ & 9,947E6 \\
\hline Q9CR16 & Peptidyl-prolyl cis-trans isomerase D OS=Mus musculus GN=Ppid PE=1 SV=3 - [PPID_MOUSE] & $0,000 E_{0}$ & $0,000 \mathrm{E} 0$ & $0,000 E 0$ & $2,557,66$ \\
\hline P35550 & rRNA 2'-O-methyltransferase fibrillarin OS=Mus musculus GN=Fb PE=1 SV=2 - [FBRL_MOUSE] & $0,000 \mathrm{E} 0$ & $0,000 \mathrm{E} 0$ & $0,000 E 0$ & $9,037 \pm 6$ \\
\hline P56480 & ATP synthase subunit beta, mitochondrial OS=Mus musculus GN=Atp5b PE=1 SV=2 - [ATPB_MOUSE] & $0,000 E 0$ & $0,000 E 0$ & $0,000 E 0$ & $2,061 E 6$ \\
\hline Q8R1F1 & Niban-like protein 1 OS=Mus musculus GN=Fam129b PE=1 SV=2 - [NIBL1_MOUSE] & 0,000E0 & 0,000E0 & $0,000 E 0$ & 7,346E6 \\
\hline P10630 & Eukaryotic initiation factor 4A-II OS=Mus musculus GN=Eif4a2 PE=1 SV=2 - [IF4A2_MOUSE] & $0,000 \mathrm{E} 0$ & $0,000 \mathrm{E} 0$ & $3,565 E 5$ & $3,511 \mathrm{E} 7$ \\
\hline P97863 & Nuclear factor $1 \mathrm{~B}$-type OS=Mus musculus GN=Nfib PE=1 SV=2 - [NFIB_MOUSE] & $0,000 \mathrm{E} 0$ & $0,000 \mathrm{E} 0$ & $0,000 E 0$ & $1,739 \mathrm{E} 7$ \\
\hline 008585 & Clathrin light chain A OS=Mus musculus GN=CIta PE=1 SV=2 - [CLCA_MOUSE] & $0,000 E_{0}$ & $0,000 \mathrm{E} 0$ & $0,000 E 0$ & $1,618 \mathrm{~EB}$ \\
\hline P61089 & Ubiquitin-conjugating enzyme E2 N OS=Mus musculus GN=Ube2n PE=1 SV=1 - [UBE2N_MOUSE] & $0,000 \mathrm{E} 0$ & $0,000 \mathrm{E} 0$ & $0,000 E 0$ & $4,490 \mathrm{E} 6$ \\
\hline AZALU4 & Protein Shroom2 OS=Mus musculus GN=Shroom2 PE=1 SV=1 - [SHRM2_MOUSE] & $0,000 \mathrm{E} 0$ & $0,000 \mathrm{E} 0$ & $0,000 E 0$ & $3,672 \mathrm{E} 6$ \\
\hline P52293 & Importin subunit alpha-1 $1 \mathrm{OS}=$ Mus musculus GN=Kpna2 PE=1 SV=2 - [IMA1_MOUSE] & $0,000 \mathrm{E} 0$ & $0,000 \mathrm{E} 0$ & $0,000 E 0$ & 6,294E6 \\
\hline Q9DB77 & Cytochrome b-c1 complex subunit 2, mitochondrial OS=Mus musculus GN=Uqcrc2 PE=1 SV=1 - [QCR2_MOUSE] & $0,000 E 0$ & $0,000 E 0$ & $0,000 E 0$ & $1,047 \mathrm{E} 7$ \\
\hline Q7Tा50 & Serine/threonine-protein kinase MRCK beta OS=Mus musculus GN=Cdc42bpb PE=1 SV=2 - [MRCKB_MOUSE] & $0,000 \mathrm{E} 0$ & $0,000 \mathrm{E} 0$ & $0,000 E 0$ & $2,322 E 7$ \\
\hline P14685 & 265 proteasome non-ATPase regulatory subunit 3 OS=Mus musculus GN=Psmd3 PE=1 SV=3 - [PSMD3_MOUSE] & $0,000 \mathrm{E} 0$ & $0,000 \mathrm{E} 0$ & $0,000 E 0$ & $3,528 E 6$ \\
\hline P16460 & Argininosuccinate synthase OS=Mus musculus GN=Ass1 PE=1 $\mathrm{SV}=1$ - [ASSY_MOUSE] & $0,000 \mathrm{E} 0$ & $0,000 \mathrm{E} 0$ & $0,000 E 0$ & $1,045 E 6$ \\
\hline P56389 & Cytidine deaminase OS=Mus musculus $\mathrm{GN}=\mathrm{Cda}$ PE=1 SV=2 - [CDD_MOUSE] & 0,000E0 & 0,000E0 & $0,000 E 0$ & $1,768 \mathrm{E} 6$ \\
\hline P25976-2 & Isoform UBF2 of Nucleolar transcription factor 1 OS=Mus musculus GN=Ubtf - [UBF1_MOUSE] & $0,000 \mathrm{E} 0$ & $0,000 E 0$ & $0,000 E 0$ & $2,807 E 7$ \\
\hline P61226 & Ras-related protein Rap-2b OS=Mus musculus GN=Rap2b PE=1 SV=1 - [RAP2B_MOUSE] & $0,000 \mathrm{E} 0$ & $0,000 \mathrm{E} 0$ & $0,000 E 0$ & 6,647E6 \\
\hline Q9CQF3 & Cleavage and polyadenylation specificity factor subunit 5 OS=Mus musculus GN=Nudt21 PE=1 SV=1- [CPSF5_MOUSE] & $0,000 E_{0}$ & $0,000 \mathrm{E} 0$ & $0,000 E 0$ & 8,42966 \\
\hline $\mathrm{Q8C132}$ & BAG family molecular chaperone regulator 5 OS=Mus musculus GN=Bag5 PE=1 $1 \mathrm{SV}=1$ - [BAG5_MOUSE] & $0,000 E 0$ & $0,000 E 0$ & $0,000 E 0$ & \begin{tabular}{l|l|l}
$1,632 E 6$ \\
\end{tabular} \\
\hline Q8VI36 & Paxillin OS=Mus musculus GN=Pxn PE=1 SV=1 - [PAXI_MOUSE] & $0,000 E 0$ & $0,000 E 0$ & $0,000 E 0$ & $8,372 E 6$ \\
\hline Q9JL15 & Galectin-8 OS=Mus musculus GN=Lgals8 PE=1 SV=1 - [LEG8_MOUSE] & $0,000 E 0$ & $0,000 \mathrm{E} 0$ & $0,000 E 0$ & $8,521 \mathrm{E} 6$ \\
\hline P42567 & Epidermal growth factor receptor substrate 15 OS=Mus musculus GN=Eps15 PE=1 SV=1 - [EPS15_MOUSE] & $0,000 \mathrm{E} 0$ & $0,000 E 0$ & $0,000 E 0$ & $5,288 E 6$ \\
\hline Q9ES28-3 & Isoform C of Rho guanine nucleotide exchange factor 7 OS=Mus musculus GN=Arhgef7 - [ARHG7_MOUSE] & $0,000 E 0$ & $0,000 \mathrm{E} 0$ & $0,000 E 0$ & $7,933 E 6$ \\
\hline Q03963 & Interferon-induced, double-stranded RNA-activated protein kinase OS=Mus musculus GN=Eifzak2 PE=1 SV=2 - [E2AK2_MOUSE] & $0,000 \mathrm{E} 0$ & $0,000 \mathrm{E} 0$ & $0,000 E 0$ & $1,825 \mathrm{E} 7$ \\
\hline Q3TJZ6 & Protein FAM98A OS=Mus musculus GN=Fam98a PE=1 SV=1 - [FA98A_MOUSE] & $0,000 \mathrm{E} 0$ & $0,000 \mathrm{E} 0$ & $0,000 E 0$ & $1,311 \mathrm{E} 7$ \\
\hline Q9CPQ8 & ATP synthase subunit 9 , mitochondrial OS=Mus musculus GN=Atp5l PE=1 SV=1 - [ATPSL_MOUSE] & $0,000 \mathrm{E} 0$ & $0,000 E 0$ & $0,000 E 0$ & 7,494E6 \\
\hline Q9DBR1 & $5^{\prime}-33^{\prime}$ exoribonuclease 2 OS=Mus musculus GN=Xrn2 PE=1 SV=1 - [XRN2_MOUSE] & $0,000 \mathrm{E} 0$ & $0,000 E 0$ & $0,000 E 0$ & $1,271 E 7$ \\
\hline Q8BMJ3 & Eukaryotic translation initiation factor $1 \mathrm{~A}, \mathrm{X}$-chromosomal OS=Mus musculus $\mathrm{GN}=\mathrm{Eif1} 1 \mathrm{ax} \mathrm{PE}=2 \mathrm{SV}=3$ - [IF1AX_MOUSE] & $0,000 \mathrm{E} 0$ & $0,000 \mathrm{E} 0$ & $0,000 E 0$ & $5,414 \mathrm{E} 6$ \\
\hline Q5F2E8 & Serine/threonine-protein kinase TAO1 OS=Mus musculus GN=Taok1 PE=1 SV=1 - [TAOK1_MOUSE] & $0,000 \mathrm{E} 0$ & $0,000 \mathrm{E} 0$ & $8,146 E 5$ & $7,558 E 6$ \\
\hline Q921F4 & Heterogeneous nuclear ribonucleoprotein L-like OS=Mus musculus GN=Hnrnpll PE=1 SV=3 - [HNRLL_MOUSE] & $0,000 \mathrm{E} 0$ & $0,000 \mathrm{E} 0$ & $0,000 E 0$ & $7,243 E 6$ \\
\hline Q9DB34 & Charged multivesicular body protein 2a OS=Mus musculus GN=Chmp2a PE=1 SV=1 - [CHM2A_MOUSE] & $0,000 E 0$ & $0,000 E 0$ & $0,000 E 0$ & $1,985 E 7$ \\
\hline P49718 & DNA replication licensing factor MCM5 OS=Mus musculus GN=Mcm5 PE=1 SV=1 - [MCM5_MOUSE] & $0,000 \mathrm{E}_{0}$ & $0,000 \mathrm{E} 0$ & $0,000 E 0$ & $1,051 E 7$ \\
\hline P25206 & DNA replication licensing factor MCM3 OS=Mus musculus GN=Mcm3 PE=1 SV=2 - [MCM3_MOUSE] & $0,000 \mathrm{E} 0$ & $0,000 \mathrm{E} 0$ & $0,000 E 0$ & $6,610 \mathrm{E} 6$ \\
\hline 055135 & Eukaryotic translation initiation factor 6 OS=Mus musculus $\mathrm{GN}=\mathrm{E}$ Eif6 PE=1 SV=2 - [IF6_MOUSE] & $0,000 E 0$ & $0,000 \mathrm{E} 0$ & $0,000 E 0$ & $1,485 \mathrm{E} 7$ \\
\hline P28660-2 & Isoform 2 of Nck-associated protein 1 OS=Mus musculus GN=Nckap1 - [NCKP1_MOUSE] & $0,000 \mathrm{E} 0$ & $0,000 \mathrm{E} 0$ & 6,06115 & $2,268 \mathrm{E} 7$ \\
\hline Q8VIJ6 & Splicing factor, proline- and glutamine-rich OS=Mus musculus GN=Sfpq PE=1 $\mathrm{SV}=1$ - [SFPQ_MOUSE] & $0,000 E 0$ & $0,000 E 0$ & $0,000 E 0$ & 3,941E7 \\
\hline Q99kJ8 & Dynactin subunit 2 OS=Mus musculus GN=DCtn2 PE=1 SV=3 - [DCTN2_MOUSE] & $0,000 \mathrm{E} 0$ & $0,000 \mathrm{E} 0$ & $0,000 E 0$ & $1,506 \mathrm{E} 6$ \\
\hline Q8CBY8-2 & Isoform 2 of Dynactin subunit 4 OS=Mus musculus GN=Dctn4 - [DCTN4_MOUSE] & $0,000 \mathrm{E} 0$ & $0,000 \mathrm{E} 0$ & $0,000 E 0$ & $3,898 \mathrm{E} 6$ \\
\hline Q64012-2 & Isoform 1 of RNA-binding protein Raly OS=Mus musculus GN=Raly - [RALY_MOUSE] & $0,000 \mathrm{E} 0$ & $0,000 \mathrm{E} 0$ & $0,000 E 0$ & $1,885 \mathrm{E} 6$ \\
\hline Q91VR8 & Protein BRICK1 OS=Mus musculus GN=Brk1 PE=1 SV=1 - [BRK1_MOUSE] & $0,000 E_{0}$ & $0,000 \mathrm{E} 0$ & $0,000 E 0$ & $5,373 E 6$ \\
\hline Q92204-2 & Isoform C1 of Heterogeneous nuclear ribonucleoproteins $\mathrm{C} 1 / \mathrm{C} 2 \mathrm{OS}=$ Mus musculus GN=Hnrnpc - [HNRPC_MOUSE] & $0,000 \mathrm{E} 0$ & $0,000 E 0$ & $0,000 E 0$ & $5,070 \mathrm{E} 6$ \\
\hline Q8VEE4 & Replication protein A 70 kDa DNA-binding subunit OS=Mus musculus GN=Rpa1 PE=1 SV=1 - [RFA1_MOUSE] & $0,000 \mathrm{E} 0$ & $0,000 \mathrm{E} 0$ & $0,000 E 0$ & $4,744 E 6$ \\
\hline Q8R080 & 62 and $\mathrm{S}$ phase-expressed protein $1 \mathrm{OS}=$ Mus musculus $\mathrm{GN}=\mathrm{Gtse1} \mathrm{PE}=1 \mathrm{SV}=2$ - [GTSE1_MOUSE] & $0,000 \mathrm{E} 0$ & $0,000 \mathrm{E} 0$ & $0,000 E 0$ & $8,248 E 6$ \\
\hline Q9CX86 & Heterogeneous nuclear ribonucleoprotein $\mathrm{A0}$ OS=Mus musculus GN=Hnrnpa0 PE=1 SV=1- [ROA0_MOUSE] & $0,000 \mathrm{E} 0$ & $0,000 \mathrm{E} 0$ & $0,000 E 0$ & $1,633 \mathrm{E} 7$ \\
\hline P58137 & Acyl-coenzyme $A$ thioesterase 8 OS=Mus musculus GN=Acot8 PE=1 $\mathrm{SV}=1$ - [ACOT8_MOUSE] & 0,000E0 & 0,000E0 & $0,000 E 0$ & $4,770 \mathrm{E} 6$ \\
\hline P27601 & Guanine nucleotide-binding protein subunit alpha-13 OS=Mus musculus GN=Gna13 PE=1 SV=1 - [GNA13_MOUSE] & $0,000 E_{0}$ & $0,000 \mathrm{E} 0$ & $0,000 E 0$ & $2,341 \mathrm{E} 7$ \\
\hline Q80XC3 & USP6 N-terminal-like protein OS=Mus musculus GN=Usp6nl PE=1 SV=2 - [US6NL_MOUSE] & $0,000 \mathrm{E} 0$ & $0,000 \mathrm{E} 0$ & $0,000 E 0$ & $4,142 E 6$ \\
\hline Q8VDJ3 & Vigilin OS=Mus musculus GN=Hdlbp PE=1 SV=1 - [VIGLN_MOUSE] & $0,000 E 0$ & $0,000 E 0$ & $0,000 E 0$ & $5,970 E 6$ \\
\hline Q8CGU1 & Calcium-binding and coiled-coil domain-containing protein 1 OS=Mus musculus GN=Calcoco1 PE=1 SV=2 - [CACO1_MOUSE] & $0,000 E 0$ & $0,000 E 0$ & $0,000 E 0$ & \begin{tabular}{l|l|l}
$1,143 E 7$ \\
\end{tabular} \\
\hline Q66GT5 & Phosphatidylglycerophosphatase and protein-tyrosine phosphatase $10 \mathrm{OS}=$ Mus musculus GN=Ptpmt1 PE $=1 \mathrm{SV}=1$ - [PTPM1_MOUSE] & $0,000 \mathrm{E} 0$ & $0,000 \mathrm{E} 0$ & $0,000 E 0$ & $3,818 \mathrm{E} 6$ \\
\hline G5E829 & Plasma membrane calcium-transporting ATPase 1 OS=Mus musculus GN=Atp2b1 PE=1 SV=1 - [AT2B1_MOUSE] & $0,000 E 0$ & $0,000 \mathrm{E} 0$ & $0,000 E 0$ & $8,901 E 6$ \\
\hline Q60872 & Eukaryotic translation initiation factor $1 \mathrm{~A} O \mathrm{OS}=$ Mus musculus GN=Eif1a PE=2 SV=3 - [IF1A_MOUSE] & $0,000 E_{0}$ & $0,000 E 0$ & $0,000 E 0$ & $8,348 E 6$ \\
\hline Q9DB20 & ATP synthase subunit $\mathrm{O}$, mitochondrial OS=Mus musculus GN=Atp50 PE=1 SV=1 - [ATPO_MOUSE] & $0,000 E_{0}$ & $0,000 E 0$ & $0,000 E 0$ & $1,631 E 6$ \\
\hline Q8K124 & Pleckstrin homology domain-containing family O member 2 OS=Mus musculus GN=Plekho2 PE=1 SV=1 - [PKHO2_MOUSE] & $0,000 \mathrm{E} 0$ & $0,000 \mathrm{E} 0$ & $0,000 E 0$ & $2,216 \mathrm{E} 6$ \\
\hline Q03265 & ATP synthase subunit alpha, mitochondrial OS=Mus musculus GN=Atp5a1 PE=1 SV=1 - [ATPA_MOUSE] & $0,000 \mathrm{E} 0$ & $0,000 \mathrm{E} 0$ & 1,105E6 & $8,822 E 6$ \\
\hline Q60766-2 & Isoform 2 of Immunity-related GTPase family M protein 1 OS=Mus musculus GN=Irgm1 - [IRGM1_MOUSE] & $0,000 \mathrm{E} 0$ & $0,000 \mathrm{E} 0$ & $0,000 E 0$ & $9,248 E 6$ \\
\hline P56135 & ATP synthase subunit f, mitochondrial OS=Mus musculus $\mathrm{GN}=A \mathrm{Atp} 5 \mathrm{j} 2 \mathrm{PE}=1 \mathrm{SV}=3$ - [ATPK_MOUSE] & $0,000 \mathrm{E} 0$ & $0,000 \mathrm{E} 0$ & $0,000 E 0$ & $5,203 \mathrm{E} 6$ \\
\hline Q9WVA3 & Mitotic checkpoint protein BUB3 OS=Mus musculus GN=Bub3 PE=1 SV=2 - [BUB3_MOUSE] & $0,000 \mathrm{E} 0$ & $0,000 \mathrm{E} 0$ & $0,000 E 0$ & $6,979 E 6$ \\
\hline P54116 & Erythrocyte band 7 integral membrane protein OS=Mus musculus $\mathrm{GN}=\mathrm{Stom}$ PE=1 SV=3 - [STOM_MOUSE] & $0,000 \mathrm{E} 0$ & $0,000 E 0$ & $3,789 E 5$ & $2,246 \mathrm{E} 6$ \\
\hline P10107 & Annexin A1 OS=Mus musculus GN=Anxa1 PE=1 SV=2 - [ANXA1_MOUSE] & $0,000 \mathrm{E} 0$ & $0,000 E 0$ & $0,000 E 0$ & $1,019 E 7$ \\
\hline P25976 & Nucleolar transcription factor 1 OS=Mus musculus GN=Ubtf PE=1 SV=1 - [UBF1_MOUSE] & $0,000 \mathrm{E} 0$ & $0,000 \mathrm{E} 0$ & $0,000 E 0$ & $2,807 E 7$ \\
\hline Q8BYK6-3 & Isoform 3 of YTH domain-containing family protein 3 OS=Mus musculus GN=Ythdf3 - [YTHD3_MOUSE] & $0,000 \mathrm{E} 0$ & $0,000 \mathrm{E} 0$ & $5,369 \mathrm{E} 6$ & $1,804 \mathrm{E} 7$ \\
\hline Q9CRB9 & MICOS complex subunit Mic19 OS=Mus musculus GN=Chchd3 PE=1 SV=1 - [MIC19_MOUSE] & $0,000 E_{0}$ & $0,000 \mathrm{E}^{\circ}$ & $5,868 E 5$ & $8,755 E 6$ \\
\hline Q9Z0R6 & Intersectin-2 OS=Mus musculus GN=Itsn2 PE=1 SV=2 - [ITSN2_MOUSE] & $0,000 \mathrm{E} 0$ & $0,000 \mathrm{E} 0$ & $0,000 E 0$ & $1,868 \mathrm{E} 7$ \\
\hline Q9D1C2 & Protein chibby homolog 1 OS=Mus musculus GN=Cby1 PE=1 SV=1 - [CBY1_MOUSE] & $0,000 \mathrm{E} 0$ & $0,000 \mathrm{E} 0$ & $0,000 E 0$ & $1,215 \mathrm{E} 6$ \\
\hline Q910 & SGN=Rbms1 - [RBMS1_MOUSE] & $0,000 E 0$ & $0,000 \mathrm{E} 0$ & $0,000 E 0$ & $2,295 E 6$ \\
\hline
\end{tabular}




\begin{tabular}{|c|c|c|c|c|c|}
\hline Q8BPB0 & MOB kinase activator $1 \mathrm{~B}$ OS=Mus musculus GN=Mob1b PE=1 SV=3 - [MOB1B_MOUSE] & $0,000 \mathrm{E} 0$ & $0,000 \mathrm{E} 0$ & $0,000 \mathrm{E} 0$ & $1,913 \mathrm{E} 7$ \\
\hline P62827 & GTP-binding nuclear protein Ran OS=Mus musculus GN=Ran PE=1 SV=3 - [RAN_MOUSE] & $0,000 \mathrm{E} 0$ & $0,000 \mathrm{E} 0$ & $0,000 \mathrm{E} 0$ & $3,064 \mathrm{E} 7$ \\
\hline P26645 & Myristoylated alanine-rich C-kinase substrate $\mathrm{OS}=$ Mus musculus $\mathrm{GN}=$ Marcks PE=1 $\mathrm{SV}=2$ - [MARCS_MOUSE] & $0,000 E 0$ & $0,000 E 0$ & $0,000 \mathrm{E} 0$ & $4,771 \mathrm{E} 6$ \\
\hline 008579 & Emerin OS=Mus musculus GN=Emd PE=1 SV=1 - [EMD_MOUSE] & $0,000 \mathrm{E} 0$ & $0,000 E 0$ & $0,000 \mathrm{E} 0$ & 3,630E6 \\
\hline Q8VDZ4 & Palmitoyltransferase ZDHHC5 OS=Mus musculus GN=Zdhhc5 PE=1 SV=1 - [ZDHC5_MOUSE] & $0,000 E 0$ & $0,000 E 0$ & $0,000 E 0$ & $3,212 \mathrm{E} 6$ \\
\hline Q8C2Q3 & RNA-binding protein 14 OS=Mus musculus GN=Rbm14 PE=1 SV=1 - [RBM14_MOUSE] & $0,000 \mathrm{E} 0$ & $0,000 \mathrm{E} 0$ & $0,000 \mathrm{E} 0$ & $1,986 \mathrm{E} 7$ \\
\hline Q4VAA2-2 & Isoform 2 of Protein $\mathrm{CDV} 3 \mathrm{OS}=$ Mus musculus $\mathrm{GN}=\mathrm{Cdv} 3$ - [CDV3_MOUSE] & $0,000 \mathrm{E} 0$ & $0,000 \mathrm{E} 0$ & $0,000 \mathrm{E} 0$ & $1,485 \mathrm{E} 6$ \\
\hline P97477 & Aurora kinase A OS=Mus musculus GN=Aurka PE=1 SV=1 - [AURKA_MOUSE] & 0,000E0 & $0,000 \mathrm{E} 0$ & $0,000 \mathrm{E} 0$ & 1,045E6 \\
\hline Q9CQM9 & Glutaredoxin-3 OS=Mus musculus GN=Glrx3 PE=1 SV=1 - [GLRX3_MOUSE] & $0,000 \mathrm{E} 0$ & $0,000 E 0$ & $0,000 \mathrm{E} 0$ & $9,684 \mathrm{E} 6$ \\
\hline P35831 & Tyrosine-protein phosphatase non-receptor type 12 OS=Mus musculus GN=Ptpn12 PE=1 SV=3 - [PTN12_MOUSE] & $0,000 \mathrm{E} 0$ & $0,000 \mathrm{E} 0$ & $0,000 \mathrm{E} 0$ & $3,444 \mathrm{E} 6$ \\
\hline Q62418-3 & Isoform 3 of Drebrin-like protein OS=Mus musculus GN=Dbnl - [DBNL_MOUSE] & $0,000 \mathrm{E} 0$ & $0,000 E 0$ & $0,000 E_{0}$ & $8,414 E 6$ \\
\hline 054916 & RalBP1-associated Eps domain-containing protein 1 OS=Mus musculus GN=Reps1 PE=1 SV=2 - [REPS1_MOUSE] & $0,000 E_{0}$ & $0,000 \mathrm{E} 0$ & $0,000 \mathrm{E}^{\circ}$ & $1,497 \mathrm{E}$ \\
\hline Q91VI7 & Ribonuclease inhibitor OS=Mus musculus GN=Rnh1 PE=1 SV=1 - [RINL_MOUSE] & $0,000 \mathrm{E} 0$ & $0,000 E 0$ & $0,000 \mathrm{E} 0$ & $1,471 E 7$ \\
\hline P47915 & $60 S$ ribosomal protein $L 29$ OS=Mus musculus GN=Rp129 PE=2 SV=2 - [RL29_MOUSE] & $5,786 \mathrm{E} 6$ & $0,000 \mathrm{E} 0$ & $0,000 \mathrm{E}_{0}$ & $1,387 \mathrm{E} 8$ \\
\hline P32067 & Lupus La protein homolog OS=Mus musculus GN=Ssb PE=1 SV=1 - [LA_MOUSE] & $0,000 \mathrm{E} 0$ & $0,000 \mathrm{E} 0$ & $0,000 \mathrm{E} 0$ & $7,627 E 6$ \\
\hline Q9QWT9 & Kinesin-like protein KIFC1 OS=Mus musculus GN=Kifc1 PE=1 SV=2 - [KIFC1_MOUSE] & $0,000 \mathrm{E} 0$ & $0,000 \mathrm{E} 0$ & $0,000 \mathrm{E} 0$ & $6,150 \mathrm{E} 6$ \\
\hline Q02053 & Ubiquitin-like modifier-activating enzyme 1 OS=Mus musculus GN=Uba1 PE=1 $\mathrm{SV}=1$ - [UBA1_MOUSE] & $0,000 \mathrm{E} 0$ & $0,000 \mathrm{E} 0$ & $0,000 \mathrm{E} 0$ & $1,124 \mathrm{E} 7$ \\
\hline Q8BX02 & KN motif and ankyrin repeat domain-containing protein $2 \mathrm{OS}=$ Mus musculus $\mathrm{GN}=$ Kank2 PE=1SV=1 - [KANK2_MOUSE] & $0,000 E 0$ & $0,000 E 0$ & $0,000 E_{0}$ & $3,546 \mathrm{E} 6$ \\
\hline E9Q6B2 & Coiled-coil domain-containing protein $85 \mathrm{C}$ OS=Mus musculus $\mathrm{GN}=\mathrm{Ccdc} 85 \mathrm{C} \mathrm{PE}=1 \mathrm{SV}=1-$ [CC85C_MOUSE] & $0,000 E 0$ & $0,000 E 0$ & $0,000 E 0$ & $1,008 E 7$ \\
\hline Q69238 & Pseudopodium-enriched atypical kinase 1 OS=Mus musculus GN=Peak1 PE=1 SV=4 - [PEAK1_MOUSE] & $0,000 E 0$ & $0,000 E 0$ & $0,000 E_{0}$ & $8,664 E 6$ \\
\hline P01942 & Hemoglobin subunit alpha OS=Mus musculus GN=Hba PE=1 SV=2 - [HBA_MOUSE] & $8,540 \mathrm{E} 7$ & $0,000 \mathrm{E} 0$ & $0,000 \mathrm{E} 0$ & $3,162 \mathrm{E} 6$ \\
\hline Q99KD5 & Protein unc-45 homolog A OS=Mus musculus GN=Unc45a PE=1 $\mathrm{SV}=2$ - [UN45A_MOUSE] & $0,000 E_{0}$ & $0,000 \mathrm{E} 0$ & $0,000 E_{0}$ & $1,333 \mathrm{E} 7$ \\
\hline 035326 & Serine/arginine-rich splicing factor 5 OS=Mus musculus GN=Srsf5 PE=1 SV=2 - [SRSF5_MOUSE] & $0,000 \mathrm{E} 0$ & $0,000 E 0$ & $0,000 \mathrm{E} 0$ & $5,935 \mathrm{E} 7$ \\
\hline Q8R081 & Heterogeneous nuclear ribonucleoprotein L OS=Mus musculus $\mathrm{GN}=\mathrm{Hnrnpl}$ PE=1 SV=2 - [HNRPL_MOUSE] & $0,000 E_{0}$ & $0,000 \mathrm{E} 0$ & $0,0000_{0}$ & $5,301 E 6$ \\
\hline Q8K4G5-2 & Isoform 2 of Actin-binding LIM protein 1 OS=Mus musculus GN=Ablim1 - [ABLM1_MOUSE] & $0,000 E 0$ & $0,000 E 0$ & $0,000 E 0$ & $1,459 \mathrm{E} 7$ \\
\hline Q9D6T0 & Nitric oxide synthase-interacting protein OS=Mus musculus GN=Nosip PE=1 SV=1 - [NOSIP_MOUSE] & $0,000 \mathrm{E} 0$ & $0,000 \mathrm{E} 0$ & $0,0000_{0}$ & $2,716 \mathrm{E} 6$ \\
\hline Q3UHK8 & Trinucleotide repeat-containing gene $6 \mathrm{~A}$ protein $\mathrm{OS}=$ Mus musculus $\mathrm{GN}=\mathrm{Tnrc6a} \mathrm{PE}=2 \mathrm{SV}=1$ - [TNR6A_MOUSE] & $0,000 \mathrm{E} 0$ & $0,000 \mathrm{E} 0$ & $0,000 \mathrm{E} 0$ & $1,113 \mathrm{E} 7$ \\
\hline Q5NBX1-3 & Isoform 3 of Protein cordon-bleu OS=Mus musculus GN=Cobl - [COBL_MOUSE] & $0,000 \mathrm{E} 0$ & $0,000 \mathrm{E} 0$ & $0,000 \mathrm{E} 0$ & $3,065 E 6$ \\
\hline Q9Z0P5-2 & Isoform 2 of Twinflin-2 OS=Mus musculus GN=Twf2 - [TWF2_MOUSE] & $0,000 \mathrm{E} 0$ & $0,000 \mathrm{E} 0$ & $0,000 E_{0}$ & $2,270 E 6$ \\
\hline P81270-4 & Isoform 5 of Transcriptional regulator ERG OS=Mus musculus GN=Erg - [ERG_MOUSE] & $0,000 E 0$ & $0,000 E 0$ & $0,000 E_{0}$ & $1,590 \mathrm{E} 7$ \\
\hline Q9DCJ5 & NADH dehydrogenase [ubiquinone] 1 alpha subcomplex subunit 8 OS=Mus musculus GN=Ndufa8 PE=1 SV=3 - [NDUA8_MOUSE] & $0,000 \mathrm{E} 0$ & $0,000 \mathrm{E} 0$ & $0,000 \mathrm{E} 0$ & $5,153 \mathrm{E} 6$ \\
\hline Q9CX84 & Regulator of G-protein signaling 19 OS=Mus musculus GN=Rgs19 PE=1 SV=1 - [RGS19_MOUSE] & $0,000 \mathrm{E} 0$ & $0,000 \mathrm{E} 0$ & $0,000 \mathrm{E} 0$ & $2,998 \mathrm{E} 6$ \\
\hline P24452 & Macrophage-capping protein OS=Mus musculus GN=Capg PE=1 SV=2 - [CAPG_MOUSE] & $0,000 \mathrm{E} 0$ & $0,000 \mathrm{E} 0$ & $0,000 \mathrm{E} 0$ & $1,727 \mathrm{E} 7$ \\
\hline P17156 & Heat shock-related $70 \mathrm{kDa}$ protein $2 \mathrm{OS}=$ Mus musculus $\mathrm{GN}=\mathrm{Hspa2} \mathrm{PE}=1 \mathrm{SV}=2$ - [HSP72_MOUSE] & $3,628 \mathrm{E7}$ & $0,000 E_{0}$ & 9,603E6 & $5,026 \mathrm{E} 8$ \\
\hline Q8BT18-3 & Isoform 3 of Serine/arginine repetitive matrix protein 2 OS=Mus musculus GN=Srrm2 - [SRRM2_MOUSE] & $0,000 \mathrm{E} 0$ & $0,000 \mathrm{E}$ & $0,000 \mathrm{E}$ & 6,293E7 \\
\hline Q999R5 & Tubulointerstitial nephritis antigen-like OS=Mus musculus GN=Tinagl1 PE=1 SV=1 - [TINAL_MOUSE] & $0,000 \mathrm{E} 0$ & $0,000 \mathrm{E} 0$ & $0,000 \mathrm{E} 0$ & $2,047 \mathrm{E}$ \\
\hline Q8CHU3-2 & Isoform 2 of Epsin-2 OS=Mus musculus GN=Epn2 - [EPN2_MOUSE] & $0,000 \mathrm{E} 0$ & $0,000 \mathrm{E} 0$ & $0,000 \mathrm{E} 0$ & $1,593 \mathrm{E} 7$ \\
\hline Q9R0P4 & Small acidic protein OS=Mus musculus GN=Smap PE=1 SV=1 - [SMAP_MOUSE] & $0,000 E 0$ & $0,000 E 0$ & $0,000 E 0$ & $1,453 \mathrm{E} 7$ \\
\hline Q8K2Q7 & BRO1 domain-containing protein BROX OS=Mus musculus GN=Brox PE=1 SV=1 - [BROX_MOUSE] & $0,000 \mathrm{E} 0$ & $0,000 \mathrm{E} 0$ & $0,000 \mathrm{E} 0$ & $1,442 E 7$ \\
\hline Q8CIN4 & Serine/threonine-protein kinase PAK 2 OS=Mus musculus GN=Pak2 PE=1 SV=1 - [PAK2_MOUSE] & $0,000 \mathrm{E} 0$ & $0,000 \mathrm{E} 0$ & $0,000 \mathrm{E}^{\circ}$ & $2,749 \mathrm{E} 6$ \\
\hline 035286 & Pre-mRNA-splicing factor ATP-dependent RNA helicase DHX15 OS=Mus musculus GN=Dhx15 PE=1 SV=2 - [DHX15_MOUSE] & $0,000 \mathrm{E} 0$ & $0,000 \mathrm{E} 0$ & $0,000 \mathrm{E} 0$ & $1,157 \mathrm{E} 7$ \\
\hline Q63844 & Mitogen-activated protein kinase 3 OS=Mus musculus GN=Mapk3 PE=1 $\mathrm{SV}=5$ - [MK03_MOUSE] & $0,000 \mathrm{E} 0$ & $0,000 E 0$ & $0,000 E 0$ & $7,220 \mathrm{E} 6$ \\
\hline Q3UL36 & Arginine and glutamate-rich protein 1 OS=Mus musculus GN=Arglu1 PE=1 $\mathrm{SV}=2$ - [ARGL1_MOUSE] & $0,000 \mathrm{E} 0$ & $0,000 E 0$ & $0,000 \mathrm{E} 0$ & $2,188 \mathrm{E} 7$ \\
\hline Q9WVE8 & Protein kinase $\mathrm{C}$ and casein kinase substrate in neurons protein $2 \mathrm{OS}=$ Mus musculus GN=Pacsin2 PE=1SV=1 - [PACN2_MOUSE] & $0,000 \mathrm{E} 0$ & $0,000 \mathrm{E} 0$ & $0,000 \mathrm{E} 0$ & $8,708 E 6$ \\
\hline 035682 & Myeloid-associated differentiation marker OS=Mus musculus GN=Myadm PE=1 SV=2 - [MYADM_MOUSE] & $0,000 E_{0}$ & $0,000 \mathrm{E} 0$ & $0,000 E_{0}$ & $1,847 \mathrm{E} 7$ \\
\hline 070251 & Elongation factor 1-beta OS=Mus musculus GN=Eef1b PE=1 SV=5 - [EF1B_MOUSE] & $0,000 E 0$ & $0,000 E 0$ & $0,000 E 0$ & \begin{tabular}{l|l}
$8,634 E 6$ \\
\end{tabular} \\
\hline Q922Q8 & Leucine-rich repeat-containing protein 59 OS=Mus musculus GN=Lrrc59 PE=1 SV=1 - [LRC59_MOUSE] & $0,000 E 0$ & $0,000 E 0$ & $0,000 E 0$ & $1,407 E 7$ \\
\hline Q8C1B5 & Fermitin family homolog 2 OS=Mus musculus GN=Fermt2 PE=1 SV=1 - [FERM2_MOUSE] & $0,000 E 0$ & $0,000 \mathrm{E} 0$ & $0,000 E_{0}$ & $5,421 E 6$ \\
\hline Q8BRV5 & Uncharacterized protein KIAA1671 OS=Mus musculus GN=Kiaa1671 PE=2 SV=1 - [K1671_MOUSE] & $0,000 \mathrm{E} 0$ & $0,000 E 0$ & $0,000 \mathrm{E} 0$ & 3,164E6 \\
\hline Q3UV16 & Inositol 1,4,5--trisphosphate receptor-interacting protein-like $2 \mathrm{OS}=$ Mus musculus GN=Itpripl2 PE=2 SV=1 - [IPIL2_MOUSE] & $0,000 E 0$ & $0,000 E 0$ & $0,000 \mathrm{E} 0$ & 7,271E6 \\
\hline Q8CD15 & Bifunctional Iysine-specific demethylase and histidy-hydroxylase MINA OS=Mus musculus GN=Mina PE=1SV=2 - [MINA_MOUSE] & $0,000 \mathrm{E} 0$ & $0,000 \mathrm{E} 0$ & $0,000 \mathrm{E} 0$ & 6,348E6 \\
\hline Q99M87-2 & Isoform 2 of DnaJ homolog subfamily A member 3, mitochondrial OS=Mus musculus GN=Dnaja3 - [DNJA3_MOUSE] & $0,000 \mathrm{E} 0$ & $0,000 \mathrm{E} 0$ & $0,000 \mathrm{E} 0$ & $4,330 \mathrm{E} 6$ \\
\hline Q3TLH4 & Protein PRRC2C OS=Mus musculus GN=Prrc2C PE=1 SV=3 - [PRC2C_MOUSE] & 0,000E0 & $0,000 \mathrm{E} 0$ & $0,000 \mathrm{E} 0$ & $2,502 E 7$ \\
\hline Q6PGC1 & ATP-dependent RNA helicase DHX29 OS=Mus musculus GN=Dhx29 PE=1 SV=1 - [DHX29_MOUSE] & $0,000 \mathrm{E} 0$ & $0,000 E 0$ & $0,000 \mathrm{E} 0$ & 7,366E6 \\
\hline Q8BZIO & Actin filament-associated protein 1-like 1 OS=Mus musculus GN=Afap111 PE=1 SV=1 - [AF1L1_MOUSE] & $0,000 \mathrm{E} 0$ & $0,000 \mathrm{E} 0$ & $0,000 \mathrm{E} 0$ & $2,343 E 6$ \\
\hline Q91W53 & Golgin subfamily A member 7 OS=Mus musculus GN=Golga7 PE=1 SV=1 - [GOGA7_MOUSE] & $0,000 \mathrm{E} 0$ & $0,000 E 0$ & $0,000 E_{0}$ & $5,104 E 6$ \\
\hline Q811D0-2 & Isoform 2 of Disks large homolog 1 OS=Mus musculus GN=Dlg1 - [DLG1_MOUSE] & $0,000 \mathrm{E} 0$ & $0,000 \mathrm{E} 0$ & $0,000 \mathrm{E}^{\circ}$ & $5,706 \mathrm{E} 6$ \\
\hline 008917 & Flotillin-1 OS=Mus musculus GN=Flot1 PE=1 SV=1 - [FLOT1_MOUSE] & $0,000 E 0$ & $0,000 E 0$ & $0,000 E_{0}$ & $6,363 \mathrm{E} 6$ \\
\hline Q05793 & Basement membrane-specific heparan sulfate proteoglycan core protein OS=Mus musculus GN=Hspg2 PE=1 SV=1 - [PGBM_MOUSE] & $0,000 \mathrm{E}_{0}$ & $0,000 \mathrm{E} 0$ & $2,843 E 5$ & 4,050E7 \\
\hline Q04899 & Cyclin-dependent kinase 18 OS=Mus musculus $\mathrm{GN}=\mathrm{Cdk18} \mathrm{PE}=1 \mathrm{SV}=1$ - [CDK18_MOUSE] & $0,000 \mathrm{E} 0$ & $0,000 \mathrm{E} 0$ & $0,000 \mathrm{E} 0$ & $1,843 E 7$ \\
\hline Q9R1P4 & Proteasome subunit alpha type-1 $\mathrm{OS}=$ Mus musculus GN=Psma1 PE=1 $\mathrm{SV}=1$ - [PSA1_MOUSE] & $0,000 E 0$ & $0,000 \mathrm{E} 0$ & $0,000 E 0$ & $2,894 E 6$ \\
\hline P97762 & Retinitis pigmentosa 9 protein homolog OS=Mus musculus GN=rp9 PE=1 SV=1 - [RP9_MOUSE] & $0,000 \mathrm{E} 0$ & $0,000 \mathrm{E} 0$ & $0,000 \mathrm{E} 0$ & 1,814E7 \\
\hline Q61024 & Asparagine synthetase [glutamine-hydrolyzing] OS=Mus musculus GN=Asns PE=1 SV=3 - [ASNS_MOUSE] & $0,000 E_{0}$ & $0,000 E 0$ & $0,000 E_{0}$ & 4,58556 \\
\hline Q60780-2 & Isoform 2 of Growth arrest-specific protein 7 OS=Mus musculus GN=Gas7 - [GAS7_MOUSE] & $0,000 E 0$ & $0,000 E 0$ & $0,000 E 0$ & $4,506 \mathrm{E} 6$ \\
\hline P47964 & $60 S$ ribosomal protein $\mathrm{L36}$ OS=Mus musculus GN=Rp136 PE=3 SV=2 - [RL36_MOUSE] & $0,000 \mathrm{E} 0$ & $0,000 \mathrm{E} 0$ & $0,000 \mathrm{E} 0$ & $5,638 \mathrm{E} 7$ \\
\hline Q9EQK5 & Major vault protein OS=Mus musculus GN=Mvp PE=1 SV=4 - [MVP_MOUSE] & $0,000 \mathrm{E} 0$ & $0,000 \mathrm{E} 0$ & $0,000 \mathrm{E} 0$ & $5,282 E 6$ \\
\hline P23249 & Putative helicase MOV-10 OS=Mus musculus GN=Mov10 PE=1 SV=2 - [MOV10_MOUSE] & $0,000 E_{0}$ & $0,000 \mathrm{E} 0$ & $0,000 E_{0}$ & $9,086 \mathrm{E} 6$ \\
\hline 008784 & Treacle protein OS=Mus musculus GN=Tcof1 PE=1 SV=1 - [TCOF_MOUSE] & $0,000 \mathrm{E} 0$ & $0,000 E 0$ & $0,000 \mathrm{E} 0$ & 9,476E6 \\
\hline 088456 & Calpain small subunit 1 OS=Mus musculus GN=Capns1 PE=1 SV=1 - [CPNS1_MOUSE] & $0,000 \mathrm{E} 0$ & $0,000 \mathrm{E} 0$ & $0,000 \mathrm{E}^{\circ}$ & $1,073 E 7$ \\
\hline Q9QZS3-4 & Isoform 4 of Protein numb homolog OS=Mus musculus GN=Numb - [NUMB_MOUSE] & $0,000 \mathrm{E} 0$ & $0,000 \mathrm{E} 0$ & $0,000 \mathrm{E} 0$ & $1,424 \mathrm{E} 7$ \\
\hline Q6NZJ6 & Eukaryotic translation initiation factor 4 gamma 1 OS=Mus musculus GN=Eif4g1 PE=1 SV=1 - [IF4G1_MOUSE] & $0,000 \mathrm{E} 0$ & $0,000 \mathrm{E} 0$ & $0,0000_{0}$ & $2,752 E 7$ \\
\hline Q80X41-2 & Isoform 2 of Serine/threonine-protein kinase VRK1 OS=Mus musculus GN=Vrk1 - [VRK1_MOUSE] & $0,000 \mathrm{E} 0$ & $0,000 \mathrm{E} 0$ & $0,000 \mathrm{E} 0$ & $3,237 E 6$ \\
\hline Q62130 & Tyrosine-protein phosphatase non-receptor type 14 OS=Mus musculus GN=Ptpn14 PE=1 SV=2 - [PTN14_MOUSE] & $0,000 E_{0}$ & $0,000 \mathrm{E} 0$ & $0,000 \mathrm{E}_{0}$ & $4,449 \mathrm{E} 6$ \\
\hline 055222 & Integrin-linked protein kinase OS=Mus musculus GN=Ilk PE=1 SV=2 - [ILK_MOUSE] & $0,000 \mathrm{E} 0$ & $0,000 \mathrm{E} 0$ & $0,000 E_{0}$ & $6,128 E 6$ \\
\hline Q9JM52-3 & Isoform 3 of Misshapen-like kinase 1 OS=Mus musculus GN=Mink1 - [MINK1_MOUSE] & $0,000 E 0$ & $0,000 E 0$ & $0,000 E_{0}$ & $1,194 \mathrm{E} 7$ \\
\hline Q05D44 & Eukaryotic translation initiation factor 5B OS=Mus musculus GN=Eif5b PE=1 SV=2 - [IF2P_MOUSE] & $0,000 \mathrm{E} 0$ & $0,000 E 0$ & $0,000 \mathrm{E}_{0}$ & $1,555 \mathrm{E} 7$ \\
\hline Q61792 & LIM and SH3 domain protein 1 OS=Mus musculus GN=Lasp1 PE=1 SV=1 - [LASP1_MOUSE] & $0,000 \mathrm{E} 0$ & $0,000 \mathrm{E} 0$ & 1,221E6 & $1,556 \mathrm{E} 7$ \\
\hline P03975 & IgE-binding protein OS=Mus musculus GN=Iap PE=2 SV=1 - [IGEB_MOUSE] & $0,000 \mathrm{E} 0$ & $0,000 \mathrm{E} 0$ & $0,000 \mathrm{E} 0$ & $2,446 \mathrm{E} 7$ \\
\hline Q9Z0P4 & Paralemmin-1 OS=Mus musculus GN=Palm PE=1 SV=1 - [PALM_MOUSE] & $0,000 E_{0}$ & $0,000 E 0$ & $0,000 E_{0}$ & 1,69766 \\
\hline POCOA3 & Charged multivesicular body protein 6 OS=Mus musculus $\mathrm{GN}=\mathrm{Chmp6} \mathrm{PE}=1 \mathrm{SV}=2$ - [CHMP6_MOUSE] & $0,000 \mathrm{E} 0$ & $0,000 \mathrm{E} 0$ & $0,000 \mathrm{E} 0$ & 3,477E6 \\
\hline P06745 & Glucose-6-phosphate isomerase OS=Mus musculus GN=Gpi PE=1 SV=4 - [G6PI_MOUSE] & $0,000 \mathrm{E} 0$ & $0,000 \mathrm{E} 0$ & $0,000 \mathrm{E} 0$ & $1,591 \mathrm{E} 6$ \\
\hline 070152 & Dolichol-phosphate mannosyltransferase subunit $1 \mathrm{OS}=$ Mus musculus GN=Dpm1 PE=1 $\mathrm{SV}=1$ - [DPM1_MOUSE] & $0,000 \mathrm{E} 0$ & $0,000 \mathrm{E} 0$ & $0,000 \mathrm{E} 0$ & 3,099E6 \\
\hline P46467 & Vacuolar protein sorting-associated protein $4 \mathrm{~B}$ OS=Mus musculus $\mathrm{GN}=\mathrm{VPS} 4 \mathrm{~b} \mathrm{PE}=1 \mathrm{SV}=2$ - [VPS4B_MOUSE] & $0,000 \mathrm{E}$ & $0,000 \mathrm{E}$ & $0,000 \mathrm{E}$ & $1,535 \mathrm{E} 7$ \\
\hline Q9WVJ2 & 265 proteasome non-ATPase regulatory subunit 13 OS=Mus musculus GN=Psmd13 PE=1 SV=1 - [PSD13_MOUSE] & $0,000 \mathrm{E} 0$ & $0,000 \mathrm{E} 0$ & $0,000 \mathrm{E} 0$ & $1,557 \mathrm{E} 6$ \\
\hline P58871 & $182 \mathrm{kDa}$ tankyrase-1-binding protein OS=Mus musculus GN=Tnks1bp1 PE=1 SV=2 - [TB182_MOUSE] & $0,000 \mathrm{E} 0$ & $0,000 \mathrm{E} 0$ & $0,000 \mathrm{E}^{\circ}$ & $8,133 E 6$ \\
\hline Q8R154-2 & Isoform 2 of Metastasis suppressor protein 1 OS=Mus musculus GN=Mtss1 - [MTSS1_MOUSE] & $0,000 \mathrm{E} 0$ & $0,000 \mathrm{E} 0$ & $0,000 \mathrm{E} 0$ & $5,823 \mathrm{E} 6$ \\
\hline Q9D083-3 & Isoform 3 of Kinetochore protein Spc24 OS=Mus musculus GN=Spc24 - [SPC24_MOUSE] & $0,000 \mathrm{E} 0$ & $0,000 E 0$ & $0,000 \mathrm{E}_{0}$ & 8,73455 \\
\hline P17095 & High mobility group protein HMG-I/HMG-Y OS=Mus musculus GN=Hmga1 PE=1 SV=4 - [HMGA1_MOUSE] & $0,000 \mathrm{E} 0$ & $0,000 \mathrm{E} 0$ & $0,000 \mathrm{E} 0$ & $7,761 \mathrm{E} 6$ \\
\hline P70168 & Importin subunit beta-1 $\mathrm{OS}=$ Mus musculus $\mathrm{GN}=\mathrm{Kpnb1}$ PE=1 SV=2 - [IMB1_MOUSE] & $0,000 \mathrm{E} 0$ & $0,000 \mathrm{E} 0$ & $0,000 \mathrm{E} 0$ & $2,376 \mathrm{E} 7$ \\
\hline Q8BWW4 & La-related protein 4 OS=Mus musculus GN=Larp4 PE=1 SV=2 - [LARP4_MOUSE] & $0,000 E_{0}$ & $0,000 \mathrm{E} 0$ & $0,000 E_{0}$ & $2,113 \mathrm{E} 7$ \\
\hline Q8V194 & 2'-5'-oligoadenylate synthase-like protein 1 OS=Mus musculus GN=Oasl1 PE=2 SV=1 - [OASL1_MOUSE] & $0,000 \mathrm{E} 0$ & $0,000 \mathrm{E} 0$ & $0,000 E_{0}$ & $7,767 \mathrm{E}$ \\
\hline Q8BJS4-3 & Isoform 3 of SUN domain-containing protein 2 OS=Mus musculus GN=Sun2 - [SUN2_MOUSE] & $0,000 \mathrm{E} 0$ & $0,000 \mathrm{E} 0$ & $0,000 \mathrm{E} 0$ & $2,530 \mathrm{E} 6$ \\
\hline 070493 & Sorting nexin-12 OS=Mus musculus GN=Snx12 PE=1 SV=1 - [SNX12_MOUSE] & $0,000 E 0$ & $0,000 E 0$ & $0,000 E 0$ & $1,689 E 6$ \\
\hline
\end{tabular}




\begin{tabular}{|c|c|c|c|c|c|}
\hline Q80UM3 & N-alpha-acetyltransferase 15, NatA auxiliary subunit OS=Mus musculus GN=Naa15 PE=1 SV=1 - [NAA15_MOUSE] & $0,000 E_{0}$ & $0,000 E_{0}$ & $0,000 \mathrm{E}_{0}$ & 9,395E6 \\
\hline A2A5Z6 & E3 ubiquitin-protein ligase SMURF2 OS=Mus musculus GN=Smurf2 PE=1 SV=1 - [SMUF2_MOUSE] & $0,000 \mathrm{E} 0$ & $0,000 \mathrm{E} 0$ & $0,000 E 0$ & 9,907E6 \\
\hline Q8K1N4 & Spermatogenesis-associated serine-rich protein 2 OS=Mus musculus GN=Spats2 PE=1 SV=1 - [SPAS2_MOUSE] & $0,000 \mathrm{E} 0$ & $0,000 E 0$ & $0,000 \mathrm{E}^{-}$ & $4,632 E 6$ \\
\hline Q76N33 & AMSH-like protease OS=Mus musculus GN=Stambpl1 PE=1 SV=1 - [STALP_MOUSE] & $0,000 E 0$ & $0,000 E 0$ & $0,000 E 0$ & $4,477 \mathrm{E} 6$ \\
\hline P62196 & 265 protease regulatory subunit 8 OS=Mus musculus GN=Psmc5 PE=1 $\mathrm{SV}=1$ - [PRS8_MOUSE] & $0,000 E 0$ & $0,000 \mathrm{E} 0$ & $0,000 \mathrm{E} 0$ & $6,374 E 5$ \\
\hline P11352 & Glutathione peroxidase $1 \mathrm{OS}=$ Mus musculus $\mathrm{GN}=\mathrm{Gpx} 1 \mathrm{PE}=1 \mathrm{SV}=2$-[GPX1_MOUSE] & $0,000 E_{0}$ & $0,000 E_{0}$ & $0,000 \mathrm{E} 0$ & 1,390E6 \\
\hline Q61753 & D-3-phosphoglycerate dehydrogenase OS=Mus musculus GN=Phgdh PE=1 SV=3 - [SERA_MOUSE] & $0,000 E 0$ & $0,000 E 0$ & $0,000 E 0$ & $1,120 \mathrm{E} 7$ \\
\hline Q9נII7 & AN1-type zinc finger protein 2A OS=Mus musculus GN=Zfand2a PE=2 SV=1 - [ZFN2A_MOUSE] & $0,000 E 0$ & $0,000 E 0$ & $0,000 E_{0}$ & 2,063E6 \\
\hline P97820 & Mitogen-activated protein kinase kinase kinase kinase 4 OS=Mus musculus GN=Map4k4 PE=1 SV=1- [M4K4_MOUSE] & $0,000 \mathrm{EO}$ & $0,000 \mathrm{E} 0$ & 7,335E5 & 1,709E7 \\
\hline P63280 & SUMO-conjugating enzyme UBC9 OS=Mus musculus GN=Ube2i PE=1 SV=1 - [UBC9_MOUSE] & $0,000 E_{0}$ & $0,000 E_{0}$ & $0,000 E 0$ & 3,191E5 \\
\hline Q9נI10-2 & Isoform 2 of Serine/threonine-protein kinase 3 OS=Mus musculus GN=Stk3 - [STK3_MOUSE] & $0,000 E 0$ & $0,000 E 0$ & $0,000 E 0$ & $2,199 \mathrm{E} 6$ \\
\hline Q3UHX2 & 28 kDa heat- and acid-stable phosphoprotein OS=Mus musculus GN=Pdap1 PE=1 SV=1 - [HAP28_MOUSE] & $0,000 E 0$ & $0,000 E 0$ & $0,000 E 0$ & 3,005E7 \\
\hline P61211 & ADP-ribosylation factor-like protein 1 OS=Mus musculus GN=Arl1 PE=1 SV=1 - [ARL1_MOUSE] & $0,000 E_{0}$ & $0,000 E_{0}$ & $0,000 E_{0}$ & $5,809 E 6$ \\
\hline P46061 & Ran GTPase-activating protein 1 OS=Mus musculus GN=Rangap1 PE=1 SV=2 - [RAGP1_MOUSE] & $0,000 E 0$ & $0,000 E_{0}$ & $0,000 E 0$ & $8,310 \mathrm{E} 5$ \\
\hline A2A699-2 & Isoform 2 of Protein FAM171A2 OS=Mus musculus GN=Fam171a2 - [F1712_MOUSE] & $0,000 E 0$ & $0,000 E 0$ & $0,000 E 0$ & 3,642E6 \\
\hline Q3UH60 & Disco-interacting protein 2 homolog B OS=Mus musculus GN=Dip2b PE=1 SV=1 - [DIP2B_MOUSE] & $0,000 E 0$ & $0,000 E 0$ & $0,000 E 0$ & $1,078 \mathrm{E} 7$ \\
\hline Q8VBX6-2 & Isoform 2 of Multiple PDZ domain protein OS=Mus musculus GN=Mpdz - [MPDZ_MOUSE] & $0,000 \mathrm{E}_{0}$ & $0,000 \mathrm{E} 0$ & 0,000E0 & $1,728 \mathrm{E7}$ \\
\hline Q8CFE2 & Histone PARylation factor 1 OS=Mus musculus GN=Hpf1 PE=1 $\mathrm{SV}=1$ - [HPF1_MOUSE] & $0,000 E_{0}$ & $0,000 E_{0}$ & $0,000 E_{0}$ & $2,220 \mathrm{E} 6$ \\
\hline Q8CHC4 & Synaptojanin-1 OS=Mus musculus GN=Synj1 PE=1 SV=3 - [SYNJ1_MOUSE] & $0,000 E 0$ & $0,000 E 0$ & $0,000 E 0$ & 4,993E6 \\
\hline Q6A0A9 & Constitutive coactivator of PPAR-gamma-like protein 1 OS=Mus musculus GN=FAM120A PE=1 SV=2 - [F120A_MOUSE] & $0,000 E 0$ & $0,000 E 0$ & $0,000 E_{0}$ & 5,463E6 \\
\hline 088384 & Vesicle transport through interaction with $\mathrm{t}-\mathrm{SNAREs}$ homolog $1 \mathrm{~B} \mathrm{OS}=$ Mus musculus $\mathrm{GN}=\mathrm{Vti1b} \mathrm{PE}=1 \mathrm{SV}=1$ - [VTI1B_MOUSE] & $0,000 E_{0}$ & $0,000 E_{0}$ & $0,000 \mathrm{E}_{0}$ & $4,794 E 6$ \\
\hline P06869 & Urokinase-type plasminogen activator OS=Mus musculus GN=Plau PE=1 SV=1 - [UROK_MOUSE] & $0,000 E 0$ & $0,000 \mathrm{E} 0$ & $0,000 E 0$ & $5,666 \mathrm{E} 6$ \\
\hline A2AGT1 & Cerebellar degeneration-related protein 2-like OS=Mus musculus GN=Cdr21 PE=1 SV=1 - [CDR2L_MOUSE] & $0,000 E 0$ & $0,000 E 0$ & $0,000 E 0$ & 3,174E6 \\
\hline Q92258 & Kinesin-like protein KIF2C OS=Mus musculus GN=Kif2c PE=1 SV=1 - [KIF2C_MOUSE] & 0,000E0 & 0,000E0 & 0,000E0 & $1,088 \mathrm{E} 7$ \\
\hline Q3UHJO-2 & Isoform 2 of AP2-associated protein kinase 1 OS=Mus musculus GN=Aak1 - [AAK1_MOUSE] & $0,000 E_{0}$ & $0,000 E_{0}$ & $0,000 E_{0}$ & 6,965E6 \\
\hline Q9CQ71 & Replication protein A 14 kDa subunit OS=Mus musculus $\mathrm{GN}=\mathrm{Rpa} 3 \mathrm{PE}=1 \mathrm{SV}=1$ - [RFA3_MOUSE] & $0,000 E_{0}$ & $0,000 E_{0}$ & $0,000 \mathrm{E}_{0}$ & $3,372 E 6$ \\
\hline Q6NXY1 & TBC1 domain family member 31 OS=Mus musculus GN=Tbc1d31 PE=1 SV=1 - [TBC31_MOUSE] & $0,000 \mathrm{E} 0$ & $0,000 \mathrm{E} 0$ & $0,000 E 0$ & $2,719 \mathrm{EE}$ \\
\hline Q921132 & GTP-binding protein Rheb OS=Mus musculus GN=Rheb PE=1 SV=1 - [RHEB_MOUSE] & $0,000 E 0$ & $0,000 E 0$ & $0,000 E 0$ & $1,711 \mathrm{EE}$ \\
\hline Q80296 & Vang-like protein 1 OS=Mus musculus GN=Vangl1 PE=1 SV=2 - [VANG1_MOUSE] & $0,000 E_{0}$ & $0,000 E_{0}$ & $0,000 E_{0}$ & $8,226 E 6$ \\
\hline Q923T9-3 & Isoform 3 of Calcium/calmodulin-dependent protein kinase type II subunit gamma OS=Mus musculus GN=Camk2g - [KCC2G_MOUSE] & $0,000 E 0$ & $0,000 E 0$ & 0,000E0 & 2,093E7 \\
\hline Q920G0 & PDZ domain-containing protein GIPC1 OS=Mus musculus GN=Gipc1 PE=1 SV=1 - [GIPC1_MOUSE] & $0,000 E 0$ & $0,000 E 0$ & $0,000 E 0$ & $2,849 E 6$ \\
\hline Q9JLV1 & BAG family molecular chaperone regulator 3 OS=Mus musculus $\mathrm{GN}=\mathrm{Bag} 3 \mathrm{PE}=1 \mathrm{SV}=2$ - [BAG3_MOUSE] & $0,000 E 0$ & $0,000 E_{0}$ & $0,000 \mathrm{E} 0$ & $2,944 E 6$ \\
\hline 088685 & 265 protease regulatory subunit $6 \mathrm{~A}$ OS $=$ Mus musculus $\mathrm{GN}=\mathrm{Psmc3} \mathrm{PE}=1 \mathrm{SV}=2-$ [PRS6A_MOUSE] & $0,000 E 0$ & $0,000 \mathrm{E} 0$ & $0,000 \mathrm{E} 0$ & $2,886 \mathrm{E} 6$ \\
\hline Q61081 & Hsp90 co-chaperone Cdc37 OS=Mus musculus GN=Cdc37 PE=1 SV=1 - [CDC37_MOUSE] & $0,000 E_{0}$ & $0,000 E_{0}$ & $0,000 E_{0}$ & 9,211E6 \\
\hline Q9DCL9 & Multifunctional protein ADE2 OS=Mus musculus GN=Paics PE=1 SV=4 - [PUR6_MOUSE] & $0,000 E 0$ & $0,000 E 0$ & $0,000 E 0$ & 9,520E6 \\
\hline Q8VDF2-2 & Isoform 2 of E3 ubiquitin-protein ligase UHRF1 OS=Mus musculus GN=Uhrf1 - [UHRF1_MOUSE] & $0,000 E 0$ & $0,000 E 0$ & $0,000 E 0$ & $5,050 \mathrm{E} 6$ \\
\hline Q8C804 & Spindle and centriole-associated protein 1 OS=Mus musculus GN=Spice1 PE=1 SV=2 - [SPICE_MOUSE] & $0,000 E 0$ & $0,000 E 0$ & $0,000 E 0$ & $2,627 \mathrm{E} 6$ \\
\hline Q805Y4 & E3 ubiquitin-protein ligase MIB1 OS=Mus musculus GN=Mib1 PE=1 SV=1 - [MIB1_MOUSE] & $0,000 E 0$ & $0,000 E 0$ & $0,000 E 0$ & 9,291E6 \\
\hline Q9D0BO & Serine/arginine-rich splicing factor $90 \mathrm{OS}=$ Mus musculus GN=Srsf9 PE=1 SV=1 - [SRSF9_MOUSE] & $0,000 E 0$ & $0,000 E 0$ & $0,000 E 0$ & $7,898 \mathrm{E} 6$ \\
\hline Q91YU6 & Leucine zipper putative tumor suppressor 2 OS=Mus musculus GN=LZts2 PE=1 SV=3 - [LZTS2_MOUSE] & $0,000 E_{0}$ & $0,000 E_{0}$ & $0,000 \mathrm{E}_{0}$ & $5,866 \mathrm{E} 6$ \\
\hline Q6P9Q6 & FK506-binding protein 15 OS=Mus musculus GN=Fkbp15 PE=1 SV=2 - [FKB15_MOUSE] & $0,000 E 0$ & $0,000 E 0$ & $0,000 E 0$ & $1,176 \mathrm{E} 7$ \\
\hline P59326 & YTH domain-containing family protein 1 OS=Mus musculus GN=Ythdf1 PE=1 SV=1 - [YTHD1_MOUSE] & $0,000 E 0$ & $0,000 E 0$ & 5,369E6 & $1,748 \mathrm{E} 6$ \\
\hline Q8CJ60 & Protein argonaute-2 OS=Mus musculus GN=Ago2 PE=1 SV=3 - [AGO2_MOUSE] & $0,000 E 0$ & $0,000 E 0$ & $0,000 E 0$ & 9,572E6 \\
\hline Q922J9 & Fatty acyl-CoA reductase 1 OS=Mus musculus GN=Far1 PE=1 SV=1- [FACR1_MOUSE] & $0,000 E 0$ & $0,000 E 0$ & $0,000 E 0$ & $4,808 E 6$ \\
\hline P46471 & 265 protease regulatory subunit 7 OS=Mus musculus GN=Psmc2 PE=1 SV=5 $-[$ PRS7_MOUSE] & $0,000 E 0$ & $0,000 E 0$ & $0,000 E 0$ & $3,186 \mathrm{E} 6$ \\
\hline Q91VJ2 & Protein kinase C delta-binding protein OS=Mus musculus GN=Prkcdbp PE=1 SV=1 - [PRDBP_MOUSE] & $0,000 E 0$ & $0,000 E 0$ & $0,000 E_{0}$ & $1,960 \mathrm{E} 7$ \\
\hline Q9CXW4 & $60 \mathrm{~S}$ ribosomal protein L11 OS=Mus musculus GN=Rpl11 PE=1 SV=4 - [RL11_MOUSE] & 3,381E7 & $0,000 E 0$ & $1,574 E 7$ & $2,628 \mathrm{E} 8$ \\
\hline P70336 & Rho-associated protein kinase $2 \mathrm{OS}=$ Mus musculus $\mathrm{GN}=\mathrm{Rock2} \mathrm{PE}=1 \mathrm{SV}=1$ - [ROCK2_MOUSE] & $0,000 E 0$ & $0,000 E 0$ & $1,352 E 5$ & $8,759 E 6$ \\
\hline P63034-3 & Isoform 3 of Cytohesin-2 OS=Mus musculus GN=Cyth2 - [CYH2_MOUSE] & $0,000 E 0$ & $0,000 E 0$ & $0,000 E 0$ & 6,002E6 \\
\hline P97427 & Dihydropyrimidinase-related protein 1 OS=Mus musculus GN=Crmp1 PE=1 $1 \mathrm{SV}=1$ - [DPYL1_MOUSE] & $0,000 \mathrm{E} 0$ & $0,000 \mathrm{E} 0$ & $0,000 \mathrm{E} 0$ & 3,092E6 \\
\hline Q3UGC7 & Eukaryotic translation initiation factor 3 subunit J-A OS=Mus musculus GN=Eif3j1 PE=2 SV=1 - [EI3]A_MOUSE] & $0,000 E_{0}$ & $0,000 E_{0}$ & $0,000 E_{0}$ & $2,092 E 6$ \\
\hline Q6DFW4 & Nucleolar protein 58 OS=Mus musculus GN=Nop58 PE=1 SV=1 - [NOP58_MOUSE] & $0,000 \mathrm{EO}$ & $0,000 \mathrm{E} 0$ & 0,000E0 & 2,212E6 \\
\hline 070503 & Very-long-chain 3-oxoacyl-CoA reductase OS=Mus musculus GN=Hsd17b12 PE=1 SV=1 - [DHB12_MOUSE] & $0,000 E 0$ & $0,000 E 0$ & $0,000 E 0$ & $6,565 \mathrm{E} 6$ \\
\hline Q9CXY6 & Interleukin enhancer-binding factor 2 OS=Mus musculus $\mathrm{GN}=\mathrm{IfF} 2 \mathrm{PE}=1 \mathrm{SV}=1$ - [LLF2_MOUSE] & $0,000 E 0$ & $0,000 E_{0}$ & $0,000 E 0$ & $2,362 E 6$ \\
\hline Q8K227-2 & Isoform 2 of RELT-like protein 1 OS=Mus musculus GN=Rell1 - [RELL1_MOUSE] & $0,000 E_{0}$ & $0,000 E_{0}$ & $0,000 \mathrm{E}_{0}$ & $2,517 \mathrm{E} 6$ \\
\hline Q9DD03 & Ras-related protein Rab-13 OS=Mus musculus GN=Rab13 PE=1 SV=1 - [RAB13_MOUSE] & $0,000 E_{0}$ & $0,000 E 0$ & $0,000 \mathrm{E} 0$ & 3,615E7 \\
\hline Q3UFY8 & Mitochondrial ribonuclease P protein 1 OS=Mus musculus GN=Trmt10c PE=1 SV=2 - [MRRP1_MOUSE] & $0,000 E_{0}$ & $0,000 E_{0}$ & $0,000 E_{0}$ & $2,560 \mathrm{E} 6$ \\
\hline 070311 & Glycylpeptide $\mathrm{N}$-tetradecanoyytransferase 2 OS=Mus musculus $\mathrm{GN}=\mathrm{Nmt2} P \mathrm{PE}=1 \mathrm{SV}=1$ - [NMT2_MOUSE] & $0,000 E 0$ & $0,000 E 0$ & $0,000 E_{0}$ & $5,967 \mathrm{E} 6$ \\
\hline P11276 & Fibronectin OS=Mus musculus GN=Fn1 PE=1 SV=4 - [FINC_MOUSE] & $0,000 E 0$ & $0,000 E 0$ & $0,000 E 0$ & $1,421 E 7$ \\
\hline Q07832 & Serine/threonine-protein kinase PLK1 OS=Mus musculus GN=PIK1 PE=1 SV=2 - [PLK1_MOUSE] & $0,000 E 0$ & $0,000 E 0$ & $0,000 \mathrm{E} 0$ & 3,303E6 \\
\hline P63038 & $60 \mathrm{kDa}$ heat shock protein, mitochondrial OS=Mus musculus GN=Hspd1 PE=1 SV=1-[CH60_MOUSE] & $0,000 \mathrm{E} 0$ & $0,000 \mathrm{E} 0$ & 2,327E6 & $1,869 \mathrm{E} 6$ \\
\hline Q88ZX3-3 & Isoform 3 of POC1 centriolar protein homolog A OS=Mus musculus GN=Poc1a - [POC1A_MOUSE] & $0,000 E_{0}$ & $0,000 E_{0}$ & $0,000 \mathrm{E}_{0}$ & 1,943E6 \\
\hline P00493 & Hypoxanthine-guanine phosphoribosyltransferase OS=Mus musculus GN=Hprt1 PE=1 SV=3 - [HPRT_MOUSE] & $0,000 E 0$ & $0,000 E 0$ & $0,000 E 0$ & $1,010 \mathrm{E} 6$ \\
\hline Q9DBU6 & Serine/Arginine-related protein 53 OS=Mus musculus GN=Rsrc1 PE=1 SV=1 - [RSRC1_MOUSE] & $0,000 E 0$ & $0,000 E 0$ & $0,000 E 0$ & 7,670E6 \\
\hline QOVGY8 & Protein TANC1 OS=Mus musculus GN=Tanc1 PE=1 SV=2 - [TANC1_MOUSE] & $0,000 E_{0}$ & $0,000 E 0$ & $0,000 \mathrm{E}_{0}$ & $4,532 E 6$ \\
\hline 055029 & Coatomer subunit beta' OS=Mus musculus $\mathrm{GN}=\mathrm{COpb} 2 \mathrm{PE}=1 \mathrm{SV}=2$ - [COPB2_MOUSE] & $0,000 E 0$ & $0,000 E_{0}$ & $0,000 \mathrm{E} 0$ & 3,313E6 \\
\hline Q9EPK2-3 & Isoform 3 of Protein XRP2 OS=Mus musculus GN=RP2 - [XRP2_MOUSE] & $0,000 E_{0}$ & $0,000 E_{0}$ & $0,000 \mathrm{E}_{0}$ & $6,839 \mathrm{E} 6$ \\
\hline AZAIIO & MAP7 domain-containing protein 1 OS=Mus musculus GN=Map7d1 PE=1 SV=1 - [MA7D1_MOUSE] & $0,000 E_{0}$ & $0,000 E_{0}$ & $0,000 \mathrm{E} 0$ & $2,432 \mathrm{EE}$ \\
\hline B2RY56 & RNA-binding protein 25 OS=Mus musculus GN=Rbm25 PE=1 SV=2 - [RBM25_MOUSE] & $0,000 E_{0}$ & $0,000 E_{0}$ & $0,000 E_{0}$ & 7,150E6 \\
\hline Q9נJK2 & LanC-like protein 2 OS=Mus musculus GN=Lanc12 PE=1 SV=1 - [LANC2_MOUSE] & $0,000 E 0$ & $0,000 E 0$ & $0,000 E_{0}$ & $2,190 \mathrm{E} 7$ \\
\hline P14602-2 & Isoform B of Heat shock protein beta-1 OS=Mus musculus GN=Hspb1 - [HSPB1_MOUSE] & $0,000 E 0$ & $0,000 E 0$ & $6,467 \mathrm{E} 6$ & 7,573E6 \\
\hline Q3TW96 & UDP-N-acetylhexosamine pyrophosphorylase-like protein 1 OS=Mus musculus GN=Uap111 PE=1 SV=1 - [UAP1L_MOUSE] & $0,000 E 0$ & $0,000 E 0$ & 0,000E0 & $3,531 \mathrm{E} 6$ \\
\hline Q3UIA2 & Rho GTPase-activating protein 17 OS=Mus musculus GN=Arhgap17 PE=1 SV=1- [RHG17_MOUSE] & $0,000 E 0$ & $0,000 E 0$ & $0,000 E 0$ & $2,402 E 6$ \\
\hline Q999N2 & Kelch-like protein 22 OS=Mus musculus GN=KIIII2 PE=1 SV=1 - [KLH22_MOUSE] & $0,000 E_{0}$ & $0,000 E_{0}$ & $0,000 E_{0}$ & $3,111 \mathrm{EE}$ \\
\hline Q8BMA6 & Signal recognition particle subunit SRP68 OS=Mus musculus GN=Srp68 PE=1 SV=2 - [SRP68_MOUSE] & $0,000 E 0$ & $0,000 E 0$ & $0,000 E 0$ & $4,619 \mathrm{E} 6$ \\
\hline P30285 & Cyclin-dependent kinase 4 OS=Mus musculus GN=Cdk4 PE=1 SV=1- [CDK4_MOUSE] & $0,000 E_{0}$ & $0,000 E_{0}$ & $0,000 E_{0}$ & 9,391E6 \\
\hline Q91YD3 & mRNA-decapping enzyme $1 \mathrm{~A}$ OS=Mus musculus GN=DCp1a PE=1 SV=1 - [DCP1A_MOUSE] & $0,000 E_{0}$ & $0,000 E_{0}$ & $0,000 \mathrm{E}_{0}$ & $3,234 E 6$ \\
\hline Q5SXY1-3 & Isoform 4 of Cytospin-B OS=Mus musculus GN=Specc1 - [CYTSB_MOUSE] & $0,000 E_{0}$ & $0,000 E_{0}$ & $0,000 \mathrm{E}_{0}$ & $3,509 E 6$ \\
\hline Q9ET54-4 & Isoform 4 of Palladin OS=Mus musculus GN=Palld - [PALLD_MOUSE] & $0,000 E 0$ & $0,000 E 0$ & $0,000 \mathrm{E} 0$ & $1,035 E 7$ \\
\hline P61963 & DDB1- and CUL4-associated factor 7 OS=Mus musculus GN=Dcaf7 PE $=1 \mathrm{SV}=1$ - [DCAF7_MOUSE] & $0,000 E_{0}$ & $0,000 E_{0}$ & $0,000 E_{0}$ & $2,564 E 6$ \\
\hline Q8VEH3 & ADP-ribosylation factor-like protein $8 \mathrm{~A}$ OS=Mus musculus GN=Arl8a PE=1 SV=1 - [ARL8A_MOUSE] & $0,000 E_{0}$ & $0,000 E_{0}$ & $0,000 \mathrm{E} 0$ & 3,034E6 \\
\hline P61759 & Prefoldin subunit 3 OS=Mus musculus GN=Vbp1 PE=1 SV=2 - [PFD3_MOUSE] & $0,000 E 0$ & $0,000 E_{0}$ & $0,000 E 0$ & 1,069E6 \\
\hline Q9JMK2 & Casein kinase I isoform epsilon OS=Mus musculus GN=Csnk1e PE=1 SV=2 - [KC1E_MOUSE] & $0,000 E 0$ & $0,000 E_{0}$ & $0,000 \mathrm{E} 0$ & $1,802 E 7$ \\
\hline Q677W8 & PERQ amino acid-rich with GYF domain-containing protein 2 OS=Mus musculus GN=Gigyf2 PE=1 SV=2 - [PERQ2_MOUSE] & $0,000 E_{0}$ & $0,000 \mathrm{E} 0$ & $0,000 \mathrm{E}_{0}$ & $6,806 \mathrm{E} 6$ \\
\hline Q62318 & Transcription intermediary factor 1-beta OS=Mus musculus GN=Trim28 PE=1 SV=3 - [TIF1B_MOUSE] & $0,000 E 0$ & $0,000 E 0$ & $0,000 \mathrm{E} 0$ & $4,817 \mathrm{E} 6$ \\
\hline P21981 & Protein-glutamine gamma-glutamyltransferase 2 OS=Mus musculus GN=Tgm2 PE=1 SV=4 - [TGM2_MOUSE] & $0,000 E 0$ & $0,000 E 0$ & $0,000 E_{0}$ & $4,802 E 6$ \\
\hline Q9ESX5 & H/ACA ribonucleoprotein complex subunit 4 OS=Mus musculus GN=Dkc1 PE=1 SV=4 - [DKC1_MOUSE] & $0,000 E 0$ & $0,000 E 0$ & $0,000 \mathrm{E} 0$ & 3,347E6 \\
\hline Q9CZ44 & NSFL1 cofactor p47 OS=Mus musculus GN=Nsfl1c PE=1 SV=1 - [NSF1C_MOUSE] & $0,000 E_{0}$ & $0,000 E_{0}$ & $0,000 \mathrm{E}_{0}$ & $1,622 E 6$ \\
\hline P47941 & Crk-like protein OS=Mus musculus GN=Crkl PE=1 SV=2 - [CRKL_MOUSE] & $0,000 E 0$ & $0,000 E 0$ & $0,000 \mathrm{E} 0$ & $1,295 \mathrm{E} 6$ \\
\hline P81270-3 & Isoform 1 of Transcriptional regulator ERG OS=Mus musculus GN=Erg - [ERG_MOUSE] & $0,000 \mathrm{E} 0$ & $0,000 \mathrm{E} 0$ & $0,000 \mathrm{E}_{0}$ & $1,590 \mathrm{E} 7$ \\
\hline Q91VX2 & Ubiquitin-associated protein 2 OS=Mus musculus GN=Ubap2 PE=1 SV=1 - [UBAP2_MOUSE] & $0,000 E 0$ & $0,000 E 0$ & $0,000 E 0$ & $2,538 \mathrm{E7}$ \\
\hline P56959 & RNA-binding protein FUS OS=Mus musculus GN=Fus PE=1 SV=1 - [FUS_MOUSE] & $0,000 E_{0}$ & $0,000 E_{0}$ & $0,000 E_{0}$ & 1,050E8 \\
\hline Q8C570 & mRNA export factor OS=Mus musculus GN=Rae1 PE=1 SV=1 - [RAE1L_MOUSE] & $0,000 \mathrm{EO}$ & $0,000 E 0$ & $0,000 E 0$ & $4,069 E 6$ \\
\hline
\end{tabular}




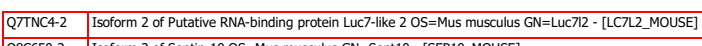

\begin{tabular}{|c|c|}
\hline QTTNC4-2 & Isoform 2 of Putative RNA-binding protein Luc7-like 2 OS=Mus musculus GN=Luc712 - [LC7L2_MOUSE] \\
\hline Q8C650-2 & Isoform 2 of Septin-10 OS=Mus musculus GN=Sept10 - [SEP10_MOUSE] \\
\hline 070479 & BTB/POZ domain-containing adapter for CUL3-mediated RhoA degradation protein 2 OS=Mus musculus GN=Tnfaip1 PE=2 SV=1 - [BACD2_MOUSE] \\
\hline P97376 & Protein FRG1 OS=Mus musculus GN=Frg1 PE=1 SV=2 - [FRG1_MOUSE] \\
\hline Q8BH59 & Calcium-binding mitochondrial carrier protein Aralar1 OS=Mus musculus GN=Slc25a12 PE=1 SV=1 - [CMC1_MOUSE] \\
\hline Q80VP1 & Epsin-1 OS=Mus musculus GN=Epn1 PE=1 SV=3 - [EPN1_MOUSE] \\
\hline Q60770 & Syntaxin-binding protein 3 OS=Mus musculus GN=Stxbp3 PE=1 SV=1 - [STXB3_MOUSE] \\
\hline Q9DBF7 & Pre-mRNA-splicing factor CWC25 homolog OS=Mus musculus GN=CwC25 PE=2 SV=2 - [CWC25_MOUSE] \\
\hline Q6P939 & Anoctamin-6 OS=Mus musculus GN=Ano6 PE=1 SV=1 - [ANO6_MOUSE] \\
\hline P55012 & Solute carrier family 12 member 2 OS=Mus musculus GN=Slc12a2 PE=1 SV=2 - [S12A2_MOUSE] \\
\hline Q69ZH9 & Rho GTPase-activating protein 23 OS=Mus musculus GN=Arhgap23 PE=1 SV=2 - [RHG23_MOUSE] \\
\hline 088983 & Syntaxin-8 OS=Mus musculus GN=SŁ×8 PE=1 SV=1 - [STX8_MOUSE] \\
\hline P19096 & Fatty acid synthase $\mathrm{OS}=$ Mus musculus $\mathrm{GN}=\mathrm{Fasn} \mathrm{PE}=1 \mathrm{SV}=2-$ [FAS_MOUSE] \\
\hline Q61595-3 & Isoform 3 of Kinectin OS=Mus musculus GN=Ktn1 - [KTN1_MOUSE] \\
\hline Q61696 & Heat shock $70 \mathrm{kDa}$ protein $1 \mathrm{~A}$ OS=Mus musculus GN=Hspa1a PE=1 SV=2 - [HS71A_MOUSE] \\
\hline Q9W660 & Glycogen synthase kinase-3 beta OS=Mus musculus GN=Gsk3b PE=1 SV=2 - [GSK3B_MOUSE] \\
\hline Q9EQH3 & Vacuolar protein sorting-associated protein 35 OS=Mus musculus GN=Vps35 PE=1 SV=1 - [VPS35_MOUSE] \\
\hline Q8BP47 & Asparagine--tRNA ligase, cytoplasmic OS=Mus musculus GN=Nars PE=1 SV=2 - [SYNC_MOUSE] \\
\hline Q80072 & Protein scribble homolog OS=Mus musculus GN=Scrib PE=1 SV=2 - [SCRIB_MOUSE] \\
\hline 008807 & Peroxiredoxin-4 OS=Mus musculus GN=Prdx4 PE=1 SV=1 - [PRDX4_MOUSE] \\
\hline Q3UMCO & Spermatogenesis-associated protein 5 OS=Mus musculus GN=Spata5 PE=1 SV=2 - [SPAT5_MOUSE] \\
\hline $008638-2$ & Isoform 2 of Myosin-11 OS=Mus musculus GN=Myh11 - [MYH11_MOUSE] \\
\hline Q9CQ62 & 2,4-dienoyl-COA reductase, mitochondrial OS=Mus musculus GN=Decr1 PE=1 SV=1 - [DECR_MOUSE] \\
\hline P97473 & RISC-loading complex subunit TARBP2 OS=Mus musculus GN=Tarbp2 PE=1 SV=2 - [TRBP2_MOUSE] \\
\hline Q78HU3 & Multivesicular body subunit 12A OS=Mus musculus GN=Mvb12a PE=1 SV=1 - [MB12A_MOUSE] \\
\hline Q9Z110-2 & Isoform Short of Delta-1-pyrroline-5-carboxylate synthase OS=Mus musculus GN=Aldh18a1 - [P5CS_MOUSE] \\
\hline Q62093 & Serine/arginine-rich splicing factor $20 \mathrm{OS}=$ Mus musculus $\mathrm{GN}=\mathrm{Sr} r \mathrm{f} 2 \mathrm{PE}=1 \mathrm{SV}=4$ - [SRSF2_MOUSE] \\
\hline Q99JB2 & Stomatin-like protein 2, mitochondrial OS=Mus musculus GN=Stoml2 PE=1 SV=1 - [STML2_MOUSE] \\
\hline Q99MI1 & ELKS/Rab6-interacting/CAST family member 1 OS=Mus musculus GN=Erc1 PE=1 SV=1 - [RBGI2_MOUSE] \\
\hline P62334 & 265 protease regulatory subunit 10B OS=Mus musculus GN=Psmc6 PE=1 SV=1 - [PRS10_MOUSE] \\
\hline Q3UJV1 & Coiled-coil domain-containing protein 61 OS=Mus musculus GN=Ccdc61 PE=1 SV=1- [CCD61_MOUSE] \\
\hline P28798 & Granulins OS=Mus musculus GN=Grn PE=1 SV=2 - [GRN_MOUSE] \\
\hline 008573-3 & Isoform 3 of Galectin-9 OS=Mus musculus GN=Lgals9 - [LEG9_MOUSE] \\
\hline Q6PDN3-2 & Isoform 2 of Myosin light chain kinase, smooth muscle OS=Mus musculus GN=Mylk - [MYLK_MOUSE] \\
\hline P11928 & 2'-5'-oligoadenylate synthase $1 \mathrm{~A}$ OS=Mus musculus $\mathrm{GN}=$ Oas1a PE $=1 \mathrm{SV}=2$ - [OAS1A_MOUSE] \\
\hline P45376 & Aldose reductase $\mathrm{OS}=$ Mus musculus GN=Akr1b1 PE=1 SV=3 - [ALDR_MOUSE] \\
\hline P62892 & 60S ribosomal protein L39 OS=Mus musculus GN=Rpl39 PE=1 SV=2 - [RL39_MOUSE] \\
\hline P18654 & Ribosomal protein S6 kinase alpha-3 OS=Mus musculus GN=Rps6ka3 PE=1 SV=2 - [KS6A3_MOUSE] \\
\hline Q9D0M3-2 & Isoform 2 of Cytochrome c1, heme protein, mitochondrial OS=Mus musculus GN=Cyc1 - [CY1_MOUSE] \\
\hline
\end{tabular}

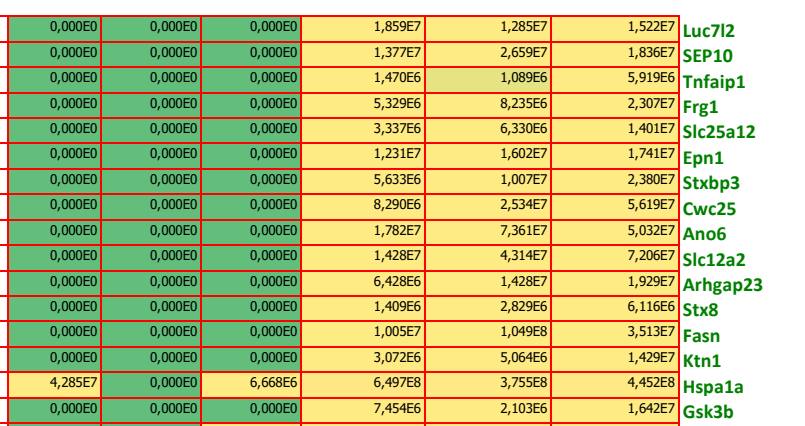




\begin{tabular}{|c|c|c|c|c|c|}
\hline Q80TH2-1 & Isoform 1 of Erbin OS=Mus musculus GN=Erbin - [ERBIN_MOUSE] & $0,000 E_{0}$ & $0,000 E 0$ & $0,000 \mathrm{E}_{0}$ & $2,347 E 6$ \\
\hline Q8VBT6 & Apolipoprotein B receptor OS=Mus musculus GN=Apobr PE=1 SV=1 - [APOBR_MOUSE] & $0,000 E_{0}$ & $0,000 E 0$ & $0,000 \mathrm{E}^{-}$ & $3,354 E 6$ \\
\hline Q9CW39 & Bifunctional purine biosynthesis protein PURH OS=Mus musculus GN=Atic PE=1 SV=2 - [PUR9_MOUSE] & $0,000 \mathrm{E} 0$ & $0,000 E 0$ & $0,000 \mathrm{E}^{-}$ & $2,409 E 6$ \\
\hline A2AQ19 & RNA polymerase-associated protein RTF1 homolog OS=Mus musculus GN=Rtf1 PE=1 SV=1 - [RTF1_MOUSE] & $0,000 E 0$ & $0,0000_{0}$ & $0,000 E 0$ & $5,034 E 6$ \\
\hline P39688 & Tyrosine-protein kinase Fyn OS=Mus musculus GN=Fyn PE=1 SV=4 - [FYN_MOUSE] & $0,000 E_{0}$ & $0,000 E 0$ & $2,114 E 5$ & 1,442E7 \\
\hline Q9CR62 & Mitochondrial 2-oxoglutarate/malate carrier protein OS=Mus musculus GN=SIc25a11 PE=1 SV=3 - [M2OM_MOUSE] & $0,000 E_{0}$ & $0,000 E 0$ & $0,000 \mathrm{E} 0$ & 6,059E6 \\
\hline P63005 & Platelet-activating factor acetylhydrolase IB subunit alpha OS=Mus musculus GN=Pafah1b1 PE=1 SV=2 - [LS1_MOUSE] & $0,000 E 0$ & $0,000 E 0$ & $0,000 E 0$ & $8,818 E 6$ \\
\hline Q972X8 & Kelch-like ECH-associated protein 1 OS=Mus musculus GN=Keap1 PE=1 SV=1 - [KEAP1_MOUSE] & $0,000 E 0$ & $0,000 E 0$ & $0,000 E_{0}$ & $2,604 E 6$ \\
\hline P97386-2 & Isoform Beta of DNA ligase 3 OS=Mus musculus GN=Lig3 - [DNLI3_MOUSE] & $0,000 E 0$ & $0,000 E 0$ & $0,000 E 0$ & $3,950 \mathrm{E} 6$ \\
\hline Q8VHK9 & ATP-dependent RNA helicase DHX36 OS=Mus musculus GN=Dhx36 PE=1 SV=2 - [DHX36_MOUSE] & $0,000 E_{0}$ & $0,000 E 0$ & $0,000 E 0$ & 6,937E6 \\
\hline Q61846 & Maternal embryonic leucine zipper kinase OS=Mus musculus GN=Melk PE=1 SV=2 - [MELK_MOUSE] & $0,000 E 0$ & $0,000 E 0$ & $0,000 E 0$ & 1,905E6 \\
\hline Q8CHH9 & Septin-8 OS=Mus musculus GN=Sept8 PE=1 SV=4 - [SEPT8_MOUSE] & $0,000 E 0$ & $0,000 E_{0}$ & $0,000 E 0$ & $1,802 E 7$ \\
\hline Q80UP5 & Ankyrin repeat domain-containing protein 13A OS=Mus musculus GN=Ankrd13a PE=1 SV=2 - [AN13A_MOUSE] & $0,000 E 0$ & $0,000 E 0$ & $0,000 E 0$ & $2,476 \mathrm{E} 6$ \\
\hline Q88570 & Synaptosomal-associated protein 47 OS=Mus musculus GN=Snap47 PE=1 SV=1 - [SNP47_MOUSE] & $0,000 E 0$ & $0,000 E 0$ & $0,000 E 0$ & 9,854E5 \\
\hline P50516 & V-type proton ATPase catalytic subunit A OS=Mus musculus GN=Atp6v1a PE=1 SV=2 - [VATA_MOUSE] & $0,000 E 0$ & $0,000 E 0$ & $0,000 E 0$ & 2,553E6 \\
\hline Q69ZB0-3 & Isoform 3 of Leucine-rich repeat and coiled-coil domain-containing protein 1 OS=Mus musculus GN=Lrrcc1 - [LRCC1_MOUSE] & $0,000 E 0$ & $0,000 E 0$ & $0,000 E_{0}$ & $2,347 E 6$ \\
\hline Q99KK2 & N-acylneuraminate cytidyly|transferase OS=Mus musculus GN=Cmas PE=1 SV=2 - [NEUA_MOUSE] & $0,000 \mathrm{E}_{0}$ & $0,000 E 0$ & $0,000 \mathrm{E} 0$ & $1,756 \mathrm{E} 6$ \\
\hline Q62172 & RalA-binding protein 1 OS=Mus musculus GN=Ralbp1 PE=1 SV=4 - [RBP1_MOUSE] & $0,000 E_{0}$ & $0,000 E 0$ & $0,000 E_{0}$ & $2,565 \mathrm{E} 7$ \\
\hline BRRQC6 & CAD protein OS=Mus musculus GN=Cad PE=1 SV=1 - [PYR1_MOUSE] & $0,000 E 0$ & $0,000 E 0$ & $0,000 E 0$ & 5,483E6 \\
\hline Q1HFZO & tRNA (cytosine(34)-C(5))-methyltransferase OS=Mus musculus GN=Nsun2 PE=1 SV=2 - [NSUN2_MOUSE] & $0,000 E 0$ & $0,000 E 0$ & $0,000 E_{0}$ & $2,205 E 6$ \\
\hline Q68FHO & Plakophilin-4 OS=Mus musculus GN=Pkp4 PE=1 SV=1 - [PKP4_MOUSE] & $0,000 E_{0}$ & $0,000 E 0$ & $0,000 E_{0}$ & $2,776 \mathrm{E} 6$ \\
\hline Q3TGF2 & Protein FAM107B OS=Mus musculus GN=Fam107b PE=1 SV=2 - [F107B_MOUSE] & $0,000 E 0$ & $0,000 E 0$ & $0,000 E 0$ & $2,030 \mathrm{E} 6$ \\
\hline Q8BMB3 & Eukaryotic translation initiation factor $4 \mathrm{E}$ type $2 \mathrm{OS}=$ Mus musculus GN=Eif4e2 PE=1 SV=1 - [IF4E2_MOUSE] & $0,000 E 0$ & $0,000 E 0$ & $0,000 \mathrm{E} 0$ & $8,189 \mathrm{E} 6$ \\
\hline Q80XI4 & Phosphatidylinositol 5-phosphate 4-kinase type-2 beta OS=Mus musculus GN=Pip4k2b PE=1 SV=1 - [PI42B_MOUSE] & 0,000E0 & $0,000 E 0$ & $0,000 E_{0}$ & 2,082E6 \\
\hline Q9D8W5 & $26 \mathrm{~S}$ proteasome non-ATPase regulatory subunit $12 \mathrm{OS}=$ Mus musculus GN=Psmd12 PE=1SV=4 - [PSD12_MOUSE] & $0,000 E_{0}$ & $0,000 E 0$ & $0,000 E_{0}$ & 1,880E6 \\
\hline Q8R2Q8 & Bone marrow stromal antigen $2 \mathrm{OS}=$ Mus musculus $\mathrm{GN}=\mathrm{Bst2} 2 \mathrm{PE}=1 \mathrm{SV}=1$ - [BST2_MOUSE] & $0,000 E_{0}$ & $0,000 E 0$ & $0,000 \mathrm{E}_{0}$ & $4,800 \mathrm{E} 7$ \\
\hline Q923D4 & Splicing factor $3 \mathrm{~B}$ subunit $5 \mathrm{OS}=$ Mus musculus $\mathrm{GN}=\mathrm{S} F \mathrm{~b} b 5 \mathrm{PE}=1 \mathrm{SV}=1$ - [SF3B5_MOUSE] & $0,000 \mathrm{E} 0$ & $0,000 E 0$ & $0,000 E 0$ & 3,545E6 \\
\hline P97465 & Docking protein 1 OS=Mus musculus GN=Dok1 PE=1 SV=2 - [DOK1_MOUSE] & $0,000 E_{0}$ & $0,000 E 0$ & $0,000 \mathrm{E} 0$ & 3,292E6 \\
\hline P28656 & Nucleosome assembly protein 1 -like 1 OS=Mus musculus GN=Nap111 PE=1 SV=2 - [NP1L1_MOUSE] & $0,000 E_{0}$ & $0,000 E 0$ & $0,000 E_{0}$ & $8,986 E 6$ \\
\hline Q9QX11-2 & Isoform 2 of Cytohesin-1 OS=Mus musculus GN=Cyth1 - [CYH1_MOUSE] & $0,000 E_{0}$ & $0,000 E 0$ & $0,000 E 0$ & $4,744 E 6$ \\
\hline Q64511 & DNA topoisomerase 2-beta OS=Mus musculus GN=Top2b PE=1 SV=2 - [TOP2B_MOUSE] & $0,000 E 0$ & $0,000 E 0$ & 0,000E0 & $1,330 \mathrm{E} 7$ \\
\hline P61164 & Alpha-centractin OS=Mus musculus GN=Actr1a PE=1 SV=1 - [ACTZ_MOUSE] & $0,000 E 0$ & $0,000 E 0$ & $0,000 \mathrm{E} 0$ & $5,285 E 6$ \\
\hline P17257 & Protein FAM167B OS=Mus musculus GN=Fam167b PE=2 SV=2 - [F167B_MOUSE] & $0,000 E 0$ & $0,000 E 0$ & $0,000 \mathrm{E} 0$ & $5,233 \mathrm{E} 6$ \\
\hline Q9EST5 & Acidic leucine-rich nuclear phosphoprotein 32 family member B OS=Mus musculus GN=Anp32b PE=1SV=1 - [AN32B_MOUSE] & $0,000 E_{0}$ & $0,000 E 0$ & $0,000 E_{0}$ & $3,187 \mathrm{E} 6$ \\
\hline P35235 & Tyrosine-protein phosphatase non-receptor type 11 OS=Mus musculus GN=Ptpn11 PE=1 SV=2 - [PTN11_MOUSE] & $0,000 E 0$ & $0,000 E 0$ & $0,000 E 0$ & 2,902E6 \\
\hline Q781K2 & Up-regulated during skeletal muscle growth protein 5 OS=Mus musculus GN=Usmg5 PE=1 $\mathrm{SV}=1$ - [USMG5_MOUSE] & $0,000 E_{0}$ & $0,000 E 0$ & $0,000 E 0$ & $3,272 E 6$ \\
\hline Q8VEM8 & Phosphate carrier protein, mitochondrial OS=Mus musculus GN=SIc25a3 PE=1 SV=1 - [MPCP_MOUSE] & $0,000 E 0$ & $0,000 E 0$ & 0,000E0 & 2,277E7 \\
\hline Q9RONO & Galactokinase OS=Mus musculus GN=Galk1 PE=1 SV=2 - [GALK1_MOUSE] & $0,000 E 0$ & $0,000 E_{0}$ & $0,000 E 0$ & $8,671 \mathrm{EE}$ \\
\hline Q99NH2-3 & Isoform 3 of Partitioning defective 3 homolog OS=Mus musculus GN=Pard3 - [PARD3_MOUSE] & $0,000 E 0$ & $0,000 E 0$ & $0,000 E 0$ & $3,627 \mathrm{E} 6$ \\
\hline Q6RHR9 & Membrane-associated guanylate kinase, WW and PDZ domain-containing protein 1 OS=Mus musculus GN=Magi1 PE=1 SV=1 - [MAGI1_MOUSE] & $0,000 E_{0}$ & $0,000 E 0$ & $0,0000_{0}$ & 9,352E6 \\
\hline Q9JLV5 & Cullin-3 OS=Mus musculus GN=Cul3 PE=1 SV=1 - [CUL3_MOUSE] & $0,000 E 0$ & $0,000 E 0$ & 0,000E0 & $5,636 \mathrm{EE}$ \\
\hline Q64324 & Syntaxin-binding protein 2 OS=Mus musculus GN=Stxbp2 PE=1 $1 \mathrm{SV}=1$ - [STXB2_MOUSE] & $0,000 E_{0}$ & $0,000 E 0$ & $0,000 E_{0}$ & $4,569 E 5$ \\
\hline Q9Q288 & Vacuolar protein sorting-associated protein 29 OS=Mus musculus GN=VPs29 PE=1 SV=1 - [VPS29_MOUSE] & $0,000 E 0$ & $0,000 E 0$ & $0,000 E 0$ & 4,303E6 \\
\hline Q9CYH6 & Ribosome biogenesis regulatory protein homolog OS=Mus musculus GN=Rrs1 PE=1 SV=1 - [RRS1_MOUSE] & $0,000 E 0$ & $0,000 E 0$ & $0,000 E 0$ & $7,456 \mathrm{E}$ \\
\hline Q9WV02-2 & Isoform 2 of RNA-binding motif protein, $X$ chromosome OS=Mus musculus GN=RbmX - [RBMX_MOUSE] & $0,000 E 0$ & $0,000 E 0$ & $0,000 E 0$ & $4,958 \mathrm{E} 6$ \\
\hline Q924C6 & Lysyl oxidase homolog 4 OS=Mus musculus GN=Loxl4 PE=2 SV=2 - [LOXL4_MOUSE] & $0,000 E 0$ & $0,000 E 0$ & 0,000E0 & 7,417E6 \\
\hline P15379-2 & Isoform 13 of $\mathrm{CD} 44$ antigen OS=Mus musculus $\mathrm{GN}=\mathrm{Cd} 44$ - [CD44_MOUSE] & $0,000 E 0$ & $0,000 E 0$ & $0,000 E 0$ & $3,270 \mathrm{E} 6$ \\
\hline Q9CQV6 & Microtubule-associated proteins 1A/1B light chain 3B OS=Mus musculus GN=Map11/3b PE=1 SV=3 - [MLP3B_MOUSE] & $0,000 E 0$ & $0,000 E 0$ & $0,000 E_{0}$ & 9,970E6 \\
\hline Q9CZH8-2 & Isoform 2 of Coiled-coil domain-containing protein 77 OS=Mus musculus GN=Ccdc77 - [CCD77_MOUSE] & $0,000 E 0$ & $0,000 E 0$ & $0,000 E 0$ & $2,014 E 6$ \\
\hline Q91WB7 & Ubiquitin domain-containing protein 1 OS=Mus musculus GN=Ubtd1 PE=2 SV=1 - [UBTD1_MOUSE] & $0,000 E_{0}$ & $0,000 E 0$ & $0,000 \mathrm{E}_{0}$ & 1,787E6 \\
\hline Q99NB9 & Splicing factor 3B subunit $1 \mathrm{OS}=$ Mus musculus $\mathrm{GN}=\mathrm{S} F 3 \mathrm{~B} 1 \mathrm{PE}=1 \mathrm{SV}=1-$ [SF3B1_MOUSE] & $0,000 E_{0}$ & $0,000 E 0$ & $0,000 E_{0}$ & $2,034 E 6$ \\
\hline Q60848-2 & Isoform 2 of Lymphocyte-specific helicase OS=Mus musculus GN=Hells - [HELLS_MOUSE] & $0,000 E_{0}$ & $0,000 E 0$ & $0,000 \mathrm{E}^{-}$ & $2,855 E 6$ \\
\hline 054946 & Dna] homolog subfamily B member 6 OS=Mus musculus GN=Dnajb6 PE=1 $1 \mathrm{SV}=4$ - [DNJB6_MOUSE] & $0,000 E 0$ & $0,000 E 0$ & 0,000E0 & 9,339E6 \\
\hline Q8K2×3 & CST complex subunit STN1 OS=Mus musculus GN=Stn1 PE=1 SV=2 - [STN1_MOUSE] & $0,000 E 0$ & $0,000 E 0$ & $0,000 E 0$ & 4,694E6 \\
\hline A2RSY6 & TRMT1-like protein OS=Mus musculus GN=Trmt11 PE=1 SV=1 - [TRM1L_MOUSE] & $0,000 \mathrm{E} 0$ & $0,000 E 0$ & $0,000 \mathrm{E} 0$ & $5,755 \mathrm{E} 6$ \\
\hline Q3TKT4 & Transcription activator BRG1 OS=Mus musculus GN=Smarca4 PE=1 SV=1 - [SMCA4_MOUSE] & $0,000 E_{0}$ & $0,000 E 0$ & $0,000 \mathrm{E} 0$ & $5,388 E 6$ \\
\hline P97432 & Next to BRCA1 gene 1 protein OS=Mus musculus GN=Nbr1 PE=1 $1 \mathrm{SV}=1$ - [NBR1_MOUSE] & $0,000 \mathrm{E} 0$ & $0,0000_{0}$ & 0,000E0 & $1,301 \mathrm{E7}$ \\
\hline Q91V92 & ATP-citrate synthase $0 \mathrm{~S}=$ Mus musculus $\mathrm{GN}=\mathrm{Acly} P \mathrm{PE}=1 \mathrm{SV}=1-$ [ACLY_MOUSE] & $0,000 E 0$ & $0,000 E 0$ & $0,000 E_{0}$ & $3,734 E 6$ \\
\hline Q9ET26 & E3 ubiquitin-protein ligase RNF114 OS=Mus musculus GN=Rnf144 PE=1 SV=2 - [RN114_MOUSE] & $0,000 E 0$ & $0,000 E 0$ & $0,000 E 0$ & $1,559 \mathrm{E} 6$ \\
\hline Q62312-2 & Isoform RII-1 of TGF-beta receptor type-2 OS=Mus musculus GN=Tgfbr2 - [TGFR2_MOUSE] & $0,000 E 0$ & $0,000 E 0$ & $0,000 \mathrm{E} 0$ & $3,425 E 6$ \\
\hline $008788-2$ & Isoform 2 of Dynactin subunit 1 OS=Mus musculus GN=Dctn1 - [DCTN1_MOUSE] & $0,000 \mathrm{E} 0$ & $0,000 E 0$ & $0,000 \mathrm{E} 0$ & $1,861 \mathrm{E} 6$ \\
\hline Q8C0C7 & Phenylalanine--tRNA ligase alpha subunit OS=Mus musculus GN=Farsa PE=1 SV=1 - [SYFA_MOUSE] & $0,000 E_{0}$ & $0,000 E 0$ & $0,0000_{0}$ & $2,208 E 6$ \\
\hline Q922D1 & Myotubularin-related protein $2 \mathrm{OS}=$ Mus musculus $\mathrm{GN}=\mathrm{Mtm} 2 \mathrm{PE}=1 \mathrm{SV}=3$ - [MTMR2_MOUSE] & $0,000 E 0$ & $0,000 E 0$ & $0,000 E 0$ & $8,915 E 5$ \\
\hline Q9CWX9 & Probable ATP-dependent RNA helicase DDX47 OS=Mus musculus GN=Ddx47 PE=2 SV=2 - [DDX47_MOUSE] & $0,000 E 0$ & $0,000 E_{0}$ & $0,000 E 0$ & 3,773E6 \\
\hline Q92289 & Ribosomal protein $\mathrm{S6}$ kinase alpha-4 OS=Mus musculus GN=Rps6ka4 PE=1 SV=2 - [KS6A4_MOUSE] & $0,000 E 0$ & $0,000 E 0$ & $0,000 E 0$ & $2,213 \mathrm{E} 6$ \\
\hline Q9CQU1 & Microfibrillar-associated protein 1 OS=Mus musculus GN=Mfap1 PE=1 SV=1 - [MFAP1_MOUSE] & $0,000 E 0$ & $0,000 E 0$ & $0,000 E 0$ & $6,425 E 5$ \\
\hline Q08943 & FACT complex subunit SSRP1 OS=Mus musculus GN=Ssrp1 PE=1 SV=2 - [SSRP1_MOUSE] & $0,000 E 0$ & $0,000 E 0$ & $0,000 E 0$ & 1,363E6 \\
\hline P31324 & cAMP-dependent protein kinase type II-beta regulatory subunit OS=Mus musculus GN=Prkar2b PE=1 $\mathrm{SV}=3$ - [KAP3_MOUSE] & $0,000 E_{0}$ & $0,000 E 0$ & $0,000 \mathrm{E} 0$ & $2,595 \mathrm{E} 6$ \\
\hline Q9DBE9 & pre-rRNA processing protein FTSJ3 OS=Mus musculus GN=Ftsj3 PE=1 SV=1- [SPB1_MOUSE] & $0,000 E_{0}$ & $0,000 E 0$ & $0,000 E_{0}$ & 1,958E6 \\
\hline P62996 & Transformer-2 protein homolog beta OS=Mus musculus GN=Tra2b PE=1 SV=1 - [TRA2B_MOUSE] & $0,000 E 0$ & $0,000 E 0$ & $0,000 E_{0}$ & $1,430 \mathrm{E} 7$ \\
\hline Q80UM7 & Mannosyl-oligosaccharide glucosidase OS=Mus musculus GN=Mogs PE=1 SV=1 - [MOGS_MOUSE] & $0,000 E 0$ & $0,000 E 0$ & $0,000 E 0$ & $2,944 \mathrm{E} 6$ \\
\hline P61021 & Ras-related protein Rab-5B OS=Mus musculus GN=Rab5b PE=1 SV=1 - [RAB5B_MOUSE] & $0,000 E 0$ & $0,000 E 0$ & 0,000E0 & 1,294E6 \\
\hline Q911433 & SH3 domain-containing protein 19 OS=Mus musculus GN=Sh3d19 PE=1 SV=2 - [SH319_MOUSE] & $0,000 E 0$ & $0,000 E 0$ & $0,000 E 0$ & $2,247 \mathrm{E}$ \\
\hline P28658 & Ataxin-10 OS=Mus musculus GN=Atxn10 PE=1 SV=2 - [ATX10_MOUSE] & $0,000 E_{0}$ & $0,000 E 0$ & $0,000 E_{0}$ & $3,189 \mathrm{E} 6$ \\
\hline Q922W5 & Pyyroline-5-carboxylate reductase 1, mitochondrial OS=Mus musculus GN=Pycr1 PE=1 SV=1 - [P5CR1_MOUSE] & $0,000 E 0$ & $0,000 E 0$ & $0,000 E 0$ & $3,839 \mathrm{E} 6$ \\
\hline Q9CWZ3-2 & Isoform 2 of RNA-binding protein 8A OS=Mus musculus GN=Rbm8a - [RBM8A_MOUSE] & $0,000 E 0$ & $0,000 E 0$ & $0,000 E 0$ & $1,669 \mathrm{EE}$ \\
\hline Q68FG3 & Protein SPT2 homolog OS=Mus musculus GN=Spty2d1 PE=2 SV=1 - [SPT2_MOUSE] & $0,000 E_{0}$ & $0,000 E 0$ & $0,000 \mathrm{E}_{0}$ & $2,451 E 6$ \\
\hline Q99kX6 & ADP-sugar pyrophosphatase OS=Mus musculus GN=Nudt5 PE=1 SV=1 - [NUDT5_MOUSE] & $0,000 E 0$ & $0,000 \mathrm{EO}$ & $0,000 E 0$ & 6,197E6 \\
\hline Q9D9Z1 & Small kinetochore-associated protein OS=Mus musculus GN=Knstrn PE=2 SV=2 - [SKAP_MOUSE] & $0,000 E 0$ & $0,0000_{0}$ & $0,000 \mathrm{E} 0$ & 3,112E6 \\
\hline B2RRE7 & OTU domain-containing protein $4 \mathrm{OS}=$ Mus musculus GN=Otud4 PE=1 $\mathrm{SV}=1$ - [OTUD4_MOUSE] & $0,000 E_{0}$ & $0,000 E 0$ & $0,000 E 0$ & 7,442E6 \\
\hline Q9D2Q3 & Uncharacterized protein C10orf88 homolog OS=Mus musculus PE=2 SV=1 - [C]088_MOUSE] & $0,000 E_{0}$ & $0,000 E 0$ & $0,000 E_{0}$ & $3,169 \mathrm{E} 6$ \\
\hline A2AQ25-8 & Isoform 8 of Sickle tail protein OS=Mus musculus GN=Skt - [SKT_MOUSE] & $0,000 E 0$ & $0,000 E 0$ & $0,000 E 0$ & $1,650 \mathrm{E} 6$ \\
\hline Q7TMF2 & 3'-5' exoribonuclease 1 OS=Mus musculus GN=Eri1 PE=1 SV=2 - [ERI1_MOUSE] & $0,000 E_{0}$ & $0,000 E 0$ & $0,000 \mathrm{E}^{-}$ & $1,411 \mathrm{E} 6$ \\
\hline Q8BG67 & Protein EFR3 homolog A OS=Mus musculus GN=Efr3a PE=1 SV=1 - [EFR3A_MOUSE] & $0,000 E_{0}$ & $0,000 \mathrm{EO}$ & $0,000 \mathrm{E}_{0}$ & $3,915 E 6$ \\
\hline$Q 62415$ & Apoptosis-stimulating of p53 protein 1 OS=Mus musculus GN=Ppp1r13b PE=1 SV=2 - [ASPP1_MOUSE] & $0,000 E 0$ & $0,000 E 0$ & $0,000 \mathrm{E} 0$ & 7,092E6 \\
\hline P35441 & Thrombospondin-1 OS=Mus musculus GN=Thbs1 PE=1 SV=1 - [TSP1_MOUSE] & $0,000 E 0$ & $0,000 E 0$ & $0,000 E_{0}$ & $8,753 E 6$ \\
\hline Q60749 & KH domain-containing, RNA-binding, signal transduction-associated protein 1 OS=Mus musculus GN=Khdrbs1 PE=1 SV=2 - [KHDR1_MOUSE] & $0,000 E 0$ & $0,000 E 0$ & $0,000 E_{0}$ & $1,327 \mathrm{E}$ \\
\hline Q91W50 & Cold shock domain-containing protein E1 OS=Mus musculus GN=CSde1 PE=1 SV=1 - [CSDE1_MOUSE] & $0,000 E_{0}$ & $0,000 E 0$ & $0,000 \mathrm{E}_{0}$ & $3,768 E 6$ \\
\hline Q9CR67 & Transmembrane protein 33 OS=Mus musculus GN=Tmem33 PE=1 SV=1 - [TMM33_MOUSE] & $0,000 E 0$ & $0,000 E 0$ & $0,000 \mathrm{E} 0$ & $1,557 \mathrm{E} 6$ \\
\hline Q62523 & Zyxin OS=Mus musculus GN=ZyX PE=1 SV=2 - [ZYX_MOUSE] & $0,000 E 0$ & $0,000 E_{0}$ & $0,000 E 0$ & $5,576 \mathrm{E} 6$ \\
\hline Q03958 & Prefoldin subunit 6 OS=Mus musculus GN=Pfdn6 PE=1 SV=1 - [PFD6_MOUSE] & $0,000 E 0$ & $0,000 E 0$ & $0,000 E 0$ & $1,502 E 6$ \\
\hline P54822 & Adenylosuccinate lyase OS=Mus musculus GN=AdsI PE=1 SV=2 - [PUR8_MOUSE] & $0,000 E_{0}$ & $0,000 E 0$ & $0,000 E_{0}$ & 1,150E6 \\
\hline Q64213-2 & Isoform CW17E of Splicing factor 1 OS=Mus musculus GN=Sf1 - [SF01_MOUSE] & $0,000 \mathrm{EO}$ & $0,000 E 0$ & $0,000 E 0$ & $4,830 \mathrm{E} 6$ \\
\hline
\end{tabular}




\begin{tabular}{|c|c|c|c|c|c|}
\hline Q80VD1 & Protein FAM98B OS=Mus musculus GN=Fam98b PE=1 SV=1 - [FA98B_MOUSE] & $0,000 \mathrm{E} 0$ & $0,000 \mathrm{E} 0$ & $0,000 E 0$ & $1,435 \mathrm{E} 6$ \\
\hline Q9WUA2 & Phenylalanine--tRNA ligase beta subunit OS=Mus musculus GN=Farsb PE=1 SV=2 - [SYFB_MOUSE] & $0,000 \mathrm{E} 0$ & $0,000 \mathrm{E} 0$ & $0,000 E 0$ & 3,032E6 \\
\hline Q3TZZ7 & Extended synaptotagmin-2 OS=Mus musculus GN=Esyt2 PE=1 SV=1 - [ESYT2_MOUSE] & $0,000 E 0$ & $0,000 E 0$ & $0,000 E 0$ & $4,744 E 6$ \\
\hline Q6NVF9 & Cleavage and polyadenylation specificity factor subunit 6 OS=Mus musculus $\mathrm{GN}=\mathrm{Cpsf6}$ PE=1 SV=1- [CPSF6_MOUSE] & $0,0000_{0}$ & $0,000 \mathrm{E} 0$ & $0,000 E 0$ & $5,040 \mathrm{E} 6$ \\
\hline P40336 & Vacuolar protein sorting-associated protein 26A OS=Mus musculus GN=Vps26a PE=1 SV=1 - [VP26A_MOUSE] & $0,000 E 0$ & $0,000 E 0$ & $0,000 E 0$ & 1,322E6 \\
\hline Q99M28-2 & Isoform 2 of RNA-binding protein with serine-rich domain 1 OS=Mus musculus GN=Rnps1 - [RNPS1_MOUSE] & $0,000 \mathrm{E} 0$ & $0,000 \mathrm{E} 0$ & $0,000 E 0$ & $2,831 E 6$ \\
\hline P27048 & Small nuclear ribonucleoprotein-associated protein B OS=Mus musculus GN=Snrpb PE=1 SV=1 - [RSMB_MOUSE] & $0,000 E_{0}$ & $0,000 E 0$ & $0,000 E 0$ & $2,612 \mathrm{E} 6$ \\
\hline Q80YR4-2 & Isoform 2 of Zinc finger protein 598 OS=Mus musculus GN=Znf598 - [ZN598_MOUSE] & $0,000 \mathrm{E} 0$ & $0,000 \mathrm{E} 0$ & $0,000 E 0$ & 7,897E5 \\
\hline Q03145 & Ephrin type-A receptor 2 OS=Mus musculus GN=Epha2 PE $=1 \mathrm{SV}=3-$ [EPHA2_MOUSE] & $0,000 \mathrm{E} 0$ & $0,000 E 0$ & $0,000 E 0$ & $9,608 \mathrm{E} 6$ \\
\hline E9Q3L2 & Phosphatidylinositol 4-kinase alpha OS=Mus musculus GN=Pi4ka PE=1 SV=2 - [PI4KA_MOUSE] & $0,000 \mathrm{E} 0$ & $0,000 \mathrm{E} 0$ & $0,000 E 0$ & $3,502 E 6$ \\
\hline Q80VI1 & E3 ubiquitin-protein ligase TRIM56 OS=Mus musculus GN=Trim56 PE=1 SV=1 - [TRI56_MOUSE] & $0,000 E 0$ & $0,000 E 0$ & $0,000 E 0$ & $4,133 \mathrm{E} 6$ \\
\hline Q5SUF2 & Luc7-like protein 3 OS=Mus musculus GN=LLC713 PE=1 SV=1 - [LC7L3_MOUSE] & $0,000 E_{0}$ & $0,000 \mathrm{E} 0$ & $0,000 E 0$ & $1,066 \mathrm{E} 7$ \\
\hline Q8BGU5-2 & Isoform 2 of Cyclin-Y OS=Mus musculus GN=CCny - [CCNY_MOUSE] & $0,000 \mathrm{E} 0$ & $0,000 E 0$ & $0,000 E 0$ & 1,658E6 \\
\hline Q6ZPF4 & Formin-like protein 3 OS=Mus musculus GN=FmnI3 PE=1 SV=2 - [FMNL__MOUSE] & $0,000 \mathrm{E}_{0}$ & $0,000 \mathrm{E} 0$ & $0,000 E 0$ & $5,625 \mathrm{E} 6$ \\
\hline Q8CH77 & Neuron navigator 1 OS=Mus musculus GN=Nav1 PE=1 SV=2 - [NAV1_MOUSE] & $0,000 \mathrm{E} 0$ & $0,000 \mathrm{E} 0$ & $0,000 E 0$ & $3,656 \mathrm{E}$ \\
\hline Q922R8 & Protein disulfide-isomerase A6 OS=Mus musculus GN=Pdia6 PE=1 SV=3 - [PDIA6_MOUSE] & $0,000 \mathrm{E} 0$ & $0,000 \mathrm{E} 0$ & $0,000 E 0$ & 4,343E6 \\
\hline P28828 & Receptor-type tyrosine-protein phosphatase mu OS=Mus musculus GN=Ptprm PE=1 SV=2 - [PTPRM_MOUSE] & $0,000 \mathrm{E} 0$ & $0,000 \mathrm{E} 0$ & $0,000 \mathrm{EO}$ & $1,893 \mathrm{E} 7$ \\
\hline Q8BTW9 & Serine/threonine-protein kinase PAK 4 OS=Mus musculus GN=Pak4 PE=1 SV=1 - [PAK4_MOUSE] & $0,000 E 0$ & $0,000 E 0$ & $0,000 E 0$ & $3,970 \mathrm{E} 6$ \\
\hline Q8BWT5 & Disco-interacting protein 2 homolog A OS=Mus musculus GN=Dip2a PE $=1 \mathrm{SV}=3$ - [DIP2A_MOUSE] & $0,000 E 0$ & $0,000 E 0$ & $0,000 E 0$ & $6,147 E 6$ \\
\hline E9Q6P5 & Tetratricopeptide repeat protein 7B OS=Mus musculus GN=Ttc7b PE=1 SV=1 - [TTC7B_MOUSE] & $0,000 \mathrm{E} 0$ & $0,000 \mathrm{E} 0$ & $0,000 E 0$ & $1,440 \mathrm{E} 6$ \\
\hline Q60973 & Histone-binding protein RBBP7 OS=Mus musculus GN=Rbbp7 PE=1 SV=1 - [RBBP7_MOUSE] & $0,000 \mathrm{E} 0$ & $0,000 \mathrm{E} 0$ & $0,000 E 0$ & 5,163E6 \\
\hline Q8BHL3 & TBC1 domain family member 10B OS=Mus musculus GN=Tbc1d10b PE=1 SV=2 - [TB10B_MOUSE] & $0,000 \mathrm{E} 0$ & $0,000 \mathrm{E} 0$ & $0,000 E 0$ & 3,973E6 \\
\hline Q8VDM6-2 & Isoform 2 of Heterogeneous nuclear ribonucleoprotein U-like protein 1 OS=Mus musculus GN=Hnrnpul1 - [HNRL1_MOUSE] & $0,000 E 0$ & $0,000 E 0$ & $0,000 E 0$ & 3,663E6 \\
\hline Q8C854-2 & Isoform 4 of Myelin expression factor 2 OS=Mus musculus GN=Myef2 - [MYEF2_MOUSE] & $0,000 E_{0}$ & $0,000 \mathrm{E} 0$ & $0,000 E 0$ & $2,791 \mathrm{E} 6$ \\
\hline Q3UFK8 & FERM domain-containing protein 8 OS=Mus musculus GN=Frmd8 PE=1 SV=2 - [FRMD8_MOUSE] & $0,000 E 0$ & $0,000 E 0$ & $0,000 E 0$ & 1,696E6 \\
\hline Q5EG47 & 5'-AMP-activated protein kinase catalytic subunit alpha-1 OS=Mus musculus GN=Prkaa1 PE=1 SV=2 - [AAPK1_MOUSE] & $0,000 \mathrm{E} 0$ & $0,000 \mathrm{E} 0$ & $0,000 E 0$ & $3,574 E 6$ \\
\hline Q78ZA7 & Nucleosome assembly protein 1-like 4 OS=Mus musculus GN=Nap114 PE=1 SV=1 - [NP1L4_MOUSE] & $0,000 \mathrm{E} 0$ & $0,000 \mathrm{E} 0$ & $0,000 E 0$ & $2,814 E 6$ \\
\hline Q8CJ53-4 & Isoform 4 of Cdc42-interacting protein 4 OS=Mus musculus GN=Trip10 - [CIP4_MOUSE] & $0,000 \mathrm{E} 0$ & $0,000 \mathrm{E} 0$ & $0,000 E 0$ & $2,371 \mathrm{E}$ \\
\hline Q9D1E6 & Tubulin-folding cofactor B OS=Mus musculus GN=Tbcb PE=1 SV=2 - [TBCB_MOUSE] & $0,000 \mathrm{E} 0$ & $0,000 \mathrm{E} 0$ & $0,000 E 0$ & $1,996 \mathrm{E} 6$ \\
\hline Q91064 & Isochorismatase domain-containing protein 1 OS=Mus musculus GN=Isoc1 PE=1 SV=1 - [ISOC1_MOUSE] & $0,000 E 0$ & $0,000 E 0$ & $0,000 E 0$ & $6,309 \mathrm{E} 5$ \\
\hline Q9C230 & Obg-like ATPase 1 OS=Mus musculus GN=Ola1 PE=1 SV=1 - [OLA1_MOUSE] & $0,000 E 0$ & $0,000 E 0$ & $0,000 E 0$ & $5,487 \mathrm{E} 6$ \\
\hline 008810 & $116 \mathrm{kDa}$ U5 small nuclear ribonucleoprotein component OS=Mus musculus GN=Eftud2 PE=1 SV=1 - [U5S1_MOUSE] & $0,000 \mathrm{E} 0$ & $0,000 \mathrm{E} 0$ & $0,000 E 0$ & 1,263E6 \\
\hline P70261 & Paladin OS=Mus musculus GN=Pald1 PE=1 SV=1 - [PALD_MOUSE] & $0,000 \mathrm{E} 0$ & $0,000 \mathrm{E} 0$ & $0,000 E 0$ & $3,772 \mathrm{E} 6$ \\
\hline P61620 & Protein transport protein Sec61 subunit alpha isoform 1 OS=Mus musculus GN=Sec61a1 PE=1 SV=2 - [S61A1_MOUSE] & $0,000 E_{0}$ & $0,000 \mathrm{E} 0$ & $0,000 E 0$ & $8,238 E 6$ \\
\hline Q3UMU9-4 & Isoform 4 of Hepatoma-derived growth factor-related protein 2 OS=Mus musculus GN=Hdgfrp2 - [HDGR2_MOUSE] & $0,000 E 0$ & $0,000 E 0$ & $0,000 E 0$ & 7,439E6 \\
\hline Q8C166 & Copine-1 OS=Mus musculus GN=CPne1 PE=1 SV=1 - [CPNE1_MOUSE] & $0,000 \mathrm{E} 0$ & $0,000 E 0$ & $0,000 E 0$ & $3,143 E 6$ \\
\hline P05532-2 & Isoform 2 of Mast//stem cell growth factor receptor Kit OS=Mus musculus GN=Kit - [KIT_MOUSE] & $0,000 \mathrm{E} 0$ & $0,000 \mathrm{E} 0$ & $0,000 E 0$ & 6,934E6 \\
\hline P24788-2 & Isoform 2 of Cyclin-dependent kinase 11B OS=Mus musculus GN=Cdk11b - [CD11B_MOUSE] & $0,000 \mathrm{E} 0$ & $0,000 \mathrm{E} 0$ & $0,000 E 0$ & $2,265 E 6$ \\
\hline Q8BTH8 & Casein kinase I isoform gamma-1 OS=Mus musculus GN=CSnk1g1 PE=1 SV=2 - [KC1G1_MOUSE] & $0,000 \mathrm{E} 0$ & $0,000 \mathrm{E} 0$ & $0,000 E 0$ & 1,557E6 \\
\hline Q8BSF4 & Phosphatidylserine decarboxylase proenzyme, mitochondrial OS=Mus musculus GN=Pisd PE=2 SV=1 - [PISD_MOUSE] & $0,000 \mathrm{E} 0$ & $0,000 \mathrm{E} 0$ & $0,000 E 0$ & $1,467 \mathrm{E} 6$ \\
\hline Q80rwo & Cytohesin-4 OS=Mus musculus GN=Cyth4 PE=1 SV=1 - [CYH4_MOUSE] & $0,000 \mathrm{E} 0$ & $0,000 \mathrm{E} 0$ & $0,000 E 0$ & $6,843 \mathrm{E} 6$ \\
\hline Q8BGQ7 & Alanine--tRNA ligase, cytoplasmic OS=Mus musculus GN=Aars PE=1 SV=1 - [SYAC_MOUSE] & $0,000 E 0$ & $0,000 E 0$ & $2,770 \mathrm{E} 6$ & $1,262 E 6$ \\
\hline P54103 & Dna] homolog subfamily C member 2 OS=Mus musculus GN=Dnajc2 PE=1 SV=2 - [DNJC2_MOUSE] & $0,000 \mathrm{E} 0$ & $0,000 \mathrm{E} 0$ & $0,000 E 0$ & $2,778 E 6$ \\
\hline Q9Z130 & Heterogeneous nuclear ribonucleoprotein D-like OS=Mus musculus GN=Hnrnpdl PE=1 SV=1 - [HNRDL_MOUSE] & $0,000 \mathrm{E} 0$ & $0,000 \mathrm{E} 0$ & $0,000 E 0$ & $2,962 E 6$ \\
\hline 070591 & Prefoldin subunit $20 S=$ Mus musculus $\mathrm{GN}=\mathrm{Pfdn} 2 \mathrm{PE}=1 \mathrm{SV}=2$ - [PFD2_MOUSE] & $0,000 E_{0}$ & $0,000 \mathrm{E} 0$ & $0,000 E 0$ & $2,267 \mathrm{E}$ \\
\hline Q99MU3-2 & Isoform 2 of Double-stranded RNA-specific adenosine deaminase OS=Mus musculus GN=Adar - [DSRAD_MOUSE] & $0,000 E 0$ & $0,000 E 0$ & $0,0000_{0}$ & $1,586 \mathrm{E} 6$ \\
\hline Q922M3 & BTB/POZ domain-containing adapter for CUL3-mediated RhoA degradation protein 3 OS=Mus musculus GN=Kctd10 PE=1SV=1 - [BACD3_MOUSE] & $0,000 E 0$ & $0,000 E 0$ & $0,000 E 0$ & $1,638 \mathrm{E} 6$ \\
\hline Q9Z2N8 & Actin-like protein $6 \mathrm{~A}$ OS=Mus musculus GN=Actl6a PE=1 SV=2 - [ACL6A_MOUSE] & $0,000 E 0$ & $0,000 E 0$ & $0,000 E 0$ & $1,781 \mathrm{E} 6$ \\
\hline Q8R0S2 & IQ motif and SEC7 domain-containing protein $1 \mathrm{OS}=$ Mus musculus $\mathrm{GN}=\mathrm{I}$ qsec1 PE=1 SV=2 - [IQEC1_MOUSE] & $0,000 \mathrm{E} 0$ & $0,000 E 0$ & $0,000 E 0$ & 9,196E6 \\
\hline P63158 & High mobility group protein B1 OS=Mus musculus GN=Hmgb1 PE=1 SV=2 - [HMGB1_MOUSE] & $0,000 E 0$ & $0,000 E 0$ & $1,350 \mathrm{E} 6$ & $5,490 \mathrm{E} 6$ \\
\hline Q8C4X2 & Casein kinase I isoform gamma-3 OS=Mus musculus $\mathrm{GN}=\mathrm{CSnk} 193 \mathrm{PE}=1 \mathrm{SV}=2$ - [KC1G__MOUSE] & $0,000 \mathrm{E} 0$ & $0,000 \mathrm{E} 0$ & $0,000 E 0$ & $8,887 \mathrm{E}$ \\
\hline Q8COT5-2 & Isoform 2 of Signal-induced proliferation-associated 1-like protein 1 OS=Mus musculus GN=Sipa111 - [SIL1__MOUSE] & $0,000 \mathrm{E} 0$ & $0,000 \mathrm{E} 0$ & $0,000 E 0$ & $2,472 E 6$ \\
\hline 054941 & SWI/SNF-related matrix-associated actin-dependent regulator of chromatin subfamily E member $10 \mathrm{OS}=$ Mus musculus GN=Smarce1 PE=1SV=1 - [SMCE1_MOUSE] & 0,000E0 & 0,000E0 & $0,000 E 0$ & $1,832 \mathrm{E} 6$ \\
\hline Q56925 & Probable ATP-dependent RNA helicase DDX46 OS=Mus musculus GN=Ddx46 PE=1 SV=2 - [DDX46_MOUSE] & $0,000 E 0$ & $0,000 E 0$ & $0,000 E 0$ & $1,133 \mathrm{E} 6$ \\
\hline Q99447 & Hsc70-interacting protein OS=Mus musculus GN=St13 PE=1 SV=1 - [F10A1_MOUSE] & $0,000 \mathrm{E} 0$ & $0,000 \mathrm{E} 0$ & $0,000 E 0$ & $3,360 \mathrm{E} 6$ \\
\hline Q68FF6 & ARF GTPase-activating protein GIT1 OS=Mus musculus GN=Git1 PE=1 SV=1 - [GIT1_MOUSE] & $0,000 \mathrm{E} 0$ & $0,000 E 0$ & $0,000 E 0$ & $2,866 \mathrm{E} 6$ \\
\hline Q8C108 & SLAIN motif-containing protein 2 OS=Mus musculus GN=Slain2 PE=1 SV=2 - [SLAI2_MOUSE] & $0,0000_{0}$ & 0,000E0 & $0,000 E 0$ & $1,662 \mathrm{E} 6$ \\
\hline Q9EQU5-2 & Isoform 2 of Protein SET OS=Mus musculus GN=Set - [SET_MOUSE] & $0,000 E 0$ & $0,000 E 0$ & $0,000 E 0$ & $1,135 E 6$ \\
\hline P40142 & Transketolase OS=Mus musculus GN=TKt PE=1 SV=1 - [TKT_MOUSE] & $0,000 \mathrm{E}_{0}$ & $0,000 \mathrm{E} 0$ & $0,000 E 0$ & $1,552 \mathrm{E} 6$ \\
\hline P28740 & Kinesin-like protein KIF2A OS=Mus musculus GN=Kifza PE $=1 \mathrm{SV}=2-$ [KIF2A_MOUSE] & $0,000 \mathrm{E} 0$ & $0,000 \mathrm{E} 0$ & $0,000 E 0$ & $3,446 E 6$ \\
\hline P63073 & Eukaryotic translation initiation factor $4 \mathrm{E}$ OS=Mus musculus GN=Eif4e PE=1 SV=1 - [IF4E_MOUSE] & $0,000 E 0$ & $0,000 E 0$ & $0,000 E 0$ & $1,825 \mathrm{E} 6$ \\
\hline Q69ZR2 & E3 ubiquitin-protein ligase HECTD1 OS=Mus musculus GN=Hectd1 PE $=1 \mathrm{SV}=2$ - [HECD1_MOUSE] & $0,000 \mathrm{E} 0$ & $0,000 \mathrm{E} 0$ & $0,000 E 0$ & $4,761 \mathrm{E}$ \\
\hline Q62376 & U1 small nuclear ribonucleoprotein $70 \mathrm{kDa}$ OS=Mus musculus GN=Snrnp70 PE=1 SV=2 - [RU17_MOUSE] & $0,000 E 0$ & $0,000 E 0$ & $0,000 E 0$ & $3,976 \mathrm{E} 6$ \\
\hline Q9DC70 & NADH dehydrogenase [ubiquinone] iron-sulfur protein 7, mitochondrial OS=Mus musculus GN=Ndufs7 PE=1 SV=1 - [NDUS7_MOUSE] & $0,000 E 0$ & $0,000 E 0$ & $0,000 E 0$ & $1,121 \mathrm{E} 6$ \\
\hline 009110 & Dual specificity mitogen-activated protein kinase kinase 3 OS=Mus musculus GN=Map2k3 PE=1 SV=2 - [MP2K3_MOUSE] & $0,000 E 0$ & $0,000 E 0$ & $0,000 E 0$ & 3,822E6 \\
\hline Q309G9 & Lamin-B receptor OS=Mus musculus GN=Lbr PE=1 SV=2 - [LBR_MOUSE] & $0,000 \mathrm{E} 0$ & $0,000 \mathrm{E} 0$ & $0,000 E 0$ & $9,738 E 6$ \\
\hline Q8CH72 & E3 ubiquitin-protein ligase TRIM32 OS=Mus musculus GN=Trim32 PE=1 SV=2 - [TRI32_MOUSE] & $0,000 E_{0}$ & $0,000 E_{0}$ & $0,000 E 0$ & 6,924E6 \\
\hline Q8BN32 & A disintegrin and metalloproteinase with thrombospondin motiff $4 \mathrm{OS}=$ Mus musculus $\mathrm{GN}=\mathrm{Adamt5} 4 \mathrm{PE}=2 \mathrm{SV}=2-$ [ATS4_MOUSE] & $0,000 \mathrm{E} 0$ & $0,000 \mathrm{E} 0$ & $0,000 E 0$ & 1,303E7 \\
\hline E9Q555 & E3 ubiquitin-protein ligase RNF213 OS=Mus musculus GN=Rnf213 PE=1 SV=2 - [RN213_MOUSE] & $0,000 \mathrm{E} 0$ & $0,000 \mathrm{E} 0$ & $8,642 E 5$ & $1,198 \mathrm{E} 7$ \\
\hline Q60790 & Ras GTPase-activating protein 3 OS=Mus musculus GN=Rasa3 PE=1 SV=2 - [RASA3_MOUSE] & $0,000 \mathrm{E} 0$ & $0,000 \mathrm{E} 0$ & $0,000 E 0$ & $3,180 \mathrm{E} 6$ \\
\hline Q8BHD7-2 & Isoform 2 of Polypyrimidine tract-binding protein 3 OS=Mus musculus GN=Ptbp3 - [PTBP3_MOUSE] & $0,000 \mathrm{E} 0$ & $0,000 \mathrm{E} 0$ & $0,000 E 0$ & $3,256 \mathrm{E} 7$ \\
\hline P17095-1 & Isoform HMG-Y of High mobility group protein HMG-I/HMG-Y OS=Mus musculus GN=Hmga1 - [HMGA1_MOUSE] & $0,000 \mathrm{E} 0$ & $0,000 \mathrm{E} 0$ & $0,000 E 0$ & $8,704 E 7$ \\
\hline Q8BH88 & DEP domain-containing protein 1B OS=Mus musculus GN=Depdc1b PE=2 SV=1 - [DEP1B_MOUSE] & $0,000 \mathrm{E} 0$ & $0,000 \mathrm{E} 0$ & $0,000 E 0$ & $3,815 E 5$ \\
\hline Q3TTV5 & Zinc finger CCCH domain-containing protein 15 OS=Mus musculus GN=ZC3h15 PE=1 SV=2 - [ZC3HF_MOUSE] & $0,000 \mathrm{E} 0$ & $0,000 \mathrm{E} 0$ & $0,000 E 0$ & $5,932 E 6$ \\
\hline Q8CGC7 & Bifunctional glutamate/proline--tRNA ligase OS=MUs musculus GN=Eprs PE=1 SV=4 - [SYEP_MOUSE] & $0,000 E 0$ & $0,000 E 0$ & $0,000 E 0$ & $5,461 E 6$ \\
\hline Q64737 & Trifunctional purine biosynthetic protein adenosine-3 $3 \mathrm{OS}=$ Mus musculus $\mathrm{GN}=\mathrm{Gart} \mathrm{PE}=1 \mathrm{SV}=3$ - [PUR2_MOUSE] & $0,000 \mathrm{E} 0$ & $0,000 \mathrm{E} 0$ & $0,000 E 0$ & $5,563 \mathrm{E} 6$ \\
\hline Q92208 & C-1-tetrahydrofolate synthase, cytoplasmic OS=Mus musculus $\mathrm{GN}=$ Mthfd1 $\mathrm{PE}=1 \mathrm{SV}=4$ - [C1TC_MOUSE] & $0,000 \mathrm{E} 0$ & $0,000 \mathrm{E} 0$ & $0,000 E 0$ & $2,757 \mathrm{E}$ \\
\hline Q9ESL4-2 & Isoform 2 of Mitogen-activated protein kinase kinase kinase MLT OS=Mus musculus GN=Zak - [MLTK_MOUSE] & $0,000 E 0$ & $0,000 \mathrm{E} 0$ & $0,000 E 0$ & $2,924 E 6$ \\
\hline Q9R0E1 & Procollagen-lysine, 2 -oxoglutarate 5-dioxygenase $3 \mathrm{OS}=$ Mus musculus $\mathrm{GN}=P$ Plod3 PE=1 SV=1 - [PLOD3_MOUSE] & $0,000 E_{0}$ & $0,000 E 0$ & $0,000 E 0$ & $1,599 \mathrm{E} 6$ \\
\hline Q60953-2 & Isoform 2 of Protein PML OS=Mus musculus GN=PmI - [PML_MOUSE] & $0,000 \mathrm{E} 0$ & $0,000 \mathrm{E} 0$ & $0,000 E 0$ & $5,147 E 6$ \\
\hline Q8BWY3 & Eukaryotic peptide chain release factor subunit 1 OS=Mus musculus GN=Etf1 PE=1 SV=4 - [ERF1_MOUSE] & $0,000 \mathrm{E} 0$ & $0,000 \mathrm{E} 0$ & $0,000 E 0$ & $2,605 E 6$ \\
\hline Q9CZ52 & Anthrax toxin receptor 1 OS=Mus musculus GN=Antxr1 PE=1 SV=2 - [ANTR1_MOUSE] & $0,000 \mathrm{E} 0$ & $0,000 E 0$ & $0,000 E 0$ & $5,248 E 6$ \\
\hline P62046 & Leucine-rich repeat and calponin homology domain-containing protein 1 OS=Mus musculus GN=Lrch1 PE=1 SV=2 - [LRCH1_MOUSE] & $0,000 \mathrm{E} 0$ & $0,000 \mathrm{E} 0$ & $0,000 E 0$ & $4,710 \mathrm{E} 7$ \\
\hline Q8K363 & ATP-dependent RNA helicase DDX18 OS=Mus musculus GN=Ddx18 PE=1 SV=1 - [DDX18_MOUSE] & $0,000 \mathrm{E} 0$ & $0,000 \mathrm{E} 0$ & $0,000 E 0$ & $2,499 \mathrm{E} 6$ \\
\hline Q9ERU9 & E3 SUMO-protein ligase RanBP2 OS=Mus musculus GN=Ranbp2 PE=1 SV=2 - [RBP2_MOUSE] & $0,000 \mathrm{E} 0$ & $0,000 \mathrm{E} 0$ & $0,000 E 0$ & $3,740 \mathrm{E} 6$ \\
\hline 035691 & Pinin OS=Mus musculus GN=Pnn PE=1 SV=4 - [PININ_MOUSE] & $0,000 \mathrm{E} 0$ & $0,000 \mathrm{E} 0$ & $0,000 E 0$ & $3,341 E 6$ \\
\hline P70255-5 & Isoform 5 of Nuclear factor 1 C-type OS=Mus musculus GN=Nfic - [NFIC_MOUSE] & $0,000 \mathrm{E} 0$ & $0,000 \mathrm{E} 0$ & $0,000 E 0$ & $8,960 \mathrm{E} 6$ \\
\hline Q7TN60 & Transmembrane channel-like protein 6 OS=Mus musculus GN=Tmc6 PE=1 SV=2 - [TMC6_MOUSE] & $0,000 \mathrm{E} 0$ & $0,000 \mathrm{E} 0$ & $0,000 E 0$ & $4,582 E 6$ \\
\hline Q8CESO & N-alpha-acetyltransferase $30 \mathrm{OS}=$ Mus musculus $\mathrm{GN}=\mathrm{Naa} 30 \mathrm{PE}=1 \mathrm{SV}=2$ - [NAA30_MOUSE] & $0,000 \mathrm{E} 0$ & $0,000 \mathrm{E} 0$ & $0,000 E 0$ & $2,084 E 6$ \\
\hline P05132-2 & Isoform 2 of cAMP-dependent protein kinase catalytic subunit alpha OS=Mus musculus GN=Prkaca - [KAPCA_MOUSE] & $0,000 E_{0}$ & $0,000 \mathrm{E} 0$ & $0,000 E 0$ & $7,310 \mathrm{E} 6$ \\
\hline P70429-2 & Isoform 1 of Ena/VASP-like protein OS=Mus musculus GN=Evl - [EVL_MOUSE] & $0,000 \mathrm{E} 0$ & $0,000 \mathrm{E} 0$ & $0,000 E 0$ & $1,947 \mathrm{E} 6$ \\
\hline Q3UMG5 & Leucine-rich repeat and calponin homology domain-containing protein 2 OS=Mus musculus $\mathrm{GN}=$ Lrch2 PE $=2 \mathrm{SV}=2$ - [LRCH2_MOUSE] & $0,000 \mathrm{E} 0$ & $0,000 \mathrm{E} 0$ & $0,000 E 0$ & $2,261 \mathrm{E} 6$ \\
\hline Q62432-2 & Isoform Short of Mothers against decapentaplegic & $0,000 E 0$ & $0,000 E 0$ & $0,000 E 0$ & $1,545 \mathrm{E} 6$ \\
\hline
\end{tabular}




\begin{tabular}{|c|c|c|c|c|c|}
\hline Q791155 & Mitochondrial carrier homolog $2 \mathrm{OS}=$ Mus musculus $\mathrm{GN}=$ Mtch2 PE=1 $\mathrm{SV}=1$ - [MTCH2_MOUSE] & $0,000 E_{0}$ & $0,000 E_{0}$ & $0,000 \mathrm{E}_{0}$ & $2,528 E 6$ \\
\hline P28740-2 & Isoform 2 of Kinesin-like protein KIF2A OS=Mus musculus GN=Kifza - [KIF2A_MOUSE] & $0,000 E_{0}$ & $0,000 E_{0}$ & $0,000 \mathrm{E}_{0}$ & $3,446 \mathrm{E} 6$ \\
\hline Q6PGHO & Ubiquitin domain-containing protein 2 OS=Mus musculus GN=Ubtd2 PE=2 SV=1 - [UBTD2_MOUSE] & $0,000 E_{0}$ & $0,000 E 0$ & $0,000 E_{0}$ & $1,088 E 6$ \\
\hline Q91VZ6 & Stromal membrane-associated protein 1 OS=Mus musculus GN=Smap1 PE=1 SV=1 - [SMAP1_MOUSE] & $0,000 E 0$ & $0,000 E 0$ & $0,000 E 0$ & 7,338E6 \\
\hline Q8K310 & Matrin-3 OS=Mus musculus GN=Matr3 PE=1 SV=1 - [MATR3_MOUSE] & $0,000 E_{0}$ & $0,000 \mathrm{E} 0$ & $0,000 E 0$ & $3,095 \mathrm{E} 6$ \\
\hline Q9R1C7 & Pre-mRNA-processing factor 40 homolog A OS=Mus musculus GN=Prpf40a PE=1 SV=1 - [PR40A_MOUSE] & $0,000 E_{0}$ & $0,000 E_{0}$ & $0,000 \mathrm{E}_{0}$ & 3,490E6 \\
\hline Q8BP48 & Methionine aminopeptidase $1 \mathrm{OS}=$ Mus musculus GN=Metap1 PE=1 SV=1 - [MAP11_MOUSE] & $0,000 E 0$ & $0,000 E 0$ & $0,000 E 0$ & $5,609 \mathrm{E} 6$ \\
\hline Q922H2 & [Pyruvate dehydrogenase (acetyl-transferring)] kinase isozyme 3, mitochondrial OS=Mus musculus GN=Pdk3 PE=1 SV=1 - [PDK3_MOUSE] & $0,000 E 0$ & $0,000 E_{0}$ & $0,000 E 0$ & $1,334 E 6$ \\
\hline $089032-3$ & Isoform 3 of SH3 and PX domain-containing protein 2A OS=Mus musculus GN=Sh3pxd2a - [SPD2A_MOUSE] & $0,000 E 0$ & $0,000 E 0$ & $0,000 E 0$ & $1,672 E 6$ \\
\hline QTTNVO & Protein DEK OS=Mus musculus GN=Dek PE=1 SV=1 - [DEK_MOUSE] & $0,000 E_{0}$ & $0,000 E_{0}$ & $0,000 E_{0}$ & 9,174E6 \\
\hline P58742 & Aladin $\mathrm{OS}=$ Mus musculus GN=Aaas PE=1 SV=1 - [AAAS_MOUSE] & $0,000 E 0$ & $0,000 E 0$ & $0,000 E 0$ & $2,053 \mathrm{E} 6$ \\
\hline Q922B9 & Sperm-specific antigen 2 homolog OS=Mus musculus GN=Ssfa2 PE=1 SV=3 - [SSFA2_MOUSE] & $0,000 E 0$ & $0,000 E_{0}$ & $0,000 \mathrm{E}_{0}$ & $1,922 \mathrm{E} 6$ \\
\hline Q9CX56 & 26S proteasome non-ATPase regulatory subunit 8 OS=Mus musculus GN=Psmd8 PE=1 SV=2 - [PSMD8_MOUSE] & $0,000 E_{0}$ & $0,000 E_{0}$ & $0,000 E 0$ & $1,638 E 6$ \\
\hline Q9DOR8 & Protein LSM12 homolog OS=Mus musculus GN=LSm12 PE=1 SV=1 - [LSM12_MOUSE] & $0,000 E 0$ & $0,000 E_{0}$ & $0,000 E 0$ & 6,599E6 \\
\hline P52479 & Ubiquitin carboxyl-terminal hydrolase 10 OS=Mus musculus GN=Usp10 PE=1 SV=3 - [UBP10_MOUSE] & $0,000 E 0$ & $0,000 E 0$ & $0,000 E 0$ & $5,551 \mathrm{E} 6$ \\
\hline E9QAM5 & Helicase with zinc finger domain 2 OS=Mus musculus GN=Helz2 PE=1 SV=1 - [HELZ2_MOUSE] & $0,000 \mathrm{E} 0$ & $0,000 \mathrm{E} 0$ & $0,000 E 0$ & 4,552E6 \\
\hline 99857 & A disintegrin and metalloproteinase with thrombospondin motifs $1 \mathrm{OS}=$ Mus musculus GN=Adamts1 PE=1 SV=4 - [ATS1_MOUSE] & $0,000 E 0$ & $0,000 E 0$ & $0,000 E 0$ & $1,311 \mathrm{EE}$ \\
\hline Q91WF3 & Adenylate cyclase type 4 OS=Mus musculus GN=Adcy4 $\mathrm{PE}=1 \mathrm{SV}=1$ - [ADCY4_MOUSE] & $0,000 E_{0}$ & $0,000 E_{0}$ & $0,000 E 0$ & $1,978 E 7$ \\
\hline Q9QY06 & Unconventional myosin-IXb OS=Mus musculus GN=Myogb PE=1 SV=2 - [MYO9B_MOUSE] & $0,000 E 0$ & $0,000 E 0$ & $0,000 E 0$ & $1,760 \mathrm{E} 7$ \\
\hline Q60930 & Voltage-dependent anion-selective channel protein 2 OS=Mus musculus GN=Vdac2 PE=1 SV=2 - [VDAC2_MOUSE] & $0,000 \mathrm{E} 0$ & $0,000 E 0$ & $0,000 \mathrm{E} 0$ & $2,949 \mathrm{E} 6$ \\
\hline Q61211 & Eukaryotic translation initiation factor $2 \mathrm{D}$ OS=Mus musculus GN=Eif2d PE=1 SV=3 - [EIF2D_MOUSE] & $0,000 E_{0}$ & $0,000 E_{0}$ & $0,000 \mathrm{E} 0$ & $1,481 E 6$ \\
\hline P52624 & Uridine phosphorylase 1 OS=Mus musculus GN=Upp1 PE=1 SV=2 - [UPP1_MOUSE] & $0,000 E 0$ & $0,000 \mathrm{E} 0$ & $0,000 E 0$ & $1,649 \mathrm{E}$ \\
\hline P31938 & Dual specificity mitogen-activated protein kinase kinase $1 \mathrm{OS}=$ Mus musculus GN=Map2k1 PE=1 SV=2 - [MP2K1_MOUSE] & $0,000 E 0$ & $0,000 E 0$ & $0,000 E 0$ & $2,900 \mathrm{E} 6$ \\
\hline Q9QUJ7-2 & Isoform Short of Long-chain-fatty-acid--CoA ligase 4 OS=Mus musculus GN=Acs14 - [ACSL4_MOUSE] & $0,000 E 0$ & $0,000 E 0$ & $0,000 E 0$ & $1,513 \mathrm{EE}$ \\
\hline Q8K2Ү3 & Protein eva-1 homolog B OS=Mus musculus GN=Eva1b PE=1 SV=1 - [EVA1B_MOUSE] & $0,000 E_{0}$ & $0,000 E_{0}$ & $0,000 E_{0}$ & 7,679E6 \\
\hline P61290 & Proteasome activator complex subunit 3 OS=Mus musculus GN=Psme3 $\mathrm{PE}=1 \mathrm{SV}=1$ - [PSME3_MOUSE] & $0,000 E_{0}$ & $0,000 E_{0}$ & $0,000 E 0$ & $9,215 E 5$ \\
\hline Q64282 & Interferon-induced protein with tetratricopeptide repeats 1 OS=Mus musculus GN=Ifit1 PE=1 $1 \mathrm{VV}=2$ - [IFIT1_MOUSE] & $0,000 E_{0}$ & $0,000 E_{0}$ & $0,000 E 0$ & $2,261 E 6$ \\
\hline Q6ZQ08-2 & Isoform 2 of CCR4-NOT transcription complex subunit 1 OS=Mus musculus GN=Cnot1 - [CNOT1_MOUSE] & $0,000 E_{0}$ & $0,000 E_{0}$ & $0,000 \mathrm{E} 0$ & $3,243 E 6$ \\
\hline B2RXS4 & Plexin-B2 OS=Mus musculus GN=Plxnb2 PE=1 SV=1 - [PLXB2_MOUSE] & $0,000 E_{0}$ & $0,000 E_{0}$ & $0,000 E 0$ & $8,971 E 6$ \\
\hline Q8BYZ1 & ABI gene family member $3 \mathrm{OS}=$ Mus musculus $\mathrm{GN}=\mathrm{Abi3} \mathrm{PE}=1 \mathrm{SV}=3$ - [ABI3_MOUSE] & $0,000 E_{0}$ & $0,000 E 0$ & $0,000 E 0$ & $1,460 \mathrm{E} 6$ \\
\hline Q9DBG6 & Dolichyl-diphosphooligosaccharide--protein glycosyltransferase subunit $2 \mathrm{OS}=$ Mus musculus GN=Rpn2 PE=1 SV=1- [RPN2_MOUSE] & $0,000 E 0$ & $0,000 E 0$ & $0,000 E 0$ & $4,305 E 6$ \\
\hline P70290 & $55 \mathrm{kDa}$ erythrocyte membrane protein OS=Mus musculus GN=Mpp1 PE=1 SV=1 - [EM55_MOUSE] & $0,000 E_{0}$ & $0,000 E_{0}$ & $0,000 \mathrm{E}_{0}$ & $1,230 \mathrm{E} 6$ \\
\hline Q6AOA2-2 & Isoform 2 of La-related protein 4B OS=Mus musculus GN=Larp4b - [LAR4B_MOUSE] & $0,000 E 0$ & $0,000 \mathrm{E} 0$ & $0,000 E 0$ & $2,613 \mathrm{EE}$ \\
\hline Q8BGH2 & Sorting and assembly machinery component 50 homolog OS=Mus musculus GN=Samm50 PE=1 SV=1 - [SAM50_MOUSE] & $0,000 E_{0}$ & $0,000 E_{0}$ & $0,000 E 0$ & $1,496 \mathrm{E} 6$ \\
\hline $\mathrm{P} 46662-2$ & Isoform 2 of Merlin OS=Mus musculus GN=N22 - [MERL_MOUSE] & $0,000 E 0$ & $0,000 E 0$ & $0,000 E 0$ & $3,500 \mathrm{E} 6$ \\
\hline Q91XI1 & tRNA-dihydrouridine(47) synthase [NAD(P)(+)]-like OS=Mus musculus GN=Dus31 PE=1 SV=1 - [DUS3L_MOUSE] & $0,000 E 0$ & $0,000 E 0$ & $0,000 E 0$ & $2,557 \mathrm{E} 6$ \\
\hline Q99641 & EMILIN-1 OS=Mus musculus GN=Emilin1 PE=1 SV=1 - [EMIL1_MOUSE] & $0,000 E 0$ & $0,000 E 0$ & $0,000 E 0$ & 3,541E6 \\
\hline P23298 & Protein kinase C eta type OS=Mus musculus GN=Prkch PE=1 SV=2 - [KPCL_MOUSE] & $0,000 E 0$ & $0,000 E 0$ & $0,000 E 0$ & $1,263 E 6$ \\
\hline Q8K297 & Procollagen galactosyltransferase $10 \mathrm{OS}=$ Mus musculus GN=Colgalt1 PE=1 SV=2 - [GT251_MOUSE] & $0,000 E 0$ & $0,000 E 0$ & $0,000 E 0$ & 9,669E5 \\
\hline P08113 & Endoplasmin OS=Mus musculus GN=Hsp90b1 PE=1 SV=2 - [ENPL_MOUSE] & $0,000 E_{0}$ & $0,000 E 0$ & 1,544E6 & 4,372E7 \\
\hline P62748 & Hippocalcin-like protein 1 OS=Mus musculus GN=Hpcal1 PE=1 SV=2 - [HPCL1_MOUSE] & $0,000 E 0$ & $0,000 E 0$ & $0,000 E 0$ & $1,121 \mathrm{EE}$ \\
\hline P02088 & Hemoglobin subunit beta-1 OS=Mus musculus GN=Hbb-b1 PE=1 SV=2 - [HBB1_MOUSE] & $0,000 E 0$ & $0,000 E 0$ & $0,000 E 0$ & $1,094 \mathrm{E} 7$ \\
\hline 054781 & SRSF protein kinase 2 OS=Mus musculus GN=Srpk2 PE=1 SV=2 - [SRPK2_MOUSE] & $0,000 E_{0}$ & $0,000 E_{0}$ & $0,000 \mathrm{E}_{0}$ & $3,952 E 6$ \\
\hline P53811 & Phosphatidylinositol transfer protein beta isoform OS=Mus musculus GN=Pitpnb PE=1 SV=2 - [PIPNB_MOUSE] & $0,000 E 0$ & $0,000 E 0$ & $0,000 E 0$ & 8,31955 \\
\hline Q61286-2 & Isoform ALF1A of Transcription factor 12 OS=Mus musculus GN=Tcf12 - [HTF4_MOUSE] & $0,000 E 0$ & $0,000 E 0$ & $0,000 E 0$ & 2,336 E6 \\
\hline Q99P88 & Nuclear pore complex protein Nup155 OS=Mus musculus GN=Nup155 PE=1 SV=1 - [NU155_MOUSE] & $0,000 E 0$ & $0,000 \mathrm{E} 0$ & $0,000 \mathrm{E} 0$ & $4,020 \mathrm{E} 6$ \\
\hline Q9ESL4 & Mitogen-activated protein kinase kinase kinase MLT OS=Mus musculus GN=Zak PE=1 SV=1- [MLTK_MOUSE] & $0,000 E 0$ & $0,000 E 0$ & $0,000 E 0$ & $2,076 \mathrm{E} 6$ \\
\hline P56399 & Ubiquitin carboxyl-terminal hydrolase 5 OS=Mus musculus GN=Usp5 PE=1 SV=1 - [UBP5_MOUSE] & $0,000 E 0$ & $0,000 E 0$ & $0,000 E 0$ & $1,050 \mathrm{E} 6$ \\
\hline P47856-2 & Isoform 2 of Glutamine--fructose-6-phosphate aminotransferase [isomerizing] 1 OS=Mus musculus GN=Gfpt1 - [GFPT1_MOUSE] & $0,000 E 0$ & $0,000 E 0$ & $0,000 E 0$ & 6,250E5 \\
\hline Q8BH43 & Wiskott-Aldrich syndrome protein family member $20 \mathrm{OS}=$ Mus musculus $\mathrm{GN}=$ Wasf2 PE=1 SV=1 - [WASF2_MOUSE] & $0,000 E_{0}$ & $0,000 E_{0}$ & $0,000 \mathrm{E} 0$ & $6,262 E 6$ \\
\hline Q99NHO & Ankyrin repeat domain-containing protein 17 OS=Mus musculus GN=Ankrd17 PE=1 SV=2 - [ANR17_MOUSE] & $0,000 E_{0}$ & $0,000 E_{0}$ & $0,000 E_{0}$ & 4,666E6 \\
\hline Q9Z2F2 & 2'-5'-oligoadenylate synthase-like protein $2 \mathrm{OS}=$ Mus musculus $\mathrm{GN}=$ =asl2 PE=1 SV=2 - [OASL2_MOUSE] & $0,000 E_{0}$ & $0,000 \mathrm{E} 0$ & $0,000 E_{0}$ & $1,002 E 6$ \\
\hline P18052-2 & Isoform 2 of Receptor-type tyrosine-protein phosphatase alpha OS=Mus musculus GN=Ptpra - [PTPRA_MOUSE] & $0,000 E_{0}$ & $0,000 E_{0}$ & $0,000 E_{0}$ & $1,240 \mathrm{E} 7$ \\
\hline Q9ESW4 & Acylglycerol kinase, mitochondrial OS=Mus musculus GN=Agk PE=1 SV=1 - [AGK_MOUSE] & $0,000 E 0$ & $0,000 E_{0}$ & $0,000 E 0$ & $1,982 \mathrm{E} 6$ \\
\hline Q6P8X1 & Sorting nexin-6 OS=Mus musculus GN=Snx6 PE=1 SV=2 - [SNX6_MOUSE] & $0,000 \mathrm{E} 0$ & $0,000 \mathrm{E} 0$ & $0,000 \mathrm{E} 0$ & $2,933 \mathrm{E} 6$ \\
\hline Q8VCG3 & WD repeat-containing protein 74 OS=Mus musculus GN=Wdr74 PE=2 SV=1 - [WDR74_MOUSE] & $0,000 E_{0}$ & $0,000 E 0$ & $0,000 E 0$ & $8,721 E 5$ \\
\hline Q3TFK5-2 & Isoform 2 of $\mathrm{G}$ patch domain-containing protein 4 OS=Mus musculus GN=Gpatch4 - [GPTC4_MOUSE] & $0,000 \mathrm{E} 0$ & $0,000 \mathrm{E} 0$ & $0,000 \mathrm{E} 0$ & \begin{tabular}{l|l}
$1,561 \mathrm{E} 6$ \\
\end{tabular} \\
\hline 035344 & Importin subunit alpha-4 OS=Mus musculus GN=Kpna3 PE=1 SV=1 - [IMA4_MOUSE] & $0,000 E 0$ & $0,000 E 0$ & $0,000 E 0$ & $1,775 \mathrm{E} 6$ \\
\hline Q08093 & Calponin-2 OS=Mus musculus GN=Cnn2 PE=1 SV=1 - [CNN2_MOUSE] & $0,000 E 0$ & $0,000 E 0$ & $0,000 E 0$ & $9,081 E 5$ \\
\hline Q811D2 & Ankyrin repeat domain-containing protein 26 OS=Mus musculus $\mathrm{GN}=\mathrm{Ankrd26} \mathrm{PE}=1 \mathrm{SV}=2-[$ [ANR26_MOUSE] & $0,000 E 0$ & $0,000 E_{0}$ & $0,000 E 0$ & $3,144 E 6$ \\
\hline Q8CG47 & Structural maintenance of chromosomes protein $4 \mathrm{OS}=$ Mus musculus GN=Smc4 PE=1 SV=1 - [SMC4_MOUSE] & $0,000 \mathrm{E} 0$ & $0,000 \mathrm{E} 0$ & $0,000 \mathrm{E} 0$ & \begin{tabular}{l|l}
$1,233 \mathrm{E} 6$ \\
\end{tabular} \\
\hline Q9ROM6 & Ras-related protein Rab-9A OS=Mus musculus GN=Rab9a PE=1 SV=1 - [RAB9A_MOUSE] & $0,000 E_{0}$ & $0,000 E_{0}$ & $0,000 \mathrm{E}_{0}$ & 9,937Е5 \\
\hline P01899 & H-2 class I histocompatibility antigen, D-B alpha chain OS=Mus musculus GN=H2-D1 PE=1 SV=2 - [HA11_MOUSE] & $0,000 E 0$ & $0,000 E 0$ & $0,000 E 0$ & 3,338E6 \\
\hline Q60996-2 & Isoform 1 of Serine/threonine-protein phosphatase 2A 56 kDa regulatory subunit gamma isoform OS=Mus musculus GN=Ppp2r5c - [2A5G_MOUSE] & $0,000 E 0$ & $0,000 E 0$ & $0,000 E 0$ & $1,138 \mathrm{EE}$ \\
\hline $\begin{array}{l}\mathrm{P} 48725-3 \\
\end{array}$ & Isoform 3 of Pericentrin OS=Mus musculus GN=Pcnt - [PCNT_MOUSE] & $0,000 E 0$ & $0,000 E 0$ & $0,000 E 0$ & $4,379 \mathrm{E} 6$ \\
\hline Q8VEJ9 & Vacuolar protein sorting-associated protein 4A OS=Mus musculus $\mathrm{GN}=\mathrm{VPS4} 4 \mathrm{PE}=1 \mathrm{SV}=1$ - [VPS4A_MOUSE] & $0,000 E 0$ & $0,000 E_{0}$ & $0,000 E 0$ & 6,175E6 \\
\hline Q921F2 & TAR DNA-binding protein 43 OS=Mus musculus GN=Tardbp PE=1 SV=1 - [TADBP_MOUSE] & $0,000 E 0$ & $0,000 \mathrm{E} 0$ & $0,000 E 0$ & $1,031 \mathrm{E}$ \\
\hline 088967 & ATP-dependent zinc metalloprotease YME1L1 OS=Mus musculus GN=Yme111 PE=1 SV=1 - [YMEL1_MOUSE] & $0,000 \mathrm{E} 0$ & $0,000 E 0$ & $0,000 \mathrm{E} 0$ & 5,834E5 \\
\hline Q7TPM1 & Protein PRRC2B OS=Mus musculus GN=Prrc2b PE=1 $\mathrm{SV}=1$ - [PRC2B_MOUSE] & $0,000 E_{0}$ & $0,000 E_{0}$ & $0,000 E 0$ & $3,412 E 6$ \\
\hline Q3THK7 & GMP synthase [glutamine-hydrolyzing] OS=Mus musculus $\mathrm{GN}=\mathrm{Gmps} \mathrm{PE}=1 \mathrm{SV}=2-$ [GUAA_MOUSE] & $0,000 E 0$ & $0,000 E 0$ & $0,000 E 0$ & $2,168 E 6$ \\
\hline Q6ZPG2 & WD repeat-containing protein 90 OS=Mus musculus GN=Wdr90 PE=1 SV=2 - [WDR90_MOUSE] & $0,000 E 0$ & $0,000 E 0$ & $0,000 E 0$ & $2,192 \mathrm{E} 6$ \\
\hline Q912R2 & Sorting nexin-18 OS=Mus musculus GN=SnX18 PE=1 SV=1 - [SNX18_MOUSE] & $0,000 E 0$ & $0,000 E 0$ & $0,000 E 0$ & $5,857 \mathrm{E} 6$ \\
\hline $070585-2$ & Isoform 2 of Dystrobrevin beta OS=Mus musculus GN=Dtnb - [DTNB_MOUSE] & $0,000 E 0$ & $0,000 E 0$ & $0,000 E 0$ & $1,979 \mathrm{E} 6$ \\
\hline Q999P0 & Mitogen-activated protein kinase kinase kinase kinase 3 OS=Mus musculus GN=Map4k3 PE=1 SV=4 - [M4K3_MOUSE] & $0,000 E_{0}$ & $0,000 E_{0}$ & $0,000 E 0$ & $9,770 \mathrm{E} 5$ \\
\hline Q6A4J8-3 & Isoform 3 of Ubiquitin carboxyl-terminal hydrolase 7 OS=Mus musculus GN=USp7 - [UBP7_MOUSE] & $0,000 E 0$ & $0,000 E 0$ & $0,000 E 0$ & $5,889 \mathrm{EE}$ \\
\hline Q9CZJ2 & Heat shock $70 \mathrm{kDa}$ protein $12 \mathrm{~B}$ OS=Mus musculus GN=Hspa12b PE=1 SV=1 - [HS12B_MOUSE] & $0,000 E 0$ & $0,000 \mathrm{E} 0$ & $0,000 E 0$ & $3,971 \mathrm{E} 6$ \\
\hline Q88Z03 & Serine/threonine-protein kinase D2 OS=Mus musculus GN=Prkd2 PE=1 SV=1 - [KPCD2_MOUSE] & $0,000 E_{0}$ & $0,000 E_{0}$ & $0,000 E 0$ & $5,511 E 6$ \\
\hline Q61210-2 & Isoform 2 of Rho guanine nucleotide exchange factor 1 OS=Mus musculus GN=Arhgef1 - [ARHG1_MOUSE] & $0,000 E_{0}$ & $0,000 E_{0}$ & $0,000 \mathrm{E} 0$ & $1,986 \mathrm{E} 6$ \\
\hline Q8CG48 & Structural maintenance of chromosomes protein $2 \mathrm{OS}=$ Mus musculus GN=Smc2 PE=1 SV=2 - [SMC2_MOUSE] & $0,000 E 0$ & $0,000 E 0$ & $0,000 \mathrm{E} 0$ & 3,193E6 \\
\hline Q8C151 & PDZ and LIM domain protein 5 OS=Mus musculus GN=Pdlim5 PE=1 SV=4 - [PDLI5_MOUSE] & $0,000 E_{0}$ & $0,000 E_{0}$ & $0,000 E 0$ & $1,462 E 6$ \\
\hline P49615 & Cyclin-dependent-like kinase 5 OS=Mus musculus GN=Cdk5 PE=1 SV=1-[CDK5_MOUSE] & $0,000 E 0$ & $0,000 E 0$ & $0,000 \mathrm{E} 0$ & $2,128 \mathrm{E} 7$ \\
\hline Q8KOV4 & CCR4-NOT transcription complex subunit 3 OS=Mus musculus GN=Cnot3 PE=1 SV=1- [CNOT3_MOUSE] & $0,000 E 0$ & $0,000 E_{0}$ & $0,000 E 0$ & $2,547 \mathrm{E}$ \\
\hline P43406 & Integrin alpha-V OS=Mus musculus GN=Itgav PE=1 SV=2 - [TTAV_MOUSE] & $0,000 E 0$ & $0,000 E_{0}$ & $0,000 \mathrm{E} 0$ & $4,718 \mathrm{E} 6$ \\
\hline E9PVX6 & Proliferation marker protein Ki-67 OS=Mus musculus GN=Mki67 PE=1 SV=1 - [KI67_MOUSE] & $0,000 E_{0}$ & $0,000 E_{0}$ & $0,000 \mathrm{E}_{0}$ & $1,850 \mathrm{E} 6$ \\
\hline Q9DCN2-2 & Isoform 2 of NADH-cytochrome b5 reductase 3 OS=Mus musculus GN=Cyb5r3 - [NBSR3_MOUSE] & $0,000 E 0$ & $0,000 E 0$ & $0,000 E 0$ & $4,026 E 6$ \\
\hline P09103 & Protein disulfide-isomerase $\mathrm{OS}=$ Mus musculus $\mathrm{GN}=\mathrm{P} 4 \mathrm{hb} \mathrm{PE}=1 \mathrm{SV}=2-[$ PDIA1_MOUSE] & $0,000 E 0$ & $0,000 E 0$ & $0,000 E 0$ & 4,29766 \\
\hline P30416 & Peptidyl-prolyl cis-trans isomerase FKBP4 OS=Mus musculus GN=Fkbp4 PE=1 SV=5 - [FKBP4_MOUSE] & $0,000 \mathrm{E} 0$ & $0,000 E 0$ & $0,000 E 0$ & $7,166 \mathrm{E} 5$ \\
\hline Q911T7 & YTH domain-containing family protein $2 \mathrm{OS}=$ Mus musculus $\mathrm{GN}=\mathrm{Ythdf2} P \mathrm{PE}=1 \mathrm{SV}=1$ - [YTHD2_MOUSE] & $0,000 E_{0}$ & $0,000 E_{0}$ & $5,369 \mathrm{E} 6$ & $5,946 \mathrm{E} 6$ \\
\hline Q8BVYY & Ribosomal L1 domain-containing protein 1 OS=Mus musculus GN=RsI1d1 PE=1 SV=1 - [RL1D1_MOUSE] & $0,000 E 0$ & $0,000 E 0$ & $0,000 E 0$ & $1,323 E 6$ \\
\hline Q90883 & Splicing factor U2AF $35 \mathrm{kDa}$ subunit OS=Mus musculus GN=U2af1 PE=1 SV=4 - [U2AF1_MOUSE] & $0,000 E 0$ & $0,000 E 0$ & $0,000 E 0$ & $5,700 E 6$ \\
\hline A2RTL5 & Arginine/serine-rich coiled-coil protein 2 OS=Mus musculus GN=Rsrc2 PE=2 SV=1 - [RSRC2_MOUSE] & $0,000 E 0$ & $0,000 E 0$ & $0,000 E 0$ & $2,971 E 6$ \\
\hline Q9CXW2 & $28 \mathrm{~S}$ ribosomal protein S22, mitochondrial OS=Mus musculus GN=Mrps22 PE=1 SV=1 - [RT22_MOUSE] & $0,000 E_{0}$ & $0,000 E_{0}$ & $0,000 E 0$ & 3,939E6 \\
\hline 070305-2 & Isoform 2 of Ataxin-2 OS=Mus musculus GN=Atxn2 - [ATX2_MOUSE] & $0,000 E 0$ & $0,000 E 0$ & $0,000 E 0$ & $5,520 \mathrm{E} 6$ \\
\hline
\end{tabular}




\begin{tabular}{|c|c|c|c|c|c|}
\hline Q9ERG2 & Striatin-3 OS=Mus musculus GN=Strn3 PE=1 SV=1 - [STRN3_MOUSE] & $0,000 \mathrm{E} 0$ & $0,000 \mathrm{E} 0$ & $0,000 E 0$ & 1,089E6 \\
\hline Q8BWB6 & Metalloreductase STEAP2 OS=Mus musculus GN=Steap2 PE=1 SV=1 - [STEA2_MOUSE] & $0,000 \mathrm{E} 0$ & $0,000 \mathrm{E} 0$ & $0,000 E 0$ & $3,532 \mathrm{E} 6$ \\
\hline P70248 & Unconventional myosin-If OS=Mus musculus GN=Myo1f PE=1 SV=1 - [MYO1F_MOUSE] & $0,000 E 0$ & $0,000 \mathrm{E} 0$ & $2,601 E 6$ & $1,353 \mathrm{E} 8$ \\
\hline $054988-2$ & Isoform 2 of STE20-like serine/threonine-protein kinase OS=Mus musculus GN=Slk - [SLK_MOUSE] & $0,000 \mathrm{E} 0$ & $0,000 \mathrm{E} 0$ & $0,000 \mathrm{E} 0$ & $2,328 E 6$ \\
\hline Q7TN58 & Transmembrane channel-like protein 8 OS=Mus musculus GN=Tmc8 PE=1 SV=2 - [TMC8_MOUSE] & $0,000 E 0$ & $0,000 E 0$ & $0,000 E 0$ & 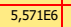 \\
\hline Q882X4 & Splicing factor 45 OS=Mus musculus GN=Rbm17 PE=1 SV=1 - [SPF45_MOUSE] & $0,000 \mathrm{E} 0$ & $0,000 \mathrm{E} 0$ & $0,000 E 0$ & $1,023 E 6$ \\
\hline Q9DCU6 & $39 \mathrm{~S}$ ribosomal protein L4, mitochondrial OS=Mus musculus GN=Mrpl4 PE=1 SV=1 - [RM04_MOUSE] & $0,000 E_{0}$ & $0,000 E 0$ & $0,0000_{0}$ & $1,707 \mathrm{E} 6$ \\
\hline P60766 & Cell division control protein 42 homolog OS=Mus musculus GN=Cdc42 PE=1 $\mathrm{SV}=2$ - [CDC42_MOUSE] & $0,000 \mathrm{E} 0$ & $0,000 \mathrm{E} 0$ & $0,000 E 0$ & $3,260 \mathrm{E} 6$ \\
\hline Q921M3 & Splicing factor $3 \mathrm{~B}$ subunit $3 \mathrm{OS}=$ Mus musculus $\mathrm{GN}=\mathrm{S} F 3 \mathrm{b3} \mathrm{PE}=1 \mathrm{SV}=1$ - [SF3B3_MOUSE] & $0,000 \mathrm{E} 0$ & $0,000 E 0$ & $0,000 E 0$ & $2,382 E 6$ \\
\hline Q8C5Q4 & G-rich sequence factor 1 OS=Mus musculus GN=Grsf1 PE=1 SV=2 - [GRSF1_MOUSE] & $0,000 \mathrm{E} 0$ & $0,000 \mathrm{E} 0$ & $0,000 E 0$ & 1,194E6 \\
\hline LON7N1 & Kinesin-like protein KIF14 OS=Mus musculus GN=Kif14 PE=1 SV=1 - [KIF14_MOUSE] & $0,000 \mathrm{E} 0$ & $0,000 E 0$ & $0,000 E 0$ & $2,803 E 6$ \\
\hline Q8CG79 & Apoptosis-stimulating of p53 protein 2 OS=Mus musculus GN=Tp53bp2 PE=1 SV=3 - [ASPP2_MOUSE] & 0,000 E & $0,000 \mathrm{E} 0$ & $0,000 E 0$ & \begin{tabular}{l|l}
$4,976 \mathrm{E} 6$ \\
\end{tabular} \\
\hline Q9D014 & ADP-ribosylation factor-like protein $2 \mathrm{OS}=$ Mus musculus GN=Arl2 PE=1 SV=1 - [ARLL_MOUSE] & $0,000 \mathrm{E} 0$ & $0,000 E 0$ & $0,000 E 0$ & $3,752 \mathrm{E} 6$ \\
\hline Q8CB44 & GRAM domain-containing protein 4 OS=Mus musculus GN=Gramd4 PE=1 SV=1 - [GRAM4_MOUSE] & $0,000 \mathrm{E}_{0}$ & $0,000 \mathrm{E} 0$ & $0,000 E 0$ & $1,775 E 6$ \\
\hline Q9DBR4 & Amyloid beta A4 precursor protein-binding family B member $2 \mathrm{OS}=$ Mus musculus $\mathrm{GN}=\mathrm{Apbb2} \mathrm{PE}=1 \mathrm{SV}=2$ - [APBB2_MOUSE] & $0,000 \mathrm{E} 0$ & $0,000 \mathrm{E} 0$ & $0,000 \mathrm{E} 0$ & $1,942 E 6$ \\
\hline P13864 & DNA (cytosine-5)-methyltransferase 1 OS=Mus musculus GN=Dnmt1 PE=1 SV=5 - [DNMT1_MOUSE] & $0,000 E 0$ & $0,000 \mathrm{E} 0$ & $0,000 E 0$ & $2,100 E 6$ \\
\hline P58660 & Caspase recruitment domain-containing protein 10 OS=Mus musculus GN=Card10 PE=1 SV=1 - [CAR10_MOUSE] & $0,000 \mathrm{E} 0$ & $0,000 \mathrm{E} 0$ & $0,000 E 0$ & $1,067 \mathrm{E}$ \\
\hline Q9ZOH3-2 & Isoform B of SWI/SNF-related matrix-associated actin-dependent regulator of chromatin subfamily B member 1 OS=Mus musculus GN=Smarcb1 - [SNF5_MOUSE] & $0,000 E_{0}$ & $0,000 E 0$ & $0,000 E 0$ & 1,930E6 \\
\hline Q9CQ10 & Charged multivesicular body protein 3 OS=Mus musculus GN=Chmp3 PE=1 SV=3 - [CHMP3_MOUSE] & $0,000 E 0$ & $0,000 E 0$ & $0,000 E 0$ & 9,984E6 \\
\hline Q9R0X4 & Acyl-coenzyme A thioesterase 9 , mitochondrial OS=Mus musculus GN=Acot9 PE=1 $\mathrm{SV}=1$ - [ACOT9_MOUSE] & $0,000 \mathrm{E} 0$ & $0,000 \mathrm{E} 0$ & $0,000 E 0$ & $2,974 E 6$ \\
\hline Q7TNP2 & Serine/threonine-protein phosphatase $2 \mathrm{~A} 65 \mathrm{kD}$ regulatory subunit A beta isoform OS=Mus musculus GN=Ppp2r1b PE=1 SV=2 - [2AAB_MOUSE] & $0,000 \mathrm{E} 0$ & $0,000 \mathrm{E} 0$ & $0,000 E 0$ & $1,239 \mathrm{E} 7$ \\
\hline Q64322 & Neural proliferation differentiation and control protein 1 OS=Mus musculus GN=Npdc1 PE=2 SV=2 - [NPDC1_MOUSE] & $0,000 E_{0}$ & $0,000 E_{0}$ & $0,000 \mathrm{E}_{0}$ & $4,126 \mathrm{E} 6$ \\
\hline 035379 & Multidrug resistance-associated protein 1 OS=Mus musculus GN=Abcc1 PE=1 SV=1 - [MRP1_MOUSE] & $0,000 E_{0}$ & $0,000 E_{0}$ & $0,0000_{0}$ & $2,495 \mathrm{EE}$ \\
\hline Q9CQC9 & GTP-binding protein SAR1b OS=Mus musculus GN=Sar1b PE=1 SV=1 - [SAR1B_MOUSE] & $0,000 E_{0}$ & $0,000 \mathrm{E} 0$ & $0,000 E 0$ & $5,084 E 6$ \\
\hline Q9WUN2 & Serine/threonine-protein kinase TBK1 OS=Mus musculus GN=Tbk1 PE=1 SV=1 - [TBK1_MOUSE] & $0,000 \mathrm{E} 0$ & $0,000 E 0$ & $0,000 E 0$ & $1,266 \mathrm{E} 6$ \\
\hline Q9Q776 & Vesicle-associated membrane protein-associated protein B OS=Mus musculus GN=Vapb PE=1 SV=3 - [VAPB_MOUSE] & $0,000 \mathrm{E} 0$ & $0,000 \mathrm{E} 0$ & $0,000 E 0$ & $2,272 E 6$ \\
\hline P70349 & Histidine triad nucleotide-binding protein 1 OS=Mus musculus GN=Hint1 PE=1 SV=3 - [HINT1_MOUSE] & $0,000 \mathrm{E} 0$ & $0,000 \mathrm{E} 0$ & $0,000 E 0$ & $1,951 E 7$ \\
\hline Q61292 & Laminin subunit beta-2 $\mathrm{OS}=$ Mus musculus $\mathrm{GN}=\mathrm{Lamb2} \mathrm{PE}=1 \mathrm{SV}=2$ - [LAMB2_MOUSE] & $0,000 \mathrm{E} 0$ & $0,000 \mathrm{E} 0$ & $0,000 E 0$ & $1,502 E 6$ \\
\hline Q8BMS1 & Trifunctional enzyme subunit alpha, mitochondrial OS=Mus musculus $\mathrm{GN}=\mathrm{Hadha} P \mathrm{PE}=1 \mathrm{SV}=1$ - [ECHA_MOUSE] & $0,000 \mathrm{E} 0$ & $0,000 \mathrm{E} 0$ & $0,000 E 0$ & 5,19766 \\
\hline P10493 & Nidogen-1 OS=Mus musculus GN=Nid1 PE=1 SV=2 - [NID1_MOUSE] & $0,000 E 0$ & $0,000 E 0$ & $0,000 E 0$ & $2,374 E 6$ \\
\hline Q9CZX0 & Elongator complex protein 3 OS=Mus musculus $\mathrm{GN}=E \mathrm{El} 3 \mathrm{PE}=1 \mathrm{SV}=1$ - [ELP3_MOUSE] & $0,000 E 0$ & $0,000 E 0$ & $0,000 E 0$ & $9,901 E 5$ \\
\hline Q6PFQ7 & Ras GTPase-activating protein 4 OS=Mus musculus GN=Rasa4 PE=1 SV=1 - [RASL2_MOUSE] & $0,000 \mathrm{E} 0$ & $0,000 \mathrm{E} 0$ & $0,000 E 0$ & $1,237 E 6$ \\
\hline Q8BG02 & Serine/threonine-protein phosphatase 2A $55 \mathrm{kDa}$ regulatory subunit B gamma isoform OS=Mus musculus GN=Ppp2r2c PE=1 SV=1 - [2ABG_MOUSE] & $0,000 E 0$ & $0,000 \mathrm{E} 0$ & $0,000 E 0$ & 2,757766 \\
\hline Q5XJY5 & Coatomer subunit delta OS=Mus musculus GN=Arcn1 PE=1 SV=2 - [COPD_MOUSE] & $0,000 E_{0}$ & $0,000 \mathrm{E} 0$ & $0,000 \mathrm{E}_{0}$ & $1,114 \mathrm{E} 6$ \\
\hline P54761 & Ephrin type-B receptor 4 OS=Mus musculus GN=Ephb4 PE=1 SV=2 - [EPHB4_MOUSE] & $0,000 E 0$ & $0,000 E 0$ & $0,000 E_{0}$ & $\begin{array}{ll}4,637 E 6 \\
\end{array}$ \\
\hline Q9IIF7 & Coatomer subunit beta $\mathrm{OS}=$ Mus musculus $\mathrm{GN}=\mathrm{Copb} 1 \mathrm{PE}=1 \mathrm{SV}=1-$ [COPB_MOUSE] & $0,000 \mathrm{E} 0$ & $0,000 \mathrm{E} 0$ & $0,000 E 0$ & 4,996E5 \\
\hline Q8BJU2 & Tetraspanin-9 OS=Mus musculus GN=Tspan9 PE=1 SV=1 - [TSN9_MOUSE] & $0,000 \mathrm{E} 0$ & $0,000 \mathrm{E} 0$ & $0,000 E 0$ & 2,053E6 \\
\hline Q57114 & Tyrosine-protein kinase SgK223 OS=Mus musculus GN=Sgk223 PE=1 SV=2 - [SG223_MOUSE] & $0,000 E 0$ & $0,000 E 0$ & $0,0000_{0}$ & $1,445 E 6$ \\
\hline Q5SFM8 & RNA-binding protein 27 OS=Mus musculus GN=Rbm27 PE=1 SV=3 - [RBM27_MOUSE] & $0,000 \mathrm{E} 0$ & $0,000 \mathrm{E} 0$ & $0,000 E 0$ & 4,092E6 \\
\hline Q8BRN9 & Coiled-coil and C2 domain-containing protein $1 \mathrm{~B} 0 \mathrm{OS}=$ Mus musculus $\mathrm{GN}=\mathrm{C} C 2 \mathrm{~d} 1 \mathrm{~b} P E=1 \mathrm{SV}=1-$ [C2D1B_MOUSE] & $0,000 \mathrm{E} 0$ & $0,000 \mathrm{E} 0$ & $0,000 E 0$ & $2,454 E 6$ \\
\hline Q8BX17 & Gem-associated protein 5 OS=Mus musculus GN=Gemin5 PE=1 SV=2 - [GEMI5_MOUSE] & $0,000 \mathrm{E} 0$ & $0,000 \mathrm{E} 0$ & $0,000 E 0$ & $2,525 \mathrm{E} 6$ \\
\hline Q61545 & RNA-binding protein EWS OS=Mus musculus GN=Ewsr1 PE=1 $1 \mathrm{SV}=2$ - [EWS_MOUSE] & 0,000E0 & 0,000E0 & $0,000 \mathrm{E} 0$ & $1,766 \mathrm{E} 7$ \\
\hline Q9CZD3 & Glycine--tRNA ligase OS=Mus musculus GN=Gars PE=1 SV=1 - [SYG_MOUSE] & $0,000 \mathrm{E} 0$ & $0,000 E 0$ & $0,000 E 0$ & $2,348 E 6$ \\
\hline P10922 & Histone H1.0 OS=Mus musculus GN=H1fo PE=2 SV=4 - [H10_MOUSE] & $0,000 \mathrm{E} 0$ & $0,000 \mathrm{E} 0$ & $0,000 E 0$ & $6,867 \mathrm{E}$ \\
\hline Q8R550-5 & Isoform 5 of SH3 domain-containing kinase-binding protein 1 OS=Mus musculus GN=Sh3kbp1 - [SH3K1_MOUSE] & $0,000 E_{0}$ & $0,000 \mathrm{E} 0$ & $0,0000_{0}$ & $1,205 E 6$ \\
\hline Q91YE6 & Importin-9 OS=Mus musculus GN=Ipo9 PE=1 SV=3 - [IPO9_MOUSE] & $0,000 E 0$ & $0,000 E 0$ & $0,000 E 0$ & 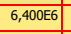 \\
\hline 035149 & Zinc transporter 4 OS=Mus musculus GN=Slc30a4 PE=1 SV=2 - [ZNT4_MOUSE] & $0,000 E 0$ & $0,000 E 0$ & $0,000 E 0$ & $3,931 E 6$ \\
\hline Q3V1V3 & ESF1 homolog OS=Mus musculus GN=Esf1 PE=1 SV=1 - [ESF1_MOUSE] & $0,000 E 0$ & $0,000 \mathrm{E} 0$ & $0,000 E 0$ & $5,764 E 6$ \\
\hline Q6URW6-2 & Isoform 2 of Myosin-14 OS=Mus musculus GN=Myh14 - [MYH14_MOUSE] & $2,336 \mathrm{E} 8$ & $0,000 E 0$ & $5,102 E 7$ & $2,921 \mathrm{Eg}$ \\
\hline Q9R128 & Vinexin OS=Mus musculus GN=Sorbs3 PE=1 SV=1 - [VINEX_MOUSE] & $0,000 E 0$ & $0,000 \mathrm{E} 0$ & $0,000 \mathrm{E}_{0}$ & $8,258 E 6$ \\
\hline P70207 & Plexin-A2 OS=Mus musculus GN=Plxna2 PE=1 SV=2 - [PLXA2_MOUSE] & $0,000 \mathrm{E} 0$ & $0,000 \mathrm{E} 0$ & $0,000 E 0$ & 6,187E6 \\
\hline Q32P12-2 & Isoform 2 of Uncharacterized protein C17orf53 homolog OS=Mus musculus - [CQ053_MOUSE] & $0,000 \mathrm{E} 0$ & $0,000 \mathrm{E} 0$ & $0,000 E 0$ & $2,390 \mathrm{E} 6$ \\
\hline Q9CXF4 & TBC1 domain family member 15 OS=Mus musculus GN=Tbc1d15 PE=1 SV=1 - [TBC15_MOUSE] & $0,000 \mathrm{E} 0$ & $0,000 \mathrm{E} 0$ & $0,000 E 0$ & $1,078 E 6$ \\
\hline 088554 & Poly [ADP-ribose] polymerase 2 OS=Mus musculus GN=Parp2 PE=1 $\mathrm{SV}=3$ - [PARP2_MOUSE] & $0,000 E 0$ & $0,000 E 0$ & $0,000 E 0$ & 9,644E5 \\
\hline Q99LU0 & Charged multivesicular body protein 1b-1 OS=Mus musculus GN=Chmp1b1 PE=1 SV=1 - [CH1B1_MOUSE] & $0,000 \mathrm{E} 0$ & $0,000 \mathrm{E} 0$ & $0,000 E 0$ & $1,233 \mathrm{E} 7$ \\
\hline Q9CZX5 & PIN2/TERF1-interacting telomerase inhibitor 1 OS=Mus musculus GN=Pinx1 PE=1 SV=2 - [PINX1_MOUSE] & $0,000 \mathrm{E} 0$ & $0,000 E 0$ & $0,000 E 0$ & $1,479 E 6$ \\
\hline Q8R326-2 & Isoform 2 of Paraspeckle component 1 OS=Mus musculus GN=Pspc1 - [PSPC1_MOUSE] & $0,000 \mathrm{E} 0$ & $0,000 \mathrm{E} 0$ & $0,000 E 0$ & $7,780 \mathrm{E} 6$ \\
\hline Q9QZM4 & Tumor necrosis factor receptor superfamily member 10B OS=Mus musculus $\mathrm{GN}=$ Tnfrsf10b PE=2 SV=3 - [TR10B_MOUSE] & $0,000 E 0$ & $0,000 E 0$ & $0,000 E 0$ & $2,374 E 6$ \\
\hline Q8BWG8-2 & Isoform 1B of Beta-arrestin-1 OS=Mus musculus GN=Arrb1 - [ARRB1_MOUSE] & $0,000 \mathrm{E}_{0}$ & $0,000 \mathrm{E} 0$ & $0,000 E 0$ & $2,430 \mathrm{E} 6$ \\
\hline Q9JLB0-2 & Isoform Alpha of MAGUK p55 subfamily member 6 OS=Mus musculus GN=Mpp6 - [MPP6_MOUSE] & $0,000 \mathrm{E} 0$ & $0,000 \mathrm{E} 0$ & $0,000 \mathrm{E} 0$ & $1,225 \mathrm{E} 6$ \\
\hline Q80XI6 & Mitogen-activated protein kinase kinase kinase 11 OS=Mus musculus GN=Map3k11 PE=1 SV=1 - [M3K11_MOUSE] & $0,000 E 0$ & $0,000 E 0$ & $0,000 E 0$ & $4,111 \mathrm{ES}$ \\
\hline Q902Y4-2 & Isoform 2 of Mixed lineage kinase domain-like protein OS=Mus musculus GN=MIk1 - [MLKL_MOUSE] & $0,000 \mathrm{E} 0$ & $0,000 \mathrm{E} 0$ & $0,000 E 0$ & $1,071 \mathrm{E} 6$ \\
\hline P12894 & Probable Pol polyprotein OS=Mouse intracisternal a-particle IL3 PE=3 SV=1 - [POL_IPMAI] & $0,000 E_{0}$ & $0,000 E 0$ & $0,000 E 0$ & 1,981E6 \\
\hline Q8BHF7 & CDP-diacylglycerol--glycerol-3-phosphate 3-phosphatidyltransferase, mitochondrial OS=Mus musculus GN=Pgs1 PE=1 SV=1 - [PGPS1_MOUSE] & $0,000 E 0$ & $0,000 E 0$ & $0,000 E 0$ & 3,37466 \\
\hline Q6PB44-2 & Isoform 2 of Tyrosine-protein phosphatase non-receptor type 23 OS=Mus musculus GN=Ptpn23 - [PTN23_MOUSE] & $0,000 \mathrm{E} 0$ & $0,000 \mathrm{E} 0$ & $0,000 E 0$ & $2,543 \mathrm{E} 6$ \\
\hline 035368 & Interferon-activable protein 203 OS=Mus musculus GN=Ifr203 PE=1 SV=1 - [FFI3_MOUSE] & $0,000 \mathrm{E} 0$ & $0,000 \mathrm{E} 0$ & $0,000 E 0$ & 3,036E6 \\
\hline Q8K019-3 & Isoform 3 of Bcl-2-associated transcription factor 1 OS=Mus musculus GN=Bclaf1 - [BCLF1_MOUSE] & $0,000 E_{0}$ & $0,000 E_{0}$ & $0,0000_{0}$ & $6,968 E 5$ \\
\hline Q8BG95 & Protein phosphatase 1 regulatory subunit 12B OS=Mus musculus GN=Ppp1112b PE=1SV=2 - [MYPT2_MOUSE] & 0,000E0 & $0,000 \mathrm{E}$ & $0,000 E 0$ & 6,992E7 \\
\hline 009172 & Glutamate--cysteine ligase regulatory subunit OS=Mus musculus $\mathrm{GN}=\mathrm{Gclm} P \mathrm{PE}=1 \mathrm{SV}=1$ - [GSHO_MOUSE] & $0,000 \mathrm{E} 0$ & $0,000 \mathrm{E} 0$ & $0,000 E 0$ & $2,737 \mathrm{E}$ \\
\hline Q9CX00 & IST1 homolog OS=Mus musculus GN=Ist1 PE=1 SV=1 - [IST1_MOUSE] & $0,000 \mathrm{E} 0$ & $0,000 \mathrm{E} 0$ & $0,000 E 0$ & 4,093E6 \\
\hline 009106 & Histone deacetylase $1 \mathrm{OS}=$ Mus musculus GN=Hdac1 PE=1 SV=1 - [HDAC1_MOUSE] & $0,000 \mathrm{E} 0$ & $0,000 \mathrm{E} 0$ & $0,000 E 0$ & $4,690 \mathrm{E} 6$ \\
\hline Q8BRT1-5 & Isoform 2 of CLIP-associating protein 2 OS=Mus musculus GN=Clasp2 - [CLAP2_MOUSE] & 0,000E0 & 0,000E0 & $0,000 \mathrm{E} 0$ & $4,562 E 5$ \\
\hline Q80UY2-2 & Isoform 2 of E3 ubiquitin-protein ligase KCMF1 OS=Mus musculus GN=Kcmf1 - [KCMF1_MOUSE] & $0,000 E 0$ & $0,000 \mathrm{E} 0$ & $0,000 \mathrm{E} 0$ & $5,965 E 6$ \\
\hline P51912 & Neutral amino acid transporter $B(0)$ OS=Mus musculus GN=Slc1a5 PE=1 SV=2 - [AAAT_MOUSE] & $0,000 \mathrm{E} 0$ & $0,000 \mathrm{E} 0$ & $0,000 E 0$ & $2,869 \mathrm{E} 6$ \\
\hline P46460 & Vesicle-fusing ATPase OS=Mus musculus GN=Nsf PE=1 SV=2 - [NSF_MOUSE] & $0,000 E 0$ & $0,000 E 0$ & $0,000 E 0$ & $1,126 \mathrm{E}$ \\
\hline Q9DOR4 & Probable ATP-dependent RNA helicase DDX56 OS=Mus musculus GN=Dd×56 PE=2 SV=1 - [DDX56_MOUSE] & $0,000 \mathrm{E} 0$ & $0,000 \mathrm{E} 0$ & $0,000 E 0$ & $2,047 E 6$ \\
\hline A2AGTS-2 & Isoform 2 of Cytoskeleton-associated protein 5 OS=Mus musculus GN=Ckap5 - [CKAP5_MOUSE] & $0,000 \mathrm{E} 0$ & $0,000 \mathrm{E} 0$ & $0,000 E 0$ & 3,313E6 \\
\hline P70288 & Histone deacetylase 2 OS=Mus musculus GN=Hdac2 PE=1 SV=1 - [HDAC2_MOUSE] & $0,000 \mathrm{E} 0$ & $0,000 \mathrm{E} 0$ & $0,000 E 0$ & $2,860 \mathrm{E} 6$ \\
\hline 035963 & Ras-related protein Rab-33B OS=Mus musculus GN=Rab33b PE=1 SV=1 - [RB33B_MOUSE] & $0,000 E_{0}$ & $0,000 E 0$ & $3,456 \mathrm{E}$ & $1,027 \mathrm{E} 8$ \\
\hline P23475 & X-ray repair cross-complementing protein 6 OS=Mus musculus GN=Xrcc6 PE=1 SV=5 - [XRCC6_MOUSE] & $0,000 E 0$ & $0,000 E 0$ & $0,000 E 0$ & $1,892 E 6$ \\
\hline Q8VC03 & Echinoderm microtubule-associated protein-like 3 OS=Mus musculus $\mathrm{GN}=\mathrm{Em} \mid 3 \mathrm{PE}=1 \mathrm{SV}=1$ - [EMAL3_MOUSE] & $0,000 \mathrm{E} 0$ & $0,000 \mathrm{E} 0$ & $0,000 E 0$ & $2,787 \mathrm{E}$ \\
\hline Q3UDE2 & Tubulin--tyrosine ligase-like protein 12 OS=Mus musculus GN=Tt|12 PE=1 SV=1 - [TTL12_MOUSE] & $0,000 \mathrm{E} 0$ & $0,000 \mathrm{E} 0$ & $0,000 E 0$ & $7,815 E 5$ \\
\hline Q6ZPR6 & Inhibitor of Bruton tyrosine kinase OS=Mus musculus GN=Ibtk PE=1 SV=3 - [IBTK_MOUSE] & $0,000 \mathrm{E} 0$ & $0,000 \mathrm{E} 0$ & $0,000 E 0$ & $1,539 \mathrm{E} 6$ \\
\hline Q9CUN6 & E3 ubiquitin-protein ligase SMURF1 OS=Mus musculus GN=Smurf1 PE=1 SV=2 - [SMUF1_MOUSE] & $0,000 \mathrm{E} 0$ & $0,000 \mathrm{E} 0$ & $0,000 E 0$ & $9,328 \mathrm{E} 6$ \\
\hline P39876 & Metalloproteinase inhibitor 3 OS=Mus musculus GN=Timp3 PE=1 SV=1 - [TIMP3_MOUSE] & $0,000 \mathrm{E} 0$ & $0,000 \mathrm{E} 0$ & $0,000 E 0$ & 4,363E6 \\
\hline P34152-3 & Isoform 3 of Focal adhesion kinase $10 \mathrm{OS}=$ Mus musculus GN=Ptk2 - [FAK1_MOUSE] & $0,000 \mathrm{E} 0$ & $0,000 \mathrm{E} 0$ & $0,000 E 0$ & $1,661 \mathrm{E} 6$ \\
\hline P40201 & Chromodomain-helicase-DNA-binding protein 1 OS=Mus musculus GN=Chd1 PE=1 SV=3 - [CHD1_MOUSE] & $0,000 \mathrm{E} 0$ & $0,000 E 0$ & $0,000 E 0$ & 3,349E6 \\
\hline P97440 & Histone RNA hairpin-binding protein OS=Mus musculus GN=Slbp PE=1 SV=1 - [SLBP_MOUSE] & $0,000 \mathrm{E} 0$ & $0,000 \mathrm{E} 0$ & $0,000 E 0$ & $4,769 \mathrm{E} 6$ \\
\hline Q8VCL2 & Protein $\mathrm{SCO} 2$ homolog, mitochondrial OS=Mus musculus GN=SCO2 PE=1 SV=1- [SCO2_MOUSE] & $0,000 \mathrm{E} 0$ & $0,000 \mathrm{E} 0$ & $0,000 E 0$ & $7,012 E 5$ \\
\hline Q6Q477 & Plasma membrane calcium-transporting ATPase 4 OS=Mus musculus GN=Atp2b4 PE=1 SV=1 - [AT2B4_MOUSE] & $0,000 E_{0}$ & $0,000 \mathrm{E} 0$ & $0,0000_{0}$ & $8,105 E 6$ \\
\hline Q9ER72 & Cysteine--tRNA ligase, cytoplasmic OS=Mus musculus GN=Cars PE=1 SV=2 - [SYCC_MOUSE] & $0,000 \mathrm{E} 0$ & $0,000 \mathrm{E} 0$ & $0,000 E 0$ & $1,909 \mathrm{E} 6$ \\
\hline Q5SWD9 & Pre-rRNA-processing protein TSR1 homolog OS=Mus musculus GN=Tsr1 PE=1 SV=1 - [TSR1_MOUSE] & $0,000 \mathrm{E} 0$ & $0,000 \mathrm{E} 0$ & $0,000 E 0$ & $3,056 \mathrm{E} 6$ \\
\hline PODOV2 & Interferon-activable protein 204 OS=Mus musculus & $0,000 E 0$ & $0,000 \mathrm{E} 0$ & $0,000 E 0$ & $2,361 \mathrm{E} 6$ \\
\hline
\end{tabular}




\begin{tabular}{|c|c|c|c|c|c|}
\hline Q9ERK4 & Exportin-2 OS=Mus musculus GN=Cse11 PE=1 SV=1 - [XPO2__MOUSE] & $0,000 \mathrm{EO}^{-}$ & $0,000 \mathrm{E} 0$ & $0,000 E_{0}$ & $2,980 E 6$ \\
\hline P11365 & Retrovirus-related Gag polyprotein OS=Mouse intracisternal a-particle MIA14 GN=gag PE=4 SV=2 - [GAG_IPMA] & $0,000 E 0$ & $0,000 E 0$ & $0,0000_{0}$ & $1,226 \mathrm{E} 7$ \\
\hline Q80X73 & Protein pelota homolog OS=Mus musculus GN=Pelo PE=1 $\mathrm{SV}=3$ - [PELO_MOUSE] & $0,000 E 0$ & $0,000 E 0$ & $0,000 E 0$ & $4,291 \mathrm{E} 6$ \\
\hline Q7Tा37 & Elongator complex protein 1 OS=Mus musculus GN=Ikbkap PE=1 SV=2 - [ELP1_MOUSE] & $0,000 \mathrm{E} 0$ & $0,000 \mathrm{E} 0$ & $0,000 E 0$ & $2,022 E 6$ \\
\hline Q6PIX5 & Inactive rhomboid protein 1 OS=Mus musculus GN=Rhbdf1 PE=1 SV=2 - [RHDF1_MOUSE] & $0,000 \mathrm{E} 0$ & $0,000 \mathrm{E} 0$ & $0,000 E 0$ & $3,128 \mathrm{E} 6$ \\
\hline Q62191 & E3 ubiquitin-protein ligase TRIM21 OS=Mus musculus GN=Trim21 PE=1 SV=1 - [RO52_MOUSE] & $0,000 \mathrm{E} 0$ & $0,000 \mathrm{E} 0$ & 1,073E6 & $1,027 \mathrm{~F}$ \\
\hline P35123 & Ubiquitin carboxyl-terminal hydrolase 4 OS=Mus musculus GN=Usp4 PE=1 SV=3 - [UBP4_MOUSE] & $0,000 E_{0}$ & $0,000 E 0$ & $0,000 E 0$ & $3,696 \mathrm{E} 5$ \\
\hline Q6PGL7-2 & Isoform 2 of WASH complex subunit 2 OS=Mus musculus GN=Washc2 - [WASC2_MOUSE] & $0,000 \mathrm{E} 0$ & $0,000 E 0$ & $0,000 \mathrm{E} 0$ & $1,493 E 6$ \\
\hline Q99P72 & Reticulon-4 OS=Mus musculus GN=Rtn4 PE=1 SV=2 - [RTN4_MOUSE] & $0,000 E 0$ & $0,000 E 0$ & $0,000 E_{0}$ & 6,56956 \\
\hline 008919 & Numb-like protein OS=Mus musculus GN=Numbl PE=1 SV=3 - [NUMBL_MOUSE] & $0,000 \mathrm{E} 0$ & $0,000 \mathrm{E} 0$ & $0,000 \mathrm{E}_{0}$ & $7,608 \mathrm{E} 6$ \\
\hline 008582 & GTP-binding protein 1 OS=Mus musculus GN=Gtpbp1 PE=1 SV=2 - [GTPB1_MOUSE] & $0,000 E_{0}$ & $0,000 E 0$ & $0,000 E_{0}$ & $1,521 \mathrm{EE}$ \\
\hline Q8R3B1 & 1-phosphatidylinositol 4,5-bisphosphate phosphodiesterase delta-1 $1 \mathrm{OS}=$ Mus musculus GN=Plcd1 PE=1SV=2 - [PLCD1_MOUSE] & $0,000 \mathrm{E} 0$ & $0,000 \mathrm{E} 0$ & $0,000 \mathrm{E} 0$ & $1,428 \mathrm{EE} 6$ \\
\hline Q02858 & Angiopoietin-1 1 receptor OS=Mus musculus GN=Tek PE=1 SV=2 - [TIE2_MOUSE] & $0,000 E 0$ & $0,000 E 0$ & $0,000 E_{0}$ & 7,184E6 \\
\hline Q3TXS7 & $26 \mathrm{~S}$ proteasome non-ATPase regulatory subunit $1 \mathrm{OS}=$ Mus musculus $\mathrm{GN}=\mathrm{Psmd1} \mathrm{PE}=1 \mathrm{SV}=1$ - [PSMD1_MOUSE] & $0,000 \mathrm{E} 0$ & $0,000 E 0$ & $0,000 E 0$ & $8,673 E 5$ \\
\hline P07742 & Ribonucleoside-diphosphate reductase large subunit OS=Mus musculus GN=Rrm1 PE=1 SV=2 - [RIR1_MOUSE] & $0,000 \mathrm{E} 0$ & $0,000 \mathrm{E} 0$ & $0,000 \mathrm{E} 0$ & 3,894E6 \\
\hline P49962 & Signal recognition particle 9 kDa protein OS=Mus musculus GN=Srp9 PE=1 SV=2 - [SRP09_MOUSE] & $0,000 \mathrm{E} 0$ & $0,000 \mathrm{E} 0$ & $0,000 E 0$ & $5,072 E 6$ \\
\hline Q9QWY8-2 & Isoform 2 of Arf-GAP with SH3 domain, ANK repeat and PH domain-containing protein 1 OS=Mus musculus GN=Asap1 - [ASAP1_MOUSE] & $0,000 E_{0}$ & $0,000 E 0$ & $0,000 \mathrm{E} 0$ & $8,234 E 5$ \\
\hline Q3UH93 & Plexin-D1 OS=Mus musculus GN=Plxnd1 PE=1 SV=1 - [PLXD1_MOUSE] & $0,000 E_{0}$ & $0,000 E 0$ & $0,000 E_{0}$ & $6,711 E 6$ \\
\hline P32507 & Nectin-2 OS=Mus musculus GN=Nectin2 PE=1 SV=2 - [NECT2_MOUSE] & $0,000 E 0$ & $0,000 E 0$ & $0,000 E_{0}$ & $1,861 \mathrm{EE}$ \\
\hline Q8BKG3 & Inactive tyrosine-protein kinase 7 OS=Mus musculus GN=Ptk7 PE=1 SV=1 - [PTK7_MOUSE] & $0,000 \mathrm{E} 0$ & $0,000 E 0$ & $0,000 E_{0}$ & $7,024 E 6$ \\
\hline Q8C7Q4 & RNA-binding protein 4 OS=Mus musculus GN=Rbm4 PE=1 SV=1 - [RBM4_MOUSE] & $0,000 E 0$ & $0,000 E 0$ & $0,000 E 0$ & $7,181 E 6$ \\
\hline P35283 & Ras-related protein Rab-12 OS=Mus musculus GN=Rab12 PE=1 SV=3 - [RAB12_MOUSE] & $0,000 \mathrm{E} 0$ & $0,000 \mathrm{E} 0$ & $3,456 \mathrm{E}$ & $1,027 \mathrm{E}$ \\
\hline Q6PB66 & Leucine-rich PPR motif-containing protein, mitochondrial OS=Mus musculus GN=Lrpprc PE=1 SV=2 - [LPPRC_MOUSE] & $0,000 \mathrm{E} 0$ & $0,000 E_{0}$ & $0,000 E_{0}$ & $1,442 E 6$ \\
\hline Q8R3P2 & Probable E3 ubiquitin-protein ligase DTX2 OS=Mus musculus GN=Dt×2 PE=1 SV=2 - [DTX2_MOUSE] & $0,000 E_{0}$ & $0,000 \mathrm{E} 0$ & $0,000 \mathrm{E}_{0}$ & $2,626 \mathrm{E}$ \\
\hline A2APV2-2 & Isoform 2 of Formin-like protein 2 OS=Mus musculus GN=Fmnl2 - [FMNL__MOUSE] & $0,000 \mathrm{E} 0$ & $0,000 E 0$ & $0,000 E_{0}$ & $5,462 E 6$ \\
\hline Q9JJA4 & Ribosome biogenesis protein WDR12 OS=Mus musculus GN=Wdr12 PE=1 SV=1 - [WDR12_MOUSE] & $0,000 \mathrm{E} 0$ & $0,000 E 0$ & $0,000 E_{0}$ & 6,46555 \\
\hline Q6NSQ7 & Protein LTV1 homolog OS=Mus musculus GN=Ltv1 PE=1 SV=2 - [LTV1_MOUSE] & $0,000 \mathrm{E} 0$ & $0,000 \mathrm{E} 0$ & $0,000 \mathrm{E} 0$ & $2,306 \mathrm{E} 6$ \\
\hline Q8CB77 & Elongin-A OS=Mus musculus GN=Eloa PE=1 SV=3 - [ELOA1_MOUSE] & $0,000 E_{0}$ & $0,000 \mathrm{E} 0$ & $0,000 E_{0}$ & $2,946 E 6$ \\
\hline Q99L18 & Hepatocyte growth factor-regulated tyrosine kinase substrate $\mathrm{OS}=$ Mus musculus $\mathrm{GN}=\mathrm{HgS} \mathrm{PE}=1 \mathrm{SV}=2$ - [HGS_MOUSE] & $0,000 \mathrm{E} 0$ & $0,000 \mathrm{E} 0$ & $0,000 E_{0}$ & 9,902E6 \\
\hline Q9D832 & Dna] homolog subfamily B member 4 OS=Mus musculus GN=Dnajb4 PE=1 $\mathrm{SV}=1$ - [DNJB4_MOUSE] & $0,000 E 0$ & $0,000 E 0$ & $0,000 \mathrm{E}^{-}$ & $2,647 \pm 6$ \\
\hline Q8BRT1 & CLPP-associating protein 2 OS=Mus musculus GN=Clasp2 PE=1 SV=1 - [CLAP2_MOUSE] & $0,000 E 0$ & $0,000 E 0$ & $0,000 E 0$ & $1,931 \mathrm{EE}$ \\
\hline Q8ROAO & General transcription factor IIF subunit $2 \mathrm{OS}=$ Mus musculus GN=Gt2f2 PE=1 SV=1 - [T2FB_MOUSE] & $0,000 \mathrm{E} 0$ & $0,000 E 0$ & $0,000 \mathrm{E} 0$ & $1,547 \mathrm{E} 6$ \\
\hline Q9QZE5 & Coatomer subunit gamma-1 $\mathrm{OS}=$ Mus musculus GN=Copg1 PE=1 $1 \mathrm{SV}=1$ - [COPG1_MOUSE] & $0,000 E 0$ & $0,000 \mathrm{E} 0$ & $0,000 E_{0}$ & $3,576 \mathrm{E} 6$ \\
\hline 035593 & $26 \mathrm{~S}$ proteasome non-ATPase regulatory subunit $14 \mathrm{OS}=$ Mus musculus $\mathrm{GN}=\mathrm{Psmd} 14 \mathrm{PE}=1 \mathrm{SV}=2$ - [PSDE_MOUSE] & $0,000 E_{0}$ & $0,000 \mathrm{E} 0$ & $0,000 E_{0}$ & $3,273 E 6$ \\
\hline Q52KI8-2 & Isoform 2 of Serine/arginine repetitive matrix protein 1 OS=Mus musculus GN=Srrm1 - [SRRM1_MOUSE] & $0,000 \mathrm{E} 0$ & $0,000 E 0$ & $0,000 E_{0}$ & $3,972 E 6$ \\
\hline Q6PA11 & Breakpoint cluster region protein OS=Mus musculus GN=Bcr PE=1 SV=3 - [BCR_MOUSE] & $0,000 E 0$ & $0,000 E 0$ & $0,000 E_{0}$ & $1,494 \mathrm{E} 6$ \\
\hline P14211 & Calreticulin OS=Mus musculus GN=Calr PE=1 SV=1 - [CALR_MOUSE] & $0,000 E 0$ & $0,000 E 0$ & $0,000 E_{0}$ & 2,10956 \\
\hline Q9CPP0 & Nucleoplasmin-3 OS=Mus musculus GN=Npm3 PE=1 $1 \mathrm{SV}=3$ - [NPM3_MOUSE] & $0,000 \mathrm{E} 0$ & $0,000 \mathrm{E} 0$ & $0,000 E 0$ & $1,052 E 7$ \\
\hline Q8BHJ9 & Pre-mRNA-splicing factor SLU7 OS=Mus musculus GN=Slu7 PE=1 SV=1 - [SLU7_MOUSE] & $0,000 E_{0}$ & $0,000 E 0$ & $0,000 E_{0}$ & $3,418 E 6$ \\
\hline Q5ND28 & Scavenger receptor class $\mathrm{F}$ member $1 \mathrm{OS}=$ Mus musculus $\mathrm{GN}=\mathrm{Sc}$ carf1 $\mathrm{PE}=1 \mathrm{SV}=1$ - [SREC_MOUSE] & $0,000 E_{0}$ & $0,000 \mathrm{E} 0$ & $0,000 E_{0}$ & $1,314 E 6$ \\
\hline Q6NVF0-2 & Isoform 2 of Inositol polyphosphate 5-phosphatase OCRL-1 OS=Mus musculus GN=Ocrl- [OCRL_MOUSE] & $0,000 E 0$ & $0,000 E 0$ & $0,000 E 0$ & $1,591 E 6$ \\
\hline Q8CAF4-3 & Isoform 3 of NHS-like protein 1 OS=Mus musculus GN=NhsI1 - [NHSL1_MOUSE] & $0,000 E_{0}$ & $0,000 E_{0}$ & $0,000 E 0$ & $2,637 \mathrm{E} 6$ \\
\hline 054890 & Integrin beta-3 OS=Mus musculus GN=Itgb3 PE=1 SV=2 - [TTB3_MOUSE] & $0,000 \mathrm{E} 0$ & $0,000 \mathrm{E} 0$ & $0,000 \mathrm{E} 0$ & $7,201 E 6$ \\
\hline Q640N1 & Adipocyte enhancer-binding protein 1 OS=Mus musculus GN=Aebp1 PE=1SV=1 - [AEBP1_MOUSE] & $0,000 E 0$ & $0,000 \mathrm{E} 0$ & $0,000 E_{0}$ & $1,332 E 6$ \\
\hline Q9ES52-3 & Isoform 3 of Phosphatidylinositol 3,4,5-trisphosphate 5-phosphatase 1 OS=Mus musculus GN=Inpp5d - [SHIP1_MOUSE] & $0,000 E 0$ & $0,000 E 0$ & $0,000 E_{0}$ & $2,468 E 6$ \\
\hline Q9JKP5 & Muscleblind-like protein 1 OS=Mus musculus GN=Mbnl1 PE=1 SV=1 - [MBNL1_MOUSE] & $0,000 E 0$ & $0,000 E 0$ & $0,000 \mathrm{E} 0$ & $9,110 E 5$ \\
\hline Q80W68 & Kin of IRRE-like protein 1 OS=Mus musculus GN=Kirrel PE=1 SV=1 - [KIRR1_MOUSE] & $0,000 E 0$ & $0,000 E 0$ & $0,000 E_{0}$ & $3,203 E 6$ \\
\hline Q9D662 & Protein transport protein Sec23B OS=Mus musculus GN=Sec23b PE=1 SV=1 - [SC23B_MOUSE] & $0,000 E 0$ & $0,000 E 0$ & $0,000 E_{0}$ & 4,18556 \\
\hline P23949 & mRNA decay activator protein ZFP36L2 OS=Mus musculus GN=ZfF3612 PE=1 SV=2 - [TISD_MOUSE] & $0,000 \mathrm{E} 0$ & $0,000 \mathrm{E} 0$ & $0,000 E 0$ & $2,162 E 6$ \\
\hline Q9EPJ9 & ADP-ribosylation factor GTPase-activating protein 1 OS=Mus musculus GN=Arfgap1 PE=1 SV=2 - [ARFG1_MOUSE] & $0,000 E 0$ & $0,000 \mathrm{E} 0$ & $0,000 \mathrm{E} 0$ & $1,061 \mathrm{E} 6$ \\
\hline Q9C127 & Very-long-chain enoyl-CoA reductase OS=Mus musculus GN=Tecr PE=1 SV=1 - [TECR_MOUSE] & $0,000 \mathrm{E} 0$ & $0,000 \mathrm{E} 0$ & $0,000 \mathrm{E} 0$ & $1,247 \mathrm{E} 7$ \\
\hline Q8BMJ2 & Leucine--tRNA ligase, cytoplasmic OS=Mus musculus GN=Lars PE=1 SV=2 - [SYLC_MOUSE] & 0,000 E & 0,000E0 & $0,000 \mathrm{E} 0$ & 7,083E5 \\
\hline Q9R1Q6 & Transmembrane protein $176 \mathrm{~B}$ OS=Mus musculus GN=Tmem176b PE=1 SV=1 - [T176B_MOUSE] & $0,000 E 0$ & $0,000 E 0$ & $0,000 E_{0}$ & $3,373 E 6$ \\
\hline E9Q557 & Desmoplakin OS=Mus musculus GN=Dsp PE=1 SV=1 - [DESP_MOUSE] & $0,000 \mathrm{E} 0$ & $0,000 E 0$ & $8,274 E 6$ & $5,286 \mathrm{E} 6$ \\
\hline Q6ZPZ3-2 & Isoform 2 of Zinc finger CCCH domain-containing protein 4 OS=Mus musculus GN=Zc3h4 - [ZC3H4_MOUSE] & $0,000 \mathrm{E} 0$ & $0,000 \mathrm{E} 0$ & $0,000 \mathrm{E} 0$ & $2,382 E 6$ \\
\hline Q76LS9-2 & Isoform 2 of Ubiquitin carboxyl-terminal hydrolase MINDY-1 OS=Mus musculus GN=Fam63a - [FA63A_MOUSE] & $0,000 E_{0}$ & $0,000 \mathrm{E} 0$ & $0,000 E_{0}$ & 4,213E6 \\
\hline Q61214 & Dual specificity tyrosine-phosphorylation-regulated kinase 1A OS=Mus musculus GN=Dyrk1a PE=1 SV=1 - [DYR1A_MOUSE] & $0,000 \mathrm{EO}$ & 0,000E0 & $0,000 \mathrm{E} 0$ & $1,151 \mathrm{E} 6$ \\
\hline P35822 & Receptor-type tyrosine-protein phosphatase kappa OS=Mus musculus GN=Ptprk PE=1 SV=1 - [PTPRK_MOUSE] & $0,000 E 0$ & $0,000 E 0$ & $0,000 E_{0}$ & $3,513 \mathrm{E} 6$ \\
\hline Q99KY5 & Huntingtin-interacting protein 1-related protein $\mathrm{OS}=$ Mus musculus $\mathrm{GN}=\mathrm{Hip1r} \mathrm{PE}=1 \mathrm{SV}=2$ - [HIP1R_MOUSE] & $0,000 E 0$ & $0,000 E 0$ & $0,000 E_{0}$ & $1,761 \mathrm{E}$ \\
\hline Q6PFD9 & Nuclear pore complex protein Nup98-Nup96 OS=Mus musculus GN=Nup98 PE=1 SV=2 - [NUP98_MOUSE] & $0,000 \mathrm{E} 0$ & $0,000 E 0$ & $0,000 \mathrm{E} 0$ & $1,011 E 6$ \\
\hline Q5FWK3 & Rho GTPase-activating protein 1 OS=Mus musculus GN=Arhgap1 PE=1 SV=1 - [RHG01_MOUSE] & $0,000 E 0$ & $0,000 \mathrm{E} 0$ & $0,000 E 0$ & $1,877 \mathrm{E} 6$ \\
\hline Q8K3W3 & Protein CASC3 OS=Mus musculus GN=Casc3 PE=1 SV=3 - [CASC3_MOUSE] & $0,000 E 0$ & $0,000 \mathrm{E} 0$ & $0,000 E_{0}$ & $6,702 E 6$ \\
\hline Q60805 & Tyrosine-protein kinase Mer OS=Mus musculus GN=Mertk PE=1 SV=1 - [MERTK_MOUSE] & $0,000 E_{0}$ & $0,000 E 0$ & $0,000 E_{0}$ & $9,170 \mathrm{E} 6$ \\
\hline Q921Y2 & U3 small nucleolar ribonucleoprotein protein IMP3 OS=Mus musculus GN=Imp3 PE=2 SV=1 - [IMP3_MOUSE] & $0,000 \mathrm{E} 0$ & $0,000 E 0$ & $0,000 E 0$ & $4,491 \mathrm{E} 6$ \\
\hline P02468 & Laminin subunit gamma-1 $\mathrm{OS}=$ Mus musculus GN=Lamc1 PE=1 SV=2 - [LAMC1_MOUSE] & $0,000 E 0$ & $0,000 E 0$ & $0,000 E_{0}$ & $1,063 \mathrm{E} 6$ \\
\hline Q8CJ19 & [F-actin]-methionine sulfoxide oxidase MICAL3 OS=Mus musculus GN=Mical3 PE=1 SV=2 - [MICA3_MOUSE] & $0,000 \mathrm{E} 0$ & $0,000 E 0$ & $0,000 \mathrm{E} 0$ & $2,930 \mathrm{E} 6$ \\
\hline Q8BHD8 & Protein-L-isoaspartate O-methyltransferase domain-containing protein 2 OS=Mus musculus GN=Pcmtd2 PE=2 SV=1- [PCMD2_MOUSE] & $0,000 E_{0}$ & $0,000 \mathrm{E} 0$ & $0,000 E_{0}$ & $7,963 E 5$ \\
\hline Q9CQN1 & Heat shock protein $75 \mathrm{kDa}$, mitochondrial OS=Mus musculus GN=Trap1 PE=1 SV=1 - [TRAP1_MOUSE] & $0,000 E 0$ & $0,000 \mathrm{E} 0$ & $0,000 \mathrm{E} 0$ & 9,790E7 \\
\hline Q80UZ0 & FYVE, RhoGEF and PH domain-containing protein 5 OS=Mus musculus GN=Fgd5 PE=1 SV=2 - [FGD5_MOUSE] & $0,000 E 0$ & $0,000 E 0$ & $0,000 E_{0}$ & 4,53956 \\
\hline Q920B9 & FACT complex subunit SPT16 OS=Mus musculus GN=Supt16h PE=1 SV=2 - [SP16H_MOUSE] & $0,000 \mathrm{E} 0$ & $0,000 E 0$ & $0,000 E 0$ & $2,583 E 6$ \\
\hline Q91181 & RNA-binding protein 42 OS=Mus musculus GN=Rbm42 PE=1 $1 \mathrm{SV}=1$ - [RBM42_MOUSE] & $0,000 E 0$ & $0,000 E 0$ & $0,000 E_{0}$ & 1,86956 \\
\hline Q68FE6 & Protein FAM65A OS=Mus musculus GN=Fam65a PE=1 SV=2 - [FA65A_MOUSE] & $0,000 \mathrm{EO}$ & 0,000E0 & $0,000 E 0$ & $2,239 \mathrm{E} 6$ \\
\hline Q5SSZ5-2 & Isoform 2 of Tensin-3 OS=Mus musculus GN=Tns3 - [TENS3_MOUSE] & $0,000 E 0$ & $0,000 E 0$ & $0,000 E_{0}$ & $3,158 E 7$ \\
\hline P70227 & Inositol 1,4,5-trisphosphate receptor type 3 OS=Mus musculus GN=Itpr3 PE=1 SV=3 - [ITPR3_MOUSE] & $0,000 E 0$ & $0,000 E 0$ & $0,000 E_{0}$ & $4,207 E 6$ \\
\hline Q9DAX2 & Phospholipid phosphatase 2 OS=Mus musculus GN=PIpp2 PE=1 SV=1 - [PLPP2_MOUSE] & $0,000 E 0$ & $0,000 E 0$ & $0,000 E_{0}$ & 6,375E5 \\
\hline Q00560 & Interleukin-6 receptor subunit beta OS=Mus musculus GN=Il6st PE=1 SV=2 - [IL6RB_MOUSE] & $0,000 E_{0}$ & $0,000 E 0$ & $0,000 E_{0}$ & $1,181 E 6$ \\
\hline Q00612 & Glucose-6-phosphate 1-dehydrogenase X OS=Mus musculus GN=G6pdx PE=1 SV=3 - [G6PD1_MOUSE] & $0,000 \mathrm{E} 0$ & $0,000 \mathrm{E} 0$ & $0,000 \mathrm{E} 0$ & 1,193E6 \\
\hline P51432 & 1-phosphatidylinositol 4,5-bisphosphate phosphodiesterase beta-3 OS=Mus musculus GN=Plcb3 PE=1 SV=2 - [PLCB3_MOUSE] & $0,000 \mathrm{E} 0$ & $0,000 \mathrm{E} 0$ & $0,000 \mathrm{E} 0$ & $2,362 E 6$ \\
\hline 035680 & 285 ribosomal protein S12, mitochondrial OS=Mus musculus GN=Mrps12 PE=2 SV=1 - [RT12_MOUSE] & $0,000 \mathrm{E} 0$ & $0,000 \mathrm{E} 0$ & $0,000 E_{0}$ & $5,814 E 6$ \\
\hline Q8R5G7 & Arf-GAP with Rho-GAP domain, ANK repeat and PH domain-containing protein 3 OS=Mus musculus GN=Arap3 PE=1 SV=3 - [ARAP3_MOUSE] & $0,000 E_{0}$ & $0,000 E 0$ & $0,000 E_{0}$ & $1,388 E 6$ \\
\hline A2RT67 & DENN domain-containing protein 3 OS=Mus musculus GN=Dennd3 PE=1 SV=2 - [DEND3_MOUSE] & $0,000 \mathrm{E} 0$ & $0,000 \mathrm{E} 0$ & $0,000 \mathrm{E} 0$ & $1,304 E 6$ \\
\hline Q8R010 & Aminoacyl tRNA synthase complex-interacting multifunctional protein 2 OS=Mus musculus GN=Aimp2 PE=1 SV=2 - [AIMP2_MOUSE] & $0,000 \mathrm{E} 0$ & $0,000 E 0$ & $0,000 E_{0}$ & 4,011E6 \\
\hline Q8R4Y4-2 & Isoform 2 of Stabilin-1 OS=Mus musculus GN=Stab1 - [STAB1_MOUSE] & $0,000 \mathrm{E} 0$ & $0,000 E 0$ & $0,000 \mathrm{E} 0$ & $2,266 \mathrm{E} 7$ \\
\hline BIAXH1 & NHS-like protein 2 OS=Mus musculus GN=NhsI2 PE=1 SV=1 - [NHSL2_MOUSE] & $0,000 E_{0}$ & $0,000 \mathrm{E} 0$ & $0,000 E_{0}$ & $5,183 E 6$ \\
\hline AZA4P0 & ATP-dependent RNA helicase DHX8 OS=Mus musculus GN=Dhx8 PE=2 SV=1 - [DHX8_MOUSE] & $0,000 E_{0}$ & $0,000 E_{0}$ & $0,000 E_{0}$ & $4,776 \mathrm{E} 6$ \\
\hline Q02105 & Complement C1q subcomponent subunit C OS=Mus musculus GN=C1qc PE=1 SV=2 - [C1QC_MOUSE] & $0,000 E 0$ & $0,000 E 0$ & $0,000 E_{0}$ & $1,832 E 7$ \\
\hline 054734 & Dolichyl-diphosphooligosaccharide--protein glycosyltransferase 48 kDa subunit OS=Mus musculus GN=Ddost PE=1 SV=2 - [OST48_MOUSE] & $0,000 E 0$ & $0,000 E 0$ & $0,000 \mathrm{E} 0$ & $1,655 \mathrm{E} 6$ \\
\hline Q3TTA7-2 & Isoform 2 of E3 ubiquitin-protein ligase CBL-B OS=Mus musculus GN=Cblb - [CBLB_MOUSE] & $0,000 \mathrm{E} 0$ & $0,000 \mathrm{E} 0$ & $0,000 \mathrm{E}_{0}$ & $1,702 E 6$ \\
\hline Q80225 & Oral-facial-digital syndrome 1 protein homolog OS=Mus musculus GN=Ofd1 PE=1 SV=1 - [OFD1_MOUSE] & $0,000 \mathrm{E} 0$ & $0,000 \mathrm{E} 0$ & $0,000 \mathrm{E} 0$ & 6,32955 \\
\hline Q9נIII-2 & Isoform 2 of DAZ-associated protein 1 OS=Mus musculus GN=Dazap1 - [DAZP1_MOUSE] & $0,000 E 0$ & $0,000 E 0$ & $0,000 \mathrm{E} 0$ & $5,965 \mathrm{E} 6$ \\
\hline Q6DVA0 & LEM domain-containing protein $2 \mathrm{OS}=$ Mus muscullus GN=Lemd2 PE=1 SV=1 - [LEMD2_MOUSE] & $0,000 E 0$ & $0,000 E 0$ & $0,000 E 0$ & $8,142 E 5$ \\
\hline B2RU80 & Receptor-type tyrosine-protein phosphatase beta OS=Mus musculus GN=Ptprb PE=1 SV=1 - [PTPRB_MOUSE] & $0,000 E 0$ & $0,000 E 0$ & $0,000 E_{0}$ & $2,030 \mathrm{E} 6$ \\
\hline Q9CZD5 & Translation initiation factor IF-3, mitochondrial OS=Mus musculus GN=Mtif3 PE=1 SV=1 - [IF3M_MOUSE] & $0,000 E 0$ & $0,000 E 0$ & $0,000 E_{0}$ & $2,908 E 6$ \\
\hline
\end{tabular}




\begin{tabular}{|c|c|c|c|c|c|}
\hline Q01705-4 & Isoform 4 of Neurogenic locus notch homolog protein 1 OS=Mus musculus GN=Notch1 - [NOTC1_MOUSE] & $0,000 \mathrm{EO}^{-}$ & $0,000 \mathrm{E} 0$ & $0,000 \mathrm{E} 0$ & $1,475 E 7$ \\
\hline Q8JZW4 & Copine-5 OS=Mus musculus GN=CPne5 PE=1 SV=1- [CPNE5_MOUSE] & $0,000 \mathrm{E} 0$ & $0,000 \mathrm{E} 0$ & $0,000 \mathrm{E} 0$ & $2,218 \mathrm{EE}$ \\
\hline Q9QXZ0 & Microtubule-actin cross-linking factor $1 \mathrm{OS}=$ Mus musculus $\mathrm{GN}=$ Macf1 PE=1 $\mathrm{SV}=2$ - [MACF1_MOUSE] & $0,000 E 0$ & $0,000 E 0$ & $0,000 \mathrm{E}_{0}$ & $4,223 \mathrm{E} 6$ \\
\hline Q80U78-2 & Isoform 2 of Pumilio homolog 1 OS=Mus musculus GN=Pum1 - [PUM1_MOUSE] & $0,000 \mathrm{E} 0$ & $0,000 E 0$ & $0,000 \mathrm{E}^{-}$ & 1,253E6 \\
\hline Q07139-2 & Isoform 2 of Protein ECT2 OS=Mus musculus GN=Ect2 - [ECT2_MOUSE] & $0,000 E 0$ & $0,000 E 0$ & $0,000 \mathrm{E} 0$ & $5,244 E 5$ \\
\hline P61079 & Ubiquitin-conjugating enzyme E2 D3 OS=Mus musculus GN=Ube2d3 PE=1 SV=1- [UB2D3_MOUSE] & $0,000 \mathrm{E} 0$ & $0,000 \mathrm{E} 0$ & $0,000 \mathrm{E} 0$ & $1,082 \mathrm{E} 7$ \\
\hline Q8C5L3-3 & Isoform 3 of CCR4-NOT transcription complex subunit 2 OS=Mus musculus GN=Cnot2 - [CNOT2_MOUSE] & $0,000 E_{0}$ & $0,000 E 0$ & $0,000 E_{0}$ & 1,973E6 \\
\hline Q91WM1-2 & Isoform 2 of Spermatid perinuclear RNA-binding protein OS=Mus musculus GN=Strbp - [STRBP_MOUSE] & 0,000E0 & 0,000E0 & $0,0000_{0}$ & 1,346E7 \\
\hline 035660 & Glutathione S-transferase Mu 6 OS=Mus musculus GN=Gstm6 PE=1 SV=4 - [GSTM6_MOUSE] & $0,000 \mathrm{E} 0$ & $0,000 E 0$ & $0,000 \mathrm{E}$ & 5,973E5 \\
\hline Q99MS7 & EH domain-binding protein 1-like protein 1 OS=Mus musculus GN=Ehbp111 PE=1 SV=1 - [EH1L1_MOUSE] & $0,000 \mathrm{E} 0$ & $0,000 \mathrm{E} 0$ & $0,000 \mathrm{E} 0$ & 3,964E6 \\
\hline P23463 & Homeobox protein Hox-D8 OS=Mus musculus GN=Hoxd8 PE=3 SV=4 - [HXD8_MOUSE] & $0,000 \mathrm{E} 0$ & $0,000 E 0$ & $0,000 \mathrm{E}_{0}$ & $2,880 \mathrm{E} 6$ \\
\hline P83093 & Stromal interaction molecule 2 OS=Mus musculus GN=Stim2 PE=1 SV=2 - [STIM2_MOUSE] & $0,000 E_{0}$ & $0,000 \mathrm{E} 0$ & $0,000 \mathrm{E}_{0}$ & 1,603E6 \\
\hline Q61001 & Laminin subunit alpha-5 OS=Mus musculus GN=Lama5 PE=1 SV=4 - [LAMA5_MOUSE] & $0,000 \mathrm{E} 0$ & $0,000 E 0$ & $0,000 \mathrm{E} 0$ & 1,300E6 \\
\hline P35821 & Tyrosine-protein phosphatase non-receptor type $10 \mathrm{OS}=$ Mus musculus GN=Ptpn1 PE=1 SV=2 - [PTN1_MOUSE] & $0,000 \mathrm{E}_{0}$ & $0,000 \mathrm{E} 0$ & $0,000 \mathrm{E}_{0}$ & $8,743 E 5$ \\
\hline Q80SW1 & Putative adenosylhomocysteinase $2 \mathrm{OS}=$ Mus musculus GN=Ahcyl1 PE=1 SV=1 - [SAHH2_MOUSE] & $0,000 \mathrm{E} 0$ & $0,000 \mathrm{E} 0$ & $0,000 \mathrm{E} 0$ & $2,795 \mathrm{E} 6$ \\
\hline Q9DC69 & NADH dehydrogenase [ubiquinone] 1 alpha subcomplex subunit 9, mitochondrial OS=Mus musculus GN=Ndufa9 PE=1 $1 \mathrm{SV}=2$ - [NDUA9_MOUSE] & $0,000 \mathrm{E} 0$ & 0,000E0 & $0,0000_{0}$ & $1,643 \mathrm{E} 6$ \\
\hline Q99KN1-2 & Isoform 2 of Arrestin domain-containing protein 1 OS=Mus musculus GN=Arrdc1 - [ARRD1_MOUSE] & $0,000 \mathrm{E} 0$ & $0,000 \mathrm{E} 0$ & $0,000 \mathrm{E} 0$ & 9,543E5 \\
\hline Q80Y56 & Rabenosyn-5 OS=Mus musculus GN=Rbsn PE=1 SV=1 - [RBNS5_MOUSE] & $0,000 E 0$ & $0,000 E 0$ & $0,000 \mathrm{E}_{0}$ & $9,590 \mathrm{E} 5$ \\
\hline Q64430 & Copper-transporting ATPase 1 OS=Mus musculus GN=Atp7a PE=1 SV=3 - [ATP7A_MOUSE] & $0,000 E 0$ & $0,000 E 0$ & $0,000 E 0$ & 1,294E6 \\
\hline Q8BG79 & CWF19-like protein 2 OS=Mus musculus GN=Cwf1912 PE=1 SV=1 - [C19L2_MOUSE] & $0,000 E 0$ & $0,000 E 0$ & $0,000 E_{0}$ & $7,675 E 5$ \\
\hline P35918 & Vascular endothelial growth factor receptor $2 \mathrm{OS}=$ Mus musculus GN=Kdr PE=1 SV=1 - [VGFR2_MOUSE] & $0,000 \mathrm{E} 0$ & $0,000 \mathrm{E} 0$ & $0,000 \mathrm{E} 0$ & $1,139 \mathrm{E} 7$ \\
\hline Q8BZX4 & Splicing regulatory glutamine//ysine-rich protein 1 OS=Mus musculus GN=Srek1 PE=2 SV=1 - [SREK1_MOUSE] & $0,000 E_{0}$ & $0,000 \mathrm{E} 0$ & $0,000 E_{0}$ & $1,378 E 6$ \\
\hline Q63850 & Nuclear pore glycoprotein p62 OS=Mus musculus GN=Nup62 PE=1 SV=2 - [NUP62_MOUSE] & $0,000 \mathrm{E} 0$ & $0,000 E 0$ & $0,000 E_{0}$ & $1,735 \mathrm{E} 6$ \\
\hline Q88KI2 & Trinucleotide repeat-containing gene $6 \mathrm{~B}$ protein $\mathrm{OS}=$ Mus musculus $\mathrm{GN}=\mathrm{TnR} 6 \mathrm{bb} \mathrm{PE}=1 \mathrm{SV}=2$ - [TNR6B_MOUSE] & $0,000 E_{0}$ & $0,000 \mathrm{E} 0$ & $0,000 \mathrm{E}_{0}$ & $2,376 \mathrm{E} 6$ \\
\hline P46664 & Adenylosuccinate synthetase isozyme 2 OS=Mus musculus $\mathrm{GN}=\mathrm{AdsS} \mathrm{PE}=1 \mathrm{SV}=2$ - [PURA2_MOUSE] & $0,000 E 0$ & $0,000 E 0$ & $0,0000_{0}$ & $2,239 E 6$ \\
\hline Q8BM55 & Transmembrane protein 214 OS=Mus musculus GN=Tmem214 PE=1 SV=1 - [TM214_MOUSE] & $0,000 \mathrm{E} 0$ & $0,000 \mathrm{E} 0$ & $0,000 \mathrm{E}_{0}$ & $2,949 E 6$ \\
\hline Q9D2R0 & Acetoacetyl-CoA synthetase OS=Mus musculus GN=Aacs PE $=1$ SV=1 - [AACS_MOUSE] & $0,000 \mathrm{E} 0$ & $0,000 \mathrm{E} 0$ & $0,000 \mathrm{E} 0$ & 1,939E6 \\
\hline Q921T1 & AP-3 complex subunit beta-1 $\mathrm{OS}=$ Mus musculus GN=AP3b1 PE=1 SV=2 - [AP3B1_MOUSE] & $0,000 E_{0}$ & $0,000 \mathrm{E} 0$ & $0,000 \mathrm{E}^{0}$ & $3,649 \mathrm{E} 6$ \\
\hline AZAAY5 & SH3 and PX domain-containing protein 2B OS=Mus musculus GN=Sh3pxd2b PE=1 SV=1 - [SPD2B_MOUSE] & $0,000 \mathrm{E} 0$ & $0,000 \mathrm{E} 0$ & $0,000 \mathrm{E}_{0}$ & $1,672 E 6$ \\
\hline Q8K2B3 & Succinate dehydrogenase [ubiquinone] flavoprotein subunit, mitochondrial OS=Mus musculus GN=Sdha PE=1 SV=1 - [SDHA_MOUSE] & $0,000 E 0$ & $0,000 E 0$ & $0,000 \mathrm{E}_{0}$ & $3,018 \mathrm{E} 6$ \\
\hline Q3UFM5 & Nucleolar MIF4G domain-containing protein 1 OS=Mus musculus GN=Nom1 PE=1 SV=2 - [NOM1_MOUSE] & 0,000E0 & 0,000E0 & $0,0000_{0}$ & 4,975E5 \\
\hline Q9D706 & RNA polymerase II-associated protein 3 OS=Mus musculus GN=Rpap3 PE=1 SV=1 - [RPAP3_MOUSE] & $0,000 E 0$ & $0,000 E 0$ & $0,000 \mathrm{E} 0$ & $3,438 E 6$ \\
\hline Q511X5 & RelA-associated inhibitor OS=Mus musculus GN=Ppp1r131 PE=1 SV=1 - [IASPP_MOUSE] & $0,000 E 0$ & $0,000 \mathrm{E} 0$ & $0,000 E_{0}$ & $8,316 E 5$ \\
\hline Q6ZPE2-2 & Isoform 2 of Myotubularin-related protein 5 OS=Mus musculus GN=Sbf1 - [MTMR5_MOUSE] & $0,000 E_{0}$ & $0,000 \mathrm{E} 0$ & $0,000 E_{0}$ & $1,716 \mathrm{E} 6$ \\
\hline Q3UPH7-3 & Isoform 3 of Rho guanine nucleotide exchange factor 40 OS=Mus musculus GN=Arhgef40 - [ARH40_MOUSE] & $0,000 E 0$ & $0,000 E 0$ & 0,000E0 & 2,345E6 \\
\hline Q88X57-3 & Isoform 3 of PX domain-containing protein kinase-like protein OS=Mus musculus GN=Pxk - [PXK_MOUSE] & $0,000 \mathrm{E} 0$ & $0,000 E 0$ & $0,000 \mathrm{E}_{0}$ & $1,065 E 6$ \\
\hline Q8CIP4-2 & Isoform 2 of MAP/microtubule affinity-regulating kinase 4 OS=Mus musculus GN=Mark4 - [MARK4_MOUSE] & $0,000 \mathrm{E} 0$ & 0,000E0 & $0,0000_{0}$ & $9,286 \mathrm{E} 6$ \\
\hline Q80093 & Nuclear pore complex protein Nup214 OS=Mus musculus GN=Nup214 PE=1 SV=2 - [NU214_MOUSE] & $0,000 E 0$ & $0,000 E 0$ & $0,000 E 0$ & $5,193 E 5$ \\
\hline Q6ZQ38 & Cullin-associated NEDD8-dissociated protein 1 OS=Mus musculus GN=Cand1 PE=1 SV=2 - [CAND1_MOUSE] & $0,000 E 0$ & $0,000 E 0$ & $0,0000_{0}$ & 1,241E6 \\
\hline Q80SU7 & Interferon-induced very large GTPase 1 OS=Mus musculus GN=Gvin1 PE=1 SV=1- [GVIN1_MOUSE] & $0,000 \mathrm{E} 0$ & $0,000 \mathrm{E} 0$ & $0,000 \mathrm{E}^{\circ}$ & $1,487 \mathrm{E} 6$ \\
\hline Q99LI5 & Zinc finger protein 281 OS=Mus musculus GN=Znf281 PE=1 1 SV=1 - [ZN281_MOUSE] & $0,000 \mathrm{E} 0$ & $0,000 \mathrm{E}$ & $0,000 E 0$ & $2,058 \mathrm{E} 6$ \\
\hline Q9R0U0-3 & Isoform 3 of Serine/arginine-rich splicing factor 10 OS=Mus musculus GN=Srsf10 - [SRS10_MOUSE] & $0,000 E 0$ & $0,000 E_{0}$ & 0,000E0 & 1,277E6 \\
\hline P47226-2 & Isoform TES1 of Testin OS=Mus musculus GN=Tes $-[$ TES_MOUSE] & $0,000 \mathrm{E} 0$ & $0,000 E 0$ & $0,000 \mathrm{E} 0$ & $6,851 E 5$ \\
\hline Q6ZQ73 & Cullin-associated NEDD8-dissociated protein 2 OS=Mus musculus GN=Cand2 PE=1 $\mathrm{SV}=2$ - [CAND2_MOUSE] & $0,000 \mathrm{E} 0$ & $0,000 \mathrm{E} 0$ & $0,000 \mathrm{E}_{0}$ & $2,120 \mathrm{E} 6$ \\
\hline Q8BP22 & Protein FAM92A1 OS=Mus musculus GN=Fam92a1 PE=1 SV=1 - [F92A1_MOUSE] & $0,000 \mathrm{E} 0$ & $0,000 \mathrm{E} 0$ & $0,000 E_{0}$ & 1,141E6 \\
\hline P14873 & Microtubule-associated protein $1 \mathrm{~B}$ OS=Mus musculus GN=Map1b PE=1 SV=2 - [MAP1B_MOUSE] & $0,000 \mathrm{E} 0$ & $0,000 \mathrm{E} 0$ & $2,559 \mathrm{E} 6$ & $2,404 E 6$ \\
\hline Q8C0D4 & Rho GTPase-activating protein 12 OS=Mus musculus GN=Arhgap12 PE=1 SV=2 - [RHG12_MOUSE] & $0,000 \mathrm{EO}$ & $0,000 \mathrm{EO}$ & 0,000E0 & $\begin{array}{ll}1,616 \mathrm{E} 6 \\
\end{array}$ \\
\hline Q8BU30 & Isoleucine--tRNA ligase, cytoplasmic OS=Mus musculus GN=Iars PE=1 SV=2 - [SYIC_MOUSE] & $0,000 E 0$ & $0,000 \mathrm{E} 0$ & $0,000 \mathrm{E} 0$ & $5,789 \mathrm{E}$ \\
\hline P70398 & Probable ubiquitin carboxyl-terminal hydrolase FAF-X OS=Mus musculus $\mathrm{GN}=U \mathrm{Usp9x}$ PE=1 SV=2 - [USP9X_MOUSE] & $0,000 \mathrm{E} 0$ & $0,000 \mathrm{E} 0$ & $8,255 E 5$ & $2,010 \mathrm{E} 6$ \\
\hline Q8K389 & CDK5 regulatory subunit-associated protein $20 \mathrm{OS}=$ Mus musculus GN=Cdk5rap2 PE=1 SV=3 - [CK5P2_MOUSE] & $0,000 \mathrm{E} 0$ & $0,000 \mathrm{E} 0$ & $0,000 \mathrm{E} 0$ & $8,224 E 5$ \\
\hline Q8K2033 & Enhancer of mRNA-decapping protein 3 OS=Mus musculus GN=Edc3 PE=1 SV=1 - [EDC3_MOUSE] & $0,000 \mathrm{E} 0$ & $0,000 \mathrm{E} 0$ & $0,000 \mathrm{E} 0$ & $1,428 E 6$ \\
\hline P222777 & Plasminogen activator inhibitor $10 \mathrm{OS}=$ Mus musculus GN=Serpine1 PE=1 SV=1 - [PAI1_MOUSE] & $0,000 \mathrm{E}$ & 0,000E0 & $0,0000_{0}$ & $4,451 \mathrm{E} 6$ \\
\hline FGZDS4 & Nucleoprotein TPR OS=Mus musculus GN=Tpr PE=1 SV=1 - [TPR_MOUSE] & $0,000 \mathrm{E} 0$ & $0,000 E 0$ & $0,000 \mathrm{E}_{0}$ & $7,245 E 5$ \\
\hline Q8C5N3-2 & Isoform 2 of Pre-mRNA-splicing factor CWC22 homolog OS=Mus musculus GN=CWC22 - [CWC22_MOUSE] & $0,000 \mathrm{E} 0$ & $0,000 E 0$ & $0,000 \mathrm{E} 0$ & 6,254E6 \\
\hline Q9DBHO & NEDD4-like E3 ubiquitin-protein ligase WWP2 OS=Mus musculus GN=WWP2 PE=1 SV=1 - [WWP2_MOUSE] & $0,000 \mathrm{E} 0$ & $0,000 \mathrm{E} 0$ & $0,000 \mathrm{E} 0$ & $2,994 E 6$ \\
\hline P10404 & MLV-related proviral Env polyprotein OS=Mus musculus PE=1 SV=3 - [ENV1_MOUSE] & $0,000 \mathrm{E} 0$ & $0,000 E 0$ & $0,000 \mathrm{E}_{0}$ & $4,281 E 6$ \\
\hline P24270 & Catalase $\mathrm{OS}=$ Mus musculus $\mathrm{GN}=\mathrm{Cat} P \mathrm{PE}=1 \mathrm{SV}=4$ - [CATA_MOUSE] & $0,000 \mathrm{E} 0$ & $0,000 \mathrm{E} 0$ & $0,000 \mathrm{E}^{\circ}$ & $2,870 \mathrm{E} 6$ \\
\hline Q9R1Q9 & V-type proton ATPase subunit S1 OS=Mus musculus GN=Atp6ap1 PE=1 SV=1 - [VAS1_MOUSE] & $0,000 E 0$ & $0,000 E 0$ & $0,000 \mathrm{E}_{0}$ & $1,220 \mathrm{E} 6$ \\
\hline Q64343 & ATP-binding cassette sub-family G member 1 OS=Mus musculus GN=Abcg1 PE=1 SV=1- [ABCG1_MOUSE] & $0,000 \mathrm{E}_{0}$ & $0,000 \mathrm{E} 0$ & $0,000 \mathrm{E}_{0}$ & $5,462 E 6$ \\
\hline Q06806 & Tyrosine-protein kinase receptor Tie-1 OS=Mus musculus GN=Tie1 PE=1 SV=3 - [TIE1_MOUSE] & $0,000 \mathrm{E} 0$ & $0,000 \mathrm{E} 0$ & $0,000 \mathrm{E} 0$ & $8,554 E 6$ \\
\hline Q8BKF1 & DNA-directed RNA polymerase, mitochondrial OS=Mus musculus GN=Polrmt PE $=1 \mathrm{SV}=1$ - [RPOM_MOUSE] & $0,000 E 0$ & $0,000 E 0$ & $0,000 E 0$ & 2,080E6 \\
\hline 054825 & Bystin OS=Mus musculus GN=Bys PE=1 SV=3 - [BYST_MOUSE] & $0,000 \mathrm{E} 0$ & $0,000 \mathrm{E} 0$ & $0,000 E_{0}$ & $7,798 E 5$ \\
\hline Q3U096 & Serine/threonine-protein kinase MRCK alpha OS=Mus musculus GN=Cdc42bpa PE=1 SV=2 - [MRCKA_MOUSE] & $0,000 E 0$ & $0,000 E 0$ & $0,000 E_{0}$ & $2,825 E 6$ \\
\hline Q6DFV3 & Rho GTPase-activating protein 21 OS=Mus musculus GN=Arhgap21 PE=1 SV=1 - [RHG21_MOUSE] & $0,000 E 0$ & $0,000 E 0$ & $0,000 E 0$ & $2,212 E 6$ \\
\hline Q8C156 & Condensin complex subunit $2 \mathrm{OS}=$ Mus musculus $\mathrm{GN}=\mathrm{Ncaph} \mathrm{PE}=1 \mathrm{SV}=1$ - [CND2_MOUSE] & $0,000 \mathrm{E} 0$ & $0,000 \mathrm{E} 0$ & $0,000 \mathrm{E} 0$ & $2,078 E 6$ \\
\hline B2RX88-2 & Isoform 2 of Centrosome and spindle pole associated protein 1 OS=Mus musculus GN=CSpp1 - [CSPP1_MOUSE] & $0,000 \mathrm{E} 0$ & $0,000 \mathrm{E} 0$ & $0,000 \mathrm{E} 0$ & 1,909E6 \\
\hline Q8BHY3-2 & Isoform 2 of Anoctamin-1 $\mathrm{OS}=$ Mus musculus GN=Ano1 - [ANO1_MOUSE] & $0,000 E_{0}$ & $0,000 E_{0}$ & $0,000 E_{0}$ & $1,282 E 6$ \\
\hline Q62077 & 1-phosphatidylinositol 4,5-bisphosphate phosphodiesterase gamma-1 OS=Mus musculus GN=Plcg1 PE=1 SV=2 - [PLCG1_MOUSE] & $0,000 E_{0}$ & $0,000 E_{0}$ & $0,000 E_{0}$ & 1,321E6 \\
\hline Q8BPM0-2 & Isoform 2 of Disheveled-associated activator of morphogenesis 1 OS=Mus musculus GN=Daam1 - [DAAM1_MOUSE] & $0,000 \mathrm{E} 0$ & $0,000 \mathrm{E} 0$ & $0,000 \mathrm{E}_{0}$ & $1,516 \mathrm{E} 6$ \\
\hline Q3U0V2 & Tumor necrosis factor receptor type 1-associated DEATH domain protein OS=Mus musculus GN=Tradd PE=1 SV=1 - [TRADD_MOUSE] & $0,000 \mathrm{E} 0$ & $0,000 \mathrm{E} 0$ & $0,000 \mathrm{E} 0$ & $2,135 E 5$ \\
\hline Q80TP3 & E3 ubiquitin-protein ligase UBR5 OS=Mus musculus GN=Ubr5 PE=1 SV=2 - [UBR5_MOUSE] & $0,000 \mathrm{E} 0$ & $0,000 \mathrm{E} 0$ & $0,000 \mathrm{E} 0$ & 1,013E6 \\
\hline Q61233 & Plastin-2 OS=Mus musculus GN=Lcp1 PE=1 SV=4 - [PLSL_MOUSE] & $0,000 \mathrm{E} 0$ & $0,000 \mathrm{E} 0$ & $0,000 \mathrm{E} 0$ & $6,535 \mathrm{E} 6$ \\
\hline Q8VE97 & Serine/arginine-rich splicing factor 4 OS=Mus musculus GN=Srsf4 PE=2 SV=1 - [SRSF4_MOUSE] & $0,000 E_{0}$ & $0,000 \mathrm{E} 0$ & $0,000 \mathrm{E}^{0}$ & $1,028 E 8$ \\
\hline 008911 & Mitogen-activated protein kinase 12 OS=Mus musculus GN=Mapk12 PE=1 SV=1 - [MK12_MOUSE] & $0,000 \mathrm{E} 0$ & $0,000 \mathrm{E} 0$ & $0,000 \mathrm{E}_{0}$ & $4,305 E 6$ \\
\hline Q4U2R1 & E3 ubiquitin-protein ligase HERC2 OS=Mus musculus GN=Herc2 PE=1 SV=3 - [HERC2_MOUSE] & $0,000 E 0$ & $0,000 E 0$ & $0,000 E_{0}$ & 1,073E6 \\
\hline P59764 & Dedicator of cytokinesis protein 4 OS=Mus musculus GN=Dock4 PE=1 SV=1 - [DOCK4_MOUSE] & $0,000 \mathrm{E} 0$ & $0,000 E 0$ & $0,000 \mathrm{E}_{0}$ & $1,700 E 6$ \\
\hline P47811-4 & Isoform 4 of Mitogen-activated protein kinase 14 OS=Mus musculus GN=Mapk14 - [MK14_MOUSE] & $0,000 \mathrm{E} 0$ & $0,000 \mathrm{E} 0$ & $0,000 \mathrm{E} 0$ & 4,305E6 \\
\hline Q9JMD0-4 & Isoform 4 of BUB3-interacting and GLEBS motif-containing protein ZNF207 OS=Mus musculus GN=Znf207 - [ZN207_MOUSE] & $0,000 E 0$ & $0,000 \mathrm{E} 0$ & $0,000 E_{0}$ & 9,455E6 \\
\hline Q8CCP0 & Nuclear export mediator factor Nemf OS=Mus musculus $\mathrm{GN}=$ Nemf PE=1 SV=2 - [NEMF_MOUSE] & $0,000 E_{0}$ & $0,000 E 0$ & $0,000 E_{0}$ & $3,048 E 6$ \\
\hline Q8BT60 & Copine-3 OS=Mus musculus GN=CPne3 PE=1 SV=2 - [CPNE3_MOUSE] & $0,000 \mathrm{E} 0$ & $0,000 \mathrm{E} 0$ & $0,000 \mathrm{E} 0$ & $2,218 E 6$ \\
\hline Q6P5F9 & Exportin-1 OS=Mus musculus GN=Xpo1 PE=1 SV=1- [XPO1_MOUSE] & $0,000 \mathrm{E} 0$ & $0,000 \mathrm{E} 0$ & $0,000 \mathrm{E} 0$ & $2,028 E 6$ \\
\hline P27460 & Gag polyprotein OS=Cas-Br-E murine leukemia virus GN=gag PE=3 SV=3 - [GAG_MLVCB] & $0,000 \mathrm{E} 0$ & $0,000 \mathrm{E} 0$ & $0,000 \mathrm{E} 0$ & 3,382E6 \\
\hline E9Q5K9-3 & Isoform 3 of YTH domain-containing protein 1 OS=Mus musculus GN=Ythdc1 - [YTDC1_MOUSE] & $0,000 \mathrm{E} 0$ & $0,000 \mathrm{E} 0$ & $0,0000_{0}$ & $2,534 E 6$ \\
\hline Q8K230 & 1-phosphatidylinositol 4,5-bisphosphate phosphodiesterase delta-3 OS=Mus musculus GN=PIcd3 PE=1 SV=2 - [PLCD3_MOUSE] & $0,000 \mathrm{E} 0$ & $0,000 \mathrm{E} 0$ & $0,000 \mathrm{E} 0$ & $9,401 E 5$ \\
\hline Q5SUA5 & Unconventional myosin-Ig OS=Mus musculus GN=Myo1g PE=1 SV=1 - [MYO1G_MOUSE] & $0,000 \mathrm{E} 0$ & $0,000 \mathrm{E} 0$ & $0,000 \mathrm{E}_{0}$ & $1,010 \mathrm{E} 7$ \\
\hline 055103 & Periaxin OS=Mus musculus GN=Prx PE=1 SV=1 - [PRAX_MOUSE] & $0,000 \mathrm{E} 0$ & $0,000 \mathrm{E} 0$ & $0,000 \mathrm{E} 0$ & $3,868 \mathrm{E} 6$ \\
\hline 088532 & Zinc finger RNA-binding protein OS=Mus musculus GN=Zfr PE=1 SV=2 - [ZFR_MOUSE] & $0,000 \mathrm{E} 0$ & $0,000 E 0$ & $0,000 \mathrm{E}_{0}$ & 1,465E6 \\
\hline Q9CZU3 & Superkiller viralicidic activity 2-like 2 OS=Mus musculus GN=Skiv212 PE=1 SV=1 - [SK2L2_MOUSE] & $0,000 \mathrm{E} 0$ & $0,000 \mathrm{E} 0$ & $0,000 \mathrm{E} 0$ & $4,226 E 5$ \\
\hline Q9ESVO & ATP-dependent RNA helicase DDX24 OS=Mus musculus GN=Dd×24 PE=1 SV=2 - [DDX24_MOUSE] & $0,000 \mathrm{E} 0$ & $0,000 \mathrm{E} 0$ & $0,000 \mathrm{E} 0$ & 1,063E6 \\
\hline Q9D853 & ADP-ribosylation factor GTPase-activating protein 3 OS=Mus musculus $\mathrm{GN}=\mathrm{Arfgap} 3 \mathrm{PE}=1 \mathrm{SV}=2$ - [ARFG3_MOUSE] & $0,000 E_{0}$ & $0,000 \mathrm{E} 0$ & $0,000 E_{0}$ & $1,234 E 6$ \\
\hline Q70FJ1-3 & Isoform 3 of A-kinase anchor protein 9 OS=Mus musculus GN=Akap9 - [AKAP9_MOUSE] & 0,000 E & $0,000 \mathrm{E} 0$ & $0,0000_{0}$ & \begin{tabular}{l|l}
$1,105 E 6$ \\
\end{tabular} \\
\hline Q8BFY9-2 & Isoform 2 of Transportin-1 OS=Mus musculus GN=Tnpo1 - [TNPO1_MOUSE] & $0,000 \mathrm{E} 0$ & $0,000 \mathrm{E} 0$ & $0,000 \mathrm{E} 0$ & $2,208 \mathrm{E} 6$ \\
\hline Q8CJ96 & Ras association domain-containing protein 8 OS=Mus musculus GN=Rasf8 PE $=2 \mathrm{SV}=1$ - [RASF8_MOUSE] & $0,000 E 0$ & $0,000 E 0$ & $\begin{array}{r}, 000 E 0 \\
\end{array}$ & 3,567E6 \\
\hline
\end{tabular}




\begin{tabular}{|c|c|c|c|c|c|c|}
\hline Q920Q4-2 & Isoform 2 of Vacuolar protein sorting-associated protein 16 homolog OS=Mus musculus GN=Vps16 - [VPS16_MOUSE] & $0,000 E 0$ & $0,000 \mathrm{E} 0$ & $0,000 \mathrm{E} 0$ & $1,449 E 6$ & $2,497 E 5$ \\
\hline P97789-3 & Isoform 3 of 5'-3' exoribonuclease 1 OS=Mus musculus GN=Xrn1 - [XRN1_MOUSE] & $0,000 E 0$ & $0,000 E_{0}$ & $0,0000_{0}$ & $4,721 \mathrm{E}$ & $5,406 E 7$ \\
\hline P97798-5 & Isoform 5 of Neogenin OS=Mus musculus GN=Ne01 - [NEO1_MOUSE] & $0,000 E 0$ & $0,000 \mathrm{E} 0$ & $0,000 \mathrm{E} 0$ & 1,446E 6 & 4,804E6 \\
\hline Q88Z59 & $39 S$ ribosomal protein L48, mitochondrial OS=Mus musculus GN=Mrp|48 PE=1 SV=1 - [RM48_MOUSE] & $0,000 E 0$ & $0,000 E 0$ & $0,000 \mathrm{E} 0$ & $1,058 E 6$ & $2,448 E 6$ \\
\hline Q6PDHO & Pleckstrin homology-like domain family B member 1 OS=Mus musculus GN=Phldb1 PE=1 $\mathrm{SV}=1$ - [PHLB1_MOUSE] & $0,000 E 0$ & $0,000 E_{0}$ & $0,000 \mathrm{E} 0$ & $6,779 E 5$ & $2,258 \mathrm{E} 6$ \\
\hline Q8R418-2 & Isoform 2 of Endoribonuclease Dicer OS=Mus musculus GN=Dicer1 - [DICER_MOUSE] & $0,000 E_{0}$ & $0,000 \mathrm{E} 0$ & $0,000 \mathrm{E} 0$ & $1,087 E 6$ & 2,393E6 \\
\hline P58404-2 & Isoform 2 of Striatin-4 OS=Mus musculus GN=Strn4 - [STRN4_MOUSE] & $0,000 E 0$ & $0,000 E_{0}$ & 0,000E0 & $5,413 E 5$ & $2,563 \mathrm{EE}$ \\
\hline 054774 & AP-3 complex subunit delta-1 OS=Mus musculus GN=AP3d1 PE=1 SV=1 - [AP3D1_MOUSE] & $0,000 \mathrm{E} 0$ & $0,000 E_{0}$ & $0,000 \mathrm{E} 0$ & $3,698 \mathrm{E} 6$ & $7,873 E 5$ \\
\hline Q9WVL3 & Solute carrier family 12 member 7 OS=Mus musculus GN=SIc12a7 PE=1 SV=1 - [S12A7_MOUSE] & $0,000 E 0$ & $0,000 E_{0}$ & $0,0000_{0}$ & $3,468 E 6$ & $4,885 \mathrm{E} 6$ \\
\hline Q6PAR5-5 & Isoform 5 of GTPase-activating protein and VPS9 domain-containing protein 1 OS=Mus musculus GN=Gapvd1 - [GAPD1_MOUSE] & $0,000 \mathrm{E} 0$ & 0,000E0 & $0,000 \mathrm{E} 0$ & 1,895E6 & 3,290E6 \\
\hline Q8VE73-3 & Isoform 2 of Cullin-7 OS=Mus musculus GN=Cul7 - [CUL7_MOUSE] & $0,000 E 0$ & $0,000 \mathrm{E} 0$ & $0,000 \mathrm{E} 0$ & $1,794 \mathrm{E} 6$ & $2,435 \mathrm{E} 6$ \\
\hline Q6DFX2 & Anthrax toxin receptor $2 \mathrm{OS}=$ Mus musculus $\mathrm{GN}=\mathrm{Antx} \times 2$ PE $=1 \mathrm{SV}=1$ - [ANTR2_MOUSE] & $0,000 E 0$ & $0,000 E_{0}$ & $0,000 \mathrm{E}^{\circ}$ & $5,963 \mathrm{E} 6$ & 7,414E6 \\
\hline Q6P5E4 & UDP-glucose:glycoprotein glucosyltransferase $1 \mathrm{OS}=$ Mus musculus $\mathrm{GN}=$ Uggt1 PE=1 $\mathrm{SV}=4$ - [UGGG1_MOUSE] & $0,000 E 0$ & $0,000 E 0$ & $0,000 \mathrm{E} 0$ & $2,985 \mathrm{E} 6$ & 4,484E6 \\
\hline Q9DBB4 & N-alpha-acetyltransferase 16, NatA auxiliary subunit OS=Mus musculus GN=Naa16 PE=2 SV=1 - [NAA16_MOUSE] & $0,000 E 0$ & $0,000 E 0$ & $0,000 E_{0}$ & $4,245 E 6$ & $1,204 E 7$ \\
\hline Q6PDQ2 & Chromodomain-helicase-DNA-binding protein 4 OS=Mus musculus GN=Chd4 PE=1 SV=1 - [CHD4_MOUSE] & $0,000 E 0$ & $0,000 E_{0}$ & $0,000 \mathrm{E} 0$ & $5,787 \mathrm{E}$ & $4,604 \mathrm{E} 6$ \\
\hline Q8COE3 & Tripartite motif-containing protein $47 \mathrm{OS}=$ Mus musculus GN=Trim47 PE $=1 \mathrm{SV}=2-$ [TRI47_MOUSE] & $0,000 E 0$ & $0,000 \mathrm{E} 0$ & $0,000 E 0$ & $2,201 E 6$ & $1,115 \mathrm{E} 6$ \\
\hline Q99MD9-2 & Isoform 2 of Nuclear autoantigenic sperm protein OS=Mus musculus GN=Nasp - [NASP_MOUSE] & $0,000 E 0$ & $0,000 E_{0}$ & $0,000 \mathrm{E} 0$ & $1,443 E 6$ & $2,879 \mathrm{E} 6$ \\
\hline P06795 & Multidrug resistance protein $1 \mathrm{~B}$ OS=Mus musculus GN=Abcb1b PE=1 $\mathrm{SV}=1$ - [MDR1B_MOUSE] & $0,000 E 0$ & $0,000 E_{0}$ & $0,000 E 0$ & $8,525 E 5$ & $2,665 \mathrm{E} 6$ \\
\hline Q3UYV9 & Nuclear cap-binding protein subunit 1 OS=Mus musculus GN=Ncbp1 PE=1 SV=2 - [NCBP1_MOUSE] & $0,000 E 0$ & $0,000 E_{0}$ & $0,0000_{0}$ & $3,681 E 6$ & $5,270 \mathrm{E} 6$ \\
\hline A2AR02 & Peptidyl-prolyl cis-trans isomerase G OS=Mus musculus GN=Ppig PE=1 SV=1 - [PPIG_MOUSE] & $0,000 E 0$ & $0,000 \mathrm{E} 0$ & $0,000 \mathrm{E} 0$ & $3,317 \mathrm{E}$ & $5,165 \mathrm{E} 6$ \\
\hline P17809 & Solute carrier family 2, facilitated glucose transporter member 1 OS=Mus musculus GN=SIC2a1 PE=1 $\mathrm{SV}=4$ - [GTR1_MOUSE] & $0,000 E_{0}$ & $0,000 \mathrm{E} 0$ & $0,000 \mathrm{E} 0$ & $1,538 \mathrm{E} 6$ & $4,671 E 6$ \\
\hline Q75IG6 & Aff-GAP with SH3 domain, ANK repeat and PH domain-containing protein $2 \mathrm{OS}=$ Mus musculus $\mathrm{GN}=\mathrm{Asap2}$ PE=1 $\mathrm{SV}=3$ - [ASAP2_MOUSE] & $0,000 E 0$ & $0,000 \mathrm{E} 0$ & $0,000 E_{0}$ & $7,767 \pm 5$ & $3,134 \mathrm{E} 6$ \\
\hline Q91269 & SLTT-ROBO Rho GTPase-activating protein 1 OS=Mus musculus GN=Srgap1 PE=1 SV=2 - [SRGP1_MOUSE] & $0,0000_{0}$ & $0,000 \mathrm{E} 0$ & 0,000E0 & $1,599 \mathrm{EZ}$ & 2,463E7 \\
\hline Q640Q5 & PAB-dependent poly(A)-specific ribonuclease subunit PAN3 OS=Mus musculus GN=Pan3 PE=1 SV=3 - [PAN3_MOUSE] & $0,000 E 0$ & $0,000 E_{0}$ & $0,0000_{0}$ & $1,110 \mathrm{E} 6$ & $2,124 E 6$ \\
\hline Q8BUR4 & Dedicator of cytokinesis protein 1 OS=Mus musculus GN=Dock1 PE=1 SV=3 - [DOCK1_MOUSE] & $0,000 E_{0}$ & 0,000E0 & $0,000 \mathrm{E} 0$ & 1,558E6 & \begin{tabular}{l|l}
$4,658 E 6$ \\
\end{tabular} \\
\hline P18406 & Protein CYR61 OS=Mus musculus GN=Cyr61 PE=1 SV=1 - [CYR61_MOUSE] & $0,000 E 0$ & $0,000 E 0$ & $0,000 E 0$ & 8,36555 & $3,064 \mathrm{E} 6$ \\
\hline Q905V5 & Cullin-5 OS=Mus musculus GN=Cul5 PE=1 SV=3 - [CUL5_MOUSE] & $0,000 \mathrm{E} 0$ & $0,000 \mathrm{E} 0$ & $0,000 \mathrm{EO}$ & 6,775E6 & $2,137 E 6$ \\
\hline Q08297 & DNA repair protein RAD51 homolog 1 OS=Mus musculus GN=Rad51 PE=1 SV=1- [RAD51_MOUSE] & $0,000 E 0$ & $0,000 E_{0}$ & $0,000 \mathrm{E} 0$ & 5,634E5 & $3,666 \mathrm{E} 6$ \\
\hline Q9WTU6-2 & Isoform Alpha-1 of Mitogen-activated protein kinase 9 OS=Mus musculus GN=Mapk9 - [MK09_MOUSE] & $0,000 E 0$ & $0,000 E 0$ & $0,000 E 0$ & $4,305 E 6$ & $6,578 E 6$ \\
\hline Q9D742 & AP-5 complex subunit sigma-1 $\mathrm{OS}=$ =Mus musculus GN=Ap5s1 PE=1 SV=1 - [AP5S1_MOUSE] & $0,000 E 0$ & $0,000 E 0$ & $0,000 E 0$ & $1,281 E 7$ & $4,401 E 7$ \\
\hline P08122 & Collagen alpha-2(IV) chain OS=Mus musculus GN=COl4a2 PE=1 SV=4 - [CO4A2_MOUSE] & $0,000 \mathrm{E} 0$ & $0,000 E 0$ & $0,000 E 0$ & $8,132 E 5$ & $8,923 \mathrm{EE} 6$ \\
\hline Q14AX6-3 & Isoform 3 of Cyclin-dependent kinase 12 OS=Mus musculus GN=Cdk12 - [CDK12_MOUSE] & $0,000 E_{0}$ & $0,000 E 0$ & $0,000 \mathrm{E} 0$ & 7,773E6 & 1,847E7 \\
\hline P48722-2 & Isoform 2 of Heat shock $70 \mathrm{kDa}$ protein $4 \mathrm{LL}$ OS=Mus musculus GN=Hspa41 - [HS74L_MOUSE] & $0,000 \mathrm{E} 0$ & $0,000 E 0$ & $0,000 \mathrm{E} 0$ & 3,849E6 & $1,344 \mathrm{E}$ \\
\hline Q8K2Z4 & Condensin complex subunit 1 OS=Mus musculus GN=Ncapd2 PE=1 SV=2 - [CND1_MOUSE] & $0,000 E 0$ & $0,000 E_{0}$ & $0,000 E_{0}$ & $3,405 E 6$ & $4,844 \mathrm{EE}$ \\
\hline Q9D0G0 & $28 \mathrm{~S}$ ribosomal protein S30, mitochondrial OS=Mus musculus GN=Mrps30 PE=1 $\mathrm{SV}=1$ - [RT30_MOUSE] & $0,000 E 0$ & $0,000 \mathrm{E} 0$ & $0,0000_{0}$ & $1,736 \mathrm{E} 6$ & 6,170E6 \\
\hline Q9DBRO & A-kinase anchor protein 8 OS=Mus musculus GN=Akap8 PE=1 SV=1 - [AKAP8_MOUSE] & $0,000 E 0$ & $0,000 E 0$ & $0,000 \mathrm{E} 0$ & $4,310 \mathrm{E} 6$ & $2,684 E 6$ \\
\hline Q8K4R9-3 & Isoform 3 of Disks large-associated protein 5 OS=Mus musculus GN=Dlgap5 - [DLGP5_MOUSE] & $0,000 E_{0}$ & $0,000 E 0$ & $0,000 \mathrm{E} 0$ & $7,256 \mathrm{E}$ & 1,115E6 \\
\hline P11881-8 & Isoform 8 of Inositol 1,4,5-trisphosphate receptor type 1 OS=Mus musculus GN=Itpr1 - [ITPR1_MOUSE] & $0,000 E_{0}$ & $0,000 \mathrm{E} 0$ & $0,000 \mathrm{E} 0$ & $3,231 \mathrm{E} 6$ & 6,095E6 \\
\hline Q9DC23 & DnaJ homolog subfamily C member 10 OS=Mus musculus GN=Dnajc10 PE=1 SV=2 - [DJC10_MOUSE] & $0,000 \mathrm{E}$ & $0,000 \mathrm{E} 0$ & $0,000 \mathrm{E} 0$ & $8,297 E 5$ & 6,589E6 \\
\hline E9PZ8 & Activating signal cointegrator 1 complex subunit $3 \mathrm{OS}=$ Mus musculus $\mathrm{GN}=\mathrm{Ascc} 3 \mathrm{PE}=1 \mathrm{SV}=1$ - [ASCC3_MOUSE] & $0,000 \mathrm{E}_{0}$ & 0,000E0 & 0,000E0 & 1,845E6 & 3,609E6 \\
\hline Q99KQ4 & Nicotinamide phosphoribosyltransferase $\mathrm{OS}=$ Mus musculus $\mathrm{GN}=\mathrm{Nampt} \mathrm{PE}=1 \mathrm{SV}=1$ - [NAMPT_MOUSE] & $0,000 E 0$ & $0,000 E 0$ & $0,000 E 0$ & 1,363E6 & $7,218 \mathrm{E} 5$ \\
\hline P33174 & Chromosome-associated kinesin KIF4 OS=Mus musculus GN=Kif4 PE=1 SV=3 - [KIF4_MOUSE] & $0,000 E 0$ & $0,000 E_{0}$ & $0,000 \mathrm{E} 0$ & 1,563E6 & $2,604 E 6$ \\
\hline Q8BQN6 & Zinc finger protein OZF OS=Mus musculus GN=Znf146 PE=2 SV=2 - [OZF_MOUSE] & $0,000 \mathrm{E} 0$ & $0,000 E 0$ & $0,000 E 0$ & $1,770 \mathrm{E} 6$ & $5,388 \mathrm{EE} 6$ \\
\hline P52431 & DNA polymerase delta catalytic subunit OS=Mus musculus GN=Pold1 $1 \mathrm{PE}=1 \mathrm{SV}=2$ - [DPOD1_MOUSE] & $0,000 \mathrm{E}_{0}$ & $0,000 E 0$ & $0,000 \mathrm{E} 0$ & $5,264 E 5$ & 2,011E6 \\
\hline Q9QXD8 & LIM domain-containing protein 1 OS=Mus musculus GN=Limd1 PE=1 SV=2 - [LMD1_MOUSE] & $0,000 \mathrm{E}_{0}$ & 0,000E0 & $0,000 \mathrm{E} 0$ & $1,322 \mathrm{E} 6$ & \begin{tabular}{l|l}
$5,519 E 6$ \\
\end{tabular} \\
\hline P15306 & Thrombomodulin OS=Mus musculus GN=Thbd PE=1 SV=1 - [TRBM_MOUSE] & $0,000 E 0$ & $0,000 E_{0}$ & $0,000 E_{0}$ & $3,583 \mathrm{E} 7$ & $1,059 \mathrm{E} 7$ \\
\hline Q8CDJ8 & Stonin-1 OS=Mus musculus GN=Ston1 PE=1 SV=2 - [STON1_MOUSE] & $0,000 E 0$ & $0,000 \mathrm{E} 0$ & $0,000 \mathrm{EO}$ & $1,355 \mathrm{EE}$ & 3,264E6 \\
\hline P42225 & Signal transducer and activator of transcription 1 OS=Mus musculus $\mathrm{GN}=\mathrm{Stat1} \mathrm{PE}=1 \mathrm{SV}=1$ - [STAT1_MOUSE] & $0,000 E_{0}$ & 0,000E0 & $0,000 \mathrm{E} 0$ & 1,240E6 & $8,767,5$ \\
\hline Q92329-3 & Isoform 3 of Inositol 1,4,5-trisphosphate receptor type 2 OS=Mus musculus GN=Itpr2 - [ITPR2_MOUSE] & $0,000 E 0$ & $0,000 E_{0}$ & $0,000 \mathrm{E} 0$ & $3,667 \mathrm{E}$ & 4,245E6 \\
\hline Q99PV0 & Pre-mRNA-processing-splicing factor 8 OS=Mus musculus GN=Prpf8 PE=1 SV=2 - [PRP8_MOUSE] & $0,000 E 0$ & $0,000 E_{0}$ & $0,000 E 0$ & $8,824 E 5$ & $9,027 E 5$ \\
\hline Q8BFZ2-2 & Isoform 2 of Phospholipid phosphatase-related protein type 1 OS=Mus musculus GN=Plppr1 - [PLPR1_MOUSE] & $0,000 \mathrm{E} 0$ & $0,000 E 0$ & $0,0000_{0}$ & $5,935 \mathrm{E} 6$ & 9,569E6 \\
\hline Q99JW4 & LIM and senescent cell antigen-like-containing domain protein 1 OS=Mus musculus GN=Lims1 PE=1 SV=3 - [LIMS1_MOUSE] & $0,000 \mathrm{E} 0$ & $0,000 \mathrm{E} 0$ & $0,000 \mathrm{E}$ & 5,696E6 & \begin{tabular}{l|l}
$9,240 \mathrm{E} 6$ \\
\end{tabular} \\
\hline Q7TPD1-3 & Isoform 3 of F-box only protein 11 OS=Mus musculus GN=Fbxo11 - [FBX11_MOUSE] & $0,000 E 0$ & $0,000 E 0$ & $0,000 \mathrm{E} 0$ & 6,60955 & $2,284 E 6$ \\
\hline Q9DCC4 & Pyrroline-5-carboxylate reductase 3 OS=Mus musculus GN=Pycrl PE=1 SV=2 - [P5CR3_MOUSE] & $0,000 E 0$ & $0,000 E_{0}$ & $0,000 E 0$ & $1,854 E 6$ & $1,452 E 6$ \\
\hline Q9R1S8 & Calpain-7 OS=Mus musculus GN=Capn7 PE=2 SV=1 - [CAN7_MOUSE] & $0,000 \mathrm{E} 0$ & $0,000 \mathrm{E} 0$ & $0,000 E 0$ & $1,762 \mathrm{E} 6$ & $2,080 \mathrm{E} 6$ \\
\hline Q9WTR2 & Mitogen-activated protein kinase kinase kinase 6 OS=Mus musculus GN=Map3k6 PE=1 SV=4- [M3K6_MOUSE] & $0,000 E 0$ & $0,000 E_{0}$ & $0,000 \mathrm{E} 0$ & 7,774E8 & $1,237 \mathrm{Eg}$ \\
\hline Q9QYנ3 & Dna] homolog subfamily B member 1 OS=Mus musculus GN=Dnajb1 PE=1 1 SV=3 - [DNJB1_MOUSE] & $0,000 \mathrm{E}_{0}$ & 0,000E0 & 0,000E0 & $2,528 \mathrm{E} 6$ & 1,835E6 \\
\hline Q71LX4 & Talin-2 OS=Mus musculus GN=TIn2 PE=1 SV=3 - [TLN2_MOUSE] & $0,000 E 0$ & $0,000 E 0$ & $0,000 E 0$ & $3,980 \mathrm{E} 6$ & $1,115 \mathrm{EF}$ \\
\hline F8VPU2 & FERM, RhoGEF and pleckstrin domain-containing protein 1 OS=Mus musculus GN=Farp1 PE=1SV=1- [FARP1_MOUSE] & $0,000 \mathrm{E} 0$ & $0,000 E 0$ & $0,0000_{0}$ & 6,273E5 & 1,829E6 \\
\hline P83741-4 & Isoform 4 of Serine/threonine-protein kinase WNK1 OS=Mus musculus GN=Wnk1 - [WNK1_MOUSE] & $0,000 \mathrm{EO}$ & $0,000 \mathrm{E} 0$ & $0,000 \mathrm{E} 0$ & 1,033E6 & $1,671 \mathrm{E} 6$ \\
\hline P70206 & Plexin-A1 OS=Mus musculus GN=Plxna1 PE=1 SV=1 - [PLXA1_MOUSE] & $0,000 E 0$ & $0,000 E_{0}$ & $0,000 \mathrm{E} 0$ & $2,637 \mathrm{E}$ & 3,080E6 \\
\hline P53995 & Anaphase-promoting complex subunit 1 OS=Mus musculus GN=Anapc1 PE=1 SV=2 - [APC1_MOUSE] & $0,000 E 0$ & $0,000 E 0$ & $0,000 \mathrm{E} 0$ & $2,948 E 6$ & $2,756 \mathrm{EE}$ \\
\hline Q9Q773 & Transmembrane protein 59 OS=Mus musculus GN=Tmem59 PE=1 SV=2 - [TMM59_MOUSE] & $0,000 \mathrm{E} 0$ & $0,000 \mathrm{E} 0$ & $0,000 E 0$ & $8,690 \mathrm{E} 6$ & 5,998E6 \\
\hline P00532 & Serine/threonine-protein kinase-transforming protein raf OS=Murine sarcoma virus $3611 \mathrm{GN}=\mathrm{V}-\mathrm{RAF}$ PE=3 SV=1 - [RAF_MSV36] & $0,000 E 0$ & $0,000 E_{0}$ & $0,000 \mathrm{E} 0$ & $3,362 E 6$ & $6,219 \mathrm{E} 6$ \\
\hline Q9נIS8 & Solute carrier family 12 member 4 OS=Mus musculus $\mathrm{GN}=S \mathrm{SI} 12 \mathrm{a} 4 \mathrm{PE}=1 \mathrm{SV}=2$ - [S12A4_MOUSE] & $0,000 E 0$ & $0,000 E 0$ & $0,000 E 0$ & $2,203 E 6$ & 7,992E6 \\
\hline Q6P549 & Phosphatidylinositol 3,4,5-trisphosphate 5-phosphatase 2 OS=Mus musculus GN=Inppl1 PE=1 SV=1 - [SHIP2_MOUSE] & $0,000 E 0$ & $0,000 E_{0}$ & $0,000 \mathrm{E} 0$ & 4,295E6 & 6,776E6 \\
\hline Q5SSK3 & Transcription elongation factor, mitochondrial OS=Mus musculus GN=Tefm PE=1 SV=1 - [TEFM_MOUSE] & $0,000 E_{0}$ & $0,000 \mathrm{E} 0$ & $0,0000_{0}$ & $5,918 E 5$ & $1,960 \mathrm{E} 6$ \\
\hline Q9Z0R4 & Intersectin-1 OS=Mus musculus GN=Itsn1 PE=1 SV=2 - [ITSN1_MOUSE] & $0,000 \mathrm{EO}_{0}$ & $0,000 \mathrm{E} 0$ & $0,000 \mathrm{E} 0$ & $4,169 \mathrm{E} 6$ & $2,358 \mathrm{E} 6$ \\
\hline D3YXK2 & Scaffold attachment factor B1 OS=Mus musculus GN=Safb PE=1 SV=2 - [SAFB1_MOUSE] & $0,000 E_{0}$ & 0,000E0 & 0,000E0 & $2,573 \mathrm{E} 6$ & \begin{tabular}{l|l}
$5,010 \mathrm{E} 6$ \\
\end{tabular} \\
\hline P55937-1 & Isoform 1 of Golgin subfamily A member 3 OS=Mus musculus GN=Golga3 - [GOGA3_MOUSE] & $0,000 E 0$ & $0,000 E 0$ & $0,000 \mathrm{E} 0$ & $1,080 \mathrm{E} 6$ & $1,569 \mathrm{EE}$ \\
\hline Q97280-2 & Isoform PLD1B of Phospholipase D1 OS=Mus musculus GN=Pld1 - [PLD1_MOUSE] & $0,000 E 0$ & $0,000 E_{0}$ & $0,000 E_{0}$ & $2,111 \mathrm{E} 6$ & $2,099 \mathrm{E} 6$ \\
\hline BAAVY7 & Kinesin-like protein KIF16B OS=Mus musculus GN=Kif16b PE=1 SV=1 - [KI16B_MOUSE] & $0,000 \mathrm{E} 0$ & $0,000 \mathrm{E} 0$ & $0,000 \mathrm{E} 0$ & 1,563E6 & $2,138 \mathrm{E} 6$ \\
\hline Q4JIM5 & Abelson tyrosine-protein kinase 2 OS=Mus musculus GN=Ab12 PE=1 SV=1 - [ABL2_MOUSE] & $0,000 E 0$ & $0,000 E_{0}$ & $0,000 E_{0}$ & $1,355 \mathrm{E} 6$ & $1,597 \mathrm{E} 6$ \\
\hline Q9R117 & Non-receptor tyrosine-protein kinase TYK2 OS=Mus musculus GN=Tyk2 PE=1 SV=2 - [TYK2_MOUSE] & $0,000 E 0$ & $0,000 E_{0}$ & $0,000 E_{0}$ & $2,786 \mathrm{E} 6$ & $6,157 \mathrm{E} 6$ \\
\hline Q8BWY9 & Protein CIP2A OS=Mus musculus GN=Kiaa1524 PE=1 SV=3 - [CIP2A_MOUSE] & $0,000 \mathrm{E} 0$ & $0,000 E 0$ & $0,000 E 0$ & $1,554 \mathrm{E} 6$ & 2,989E6 \\
\hline $088738-3$ & Isoform 3 of Baculoviral IAP repeat-containing protein 6 OS=Mus musculus GN=Birc6 - [BIRC6_MOUSE] & $0,000 E 0$ & $0,000 E_{0}$ & $0,000 \mathrm{E} 0$ & 5,657E6 & $4,645 E 6$ \\
\hline Q80TV8-2 & Isoform 2 of CLIP-associating protein 1 OS=Mus musculus GN=Clasp1 - [CLAP1_MOUSE] & $0,000 E 0$ & $0,000 \mathrm{E} 0$ & $0,000 \mathrm{E} 0$ & $1,014 E 6$ & $1,426 \mathrm{E} 6$ \\
\hline P59913 & Protein-L-isoaspartate O-methyltransferase domain-containing protein 1 OS=Mus musculus GN=Pcmtd1 PE=1 SV=1 - [PCMD1_MOUSE] & $0,000 \mathrm{E} 0$ & $0,000 E_{0}$ & $0,000 E_{0}$ & $3,097 \mathrm{E}$ & $2,367 \mathrm{E} 6$ \\
\hline P01872 & Ig mu chain C region OS=Mus musculus GN=Ighm PE=1 SV=2 - [IGHM_MOUSE] & $0,000 E 0$ & $0,000 E_{0}$ & $0,000 E_{0}$ & $4,839 \mathrm{E} 7$ & $4,957 \mathrm{E} 8$ \\
\hline Q99M51 & Cytoplasmic protein NCK1 OS=Mus musculus GN=Nck1 PE=1 SV=1 - [NCK1_MOUSE] & $0,000 E 0$ & $0,000 \mathrm{E} 0$ & $0,000 \mathrm{E} 0$ & $1,839 \mathrm{E} 6$ & 1,213E6 \\
\hline P35917 & Vascular endothelial growth factor receptor 3 OS=Mus musculus GN=Flt4 PE=1 SV=1 - [VGFR3_MOUSE] & $0,000 E 0$ & $0,000 E_{0}$ & $0,000 \mathrm{E}_{0}$ & $3,523 \mathrm{E} 6$ & $1,319 \mathrm{EF}$ \\
\hline Q8CGY8-2 & Isoform 2 of UDP-N-acetylglucosamine--peptide N-acetylglucosaminyltransferase $110 \mathrm{kDa}$ subunit OS=Mus musculus GN=Ogt - [OGT1_MOUSE] & $0,000 E 0$ & $0,000 E 0$ & $0,000 \mathrm{E} 0$ & $1,460 \mathrm{E} 6$ & 3,095E6 \\
\hline Q8VDG3 & Poly(A)-specific ribonuclease PARN OS=Mus musculus GN=Parn PE=1 SV=1 - [PARN_MOUSE] & $0,000 \mathrm{EO}_{0}$ & $0,000 \mathrm{E} 0$ & $0,000 \mathrm{E} 0$ & $1,275 \mathrm{E} 6$ & $3,004 E 6$ \\
\hline Q7TMY8-4 & Isoform 4 of E3 ubiquitin-protein ligase HUWE1 OS=Mus musculus GN=Huwe1 - [HUWE1_MOUSE] & $0,000 E 0$ & $0,000 \mathrm{E} 0$ & $0,000 \mathrm{E} 0$ & $2,401 E 6$ & $1,430 \mathrm{E} 6$ \\
\hline Q3U214 & Microtubule-associated serine/threonine-protein kinase 3 OS=Mus musculus GN=Mast3 PE=1 SV=3 - [MAST3_MOUSE] & $0,000 \mathrm{E} 0$ & $0,000 E_{0}$ & $0,000 \mathrm{E}^{\circ}$ & $3,096 \mathrm{E} 6$ & $2,839 \mathrm{E} 6$ \\
\hline Q640M1 & U3 small nucleolar RNA-associated protein 14 homolog A OS=Mus musculus GN=Utp14a PE=1 SV=1 - [UT14A_MOUSE] & $0,000 E 0$ & $0,000 E_{0}$ & $0,0000_{0}$ & $1,773 \mathrm{E} 6$ & $8,582 E 5$ \\
\hline Q8BZQ7 & Anaphase-promoting complex subunit 2 OS=Mus musculus GN=Anapc2 PE=1 SV=2 - [ANC2_MOUSE] & $0,000 E 0$ & $0,000 E 0$ & $0,000 \mathrm{E} 0$ & 6,631E6 & $8,064 E 6$ \\
\hline A2AN08-3 & Isoform 3 of E3 ubiquitin-protein ligase UBR4 OS=Mus musculus GN=Ubr4 - [UBR4_MOUSE] & $0,000 E 0$ & $0,000 E_{0}$ & $0,000 \mathrm{E} 0$ & $2,192 E 6$ & $3,358 \mathrm{E} 6$ \\
\hline Q99MR1 & PERQ amino acid-rich with GYF domain-containing protein 1 OS=Mus musculus GN=Gigyf1 PE=1 SV=2 - [PERQ1_MOUSE] & $0,000 E 0$ & $0,000 \mathrm{E} 0$ & $0,000 E 0$ & $2,615 E 6$ & $5,911 \mathrm{E} 6$ \\
\hline Q91ZX7 & Prolow-density lipoprotein receptor-related protein 1 OS=Mus musculus GN=Lrp1 PE=1 SV=1- [LRP1_MOUSE] & $0,000 E 0$ & $0,000 E_{0}$ & $0,000 E_{0}$ & $2,824 E 6$ & $7,341 \mathrm{E} 6$ \\
\hline Q80WQ6 & Inactive rhomboid protein $2 \mathrm{OS}=$ Mus musculus GN=Rhbdf2 PE=1 SV=1 - [RHDF2_MOUSE] & $0,000 \mathrm{E}$ & $0,000 E 0$ & 0,000E0 & $2,015 \mathrm{E} 6$ & $5,360 \mathrm{E} 6$ \\
\hline A2ASS6 & Titin OS=Mus musculus GN=Ttn PE=1 SV=1 - [TITIN_MOUSE] & $0,000 E 0$ & $0,000 \mathrm{E} 0$ & $0,000 \mathrm{E} 0$ & $5,187 \mathrm{E}$ & $8,800 \mathrm{E} 6$ \\
\hline
\end{tabular}




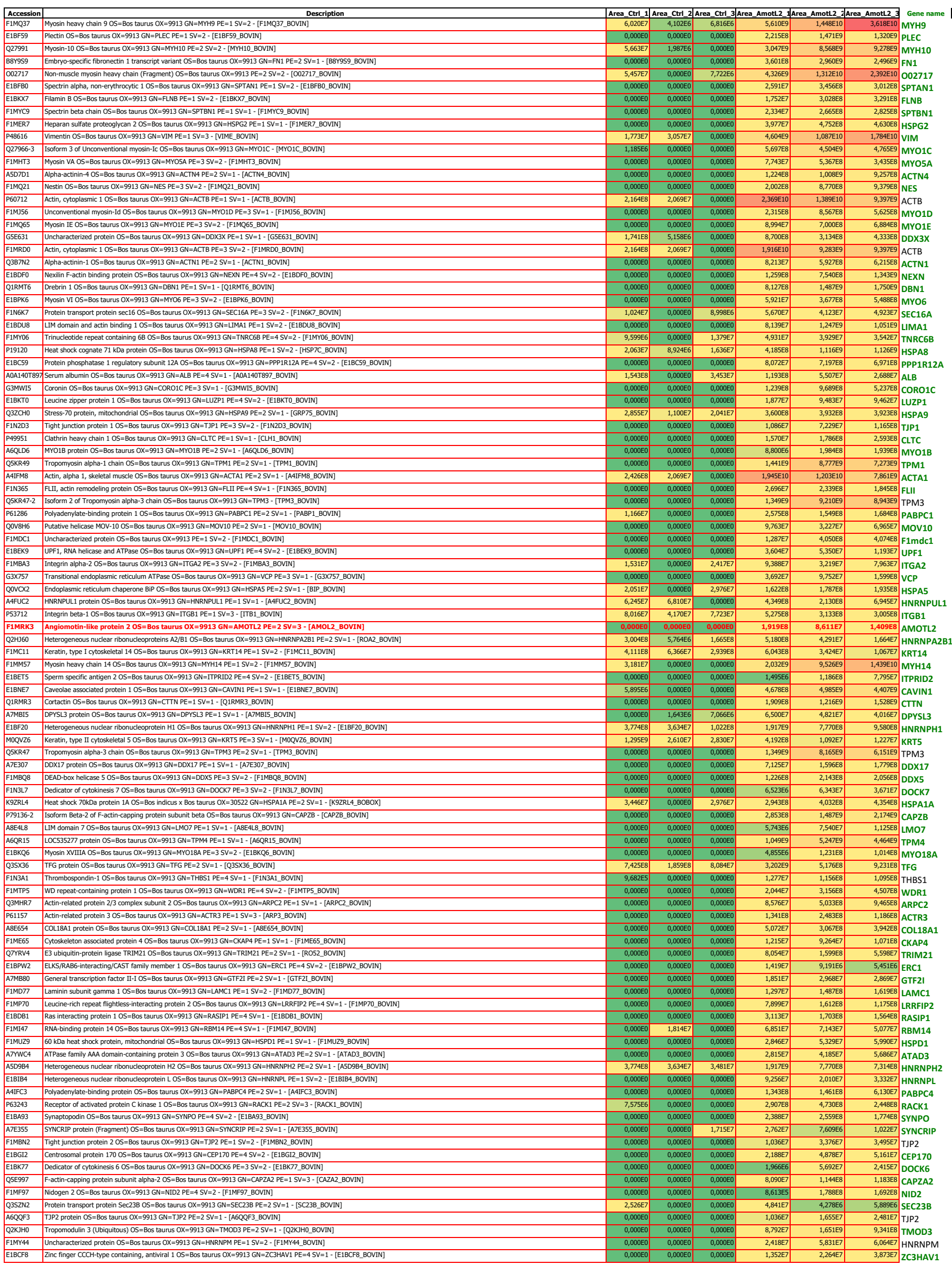




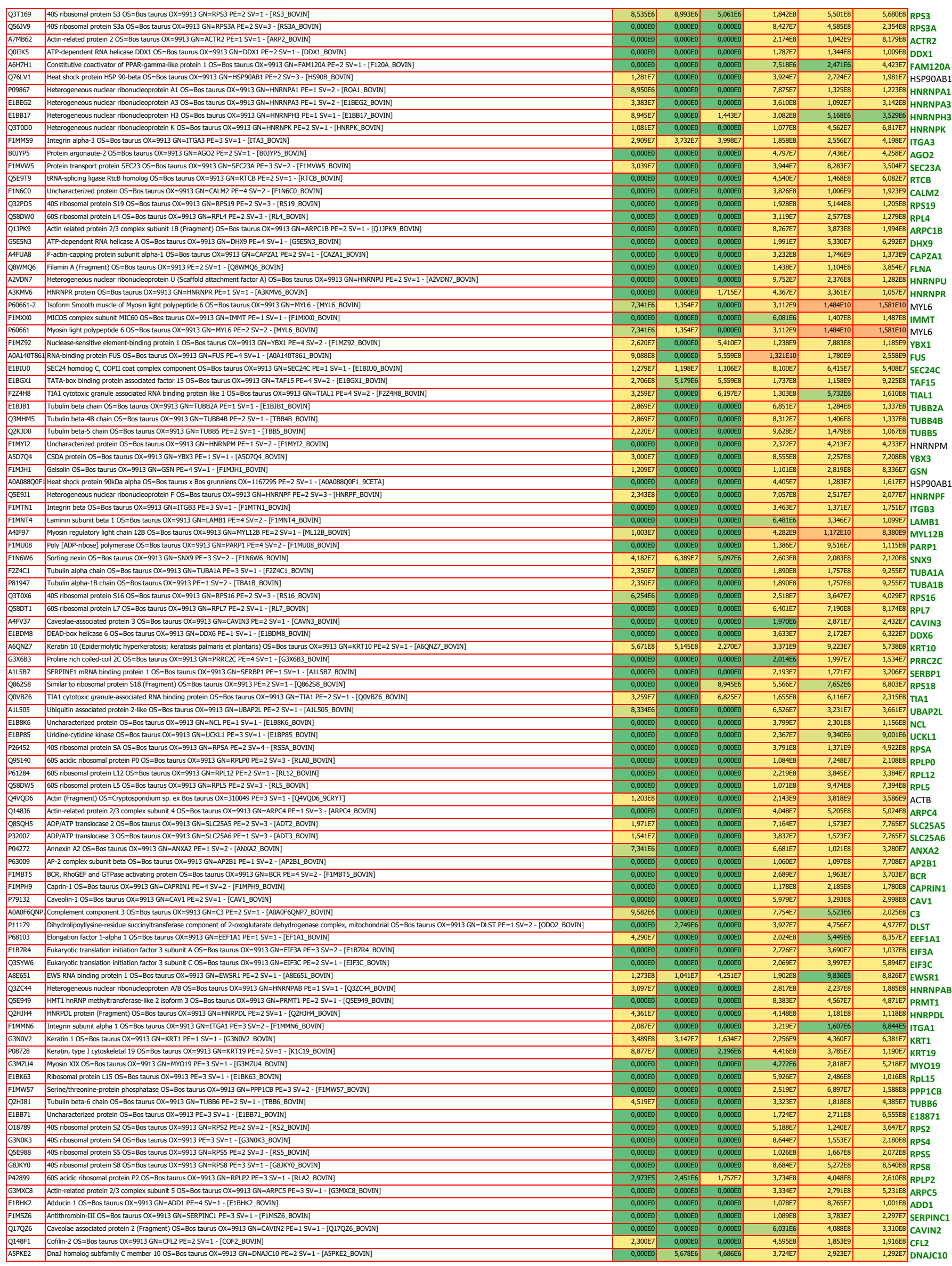




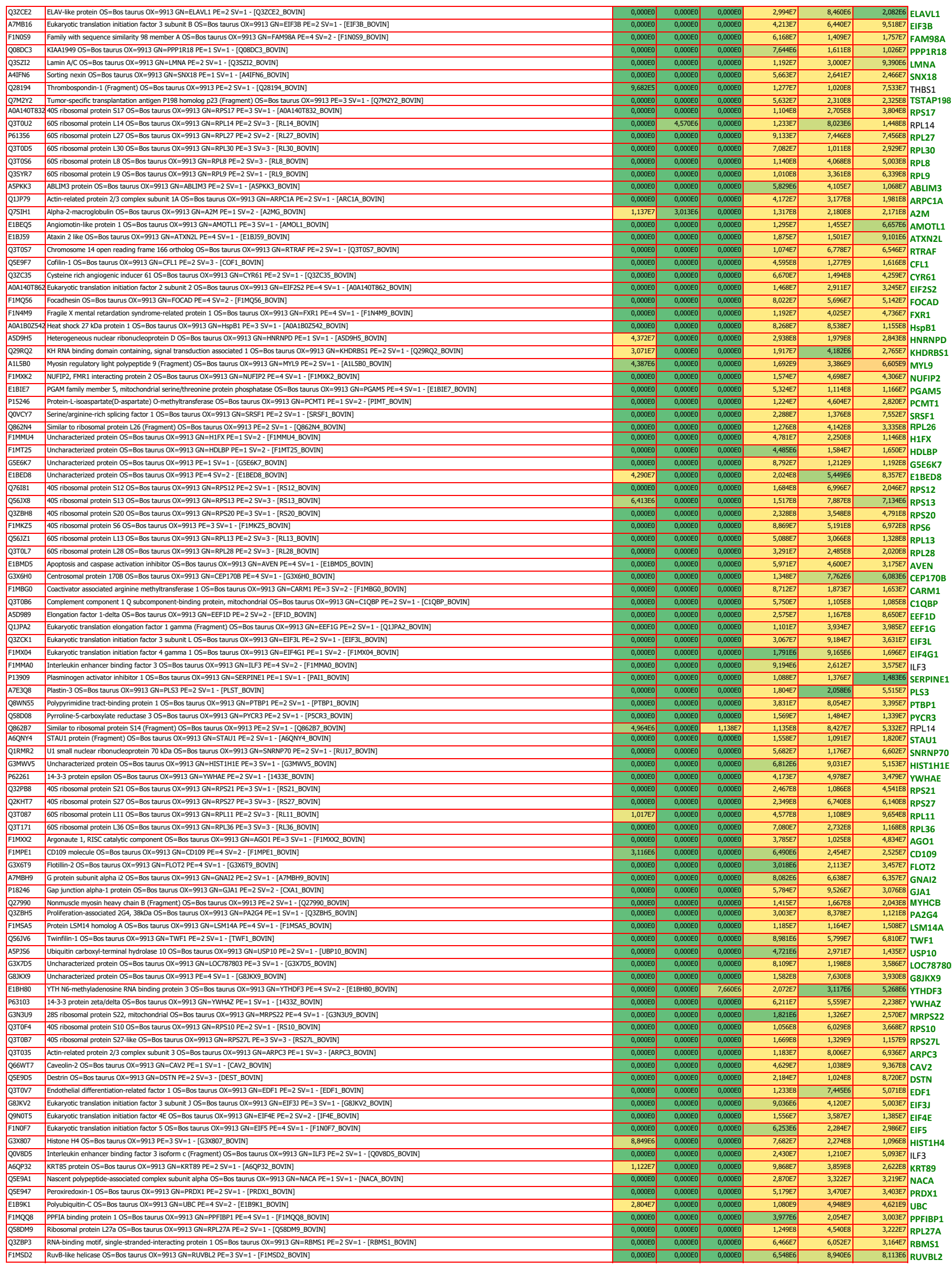




\begin{tabular}{|c|c|c|c|c|c|c|c|c|}
\hline Q35ZR8 & Serine/arginine-rich splicing factor $3 \mathrm{OS}=$ Bos taurus OX=9913 GN=SRSF3 PE=2 SV=1 - [SRSF3_BOVIN] & $0,000 \mathrm{E}^{\circ}$ & $0,000 \mathrm{E} 0$ & $0,000 \mathrm{E} 0$ & $1,704 \mathrm{E} 7$ & $4,621 \mathrm{E} 7$ & 4,985E6 & SRSF3 \\
\hline Q862R3 & Similar to ribosomal protein L18a (Fragment) OS=Bos taurus OX=9913 PE=2 SV=1 - [Q862R3_BOVIN] & $0,000 E 0$ & $0,000 \mathrm{E} 0$ & $0,000 \mathrm{E} 0$ & $3,525 \mathrm{E} 6$ & $1,486 E 8$ & $5,021 E 7$ & RPL18 \\
\hline F1MZ00 & Small nuclear ribonucleoprotein Sm D3 OS=Bos taurus OX=9913 GN=SNRPD3 PE=3 SV=2 - [F1MZOO_BOVIN] & $0,000 \mathrm{E}_{0}$ & $0,000 \mathrm{E} 0$ & $0,000 \mathrm{E} 0$ & $5,805 E 6$ & 9,885E7 & $5,016 \mathrm{E} 7$ & SNRPD3 \\
\hline E1BQ37 & Splicing factor proline and glutamine rich OS=Bos taurus OX=9913 GN=SFPQ PE=1 SV=1 - [E1BQ37_BOVIN] & $0,000 E_{0}$ & $0,000 \mathrm{EO}$ & $0,000 \mathrm{E} 0$ & $1,487 E 6$ & $1,750 E 6$ & $2,444 E 6$ & SFPQ \\
\hline AGH7J7 & Uncharacterized protein OS=Bos taurus OX=9913 PE=2 SV=1 - [A6H7J7_BOVIN] & $1,894 E 8$ & $0,000 \mathrm{E} 0$ & $0,000 \mathrm{E} 0$ & $2,272 E 8$ & 8,787E7 & $1,517 E 8$ & A6H7J7 \\
\hline Q56K10 & 40S ribosomal protein $\mathrm{S15}$ OS=Bos taurus OX=9913 GN=RPS15 PE=2 SV=3 - [RS15_BOVIN] & $0,000 \mathrm{E}_{0}$ & $0,000 \mathrm{E} 0$ & $0,000 \mathrm{E} 0$ & $1,377 E 8$ & $5,099 \mathrm{E}$ & $3,286 E_{8}$ & RPS15 \\
\hline Q35ZQ6 & 60S ribosomal protein L32 OS=Bos taurus OX=9913 GN=RPL32 PE=2 SV=3 - [RL132_BOVIN] & $0,000 \mathrm{E}_{0}$ & $0,000 \mathrm{EO}$ & $0,000 \mathrm{E} 0$ & $5,706 \mathrm{E}$ & $9,771 \mathrm{E}$ & 6,501E7 & RPL32 \\
\hline G3N2R1 & 60S ribosomal protein L6 OS=Bos taurus OX=9913 PE=3 SV=1 - [G3N2R1_BOVIN] & $0,000 E_{0}$ & $0,000 E 0$ & $0,000 \mathrm{E} 0$ & $1,007 E 8$ & 4,719E7 & $4,830 \mathrm{E}$ & RPL6 \\
\hline F1MLB8 & ATP synthase subunit alpha OS=Bos taurus OX=9913 GN=ATP5F1A PE=1 SV=1 - [F1MLB8_BOVIN] & $0,000 E 0$ & $0,000 E 0$ & $0,000 \mathrm{E} 0$ & $6,084 E 6$ & $1,694 \mathrm{E} 7$ & $6,376 \mathrm{E} 6$ & ATP5F1A \\
\hline Q32L.92 & Calponin-3 OS=Bos taurus OX=9913 GN=CNN3 PE=2 SV=1 - [CNN3_BOVIN] & $0,000 E_{0}$ & $0,000 \mathrm{E} 0$ & $0,000 \mathrm{E} 0$ & $6,621 E 6$ & $1,696 \mathrm{E} 6$ & $2,887 \mathrm{E}$ & CNN3 \\
\hline Q3ZBH3 & CD151 antigen OS=Bos taurus OX=9913 GN=CD151 PE=2 SV=1 - [CD151_BOVIN] & $1,829 \mathrm{E} 7$ & $0,000 \mathrm{E} 0$ & $0,000 \mathrm{E} 0$ & $2,437 E 8$ & $1,726 E 8$ & $1,498 \mathrm{~EB}$ & CD151 \\
\hline Q2KII4 & Elongin-C OS=Bos taurus OX=9913 GN=ELOC PE=3 SV=1 - [ELOC_BOVIN] & $1,731 E 6$ & $0,000 \mathrm{EO}$ & $0,000 \mathrm{E} 0$ & $2,217 \mathrm{E}$ & 4,299E7 & $5,625 \mathrm{E} 7$ & ELOC \\
\hline E1BLZ8 & Eukaryotic translation initiation factor 3 subunit F OS=Bos taurus OX=9913 GN=EIF3F PE=1 SV=2 - [E1BLZ8_BOVIN] & $0,000 E_{0}$ & $0,000 \mathrm{EO}$ & $0,000 \mathrm{E} 0$ & $3,096 \mathrm{E}$ & $1,714 E 6$ & $4,833 \mathrm{E} 7$ & EIF3F \\
\hline \begin{tabular}{|l|l|l} 
ASPK49 \\
\end{tabular} & IGL@ protein OS=Bos taurus OX=9913 GN=IGL@ PE=2 SV=1 - [ASPK49_BOVIN] & $5,852 E 8$ & 1,48957 & $6,150 \mathrm{E} 6$ & $1,867 \mathrm{~F}$ & $1,979 \mathrm{Eg}$ & $1,824 \mathrm{Eg}$ & RBM8A \\
\hline Q3ZCE8-2 & Isoform 2 of RNA-binding protein 8A OS=Bos taurus OX=9913 GN=RBM8A - [RBM8A_BOVIN] & $0,000 E_{0}$ & $0,000 \mathrm{E} 0$ & $0,000 \mathrm{E} 0$ & $8,167 \pm 6$ & $4,351 \mathrm{E} 6$ & $8,486 \mathrm{E} 6$ & RBM8A \\
\hline P00974 & Pancreatic trypsin inhibitor OS=Bos taurus OX=9913 PE=1 SV=2 - [BPT1_BOVIN] & $0,000 \mathrm{E} 0$ & $0,000 \mathrm{E} 0$ & $3,765 E 7$ & $1,766 \mathrm{E} 9$ & 7,076E8 & $2,062 E 8$ & BPT1 \\
\hline A5D9D7 & Procollagen-lysine, 2-oxoglutarate 5-dioxygenase 3 (Fragment) OS=Bos taurus OX=9913 GN=PLOD3 PE=2 SV=1 - [A5D9D7_BOVIN] & $0,000 E 0$ & $0,000 \mathrm{E} 0$ & $0,000 \mathrm{E} 0$ & $4,242 E 6$ & $5,760 E 6$ & $9,798 \mathrm{E}$ & PLOD3 \\
\hline QOVCF9 & Protein LSM12 homolog OS=Bos taurus OX=9913 GN=LSM12 PE=2 SV=2 - [LSM12_BOVIN] & $0,000 \mathrm{E}_{0}$ & $0,000 \mathrm{EO}$ & $0,000 \mathrm{E} 0$ & $2,548 \mathrm{E} 7$ & 4,155E7 & 5,952E7 & LSM12 \\
\hline F1N301 & Uncharacterized protein OS=Bos taurus OX=9913 GN=RPL22 PE=1 SV=2 - [F1N301_BOVIN] & $0,000 E 0$ & $0,000 E_{0}$ & $0,000 \mathrm{E} 0$ & $1,154 E 8$ & $2,741 E 8$ & $6,289 \mathrm{~EB}$ & RPL22 \\
\hline E1BHM9 & Uncharacterized protein OS=Bos taurus OX=9913 PE=3 SV=1 - [E1BHM9_BOVIN] & $0,000 \mathrm{E} 0$ & $0,000 \mathrm{EO}$ & $0,000 \mathrm{E} 0$ & $5,423 E 6$ & 1,843E8 & 7,843E7 & E18HM9 \\
\hline Q2K149 & 39 r ribosomal protein L50, mitochondrial OS=Bos taurus OX=9913 GN=MRPL50 PE=1 SV=1- [RM50_BOVIN] & $0,000 \mathrm{E}_{0}$ & $0,000 \mathrm{E} 0$ & $0,000 \mathrm{E} 0$ & $1,004 E 7$ & 6,539E6 & $5,574 E 6$ & MRPL50 \\
\hline Q58DW3 & 60 ribosomal protein L29 OS=Bos taurus OX=9913 GN=RPL29 PE=2 SV=3 - [RLL29BOVIN] & $0,000 \mathrm{EO}$ & $0,000 \mathrm{EO}$ & $0,000 \mathrm{E} 0$ & $6,262 \mathrm{E}$ & $1,076 E 8$ & 8,77677 & RPL29 \\
\hline P04973-2 & Isoform Non-brain of Clathrin light chain A OS=Bos taurus OX=9913 GN=CLTA - [CLCA_BOVIN] & $0,000 \mathrm{EO}$ & $0,000 \mathrm{E} 0$ & $0,000 \mathrm{E} 0$ & $1,010 E 7$ & $1,430 \mathrm{E} 8$ & $1,471 \mathrm{E} 8$ & CLTA \\
\hline F1MKI2 & RAB12, member RAS oncogene family OS=Bos taurus OX=9913 GN=RAB12 PE=4 SV=2 - [F1MKI2_BOVIN] & $0,000 E 0$ & $0,000 E 0$ & $0,000 \mathrm{E} 0$ & $3,911 \mathrm{E}$ & $2,651 \mathrm{E} 7$ & $1,653 \mathrm{E}$ & RAB12 \\
\hline QOVCQ9 & Reticulocalbin 2, EF-hand calcium binding domain OS=Bos taurus OX=9913 GN=RCN2 PE=2 SV=1 - [Q0VCQ9_BOVIN] & $0,000 \mathrm{E} 0$ & $0,000 \mathrm{E} 0$ & $0,000 \mathrm{E} 0$ & 3,154E6 & $1,860 \mathrm{E} 7$ & $2,527=6$ & RCN2 \\
\hline Q32PA0 & U1 small nuclear ribonucleoprotein C OS=Bos taurus OX=9913 GN=SNRPC PE=2 SV=2 - [RU1C_BOVIN] & $0,000 \mathrm{EO}^{2}$ & $0,000 E 0$ & $0,000 \mathrm{E} 0$ & $8,697 \mathrm{E}$ & $1,857 \mathrm{E}$ & $4,872 E 6$ & SNRPC \\
\hline F1MI18 & Uncharacterized protein OS=Bos taurus OX=9913 PE=4 SV=2 - [F1MI18_BOVIN] & $0,000 E 0$ & $0,000 E_{0}$ & $0,000 \mathrm{E} 0$ & $1,437 E 8$ & $2,459 \mathrm{E}$ & 3,093E8 & F1Ml18 \\
\hline
\end{tabular}


Gene_ID

ENSMUSG00000000290

ENSMUSG000000002111

ENSMUSG00000003032

ENSMUSG00000005338

ENSMUSG00000010064

ENSMUSG00000010797

ENSMUSG00000012705

ENSMUSG00000015340

ENSMUSG00000015396

ENSMUSG00000018339

ENSMUSG00000018927

ENSMUSG00000019122

ENSMUSG00000020099

ENSMUSG00000020437

ENSMUSG00000020607

ENSMUSG00000021388

ENSMUSG00000021457

ENSMUSG000000022102

ENSMUSG00000022488

ENSMUSG00000022901

ENSMUSG00000023046

ENSMUSG00000024679

ENSMUSG000000024965

ENSMUSG00000026109

ENSMUSG00000026177

ENSMUSG00000026395

ENSMUSG00000026399

ENSMUSG00000028581

ENSMUSG00000029771

ENSMUSG00000030787

ENSMUSG000000031827

ENSMUSG00000031906

ENSMUSG00000032332

ENSMUSG00000035095

ENSMUSG00000035208

ENSMUSG000000036887

ENSMUSG00000036905

ENSMUSG00000037379

ENSMUSG00000038147

ENSMUSG00000038642

ENSMUSG00000039109

ENSMUSG00000039476

ENSMUSG00000040552

ENSMUSG00000040612

ENSMUSG00000040950

ENSMUSG00000044337

ENSMUSG00000045573

ENSMUSG00000046743

ENSMUSG00000049130

ENSMUSG000000052384

ENSMUSG00000052688

ENSMUSG00000054203

ENSMUSG00000056069

ENSMUSG00000056938

ENSMUSG00000059412

ENSMUSG00000059498

ENSMUSG00000060586

ENSMUSG00000062593

ENSMUSG00000064372

ENSMUSG00000069763

ENSMUSG00000072596

ENSMUSG00000072620

ENSMUSG00000073421

ENSMUSG00000079018

ENSMUSG00000097233 readcount_amot/2 K0 aorta

158.927701543679

216.961569643465

1053.73474574084

173.756385209253

32.5248378550642

111.874554274348

239.844667429362

143.040000460753

78.5125901989447

2485.71158356384

707.295207631469

476.329244367314

345.887697572691

77.1633165981257

538.408993623667

1406.02622944515

213.973625481079

145.695459403768

205.41780877786

66.4110773964363

2884.24901370756

76.0534731344345

165.823698438286

428.493277023279

252.967326692869

120.375597657209

297.856093868171

465.732257990103

112.314140769318

542.979717509508

420.977724023766

164.547241376456

349.146794512607

44.906359424677

84.8499252503698

1888.43045766811

1436.86135647427

88.0293142776631

68.1694590534354

464.667808327248

1503.97106070614

249.429624873089

132.466267916088

229.441118259121

461.518760323747

1335.306800426

284.435378949316

1173.27680614689

193.925359200559

161.609513064848

311.450918170674

58.3391527588879

52.0027327519442

1667.9772179887

121.602644876039

404.525738192865

1199.0007722268

78.9260524892479

1173.17730974367

308.731091223443

37.6559320129037

133.548729536474

1230.3363026074

1618.26627631113

64.9621089152712 readcount wt aorta

59.7030557544915

100.502648381335

664.16622403341

82.7869817301975

69.2139660522236

15.1184535312192

52.6885310870578

57.6902339080253

29.0719859870467

1233.54481642737

170.544551968579

119.344035032349

496.115281245493

22.0118072197688

750.953249029995

673.183669052565

106.330862475272

51.5169124495549

98.2592233833908

29.7301164689561

1480.29003107507

33.0719080199057

90.238439882096

244.168057152686

135.598140854474

44.8203082114949

113.297994385314

238.530901942934

54.4093371094395

221.284214010384

199.139679950657

84.2907934668227

192.498035360874

4.66152356471479

29.273182687006

1033.03008726011

723.356029904941

9.28999489950067

23.4935738710137

211.512395936203

747.972796177522

141.757473194012

65.2414056829882

88.319984284168

205.384453243512

691.095361709203

164.418066334223

1606.07262123916

93.3120301481718

75.5828244406309

175.345615022865

21.2200107598094

21.5993828748247

2239.70157240921

36.8721972818891

205.145200130876

444.640925833898

28.3739988423478

724.65728653727

94.2885701350182

5.85817747873321

52.3537752797979

440.149774472324

772.609348595751

135.658897939805
log2FoldChange

$0.64533 \quad 0.00020619$

$0.6889 \quad 4.0862 \mathrm{e}-05$

$0.53107 \quad 0.00014112$

$0.67988 \quad 4.3562 \mathrm{e}-05$

$-0.651$

0.70298

0.79769

0.65278

0.70756

0.74646

0.93728

1.0472

$-0.44752$

0.77686

$-0.4257$

0.71181

0.66452

0.78403

0.64108

0.68252

0.61981

0.66918

0.65277

0.61223

0.6467

0.71883

0.98022

0.65498

0.71349

0.74918

0.70633

0.62867

0.6278

0.63442

0.78264

0.59504

0.62421

0.7633

0.65282

0.686

0.6244

0.63225

0.62538

0.69464

0.6718

0.68272

0.57468

$-0.41348$

0.64854

0.67969

0.59411

0.65648

0.69577

$-0.3859$

0.69779

0.62295

0.65852

0.75867

0.56873

0.81497

0.73401

0.77541

0.68531

0.65121

$-0.72186$

padj

0.047359

0.020194

0.040633

0.020478

0.040633

0.0091597

0.0065624

0.045604

0.020478

0.003354

0.00035054

$1.0458 \mathrm{e}-05$

0.046835

0.0091597

0.037515

0.0091597

0.020478

0.0091597

0.042328

0.022063

0.046835

0.034294

0.010434 
Term

neutrophil activation involved in immune response (GO:0002283)

neutrophil degranulation (GO:0043312)

neutrophil mediated immunity (GO:0002446)

inflammatory response (GO:0006954)

regulation of protein activation cascade (GO:2000257) regulation of complement activation (GO:0030449)

regulation of humoral immune response (GO:0002920)

regulation of immune effector process (GO:0002697)

regulation of acute inflammatory response (GO:0002673)

positive regulation of $T$ cell proliferation (GO:0042102)
Overlap

$12 / 483$

$11 / 479$

$11 / 487$

$7 / 252$

$5 / 108$

$5 / 109$

$5 / 113$

$5 / 114$

$5 / 121$

$4 / 61$
P-value

2.9981738632589026E-8

$2.655867971923774 E-7$

$3.131568369000276 \mathrm{E}-7$

$1.4068317157358681 E-5$

$2.291690762006761 \mathrm{E}-5$

$2.3960804936406545 \mathrm{E}-5$

$2.851149974163726 \mathrm{E}-5$

$2.9747522281288362 \mathrm{E}-5$

3.9607990367493474E-5

4.0770399717361916E-5
Adjusted P-value

1.7959061440920828E-5

$6.252698176770551 E-5$

$6.252698176770551 E-5$

0.0021067304943144624 0.002227345730811466

0.002227345730811466

0.002227345730811466

0.002227345730811466

0.0024421469430699785

0.0024421469430699785
Genes

PTPRC:SYK:SLC11A1;ITGB2;C5AR1;COTL1; C3AR1;CYBB;NCKAP1L;RETN;CTSS;CD55

PTPRC;SLC11A1;ITGB2;C5AR1;COTL1;C3AR1;CYBB; NCKAP1L;RETN;CTSS;CD55

PTPRC;SLC11A1;ITGB2;C5AR1;COTL1;C3AR1; CYBB;NCKAP1L;RETN;CTSS;CD55

NRROS;SYK;SLC11A1;ITGB2;C5AR1;C3AR1;CYBB C1QB;C1QA;C5AR1;C3AR1;CD55

C1QB;C1QA;C5AR1;C3AR1;CD55

C1QB;C1QA;C5AR1;C3AR1;CD55

C1QB;C1QA;C5AR1;C3AR1;CD55

C1QB;C1QA;C5AR1;C3AR1;CD55

CD86;PTPRC;SYK;NCKAP1L 


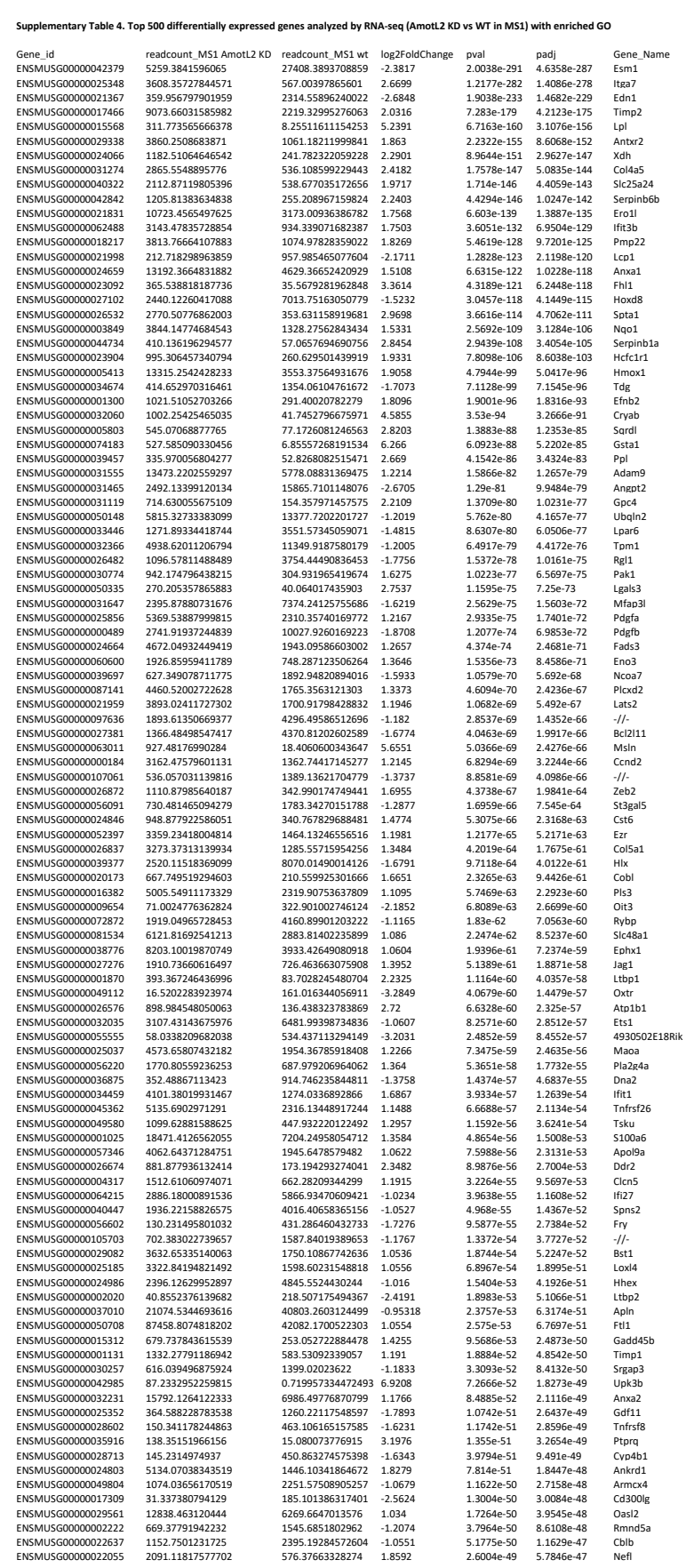

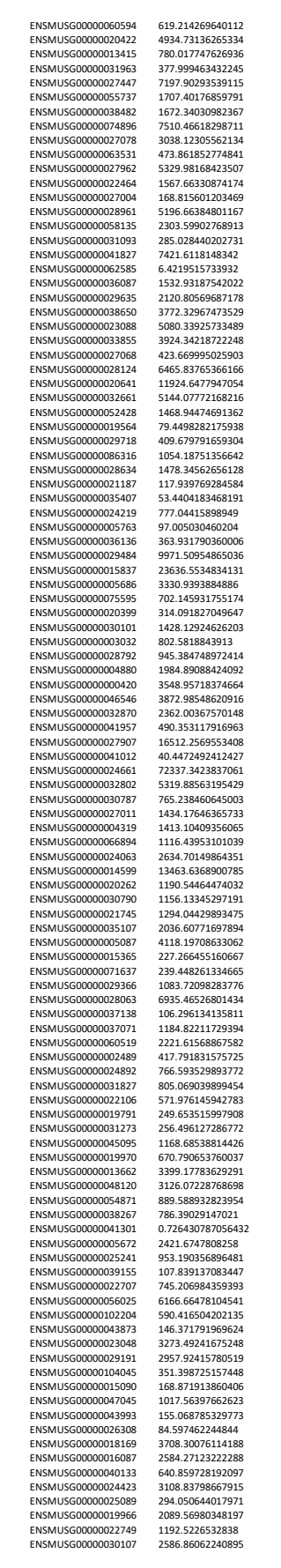

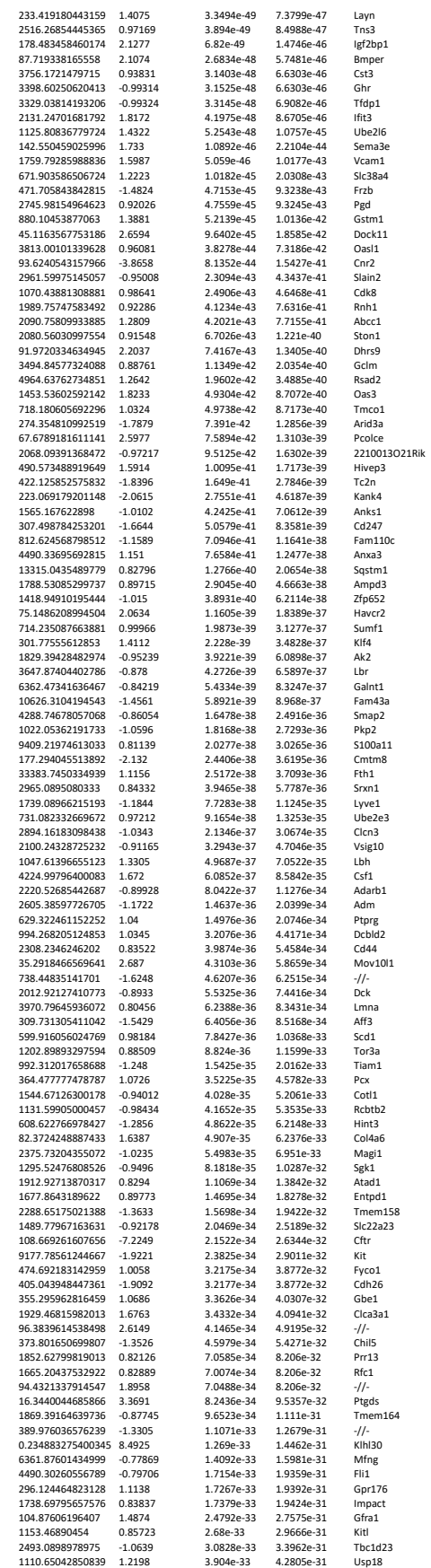

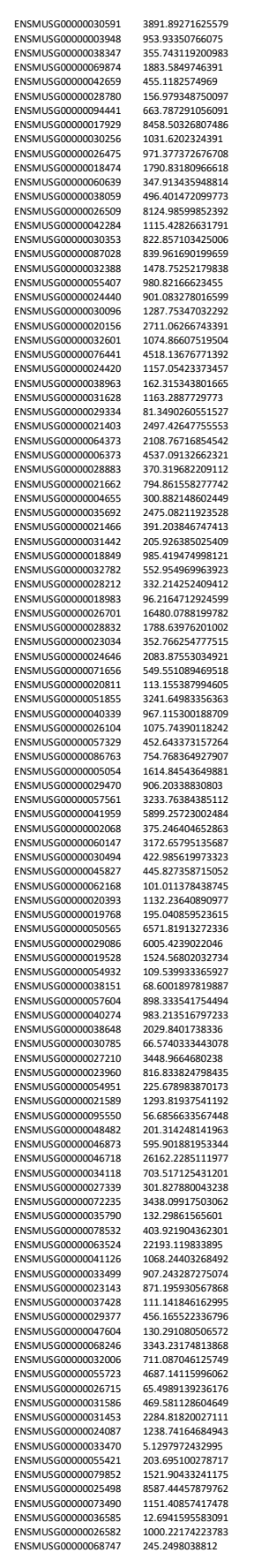

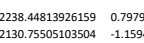

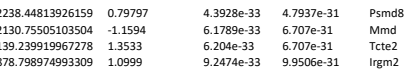

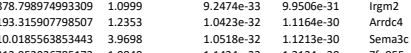

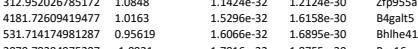

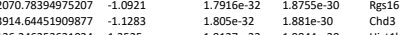

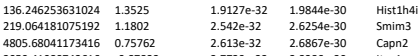

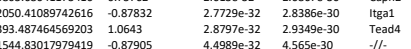

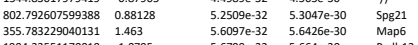

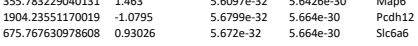

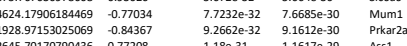

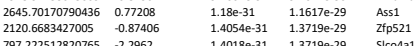

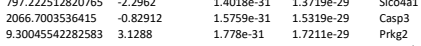

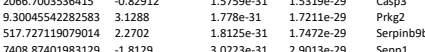

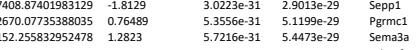

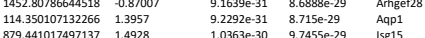

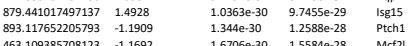

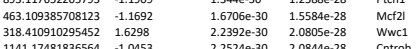

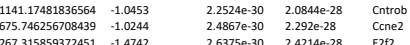

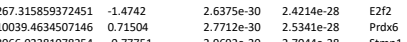

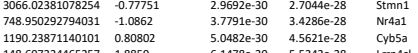

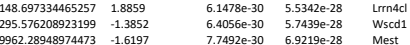

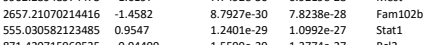

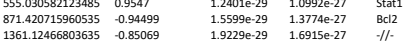

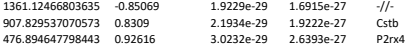

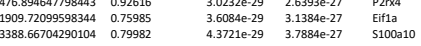

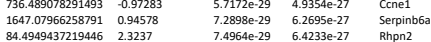

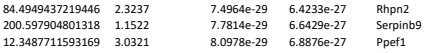

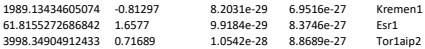

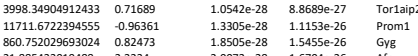

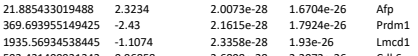

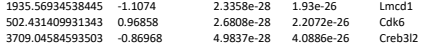

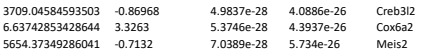

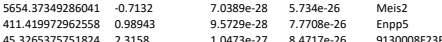

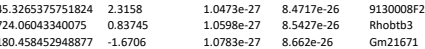

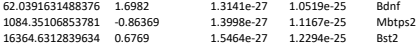

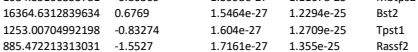

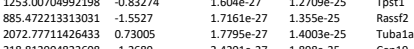

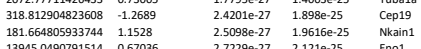

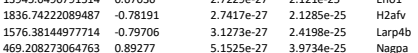

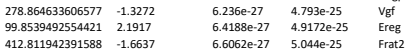

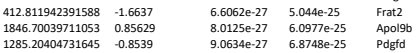

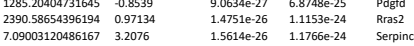

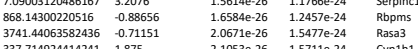

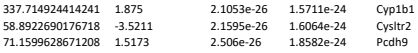

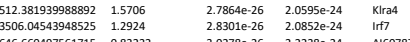

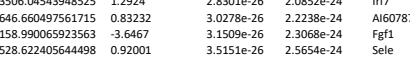




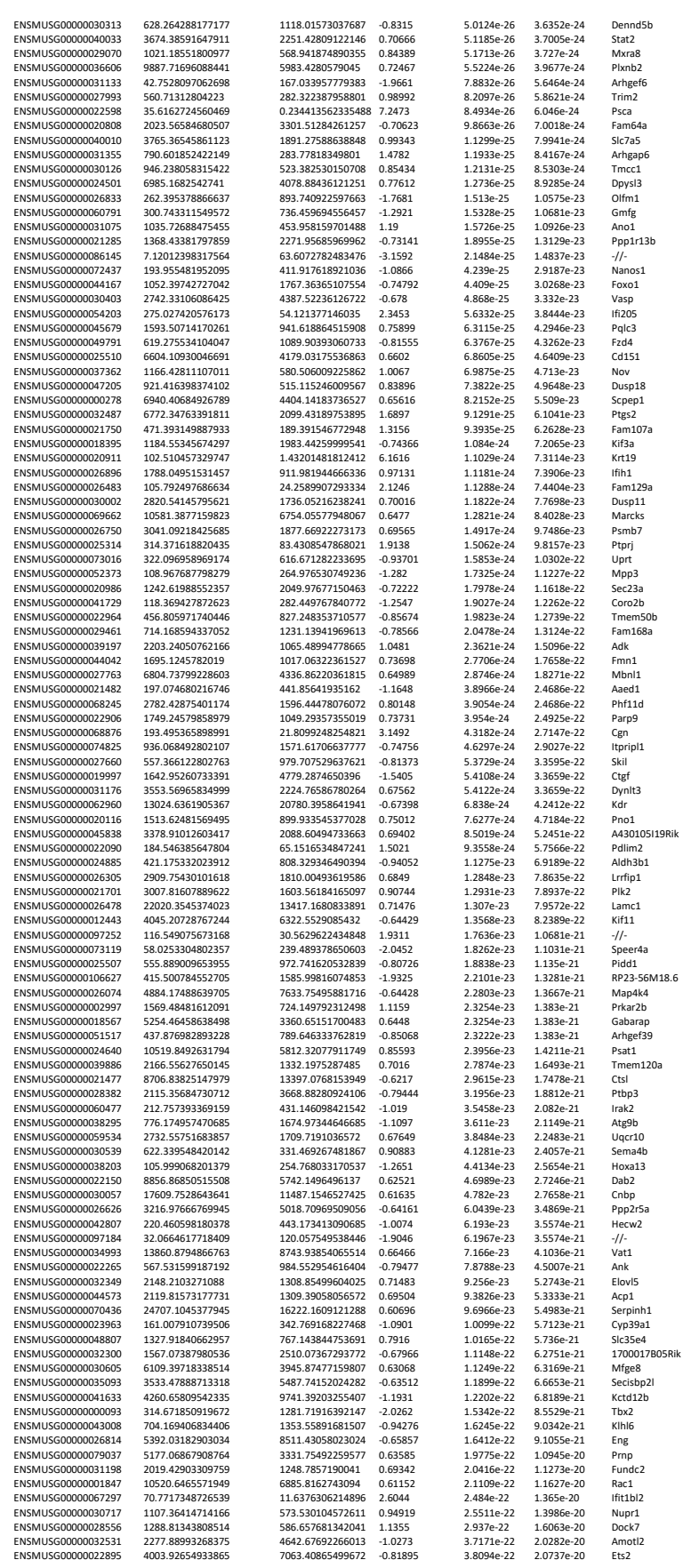

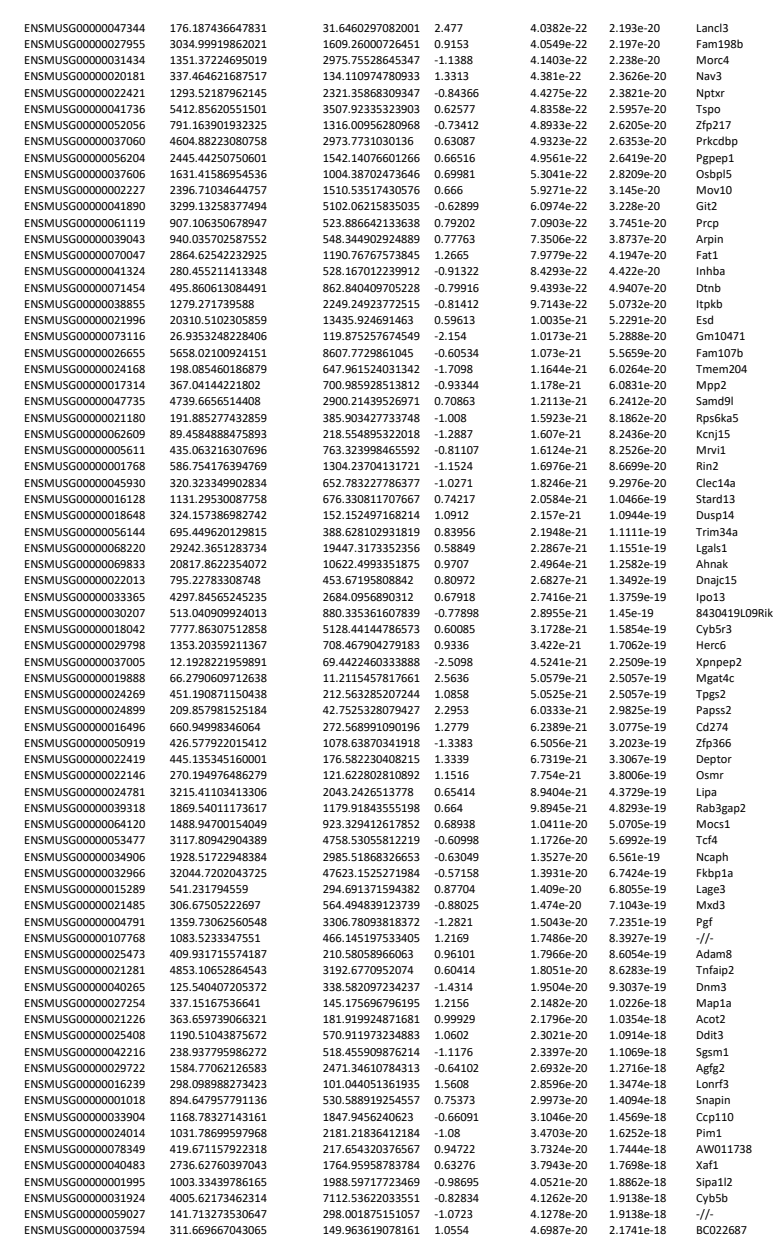


Term

regulation of cell migration (GO:0030334)

extracellular matrix organization (GO:0030198)

cellular response to type I interferon (GO:0071357) type I interferon signaling pathway (GO:0060337)

$$
\text { response to cytokine (GO:0034097) }
$$

negative regulation of cell migration (GO:0030336)

positive regulation of cell motility (GO:2000147)

positive regulation of fibroblast proliferation (GO:0048146)

positive regulation of cell proliferation (GO:0008284)

cytokine-mediated signaling pathway (GO:0019221)
Overlap

P-value

Adjusted P-value

$34 / 316$

$4.168055490873482 E-13$

$1.0586860946818643 \mathrm{E}-9$

$21 / 229$

$11 / 65$

$11 / 65$

$2.1678849334941394 E-7 \quad 2.753213865537557 E-4$

4.356552813789541E-7 $\quad 2.7664110367563584 \mathrm{E}-4$

$4.356552813789541 \mathrm{E}-7 \quad 2.7664110367563584 \mathrm{E}-4$

$15 / 138$

1.418875327663055E-6

6.344663285074403E-4

$14 / 121$

$1.498739358678993 \mathrm{E}-6$

$6.344663285074403 E-4$

$17 / 179$

$07 / 28$

$1.9113769513245516 \mathrm{E}-6$

$3.693471784353107 \mathrm{E}-6$

$27 / 424$

$5.994210083927416 \mathrm{E}-6$

0.001570063310885789

$35 / 633$

$6.1813516176605865 \mathrm{E}-6 \quad 0.001570063310885789$
Genes

CD151:CSF1-SEMA3C-SEMA3A-PDGFB-PDGFA-PTPRJ:SEMA3E;ADARB1; FGF1;PAK1;FAM110C;PDGFD;DPYSL3;LMNA;KDR;CYP1B1;RAC1;SRGAP3; ARPIN;EDN1;IAG1;ANXA1;ARHGEF39;SEMA4B;TPM1;BST2; TIAM1;DAB2;NAV3;PLXNB2;AMOTL2;SGK1;ENG

VCAM1;ITGA1;PDGFB;PDGFA;LOXL4;LAMC1;COL5A1;CTSL;KDR ; SERPINH1;TIMP2;CAPN2;COL4A6;CYP1B1;COL4A5; ITGA7;ADAM8;TIMP1;LCP1;CD44;DDR2

BST2;RSAD2;IFI27;STAT1;OAS3;STAT2;IRF7;ISG15;IFIT1;XAF1;IFIT3 BST2;RSAD2;III27;STAT1;OAS3;STAT2;IRF7;ISG15;IFIT1;XAF1;IFIT3

CD274;STAT1;ISG15;AFF3;OSMR;SELE;BST2;IRAK2;CASP3;DPYSL3;BCL2; TIMP2;ADAM9;TIMP1;XAF1

JAG1;TPM1;PTPRJ;ADARB1;PTPRG;BST2;NAV3;DPYSL3;CYP1B1;HMOX1; SRGAP3;TIMP1;ARPIN;ENG

EDN1;CD151;CSF1;SEMA3C;ARHGEF39;SEMA3A;SEMA4B;PDGFB;PDGFA; SEMAE;FGF1;TIAM1;DAB2;PAK1;FAM110C;PDGFD;KDR CDK6:PDGFB;S100A6;PDGFA;AQP1;EREG;DDR2

CSF1;PDGFB;PDGFA;LAMC1;FGF1;AQP1;EFNB2;ESM1;PAK1;CCND2; PDGFD;PIM1;KDR;HMOX1;TIMP1;EDN1;STAT1;CNBP;OSMR;PGF; TBX2;EREG;TIAM1;BST1;CDK6;S100A6;DDR2

CSF1;MAOA;PDGFB;PTGS2;IFIT1;FOXO1;IFIT3;PSMD8;PSMB7;RPS6KA5; IRAK2;CASP3;PIM1;HMOX1;TNFRSF8;TIMP1;ANXA1; VCAM1;ANXA2;RSAD2;STAT1;STAT2;ISG15;OSMR; EREG;BST2;IFI27;OAS3;KIT;BCL2;IRF7;LCP1;XAF1;SQSTM1;CD44 


\begin{tabular}{|c|c|c|c|c|}
\hline Antibody & Full name & Company & Catalog number & Dilution \\
\hline Isolectin B4 & Biotinylated griffonia simplicifolia lectin 1 & VECTOR & B-1205 & IF $1: 300$ \\
\hline GFP & Chk pAb to GFP & Abcam & ab13970 & IF $1: 200$ \\
\hline Phalloidin & Texas Red-X-Phalloidin & Life Technologies & T7471 & IF $1: 300$ for cell; $1: 200$ for retina \\
\hline Phalloidin & Phalloidin-Atto $647 \mathrm{~N}$ & Sigma & 65906-10nmol & IF 1:300 for cell; 1:200 for retina \\
\hline TO-PRO-3 & TO-PRO- iodide (642/661) & Life Technologies & T3605 & IF $1: 1000$ \\
\hline$\beta$-actin & Ms mAb to Actin & Abcam & ab3284 & WB-1:2000 \\
\hline VE-cadherin & Purified anti human CD144 (BV9) & Bio-legend & 348502 & IF 1:300 \\
\hline VE-cadherin & Purified rat anti mouse CD144 (11d4.1) & $\mathrm{BD}$ & 555289 & IF $1: 300$ \\
\hline VE-cadherin & Rabbit polyclonal VE Cadherin antibody & Abcam & 33168 & WB 1:500 \\
\hline Lamin A/C & Monocolonal Anti-Lamin A/C ab produced in mouse & Sigma & SAB4200236 & WB $1: 500$ \\
\hline Lamin B & $\mathrm{Rb} \mathrm{pAb}$ to LaminB1 & Abcam & ab16048 & WB 1:500 \\
\hline SUN2 & $\mathrm{Rb}$ mAb to SUN2 & Abcam & ab124916 & WB $1: 500$ \\
\hline GAPDH & Monocolonal Anti-GAPDH ab produced in mouse & Sigma & G8795 & WB $1: 3000$ \\
\hline CD31 (Pecam-1) & Purified rat anti mouse $\mathrm{CD} 31$ & $\mathrm{BD}$ & 553370 & IF $1: 300$ \\
\hline$\beta$-catenin & Purified mouse anti $\beta$-catenin & $\mathrm{BD}$ & 810154 & WB 1:500 \\
\hline CD45 & Mouse CD45 Antibody & R\&D systems & AF-114 & IF $1: 300$ \\
\hline AmotL2 & Angiomotin like 2 & Innovagen, Lund, Sweden & Purified from rabbit serum & WB-1:500, IP-1:1:200 (v/v) IF/IHC 1:100 \\
\hline
\end{tabular}




\section{Secondary Antibody}

$\mathrm{ECL}^{\circledR}$ Anti-rabbit IgG

For WB

$\mathrm{ECL}^{\circledR}$ Anti-mouse IgG

$\mathrm{ECL}^{\circledR}$ Anti-rat IgG

goat anti-Mouse $\lg G(\mathrm{H}+\mathrm{L})$

goat anti-Rat IgG $(\mathrm{H}+\mathrm{L})$

donkey anti-Sheep IgG $(\mathrm{H}+\mathrm{L})$

goat anti-Chicken IgG $(\mathrm{H}+\mathrm{L})$

donkey anti-Goat IgG $(\mathrm{H}+\mathrm{L})$

chicken anti-Rabbit IgG $(\mathrm{H}+\mathrm{L})$

donkey anti-Mouse Ig $\mathrm{G}(\mathrm{H}+\mathrm{L})$

donkey anti-Rabbit IgG $(\mathrm{H}+\mathrm{L})$

For IF

staining

goat anti-Mouse IgG $(\mathrm{H}+\mathrm{L})$ goat anti-Rat IgG $(\mathrm{H}+\mathrm{L})$

goat anti-Rabbit IgG $(\mathrm{H}+\mathrm{L})$

goat anti-Rat Ig $(\mathrm{H}+\mathrm{L})$

donkey anti-Mouse $\lg \mathrm{G}(\mathrm{H}+\mathrm{L})$

donkey anti-Rabbit IgG $(\mathrm{H}+\mathrm{L})$

goat anti-Mouse IgG $(\mathrm{H}+\mathrm{L})$

goat anti-Rabbit IgG $(\mathrm{H}+\mathrm{L})$

Streptavidin

Streptavidin

\section{Conjugation}

HRP linked whole antibody from donkey

HRP linked whole antibody from donkey

HRP linked whole antibody from donkey

Alexa Fluor ${ }^{\circledR} 405$ conjugate

Alexa Fluor ${ }^{\circledR} 488$ conjugate

Alexa Fluor ${ }^{\circledR} 488$ conjugate

Alexa Fluor ${ }^{\circledR} 488$ conjugate

Alexa Fluor ${ }^{\circledR} 488$ conjugate

Alexa Fluor ${ }^{\circledR} 488$ conjugate

Alexa Fluor ${ }^{\circledR} 555$ conjugate

Alexa Fluor ${ }^{\circledR} 555$ conjugate

Alexa Fluor ${ }^{\circledR} 594$ conjugate

Alexa Fluor ${ }^{\circledR} 594$ conjugate

Alexa Fluor ${ }^{\circledR} 594$ conjugate

Alexa Fluor ${ }^{\circledR} 633$ conjugate

Alexa Fluor ${ }^{\circledR} 647$ conjugate

Alexa Fluor ${ }^{\circledR} 647$ conjugate

$\mathrm{Cy}^{\circledR}{ }^{\circledR}$

Cy3

Alexa Fluor ${ }^{\circledR} 405$ conjugate

Alexa Fluor ${ }^{\circledR} 488$ conjugate

\section{Catalog number}

NA934V

NA931V

NA935V

A31553

A11006

A11015

A11039

A11055

A21441

A31570

A31572

A11005

A11007

A11037

A21094

A31571

A31573

A10521

A10520

S32351

S32354

\section{Company}

GE Healthcare

GE Healthcare

GE Healthcare

LifeTechnologies

LifeTechnologies

LifeTechnologies

LifeTechnologies

LifeTechnologies

LifeTechnologies

LifeTechnologies

LifeTechnologies

LifeTechnologies

LifeTechnologies

LifeTechnologies

LifeTechnologies

LifeTechnologies

LifeTechnologies

LifeTechnologies

LifeTechnologies

LifeTechnologies

LifeTechnologies
Dilution

1:10000

$1: 10000$

$1: 10000$

$1: 500$

$1 \cdot 500$

1:500

1.500

$1: 500$

$1 \cdot 500$

$1: 500$

$1 \cdot 500$

$1: 500$

$1 \cdot 500$

$1: 500$

$1: 500$

1:500

1.500

1:500

$1: 500$

$1: 500$

$1: 500$ 
Reagents

$\begin{array}{lc}\text { In vivo } & \text { Reagent } \\ \text { In vitro } & \text { Tamoxifen } \\ & \text { Blebbistatin } \\ \text { Cytoclaxin D } \\ \text { Paraformaldehyde solution 4\% in PBS } \\ \text { Fluoroshield with DAPI } \\ \text { Western Lightning Plus-ECL } \\ \text { Protein G Sepharose 4 fast flow } \\ \text { IgG from rabbit serum } \\ \text { IgG from mouse serum } \\ \text { Polybrene (Hexadimethrine Bromide) } \\ \text { Polybrene (Hexadimethrine Bromide) }\end{array}$

\section{Company}

Sigma

Sigma

Sigma

ChemCruz

Sigma

PerkinElmer

GE Healthcare

Sigma

Sigma

VectorBuilder

Sigma

\section{Catalog number}

T5648

B0560

C8273

sc-281692

F6057

203-170071

17-0618-01

18140

18765

provided with lentivirus

H9268-5G 
Lenti-virus

Name

Non-Targeting (scrambled) shRNA Control

human AmotL2 shRNA virus
Company

Sigma

Sigma
Sequence if it's customized product

5'-GCGAGAGAAGGAGGAGCAGATC-3' 
TaqMan Probes

\begin{tabular}{|l|l|}
\hline Company & Thermo-Fisher \\
\hline Species & Mouse \\
\hline Gene & Taqman reference \\
\hline TNF & Mm00443258_m1 \\
\hline CD68 & Mm03047343_m1 \\
\hline Cxcl10 & Mm00445235_m1 \\
\hline Icam1 & Mm00516023_m1 \\
\hline Vcam1 & Mm01320970_m1 \\
\hline Cd8a & Mm01188922_m1 \\
\hline Cd4 & Mm01185100_m1 \\
\hline Ccl2 & Mm00441242_m1 \\
\hline Ccl5 & Mm01302427_m1 \\
\hline Cd19 & Mm00515420_m1 \\
\hline IL6 & Mm00446190_m1 \\
\hline HPRT & Mm03024075_m1 \\
\hline
\end{tabular}

